\title{
Recent Advances in Ambipolar Transistors for Functional Applications
}

\author{
Yi Ren, Xiaoyang Yang, Li Zhou, Jing-Yu Mao, Su-Ting Han,* and Ye Zhou*
}

\begin{abstract}
Ambipolar transistors represent a class of transistors where positive (holes) and negative (electrons) charge carriers both can transport concurrently within the semiconducting channel. The basic switching states of ambipolar transistors are comprised of common off-state and separated on-state mainly impelled by holes or electrons. During the past years, diverse materials are synthesized and utilized for implementing ambipolar charge transport and their further emerging applications comprising ambipolar memory, synaptic, logic, and light-emitting transistors on account of their special bidirectional carrier-transporting characteristic. Within this review, recent developments of ambipolar transistor field involving fundamental principles, interface modifications, selected semiconducting material systems, device structures, ambipolar characteristics, and promising applications are highlighted. The existed challenges and prospective for researching ambipolar transistors in electronics and optoelectronics are also discussed. It is expected that the review and outlook are well timed and instrumental for the rapid progress of academic sector of ambipolar transistors in lighting, display, memory, as well as neuromorphic computing for artificial intelligence.
\end{abstract}

\section{Introduction}

With the rapid advances of network and information techniques, the emerging artificial intelligence (AI) which refers to the intelligence exhibited by an artificially manufactured electronic system and the Internet of things (IoT) which connects common physical objects with cyber space systems both have provoked multitudes of focus in industrial and academic

Y. Ren, J.-Y. Mao, Prof. Y. Zhou

Institute for Advanced Study

Shenzhen University

Shenzhen 518060, P. R. China

E-mail: yezhou@szu.edu.cn

Dr. X. Yang, Dr. L. Zhou, Prof. S.-T. Han

Institute of Microscale Optoelectronics and Key Laboratory

of Optoelectronic Devices and Systems of Ministry of Education

and Guangdong Province

College of Optoelectronic Engineering

Shenzhen University

Shenzhen 518060, P. R. China

E-mail: sutinghan@szu.edu.cn

Prof. S.-T. Han

Department of Electrical Engineering and Computer Science

The University of Michigan

Ann Arbor, MI 48109, USA

The ORCID identification number(s) for the author(s) of this article can be found under https://doi.org/10.1002/adfm.201902105.

DOI: $10.1002 / \mathrm{adfm} .201902105$ communities. For the sake of the successful implementation of their intricate functionalities, diverse fundamental electronic constituents are imperative to act as functional system blocks. As one of the most significant components, transistors are the foundation and key ingredients of modern electronic devices and products. ${ }^{[1]}$ Since the 1950s, transistors have gradually replaced vacuum tubes and finally realized mass production of integrated circuits and microprocessors. Transistors possessing advantages of low cost, flexibility and reliability have revolutionized electronics, making electronic devices smaller, cheaper, and much more efficient. ${ }^{[2-9]}$

In accordance with switching characteristic and predominant charge carriers within semiconductors, the polarity of transistors can be categorized as unipolar (hole-dominated p-type or electron-dominated n-type) or ambipolar (comparable hole and electron contributions). Apparently, ambipolar transistors are capable of integrating p- and n-type electrical performance into single device and thereby have aroused plenty of concern and discussion of researchers from various fields such as organic chemistry and device science. ${ }^{[10]}$ Nonetheless, up to now vast majority of transistors display unipolar dominated charge transport in spite of the theory that each semiconductor is capable of migrating holes and electrons according to semiconductor physics. For purpose of fabricating high-performance ambipolar transistors with large carrier mobility and high on/off ratio as well as low threshold voltage, appropriate semiconducting materials with relatively small bandgap, smooth and flawless interface characteristics together with electrode contacts with eligible charge injection barrier are both pivotal. During past years, miscellaneous semiconducting materials comprising organic small molecules, ${ }^{[11]}$ conjugated polymers, ${ }^{[12]}$ 2D materials, ${ }^{[13]}$ and organic-inorganic hybrid materials ${ }^{[14]}$ have been utilized for realizing ambipolar charge transport and promising functional applications. Ambipolar transistors which transport both types of charge carriers synchronously make the fabrication of complementary metal-oxide-semiconductor (CMOS) inverters simpler due to controllable separated unipolar mode of them, meanwhile the manufacture of ambipolar light-emitting transistors which integrate switching characteristic (property of transistors) and light emission capacity (nature of light-emitting diodes) into an unitary device will be more efficient. ${ }^{[10,15]}$ Moreover, by adding another two layers (floating gate and tunneling dielectric), 
ambipolar transistors can be utilized as well-known flash memory that can exhibit much larger memory window due to bidirectional threshold voltage shifts on the basis of trapping/ detrapping mechanism. ${ }^{[16]}$ Inspired by the similarity between the fluxion of neurotransmitters in synaptic cleft and the actional transport and trapping operation of charge carriers, ambipolar transistors also possess great potential for artificial synaptic emulation and hence neuromorphic computing in artificial intelligence. ${ }^{[17]}$

The overview structure of this review is described as follows. The basic structure, energy band alignment and fundamental principles of ambipolar transistors are first introduced. Particularly, functionalities and influences of semiconducting materials, interface characteristics and electrode contacts on ambipolar performance are elucidated. Then, the recent selected diverse semiconducting materials for instance organic and 2D materials as well as various device architectures of ambipolar transistors are contrasted and the merits and demerits of them are also analyzed. Within stated various material systems, in spite of existed challenges, basic mechanisms of fabricated transistors are well comprehended and hence some realistic applications begin to appear. Thus, the promising applications of ambipolar transistors comprising ambipolar flash memories, artificial synaptic transistors, logic devices and light-emitting transistors are then focused (Figure 1). Finally, the existing challenges and outlook of the future developments and applications of ambipolar transistors in printed electronics are discussed. Hopefully, this review can provide instructive roadmaps for the evolution of ambipolar transistor area.

\section{Structures and Fundamental Principles of Ambipolar Transistors}

Ambipolar transistors represent transistors that allow synchronous transport of electrons and holes and their accumulation within semiconductors. ${ }^{[18,19]}$ An ambipolar transistor is usually constructed from gate electrode, dielectric, semiconducting layer, and two source-drain electrodes (Figure 2a). The first three parts make up a metal-insulator-semiconductor (MIS) architecture which is capable of propelling holes or electrons to accumulate at the interface between dielectric and semiconducting layer via exerting a gate bias with specific voltage polarity. The additional source-drain electrodes coming into contact with the interface of active layer dominate the injection of charge carriers which are necessary for the subsequent accumulation process. The injected holes and electrons can transfer in the light of various biases applied at these three terminals. Thus, both the amount and polarity of charge carriers within accumulation zone are different under gate biases with diverse amplitudes and polarities (positive gate bias for electron accumulation whereas negative one for hole accumulation), which finally leads to differences in the conductivity of the transistors (Figure $2 b, c)$.

In terms of band bending of MIS junction (Figure 2d), each transistor is capable of accumulating two types of charge carriers (lie with the external electrical bias on gate electrode) in the ideal state according to electric field effect. Nonetheless, vast majority of transistors display unipolar electrical performance (only p- or n-type). In fact, subsistent charge traps such

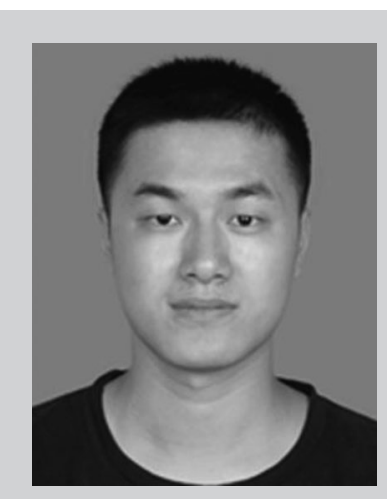

Yi Ren received his physics bachelor's degree from Institute for Advanced Study at Shenzhen University, Guangdong, China, in 2018. $\mathrm{He}$ is now a postgraduate student at the Institute for Advanced Study in Shenzhen University. His research interests include organic/inorganic materials for nanoscale devices, such as data storage and artificial synapse devices.

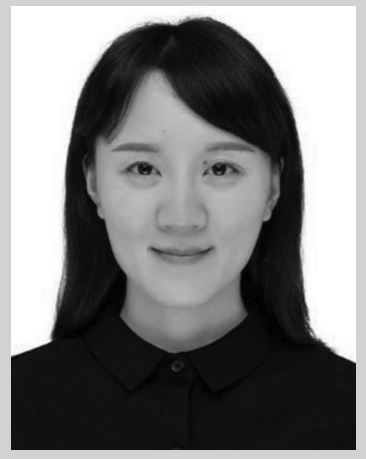

Su-Ting Han is an associate professor at Shenzhen University and a visiting associate professor at The University of Michigan. She received her M.Sc. degree in analytical chemistry from Hong Kong Baptist University and her Ph.D. degree in physics and materials science from City University of Hong Kong. Her research interests include functional electronic devices and flexible, stretchable, and wearable electronics.

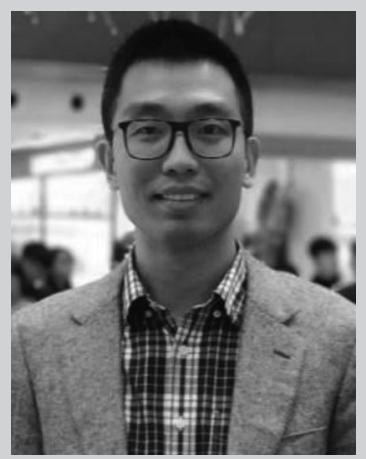

Ye Zhou is an IAS Fellow and group leader in the Institute for Advanced Study, Shenzhen University. His research interests include flexible and printed electronics, organic/inorganic semiconductors, surface and interface physics, nanostructured materials, and nanoscale devices for technological applications, such as logic circuits, data storage, energy harvesting, photonics, and sensors.

as defects and vacancies within semiconducting layer and the interface between dielectric and semiconducting layers can capture minority carriers and hence impede the accumulation of them. Besides, various bandwidths between conduction and valance band induce different transport capabilities of those two charge carriers and hence asymmetric accumulation abilities of them. These reasons both make the accumulation of minority carriers challenging in common transistors. Researchers name this condition strong inversion condition and have discovered several methods to solve these difficulties and achieved ambipolar transport. ${ }^{[20,21]}$ 


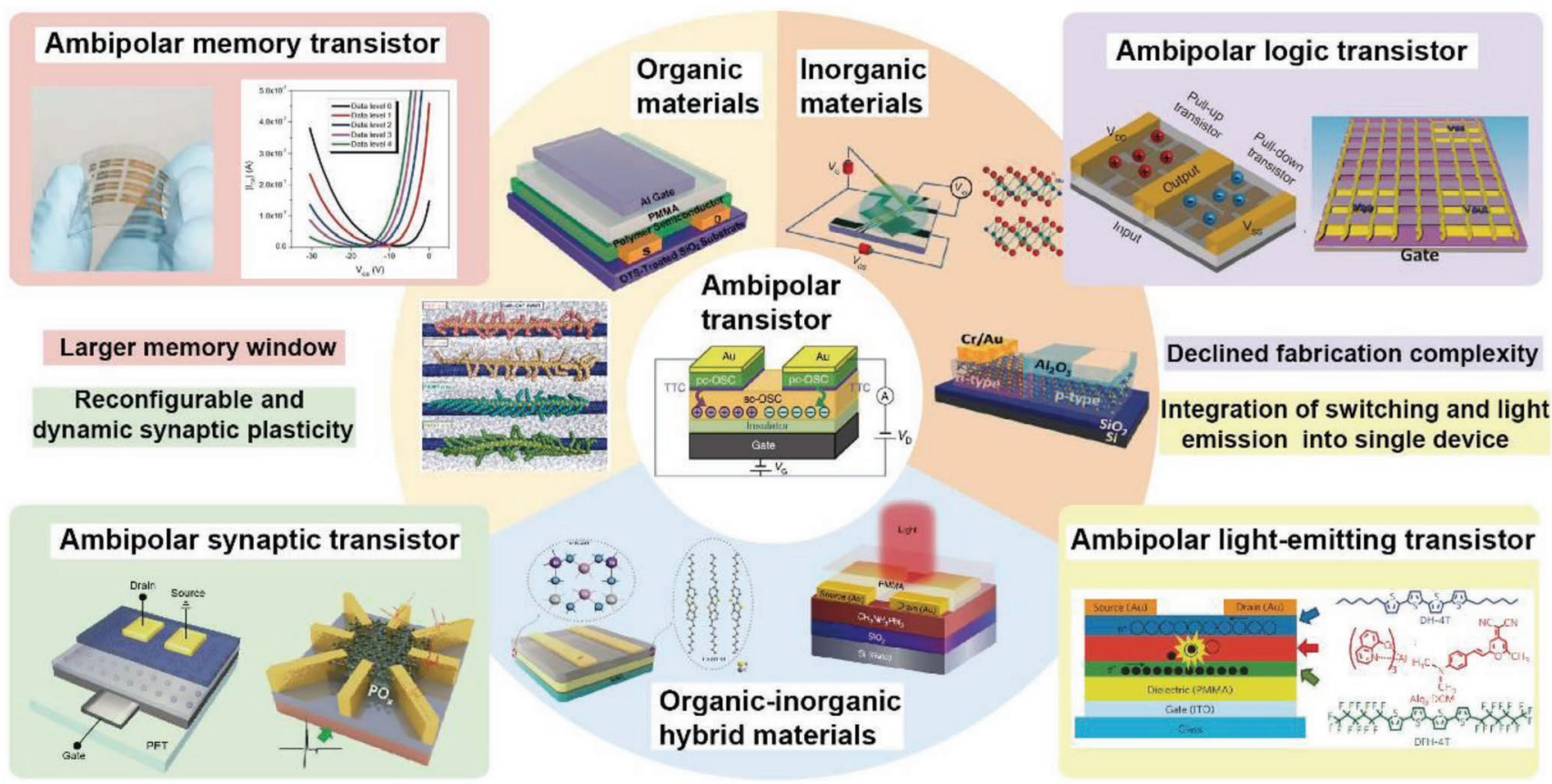

Figure 1. Schematic diagram of ambipolar transistors on the basis of diverse materials and their promising applications. $[14,17,25,121,260,284,302,397,417,450$, 455,465,518] Ambipolar transistor. Reproduced with permission. ${ }^{[121]}$ Copyright 2017, Nature Publishing Group. Organic materials. Reproduced with permission. ${ }^{[25]}$ Copyright 2017, American Chemical Society (top). Reproduced with permission. ${ }^{[260]}$ Copyright 2014, American Chemical Society (bottom). Inorganic materials. Reproduced with permission. ${ }^{[302]}$ Copyright 2012, American Chemical Society (top). Reproduced with permission. ${ }^{[284]}$ Copyright 2017, Wiley-VCH (bottom). Organic-inorganic hybrid materials. Reproduced with permission. ${ }^{[397]}$ Copyright 2018, Wiley-VCH (left). Reproduced with permission. ${ }^{[14]}$ Copyright 2015, Nature Publishing Group (right). Ambipolar memory transistor. Reproduced with permission. ${ }^{[417]}$ Copyright 2013, Nature Publishing Group. Ambipolar synaptic transistor. Reproduced with permission. ${ }^{[450]}$ Copyright 2018, Wiley-VCH (left). Reproduced with permission. ${ }^{[17]}$ Copyright 2016, Wiley-VCH (right). Ambipolar logic transistor. Reproduced with permission. ${ }^{[455]}$ Copyright 2015, Nature Publishing Group (left). Reproduced with permission. ${ }^{[465]}$ Copyright 2016, American Chemical Society (right). Ambipolar light-emitting transistor. Reproduced with permission. ${ }^{[518]}$ Copyright 2010, Nature Publishing Group.

Despite different amounts of electrons and holes exit within semiconductors, both electrons and holes make a contribution to the entire drain current within channel when the transistor is operated in ambipolar mode. To date, scientists have put forward several theories to explain the operating principles of ambipolar transistors. Majority of them are on the basis of Pao-Sah description (mainly utilized in unipolar transistors ${ }^{[22]}$ and graduate channel approximation. Nonetheless, these models ignore the recombination operation of charge carriers and contacts characteristics, which both are crucial processes for ambipolar performance. Subsequently, modified models were brought up individually by Paasch et al. and Schmechel et al., respectively. Paasch et al. took contacts and recombination into consideration and utilized 2D numerical simulations for single-layer model architecture, which successfully comprehended previous bilayer ambipolar transistors. ${ }^{[23]}$ Meanwhile, Schmechel et al. proposed equivalent-circuit model which was composed of resistor-capacitor interwoven networks and obtained Shockley-like equations based on an assumption (large recombination probability of charge carriers), which finally resulted in good matching with the experimental results. ${ }^{[24]}$ With regard to ambipolar transistors, both two types of charge carries can be accumulated simultaneously and thereby two various threshold biases ( $V_{\text {th,n }}$ for electrons while $V_{\text {th,p }}$ for holes) are needed. On the basis of aforementioned assumption, obtained three various operation schemes (operated at electron accumulation regime) are listed as follows:
Unipolar state: The required range of drain-source voltage is $V_{\mathrm{d}} \leq\left(V_{\mathrm{g}}-V_{\text {th,n }}\right)$

$\left|I_{\mathrm{d}}\right|=\frac{W C}{L} \mu_{\mathrm{n}}\left[\left(V_{\mathrm{g}}-V_{\mathrm{th}, \mathrm{n}}\right)-\frac{1}{2} V_{\mathrm{d}}\right] V_{\mathrm{d}}$

Saturation state: The required scope of drain-source bias is $V_{\mathrm{d}} \leq\left(V_{\mathrm{g}}-V_{\mathrm{th}, \mathrm{p}}\right)$ but $V_{\mathrm{d}} \geq\left(V_{\mathrm{g}}-V_{\mathrm{th}, \mathrm{n}}\right)$ (meaningless when $V_{\text {th,p }}=V_{\text {th,n }}$ )

$\left|I_{\mathrm{d}}\right|=\frac{W C_{1}}{2 L} \mu_{\mathrm{n}}\left(V_{\mathrm{g}}-V_{\text {th }, \mathrm{n}}\right)^{2} H\left(V_{\mathrm{g}}-V_{\text {th }, \mathrm{n}}\right)$

Ambipolar state: The required range of $V_{\mathrm{d}}$ is $V_{\mathrm{d}} \geq\left(V_{\mathrm{g}}-V_{\mathrm{th}, \mathrm{p}}\right)$

$\left|I_{\mathrm{d}}\right|=\frac{W C_{1}}{2 L}\left\{\mu_{\mathrm{n}}\left(V_{\mathrm{g}}-V_{\text {th }, \mathrm{n}}\right)^{2} H\left(V_{\mathrm{g}}-V_{\text {th }, \mathrm{n}}\right)+\mu_{\mathrm{p}}\left[V_{\mathrm{d}}-\left(V_{\mathrm{g}}-V_{\text {th }, \mathrm{p}}\right)\right]^{2}\right\}$

where $V_{\mathrm{d}}, V_{\mathrm{g}}, I_{\mathrm{d}}, \mathrm{W}, L, C, C_{1}, \mu_{\mathrm{n}}, \mu_{\mathrm{p}}$, and $H(\mathrm{x})$ represent sourcedrain voltage, gate voltage, source-drain current, channel width, channel length, capacitances per unit area, electron mobility, hole mobility, as well as Heaviside-step function, respectively.

According to the constitution of semiconductors, the architectures of most ambipolar transistors can be divided into three categories: single component, blends and bilayer (Figure 2e). First, Single-component organic materials with appropriate energy levels and interface contacts and 2D materials with intrinsic ambipolar charge transport characteristics can be utilized individually for ambipolar behavior and just only one 
(a)

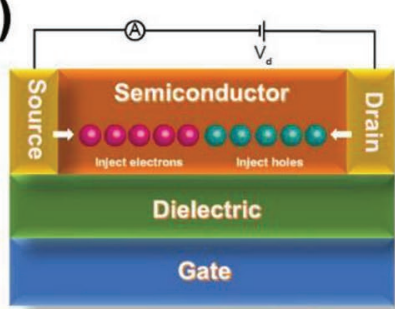

(d)

No gate bias $(\mathbf{V g}=\mathbf{0})$

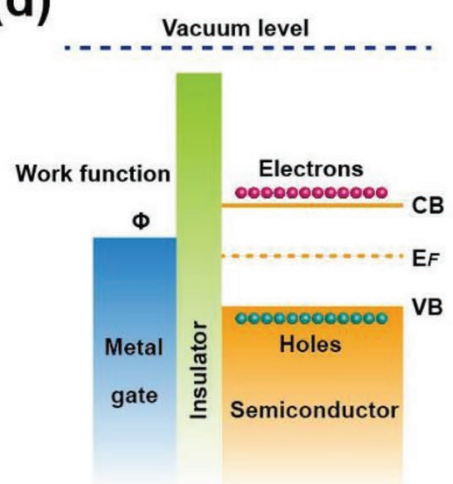

(e)

Initial state $(\mathbf{V g}=\mathbf{0})$

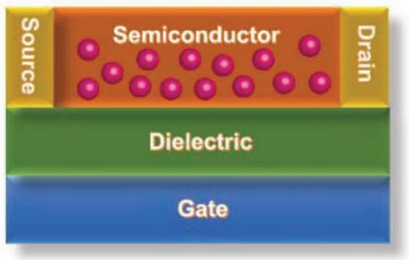

Single-component (b)

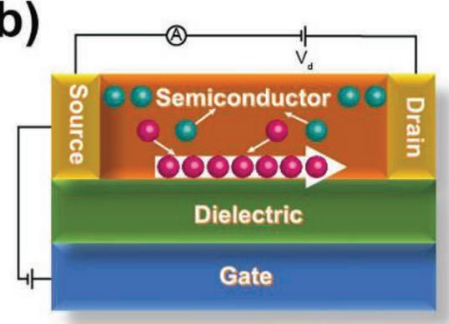

Electron enhancement $(\mathbf{V g}>\mathbf{0})$

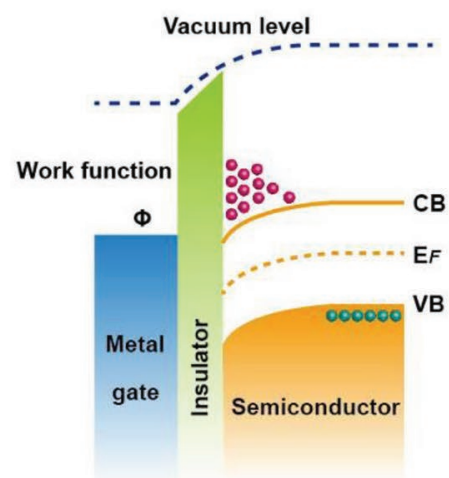

Electron accumulation $(\mathrm{Vg}>0)$

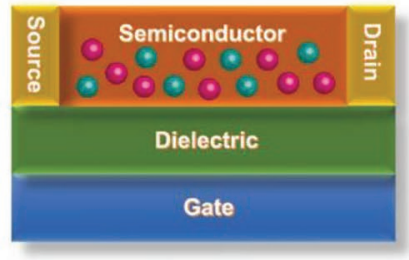

Blends

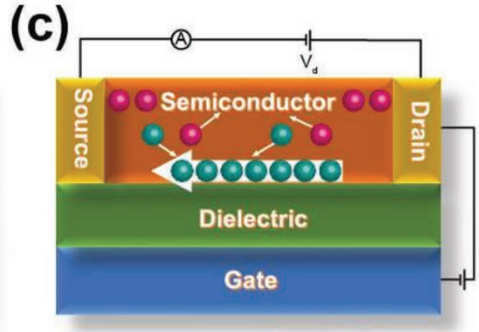

Hole enhancement $(\mathrm{Vg}<0)$

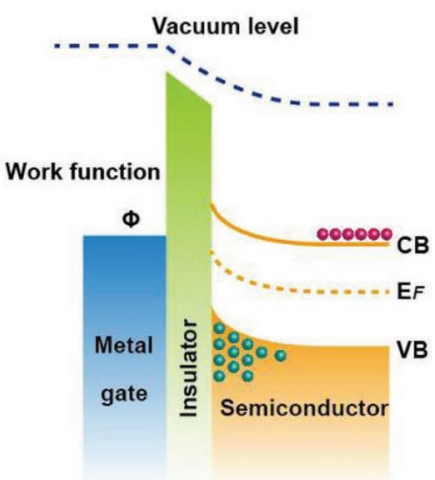

Hole accumulation $(\mathrm{Vg}<0)$

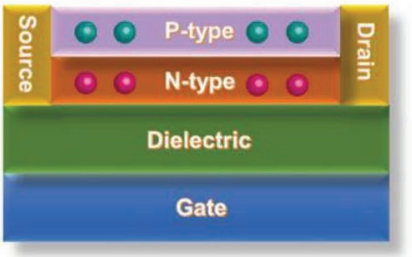

Bilayer

Figure 2. a-c) Schematic configurations of ambipolar transistors and their working mechanisms under constant source-drain bias and various gate biases (a: no gate bias; b: positive gate bias; c: negative gate bias). d) Energy band alignments of MIS structure under zero (left), positive (middle) and negative (right) gate voltages, respectively. e) The most common utilized architectures of ambipolar transistors including single component (left), blends (middle) and bilayer (right), respectively. The violet and cyan balls represent electrons and holes in (a)-(d) and refer to two various semiconducting materials in (e).

material is responsible for the electron and hole transport and accumulation within semiconducting channel. ${ }^{[13,25]}$ Second, thin films of semiconducting polymer blends, mixtures and composites fabricated via solution-processing and vacuum coevaporation can also achieve ambipolar performance and controlling the morphology of blends well is extremely crucial for realizing better ambipolar behavior. ${ }^{[26]}$ Apparently, these two or three materials (p- or n-type) take charge of electron and hole transport separately and efficiently but also in identical and entire blending semiconductors. Third, bilayer configuration (van der Waals heterostructures for 2D materials) usually consists of two stacked $\mathrm{p}$ - and $\mathrm{n}$-type semiconducting layers for respective hole and electron transfer processes (two detached semiconducting channels) and deposition order, relative film thickness and in particular $\mathrm{p} / \mathrm{n}$ interface are pivotal for wellbalanced transport of both two types of charge carriers. ${ }^{[27]}$ In the following main text, ambipolar transistors are categorized based on organic, inorganic and organic-inorganic hybrid materials, which is still consistent with the classification on the basis of the architectures of ambipolar transistors because all three structures of ambipolar transistors are contained and embodied in each material system (organic, inorganic, or organic-inorganic).

Semiconducting materials, interface characteristics together with electrode contacts are three extremely crucial aspects which require considerable designs for effective ambipolar behavior. The selected semiconductors should possess appropriate energy band alignments with favorable and comparable conduction capabilities of both two types of charge carriers and meanwhile suitable bandgap or potential barrier (e.g., smaller energy gap and thereby lower barrier for injecting electrons and holes in semiconductor materials) are beneficial for the transport of electrons and holes. Besides, the smooth and flawless interface between semiconducting and insulating layers contributes to unimpeded and fluent drift of holes and electrons and hence well-balanced ambipolar performance. 
In addition to these two factors, semiconductor/metal interface dominating the vital charge injection process from sourcedrain electrodes to semiconducting channel is determinant in ambipolar operation. The charge injection efficiency is determined by the concept of contact resistance which depends on the separated hole/electron injection barriers extracted from the energy band structures between the work function of electrodes and valence band/conduction band of the inorganic materials or highest occupied molecular orbital (HOMO)/lowest unoccupied molecular orbital (LUMO) of the organic materials. ${ }^{\text {[28] }}$ Scientists discovered that the Fermi level arrangements of semiconducting materials and source-drain electrodes were unable to happen efficiently and they proposed several factors to adjust this Mott-Schottky-type band bending within the interface between semiconducting layer and source-drain electrodes. For example, one is image-charge effect (provenience of the inorganic semiconducting band bending) which is capable of reducing the energy gaps of organic semiconducting materials and hence lowing the Schottky barrier for charge injection. ${ }^{28,29]}$ Another one is autogeneous formation of electric dipole layer at the interface between semiconductor and electrodes that can induce the movement of vacuum level and thereby generate various barriers. ${ }^{[30]}$ Thus, apparently, the authentic Schottky barrier is unrestricted and fluctuant. It is also worth mentioning that favorable Ohmic contact and low contact resistance at source-drain electrodes/semiconducting materials interface is advantageous to tackle the detrimental problems such as irregular electrical curves and majority of voltage drop occupied by contacts induced by non-Ohmic contacts. Therefore, selection of suitable electrode and semiconductor materials or modifying the interface of source-drain electrodes with selfassembled monolayers both give rise to better Ohmic contacts and thereby superior ambipolar behavior.

\section{Ambipolar Organic Semiconducting Materials}

As mentioned above, balanced energy band alignments are indispensable for favorable ambipolar behaviors. The HOMO level is responsible for holes injection and the LUMO level is in charge of electrons injection of the organic semiconductor, and they should match well with the work function of the source/ drain electrodes. However, significant challenges still exist as only single kind of charge carriers (holes or electrons) can be injected and transported for given metal electrodes on account of relatively wide bandgap $(2-3 \mathrm{eV})$ of mostly reported organic semiconductors. In this respect, the materials of the active layer with comparatively narrow bandgap $(1-2 \mathrm{eV})$ have been utilized to reduce the charge injection barrier for their efficient transport to tackle the above unipolar issue. In addition, it is generally believed that the ambient stability of organic materials can be significantly improved via decreasing the LUMO level appropriately owing to declined trapped charge carries by water or oxygen in air atmosphere. ${ }^{[31]}$

Apart from energy level structures, morphology which refers to the microstructures of organic semiconducting layer is also of fundamental importance in the ambipolar performance and reliability of transistors. ${ }^{[32]}$ Various manufacture approaches and conditions, annealing temperatures, etc., could give rise to diverse molecular packing patterns and degrees of crystallinity and finally impact the device performance such as hole and electron mobilities. ${ }^{[3]}$ In order to form better morphology of organic film, several influence factors should be well modulated. First, organic semiconductors with various functional groups, alkyl side chains as well as attached positions can cause diverse molecular packing and thereby distinct morphology. ${ }^{[34]}$ Thus, suitable molecular engineering and hence well-designed molecular structure will play key roles in regulating the morphology. Second, the morphology also depends on various fabrication methods in which spin coating and vacuum deposition are the most commonly utilized approach. ${ }^{[35,36]}$ Therefore, by tuning the fabrication conditions such as heating temperature of evaporation as well as rotational speed and time, the morphology could be well controlled. Moreover, novel manufacture strategies for instance solution shearing are already exploited for changing the microstructures and the micropermutation of organic materials. Third, substrate modification is another way to adjust the morphology of organic materials fabricated via a solution-processing method and different sorts of SAMs such as octadecyl-trichlorosilane (OTS-18) have been utilized for modulating the surface energy of blocking dielectric and thereby the crystallinity of thin film. ${ }^{[37]}$ Favorable morphology not only can result in close molecular orbital overlap and intermolecular $\pi$-stacking and thereby better charge transport for high-performance ambipolar transistors but also is beneficial for the air stability and operational reliability of fabricated devices.

\subsection{Organic Small Molecules}

\subsubsection{Single Layer and Single Crystals}

One approach to fabricate ambipolar organic transistor is to investigate and synthesize single-component electrondonor and acceptor conjugated molecules (D-A-conjugated molecules) with tunable energy band alignments, enhanced $\pi$-conjugation and reduced inter-ring torsions by using appropriate donor (determine HOMO level) and acceptor (dominate LUMO energy) units. ${ }^{[38-43]}$ The electron withdrawing property resulted from introduced radicals (cyano group and fluorine) and extended conjugation length and augmented intermolecular $\pi-\pi$ stacking caused by some inserted organic functional groups in D-A molecules both can contribute to the ambipolar charge injection. ${ }^{[44,45]}$

In the past couple of years, various electron acceptors such as diketopyrrolopyrrole (DPP), ${ }^{[46]}$ naphthalene diimide (NDI) derivatives, ${ }^{[47]}$ isoindigos ${ }^{[48]}$ and $(E)$-[3,3'-bipyrrolylidene]$2,2^{\prime}\left(1 H, 1^{\prime} H\right)$-dione (BPD) ${ }^{[49]}$ have been mostly utilized for realizing high-performance ambipolar behavior. Among them, electron deficient DPP units form intense planar $\pi-\pi$ conjugated interactions and display large charge mobility (exceed $1 \mathrm{~cm}^{2} \mathrm{~V}^{-1} \mathrm{~s}^{-1}$ ). Besides, N-position of DPP units linked with two alkyl chains increases the solubility which is advantageous to fabricate devices. Cai et al. reported bidirectional $\pi$-extended DPP-based molecule (DPP-2F) with lower LUMO energy and high electron and hole mobilities up to 0.8 and $0.42 \mathrm{~cm}^{2} \mathrm{~V}^{-1} \mathrm{~s}^{-1}$ by reason of the introduction of 
electron withdrawing E-(1,2-difluorovinyl) moieties. ${ }^{[50]}$ However, the superior mobilities were tested under inert atmosphere and hence the practical application of this ambipolar transistor was limited. Later, a new air-stable molecule (DPP2T2P-2DCV) combining 2,5-dihydro-1,4-dioxo-3,6-di-2-thienyl1,4-diketopyrrolo[3,4-c]pyrrole derivative (DPP-2T) and the 4-(2,2-dicyanovinyl)phenyl radical was proposed by Bai et al. ${ }^{[51]}$ In their molecular design, DPP-2T functioned as an ambipolar semiconductor while cyano group (electron-withdrawing characteristic ) of dicyanovinyl in 4-(2,2-dicyanovinyl)phenyl radical was introduced to effectively lower the LUMO level and thereby augment electron injection from the contact electrodes, which was beneficial for both ambipolar charge transport and ambient stability. Besides, the introduced phenylene unit between DPP-2T and dicyanovinyl moieties could extend the conjugation length, which reduce the energy gap and facilitate intermolecular $\pi-\pi$ stacking. These improvements finally gave rise to excellent charge carrier mobilities of 0.015 (holes) and $0.168 \mathrm{~cm}^{2} \mathrm{~V}^{-1} \mathrm{~s}^{-1}$ (electrons) under ambient conditions. Recently, researchers also explored the novel ambipolar D-A small molecules by attaching different $\pi$-conjugating spacers including thiophene, bithiophene, benzothiophene, benzothiadiazole and thieno[3,2-b]thiophene (TT) to various common groups for instance DPP, NDI derivatives and isoindigo to regulate the intermolecular interactions and energy band structures. ${ }^{[52-59]}$ Lin et al. presented DPPderivatives with an A-D-A-D-A construction incorporating DPP core unit and planar bithiophene or TT electron donors. ${ }^{[60]}$ It was found that the implanted bithiophene or TT spacers with analogous material properties could lead to different energy levels and film morphologies and hence different ambipolar behaviors with high carrier mobilities can be achieved. In addition to the synthesis of novel D-A molecules, the device architectures (such as the position of source/drain contact electrodes) and the fabrication parameters (for instance thermal or solvent annealing temperatures) can improve the ambipolar charge transport. The transistor based on solution-processable D-A molecule composed of thiophene-dithienosilole-thiophene (Si1T) as donor units and DPP and carbonyl group as acceptors was demonstrated to exhibit larger charge carrier mobilities under higher thermal annealing temperatures and the usage of CVD-grown single-layer graphene (SLG) electrodes (Figure 3a,b), which could be explained from the smooth interfacial contact of the SLG source/drain electrodes. ${ }^{[61]}$

The electron-deficient NDI with large electron affinity and high thermal stability possesses intense $\pi$-stacking behavior and $\pi-\pi$ interactions between molecules (better charge transport characteristics) and has been utilized as chromophores, supramolecular structures and ambipolar organic semiconductors. Besides, the lower LUMO energy level caused by the diimide radical reduces the electron transport barrier and results in easy and air-stable electron injection. Wang et al. reported five conjugated molecules with the structure of acceptor-donor-acceptor (A-D-A) consisting of NDI as acceptors and various $\pi$-conjugated heterocyclic groups as donors (Figure 3c). ${ }^{[62]}$ The ultraviolet photoelectron spectroscopy (UPS) and cyclic voltammetry $(\mathrm{CV})$ characterizations revealed that the five compounds had approximately same LUMO levels (depend on the identical NDI moiety) and systematically augmenting HOMO levels (lie with differences of the donating strengths of donors and molecular conformation). The tunable bandgaps gave rise to the transition from unipolar (n-type) to ambipolar behavior with high electron and hole mobility values as large as 1.23 and $0.0074 \mathrm{~cm}^{2} \mathrm{~V}^{-1} \mathrm{~s}^{-1}$, respectively (Figure $3 \mathrm{~d}$ ). A similar donor-acceptor-donor (D-A-D) architecture where indeno[1,2b]fluorene-6,12-dione (IFDK) and indeno[1,2-b]fluorene-6,12-diylidene dimalononitrile (IFDM) acted as electron acceptors and $\alpha$-substituted bithiophene functioned as electron donors was designed by Ozdemir et al. (Figure 3e). ${ }^{[63]}$ Charge carrier mobilities of 0.01 and $0.13 \mathrm{~cm}^{2} \mathrm{~V}^{-1} \mathrm{~s}^{-1}$ for holes and electrons were observed in IFDM-based transistors at ambient condition due to stabilized electrons and holes in molecular backbones and good coplanarity and intense electron-accepting capability of IFDM cores with suitable size (Figure 3f). By comparing the air stability of charge transport in two organic semiconductors, it was found that the modification of the functional groups and hence the tuning of LUMO level dominated stable ambipolar performance under air atmosphere (Figure $3 \mathrm{~g}$ ). Inspired by the prominent charge transport performance of NDI, a novel electron-deficient thiophene-fused naphtho[2,3-b:6,7- $\left.b^{\prime}\right]$ dithiophene-4,5,9,10-diimide (NDTI) core structure was proposed and investigated in the applications of unipolar and ambipolar transistors. ${ }^{[53,64]}$ By connecting benzo[b]thiophene (BT) or naphtho[2,3-b]thiophene (NT) groups through $\alpha$-positions of the thiophene to the NDTI units demonstrated increasing balanced carrier mobilities $\left(0.16\right.$ and $0.25 \mathrm{~cm}^{2} \mathrm{~V}^{-1} \mathrm{~s}^{-1}$ for electrons and holes) (Figure $3 \mathrm{~h}-\mathrm{j})$. D-A molecules with favorable ambient-stability, simplified solution-processed method and reasonably balanced ambipolar mobilities are expected more with their developments.

In the last few years, ambipolar behavior has been achieved in indigo and isoindigo derivatives. Biological material indigo is a plant-derived dye and mainly used for coloring textiles. Indigo exhibits a remarkably large fusing point $\left(390-392{ }^{\circ} \mathrm{C}\right.$ ) and barely dissolves in conventional organic solvents owing to intense molecular interaction (hydrogen bonds). The intermolecular interactions of the hydrogen bonds $(-\mathrm{NH} . . . \mathrm{O}=\mathrm{C}-$ ) and $\pi$-skeletons dominate molecular packing structures as well as carrier transport characteristics. Indigo composed of amine radicals (donors) and carbonyl groups (acceptors) with longwavelength absorption (small bandgap) is favorable to realize ambipolar operation and presents air-stable well-balanced hole and electron mobilities of $0.01 \mathrm{~cm}^{2} \mathrm{~V}^{-1} \mathrm{~s}^{-1}{ }^{[65]}$ Recently, Pitayatanakul et al. put forward a sequence of bioinspired indigo derivatives replaced at the 5-position of Tyrian purple with halogen atoms ( $\mathrm{Br}$ and I) and phenyl groups (Figure 4a). ${ }^{[66,67]}$ The introduction of phenyl groups could enlarge the $\pi$-skeletons to realize parallel molecular packing while the adoption of $\mathrm{Br}$ and I atoms at the 5-position results in bathochromic shifts and thereby tunable bandgaps compared to substitutions at the 6-position. On the basis of structural analysis of the crystal by X-ray diffraction (XRD), atomic force microscopy (AFM), and single-crystal X-ray structure analysis, the authors found that 5-bromo substitution displayed roughly identical 1D molecular stacking in contrast to original indigo. 5-Iodine indigo exhibited supramolecular engineering and extremely large interchain interaction due to strong iodine-iodine interaction, and 5-phenyl substitution presented novel 2D brickwork and herringbone hybrid molecular packings. It was also discovered that 
(a)
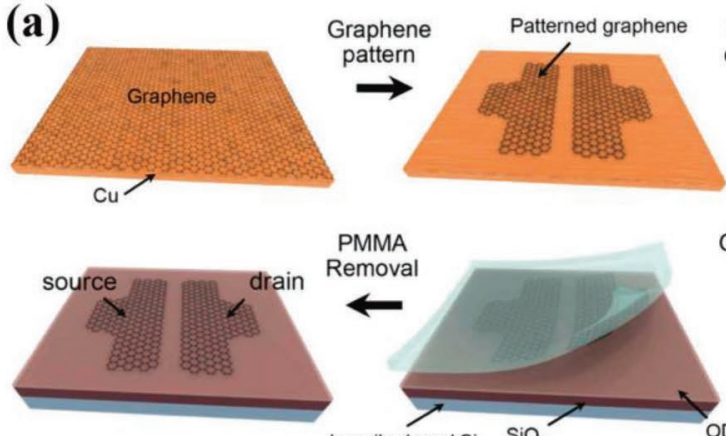

MMA

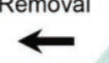

(c)

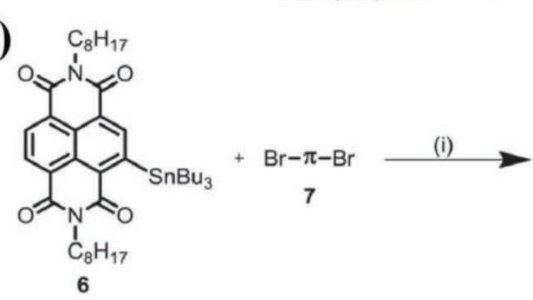<smiles>CCN1C(=O)c2ccc3c4c(ccc(c24)C1=O)C(=O)N(CC)C3=O</smiles>

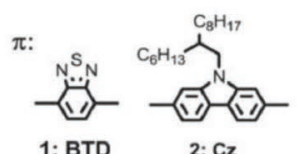
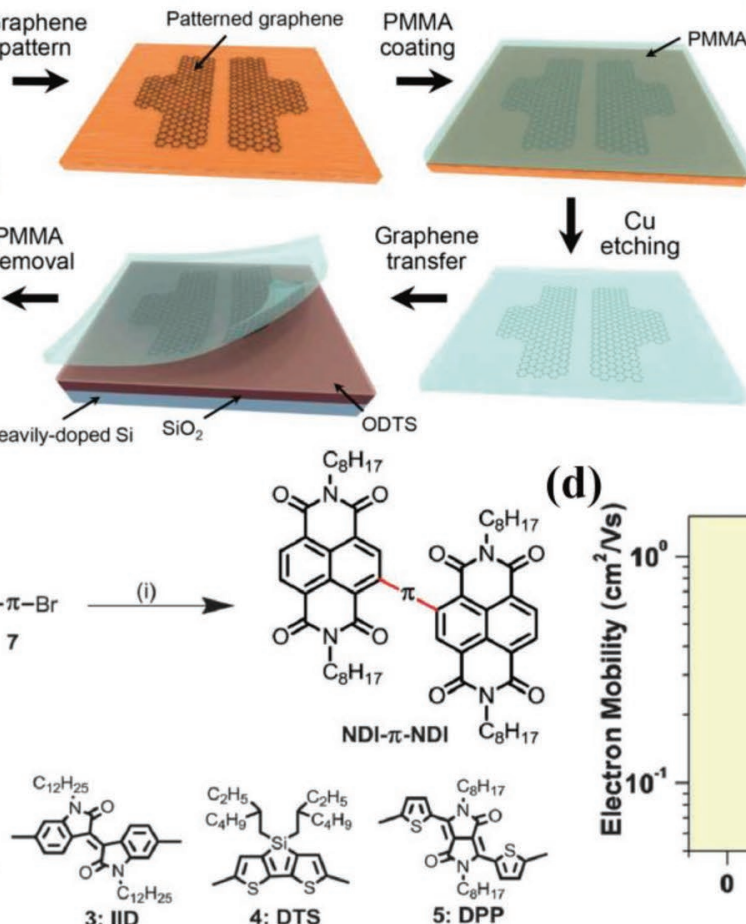

Graphene

(e)
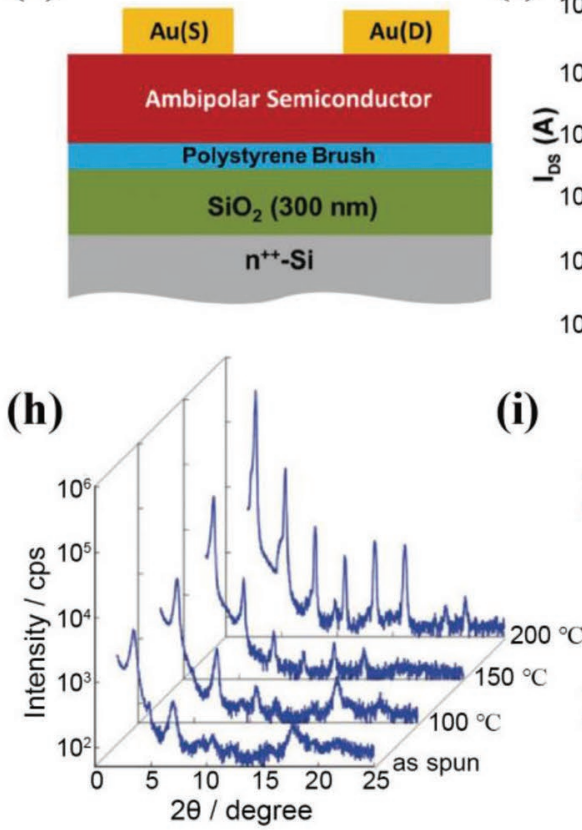

(f)
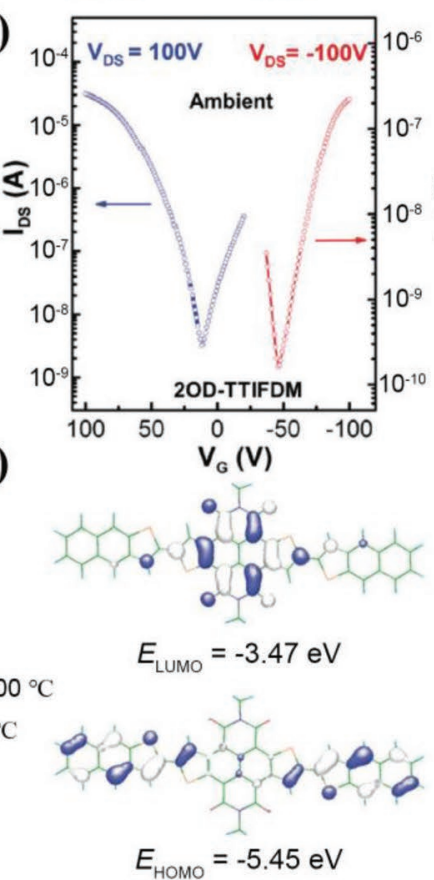
$\downarrow \underset{\text { etching }}{\mathrm{Cu}}$ etching

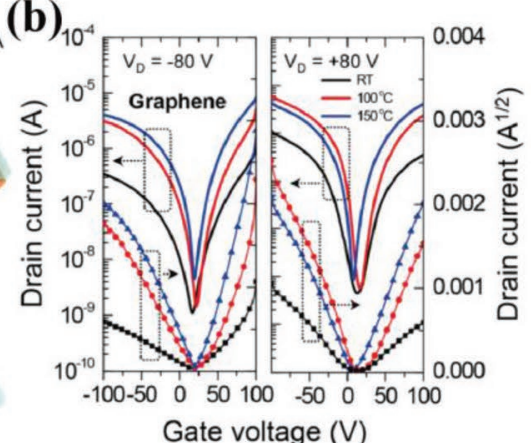

(d)

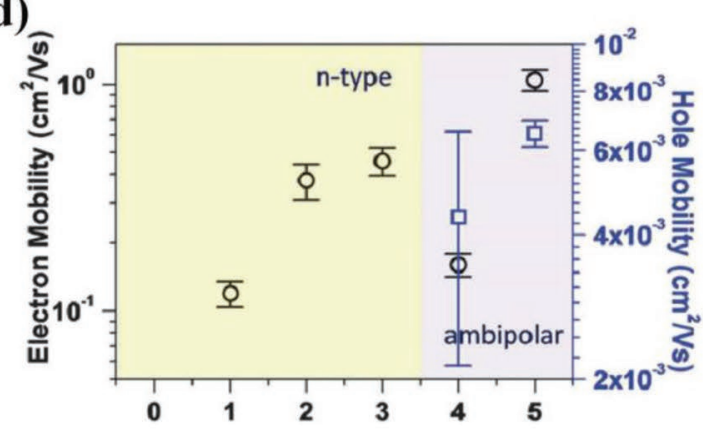

Figure 3. a) Schematic image of the fabrication processes of the SLG source-drain electrodes. b) Transfer curves (measured at a constant $V_{D}$ $(80$ or $-80 \mathrm{~V}))$ of the OTFTs on the basis of SilTDPP-EE-COC6 thin films which were annealed under three various temperatures $\left(25,100\right.$, or $\left.150{ }^{\circ} \mathrm{C}\right)$. Reproduced with permission. ${ }^{[61]}$ Copyright 2015, American Chemical Society. c) Synthesis routes of five organic NDI- $\pi$-NDI small molecules ((i) represents reaction conditions: $\mathrm{Pd}\left(\mathrm{PPh}_{3}\right)_{4}$, Cul, toluene, $110^{\circ} \mathrm{C}$ and final yield of $30-65 \%$ ). d) Derived mean hole (blue boxplots) and electron (black boxplots) mobilities of different NDI- $\pi$-NDI molecules. Reproduced with permission. ${ }^{[62]}$ Copyright 2016 , the Royal Society of Chemistry. e) Schematic diagram of top-contact bottom-gate ambipolar OFET architecture. f) Transfer characteristics of OFETs on the basis of 2OD-TTIFDM semiconductor (the red and blue curves represent $\mathrm{p}$ - and n-channels, respectively). g) Energy band alignments between HOMO/LUMO levels of two organic molecules and oxygen/water electrochemical redox couple (by comparing the air stability of charge transport in two organic semiconductors, it was found that the modification of the functional groups and hence the tuning of LUMO level dominated stable ambipolar performance under air atmosphere). Reproduced with permission. ${ }^{[63]}$ Copyright 2017, the Royal Society of Chemistry. h) XRD patterns of NDTI-BNT active layers. i) Calculated HOMO and LUMO of organic NDTI-BNT molecule. j) Transfer curve of transistors based on NDTI-BNT at air atmosphere (annealing temperature: $150^{\circ} \mathrm{C}$ ). Reproduced with permission. ${ }^{[64]}$ Copyright 2015, the Royal Society of Chemistry. 
(a)

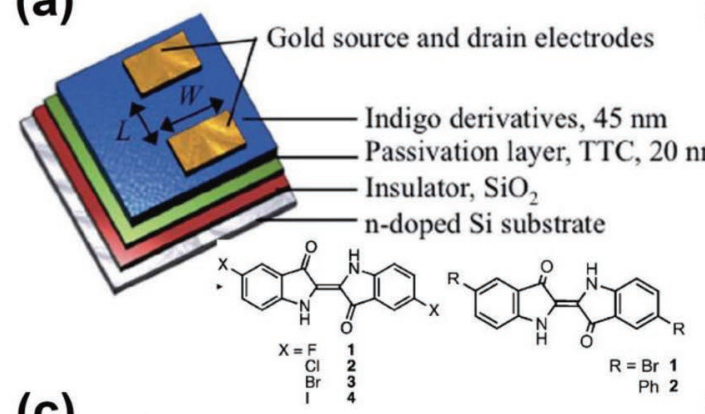

(c)

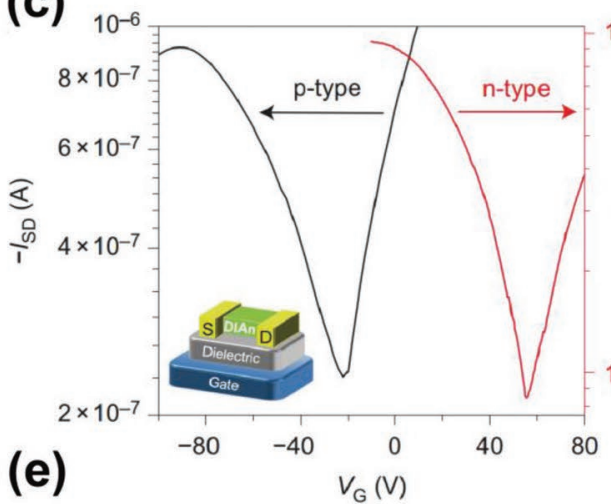

(d)
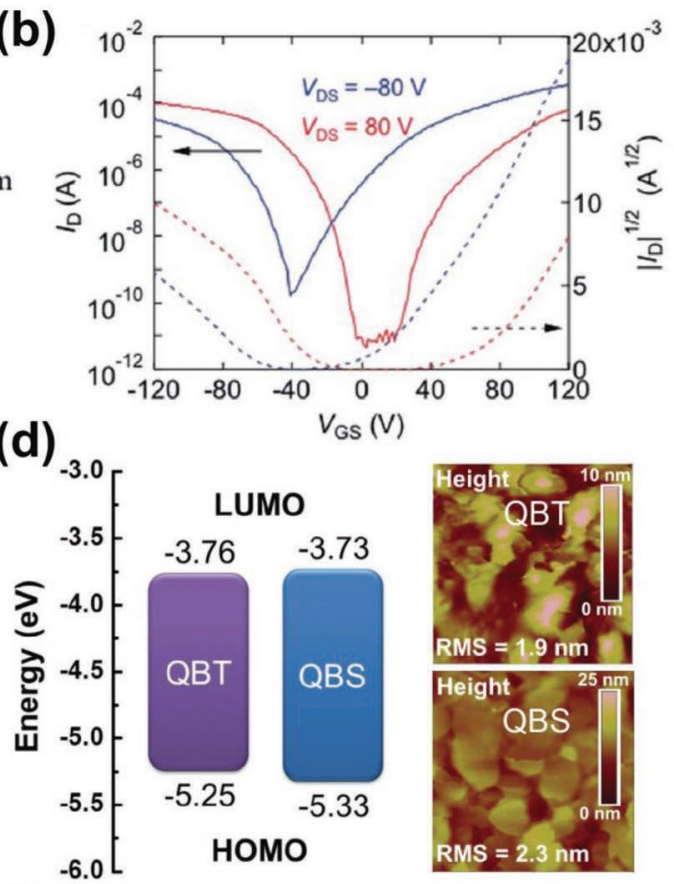

(f)

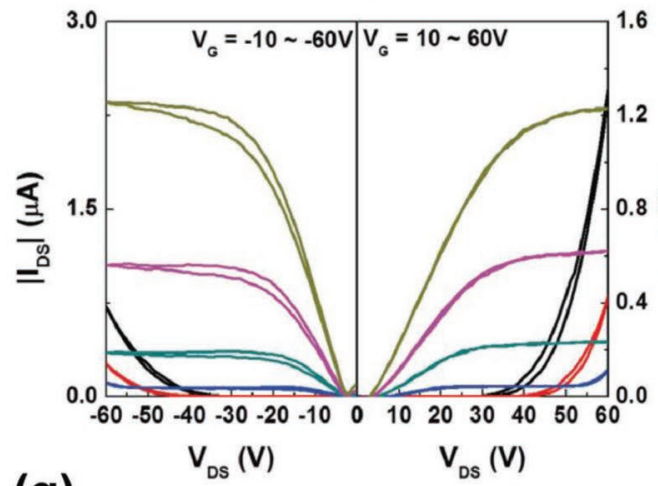

(g)

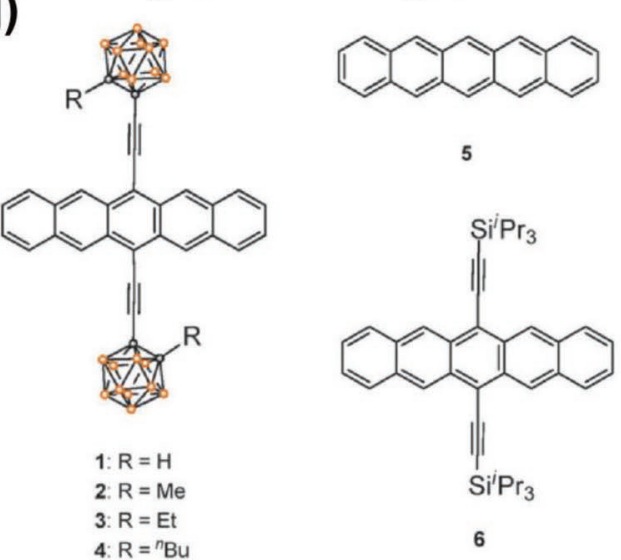

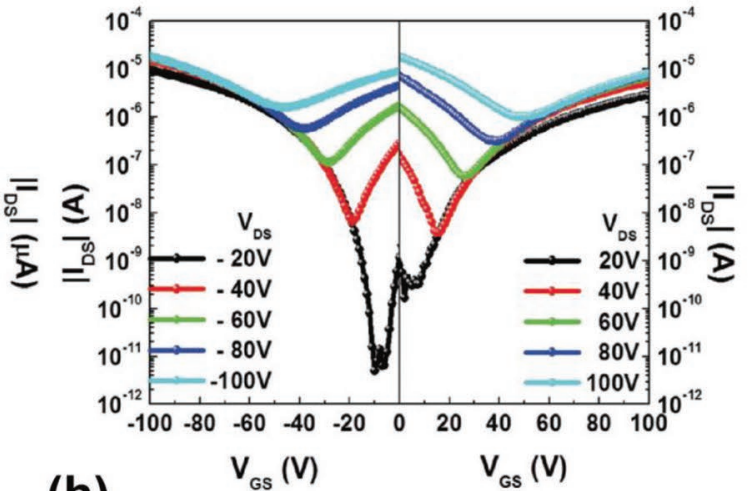

(h)

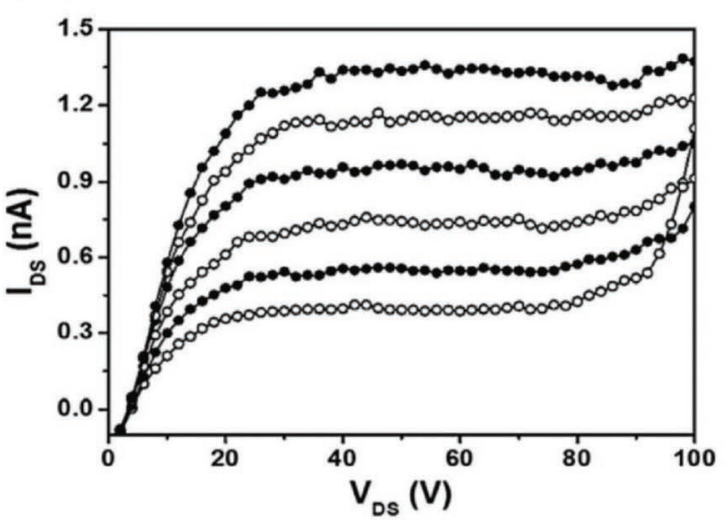

Figure 4. a) Structures of bottom-gate top-contact indigo-based OFETs (up) and various indigo derivatives (bottom) (W and $L$ in the figure refer to 1000 and $100 \mu \mathrm{m}$, respectively). b) Transfer curves of OTFTs based on 5,5'-diphenylindigo. Reproduced with permission. ${ }^{[66]}$ Copyright 2014 , the Royal Society of Chemistry. c) Transfer characteristics of DIAn-based OFETs operated under $\mathrm{p}$ - and $\mathrm{n}$-channel mode. For positive gate bias, $V_{S D}$ is $80 \mathrm{~V}$ while for negative gate bias, $V_{S D}$ is $-100 \mathrm{~V}$. Reproduced with permission. ${ }^{[71]}$ Copyright 2016, Nature Publishing Group. d) HOMO and LUMO levels as well as AFM height images (annealing temperature: $170^{\circ} \mathrm{C}$ ) of QBT and QBS. Output e) and transfer f) characteristic curves of OFETs on the basis of QBS molecules. Reproduced with permission. ${ }^{[1]}$ Copyright 2015, Wiley-VCH. g) Structures of different pentacene derivatives. h) N-type output characteristics of transistors based on 4 . Reproduced with permission. ${ }^{[72]}$ Copyright 2015, the Royal Society of Chemistry. 
the arrangement of the indigo cores of 5-iodine and 5-phenyl substitutions was basically perpendicular to the substrate for purpose of acquiring maximum mobilities. Among them, 5,5'-diphenylindigo showed highest well-balanced ambipolar charge mobilities of 0.56 and $0.95 \mathrm{~cm}^{2} \mathrm{~V}^{-1} \mathrm{~s}^{-1}$ for holes and electrons, respectively (Figure 4b).

Electron-deficient isoindigo with symmetrical lactam architecture is an isomer of indigo and also observed to show ambipolar charge transport phenomena. Nonplanar isoindigo sometimes presents brickwork structure on account of the intramolecular steric repulsion. Other than the reported brickwork architecture, Ashizawa et al. investigated the pristine unsubstituted isoindigo with ambipolar charge mobilities exceeding $0.01 \mathrm{~cm}^{2} \mathrm{~V}^{-1} \mathrm{~s}^{-1}$ and found another two polymorphs including stacking and nonplanar molecular structures. ${ }^{[68]}$ According to the exhibited charge transport polarities of a sequence of isoindigo derivatives with electron-withdrawing radicals, the authors concluded that the minimal effective HOMO level for hole transport was $-5.7 \mathrm{eV}$. Besides, it was found that 6,6'-diphenylisoindigo presented hole mobilities of $0.037 \mathrm{~cm}^{2} \mathrm{~V}^{-1} \mathrm{~s}^{-1}$ and electron mobilities of $0.027 \mathrm{~cm}^{2} \mathrm{~V}^{-1} \mathrm{~s}^{-1}$ because of perpendicular molecular arrangements onto the substrate. In addition to the halogen atoms and phenyl radicals, cyano groups with intense electron-withdrawing characteristics were also introduced to isoindigo cores to reduce the LUMO level of the molecules. The transistors based on the dicyanated isoindigo displayed unipolar charge transport by modifying the dielectric with self-assembled monolayers (SAMs) of $n$-tetradecylphosphonic acid (TPA) whereas it was interestingly noted that the device showed good ambipolar performance under the adoption of $12,12,13,13,14,14,15,15,16,16,17,17,18,18,18$-pentadecylfluorooctadecylphosphonic acid (FOPA) SAMs onto the insulating layer probably due to polarization effect of the electronegative fluorine atoms. ${ }^{[69]}$ Different substituents and substitutional positions together with modified insulating layers via various SAMs provide more possibilities to realize better performance ambipolar transistors on the basis of isoindigo derivatives. ${ }^{[70]}$

Some new classes of ambipolar semiconducting materials exhibiting amphoteric redox process have been proposed by researchers lately. The amphoteric redox behavior signifies concurrent achieved oxidation and reduction phenomena in single organic molecules and dominates transport processes of electrons (holes) into LUMO (HOMO) level. Rudebusch et al. designed and characterized a novel chemical robust biradical molecule, diindeno[ $b, i]$ anthracene (DIAn) with open-shell character and high stability under air atmosphere and elevated temperatures. ${ }^{[71]}$ The Raman spectroscopy, CV, and magnetic susceptibility characterization confirmed amphoteric redox characteristic and thermally accostable triplet excited state. The fabricated transistors based on vapor deposited DIAn semiconducting layer and octyltrichlorosilane-treated dielectrics displayed thermal-stable balanced ambipolar charge mobilities of about $10^{-3}$ to $10^{-2} \mathrm{~cm}^{2} \mathrm{~V}^{-1} \mathrm{~s}^{-1}$ (Figure $4 \mathrm{c}$ ) originated from simultaneous cooperative dual pseudoquinoidal (for electron transport) and pseudoaromatic (for hole transport) architectures. Hwang et al. also reported two new quinoidal molecules, quinoidal bithiophene (QBT) and quinoidal biselenophene (QBS) with planar structures beneficial for $\pi-\pi$ stacking and carrier transport, extended $\pi$-electron delocalization favorable for lower energy gap and amphoteric redox behavior (Figure 4d). ${ }^{[11]}$ The authors revealed that although their electrochemical characteristics and HOMO/LUMO levels were basically the same (depended on quinoidal architectures), QBS comprising selenophene presented much larger carrier mobilities $(0.055$ and $0.021 \mathrm{~cm}^{2} \mathrm{~V}^{-1} \mathrm{~s}^{-1}$ for holes and electrons) in contrast to QBT on account of intense intermolecular interaction induced by polarized Se atoms and hence highly augmented crystallinity after thermal annealing (Figure 4e,f). In addition, recent studies found that modifying inserted substituents especially alkyl side chains could generate various molecular packings and thereby may convert unipolar charge injection to ambipolar behavior. Guo et al. demonstrated the successful transition from p-type semiconducting characteristics to ambipolar charge transport by incorporating $o$-carborane group $1-(\mathrm{HC} \equiv \mathrm{C})-2-\mathrm{R}-1,2-\mathrm{C}_{2} \mathrm{~B}_{10} \mathrm{H}_{10}$ ( $\mathrm{R}$ is alkyl chain with different lengths) into $\pi$-conjugated pentacene to reduce HOMO/LUMO levels (Figure 4g,h). ${ }^{[2]} \mathrm{Xu}$ et al. also discovered that both unipolar and ambipolar operations could be observed among three dithiophene-4,9-dione-containing semiconducting molecules with identical conjugated core and analogous energy levels but various $n$-alkyl chains, which was explained by diverse molecular packing and microstructures existed within the thin layers on the basis of XRD results. ${ }^{[73]}$ These strategies and findings bring new avenues to search for ambipolar semiconducting materials.

Apart from aforementioned single component-modified small molecular semiconductors with narrow energy gaps for ambipolar carrier transport, another method to achieve ambipolar operation is to blend two semiconducting materials (one is p-type and the other is n-type). ${ }^{[74]}$ This can be achieved by mixed solution processing approach or vacuum coevaporation of two semiconductors, resulting in various microstructures. Different combinations and ratios of the two components both have a great influence on the film morphology/microstructures and hence the mobility values, which remains a challenge in fine tuning the blends' morphology.

Most molecular blends form microphase-separated $\mathrm{p}-\mathrm{n}$ network, known as bulk heterojunction (BHJ). Cheng et al. demonstrated that via blending [2-phenylbenzo $\left[d, d^{\prime}\right]$ thieno[3,2- $b ; 4,5$ $b^{\prime}$ ]dithiophene (P-BTDT) : 2-(4-n-octyl- phenyl)benzo[d, $\left.d^{\prime}\right]$ thieno[3,2-b;4,5- $b^{\prime}$ ]dithiophene (OP-BTDT)] (p-channel; named M-BTDT) and $\mathrm{C}_{60}$ (n-channel) with optimized fractions gave rise to a $\mathrm{BHJ}$ ambipolar transistor with favorable structured network as well as well-balanced hole $\left(0.03 \mathrm{~cm}^{2} \mathrm{~V}^{-1} \mathrm{~s}^{-1}\right)$ and electron mobilities $\left(0.02 \mathrm{~cm}^{2} \mathrm{~V}^{-1} \mathrm{~s}^{-1}\right)$ (Figure 5a,b). ${ }^{[26]}$ Very recently, the largest well-balanced charge mobilities $\left(0.83 \mathrm{~cm}^{2} \mathrm{~V}^{-1} \mathrm{~s}^{-1}\right.$ for holes and $0.37 \mathrm{~cm}^{2} \mathrm{~V}^{-1} \mathrm{~s}^{-1}$ for electrons) of ambipolar transistors based on solution-processable organic small molecules were attained by Vegiraju et al., with semiconductor mixtures of dicyanomethylene substituted dithienothiophene-quinoid (DTTQ-11; n-type) and p-type DbT-TTAR (composed of tetrathienothiophene core and two linked bithiophene groups) in identical blending ratios (Figure $5 \mathrm{~d}$ ). ${ }^{[75]}$ The authors also found that various mixing ratios could induce transition between unipolar and ambipolar transport, which presented a viable approach to modulate the molecular blends and further tune microstructures and carrier injection performance (Figure 5c).

Charge-transfer (CT) complexes can also be good candidates for ambipolar transport, where the mixing fractions of 
(a)

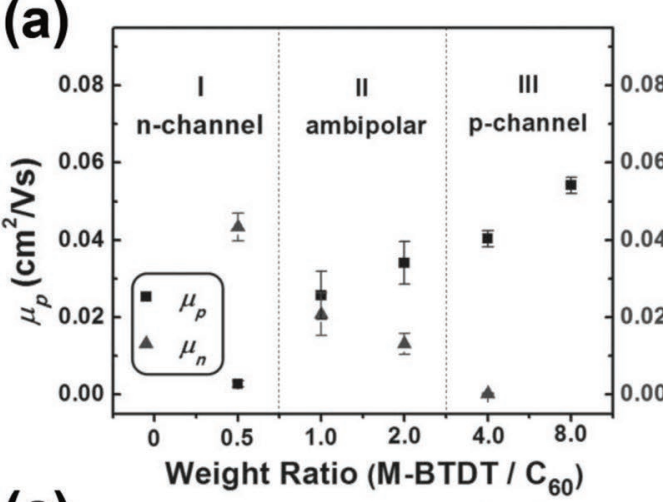

(c)

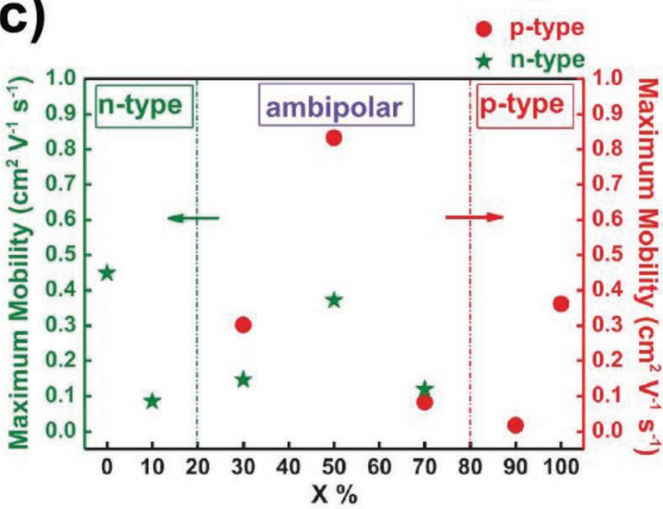

(e)

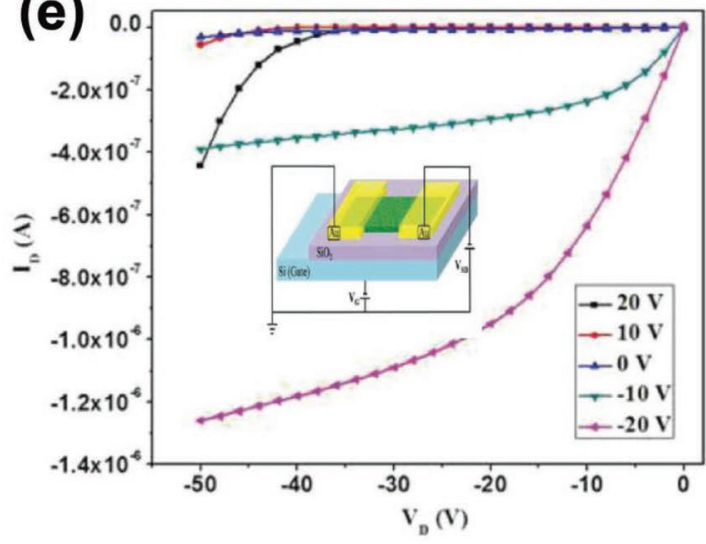

(b)

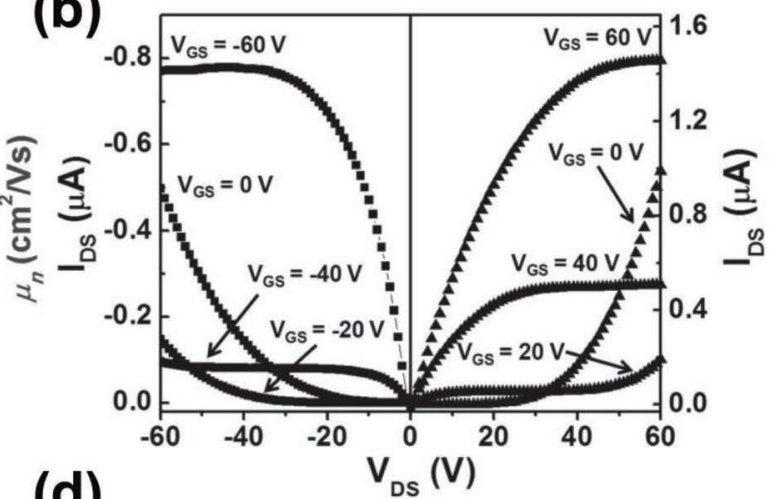

(d)

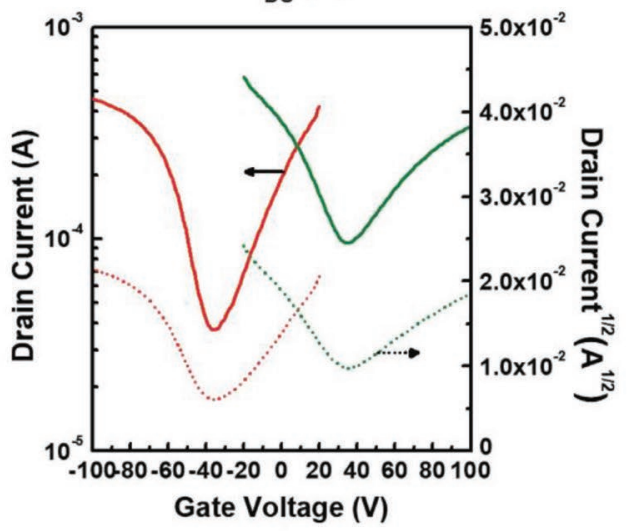

(f)

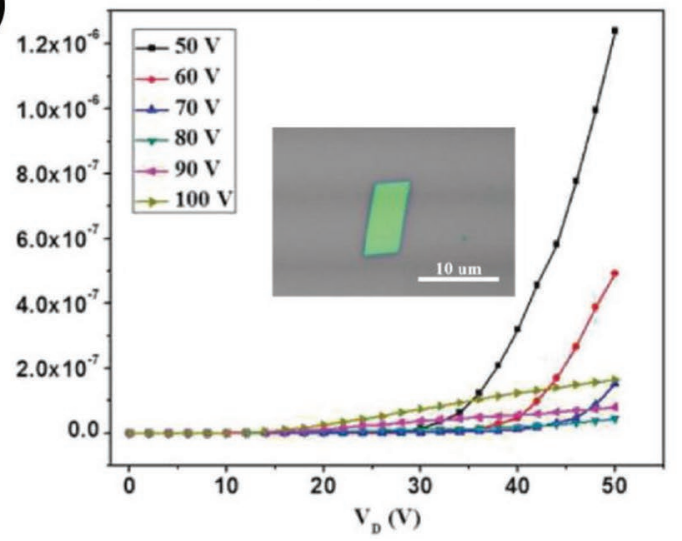

Figure 5. a) The dependence of hole and electron mobilities of transistors on various weight ratios between $M$-BTDT and $\mathrm{C}_{60}$ (Three operation models including p-channel, n-channel, and ambipolar processes can be observed in $\mathrm{BHJ}$ ambipolar OTFTs). b) Output curves based on $\mathrm{M}$ - $\mathrm{BTDT} / \mathrm{C}_{60} \mathrm{BHJ}_{\mathrm{J}}$ with blending ratio of 1:1. Reproduced with permission. ${ }^{26]}$ Copyright 2014, Wiley-VCH. c) Maximum charge carrier mobilities of OFETs measured under nitrogen atmosphere with respect to different ratios of DbT-TTAR. d) Transfer characteristics of OFETs on the basis of DbT-TTAR/DTTQ-11 mixed semiconductor with equal ratio. Reproduced with permission. [75] Copyright 2018, Wiley-VCH. Output curves of transistors based on DPTTA-TCNQ cocrystal nanosheets measured under positive f) and negative e) source-drain biases, respectively. The insets describe the transistor structure e) and optical image of single DPTTA-TCNQ cocrystal nanosheet f). Reproduced with permission. ${ }^{[79]}$ Copyright 2014, Wiley-VCH.

donor and acceptor molecules are usually the same and the $\pi$-stacks together with intermolecular interactions conduce to their different optoelectronic properties for instance ferroelectricity, electrical conductivity and photovoltaic effects. ${ }^{[76,77]}$ There are several reports about ambipolar transistors on CT complexes such as combinations of 2,5-dimethyl- $N, N^{\prime}$-dicyanop-quinonediimine (DMDCNQI; acceptor) and dibenzopyrrolo$[3,2-b]$ pyrrole (DBPP; donor ${ }^{[78]}$. The best ambipolar D-A complexes incorporating equal-ratio donor molecules meso-diphenyl tetrathia[22]annulene[2,1,2,1] (DPTTA) and acceptor semiconductors 4,8-bis(dicyanomethylene)-4,8-dihydrobenzo[1,2- $\quad b: 4,5$ $b^{\prime}$ ]-dithiophene (DTTCNQ) were demonstrated by Qin et al.[79] On the grounds of crystallographic analysis and quantum calculations, the authors concluded that extended $\pi$-conjugated D-A molecules with enhanced intermolecular interactions led to existence of super exchange effect and formation of quasi-2D transport network, which contributed to remarkably ambipolar performance with large mobilities of 0.77 and $0.24 \mathrm{~cm}^{2} \mathrm{~V}^{-1} \mathrm{~s}^{-1}$ 
for holes and electrons, respectively (Figure 5e,f). Recently, donor (silylethynylated pentacene)-acceptor (silylethynylated tetraazapentacene) organic blending semiconductors with a novel form of solid solution were proposed. ${ }^{[0]}$ The transistors based on this mixture with mixing fraction of 1:1 exhibited ambipolar balanced charge mobilities comparable to previously mentioned ambipolar transistors based on BHJ and CT complexes.

Single crystal transistors have been extensively explored to achieve ambipolar charge transport phenomena owing to their superior characteristics such as free of grain boundaries and defects and molecular ordering within extensive region. ${ }^{[81-85]}$ Crystallization of oligomers is usually utilized to prepare organic single crystals in which the main interaction force is van der Waals interaction. ${ }^{[86]}$ These interactions are derived from electronic movements inside the organic molecules and thereby generated fluctuations of dipole moments. The fabricated organic single crystals maintain the electrical characteristics of original component organic molecules due to inherent weak van der Waals force. ${ }^{87,88]}$ Micro- and nanostructured single crystals are mainly fabricated using physical vapor deposition (PVD) or solution-based methods. Nonetheless, PVD approach is mainly applied to the highly thermal-stable conjugated small molecules and has its own limitations (large energy consumption). ${ }^{[89]}$ Thus, simple solution-processed self-assembly accompanied with intense molecular $\pi-\pi$ stacking is expected to be a favorable method for constructing the organic single crystal of various materials. ${ }^{[0]}$ For the solution-based approach, lots of techniques such as thermal annealing, ${ }^{[91]}$ inkjet printing (antisolvent crystallization) ${ }^{[2]}$ and dip coating ${ }^{[93]}$ have been utilized to enhance the crystallinity and pattern the micro- and nanostructures of organic single crystal. However, the consumed time and power of the device fabrication are still large and remain a big challenge. Recently, Watanabe et al. reported a novel method based on microcrystallization in capillaries (one of soft lithography techniques) to pattern and crystallize needle-shaped QQT(CN)4 crystal utilizing PDMS molds in one step. ${ }^{[94]}$ The concentration of QQT $(\mathrm{CN}) 4$, the shape of PDMS molds together with the depth and width of capillaries could be modulated to tune the morphologies of the QQT $(\mathrm{CN}) 4$ microline arrays. The fabricated bottom-gate top-contact transistor demonstrated representative ambipolar charge transport but relatively small hole and electron mobilities compared to previous reported QQT(CN)4 devices. However, from the fabrication technique viewpoints, this new method with the merits of decreased energy consumption, fabrication time and equipment cost can accelerate the development of organic ambipolar transistors.

Plenty of organic single crystal transistors exhibit unipolar performance (mostly p-type), which is in direct contravention of the measurement results (both two types of charge carriers can move in organic single crystals) based on time of flight (ToF) and time-resolved microwave conductivity (TRMC). ${ }^{[95]}$ The aforementioned evident discrepancy is caused by the reality that the charge transport properties of single crystal transistors not only depend on the intrinsic properties of the organic semiconductors but also rely on other important factors, i.e., source and drain metal electrodes and interface characteristics between dielectric layer and semiconducting film. Particularly, owing to the Fermi-level pinning of semiconducting material on source and drain electrodes, the energy band structures between the work functions of source and drain metal electrodes and the HOMO and LUMO levels of the organic crystals are extremely significant in deciding which type of charge is dominant. As the metal work function is nearer to the HOMO level of semiconductor, the energy barrier of holes is smaller and hence the transistors display hole-dominated performance (p-type, i.e., pentacene and rubrene). ${ }^{[96,97]}$ Some reported organic single crystals such as 2,7-dioctyl[1]benzothieno[3,2-b][1]benzothiophene (C8-BTBT, $\left.31.3 \mathrm{~cm}^{2} \mathrm{~V}^{-1} \mathrm{~s}^{-1}\right)^{[92]}$ and 2,9-didecyldinaphtho[2,3-b:2,3f]thieno[3,2-b]thiophene $\left(\mathrm{C}_{10} \text {-DNTT, } 12.5 \mathrm{~cm}^{2} \mathrm{~V}^{-1} \mathrm{~s}^{-1}\right)^{[98]}$ show large hole mobilities of exceeding $10 \mathrm{~cm}^{2} \mathrm{~V}^{-1} \mathrm{~s}^{-1}$. Up to now, p-type single crystal transistors based on poly(10,12-pentacosadiynoic acid) (poly-PCDA) present largest hole mobility of $\approx 42.7 \mathrm{~cm}^{2} \mathrm{~V}^{-1} \mathrm{~s}^{-1} \cdot{ }^{[99]}$ Nevertheless, as the metal work function is nearer to the LUMO level of semiconductor, the injection barrier of electrons is much lower and thereby the transistors function as n-type devices (i.e., organic single crystal transistors on the basis of 7,7,8,8-tetracyanoquinodimethane (TCNQ) or perylene derivatives). ${ }^{[100,101]}$ By far, two representative n-type organic single crystals are 2,5-difluoro-7,7,8,8-tetracyanoquinodimethane (F2TCNQ $)^{[102]}$ and 4Cl-TAP ${ }^{[103]}$ which exhibit highest electron mobilities of 25 (temperature: $150 \mathrm{~K}$ ) and $27.8 \mathrm{~cm}^{2} \mathrm{~V}^{-1} \mathrm{~s}^{-1}$, respectively.

Ambipolar charge transport property was first realized in organic single crystal transistors on the basis of $\mathrm{Cu}$ - and Fe-phthalocyanine ${ }^{[104]}$ and rubrene was also demonstrated to display ambipolar behavior later (Figure 6a-c). ${ }^{[05]}$ From then on, more and more organic single crystals with large or small energy gaps have been found to show ambipolar electrical performance. ${ }^{[106-109]} \mathrm{Up}$ to now, with regard to device configuration, only top-contact architecture has been exploited for efficient electron injection, which might be explained that the defects generated during thermal evaporation process may help electrons inject into organic single crystals. With respect to metal electrode, $\mathrm{Au}, \mathrm{Ag}, \mathrm{Al}$, and $\mathrm{Ca}$ are more commonly utilized and preparation of dense Ca electrodes can give rise to excellent electron injection behavior. ${ }^{[110,111]}$ Besides, asymmetric source and drain electrodes (i.e., $\mathrm{Au}$ and $\mathrm{Ca}$ ) are also used to achieve ambipolar charge transport (Figure 6d). ${ }^{[107,112,113]}$ In addition to the aforementioned several factors, another crucial point to realize ambipolar transport is that the capture sites of electrons at the interface of blocking layer and semiconducting layer should be minimized. Researchers usually employ thin nonhydroxyl polymeric film (i.e., poly-methyl methacrylate (PMMA) and crosslinked polyvinyl pyrrolidone (PVP)) to passivate the interface. ${ }^{[114]}$ It was also found that both the morphology and the remanent impurity within passivation film significantly affected the amount of the residual trap sites at the interface. ${ }^{[115]}$

Due to the challenges in achieving ambipolar behavior in transistors based on organic single crystals, scientists have proposed several other strategies to realize ambipolar charge transport. One of them is utilizing organic single crystals with deeper LUMO and HOMO to enhance the ability of electron injection. ${ }^{[116]}$ In addition to this method, one can also insert a thin layer (electron injection layer) between electrodes and active layer to improve the capability of electron injection (Figure 6e). ${ }^{[117]}$ Furthermore, ambipolar charge transport can also be obtained by exploiting cocrystals on the basis of donor and acceptor organic molecules instead of single 
(a)

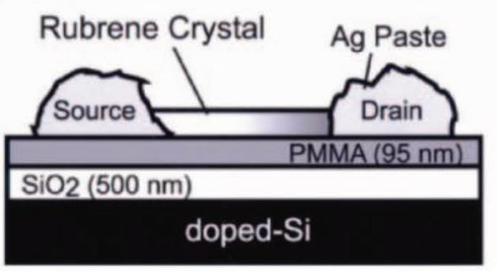

(b)

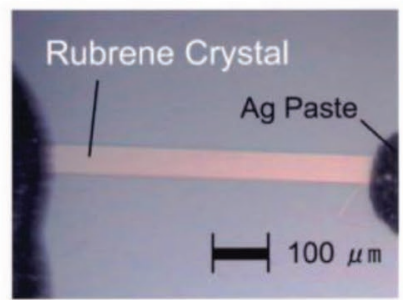

(c)

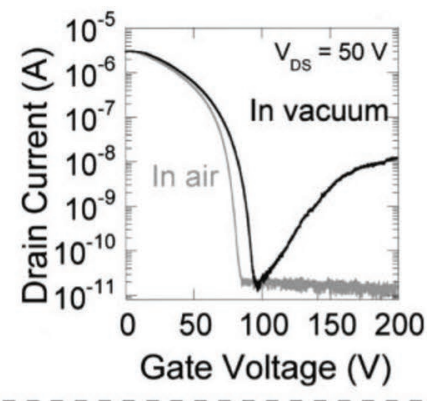

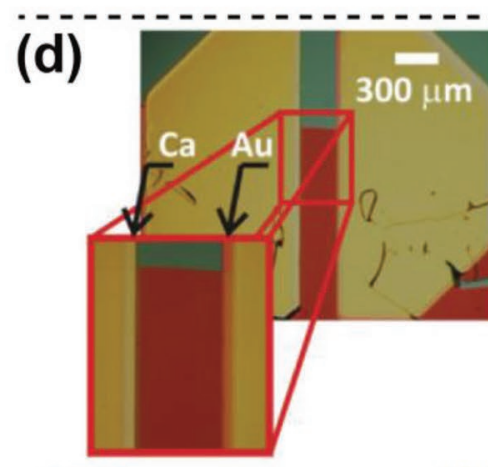
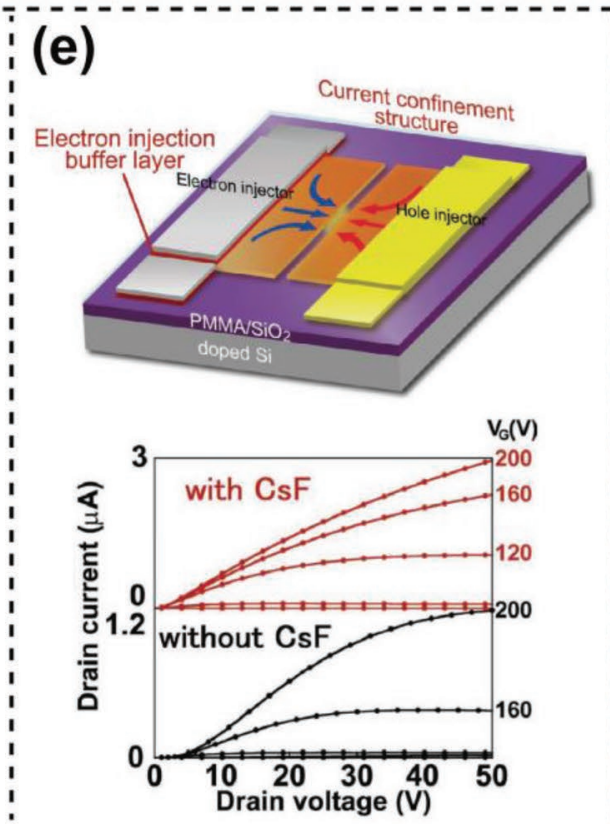
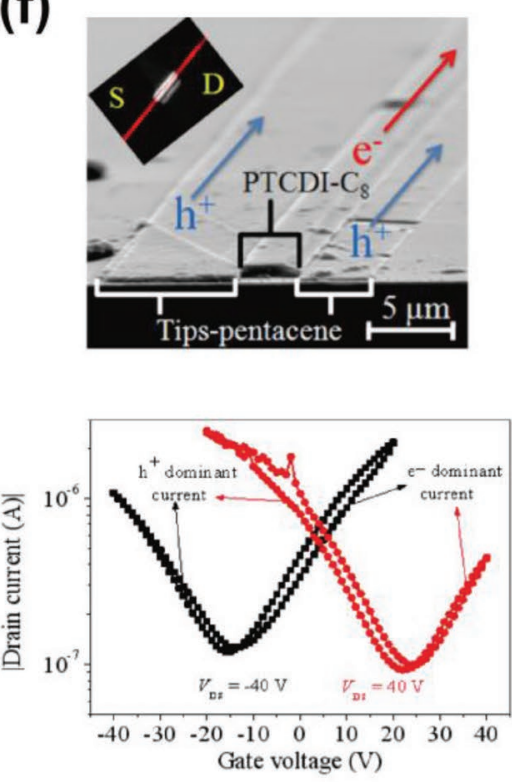

Figure 6. Schematic illustration a), optical image b), and typical transfer curves c) of organic single crystal transistor based on rubrene. The black and gray lines in (c) represent vacuum condition and air atmosphere, respectively. Reproduced with permission. ${ }^{[105]}$ Copyright 2006, American Institute of Physics. d) Optical image of organic single crystal transistor with $\mathrm{Ca}$ and Au asymmetric source-drain electrodes; representative output curves of ambipolar transistor on the basis of $\alpha, \omega$-bis (biphenylyl)terthiophene single crystals. The red and blue lines represent $\mathrm{p}$ - and $\mathrm{n}$-channels, respectively. Reproduced with permission. ${ }^{[107]}$ Copyright 2009, Wiley-VCH. e) Schematic diagram of the design mechanism of enhanced electron injection by inserting an additional electron injection layer between electrodes and active layer; typical output curves of ambipolar organic single crystal transistor based on BP3T. The red and black lines represent transistor with cesium fluoride (CsF) and transistor without CsF, respectively. Reproduced with permission. ${ }^{[17]}$ Copyright 2012, Wiley-VCH. f) SEM top-viewed image of vertically cut OFETs based on TPT architecture; transfer characteristics of ambipolar OFETs based on TPT crystals. Reproduced with permission. ${ }^{120]}$ Copyright 2014, American Chemical Society.

crystals based on a type of molecule. ${ }^{[118,119]}$ On account of the fact that the electronic characteristics of cocrystals lie with the energy band alignment of their HOMO and LUMO levels as well as their particular molecular arrangement, thus the constituent donor and acceptor organic molecules need to be chosen leerily to realize effective ambipolar transport. Recently, Shim et al. demonstrated air-stable high-performance transistors based on laterally stacked organic microcrystal wires with the structure of 6,13-bis(triisopropylsilylethynyl)pentacene (Tips-pentacene/p-type semiconductor)/ $N, N^{\prime}$-dioctyl-3,4,9,10perylenedicarboximide (PTCDI-C $8 / n$-type semiconductor)/ Tips-pentacene (TPT architecture) through solution-processing approach ("capillary tube method") (Figure 6f). ${ }^{[120]}$ By repeated optimization of solution concentrations of the semiconductors, the direct contact between ambipolar crystals and the metal electrodes was expected to induce direct injection of holes and electrons for well-balanced charge carrier mobilities.
Up to now, the largest hole $\left(22 \mathrm{~cm}^{2} \mathrm{~V}^{-1} \mathrm{~s}^{-1}\right)$ and electron $\left(5.0 \mathrm{~cm}^{2} \mathrm{~V}^{-1} \mathrm{~s}^{-1}\right)$ mobilities were reported in single-crystal organic rubrene semiconductors by Kanagasekaran et al. (Figure $7 \mathrm{a}-\mathrm{c}) .^{[121]}$ The authors proposed a novel concept of source-drain injection electrodes on the basis of structure of metal layer ( $\mathrm{Ca}$ or $\mathrm{Au}$ )/polycrystalline organic semiconductors/ tetratetracontane, which displayed high injection efficiencies of both two types of charge carriers (Figure 7d,e). This new electrode design could be implemented onto other different semiconducting materials and is attractive for the achievement of ambipolar transistors with better electrical performance.

In summary, in order to achieve effective ambipolar charge transport in single crystal transistors, careful design of organic single crystals, source-drain metal electrodes, modification layer (hole/electron injection layer) between electrodes and active layer as well as polymeric buffer layer between blocking dielectric and single crystal is crucial. Compared to unipolar single crystal 
(a)

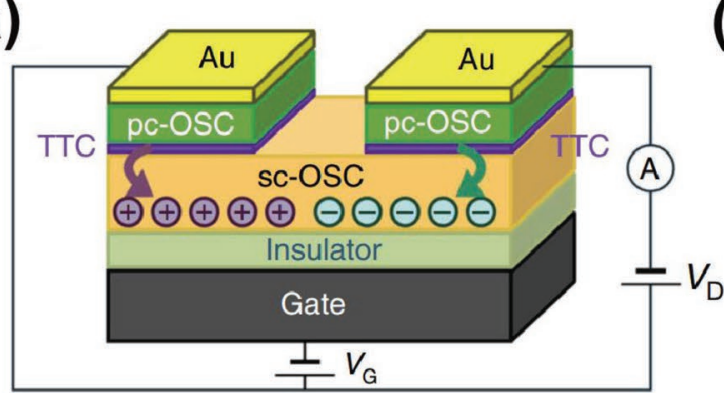

(b)

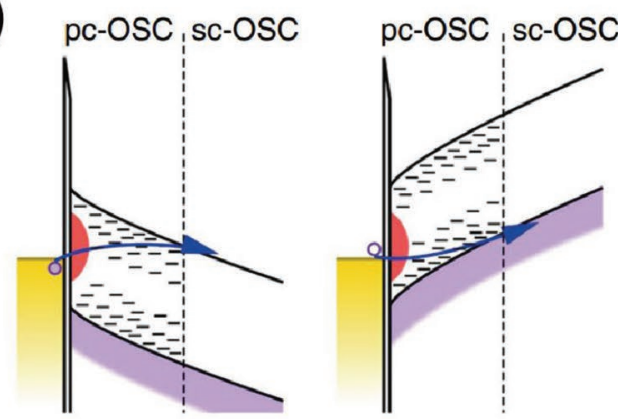

(d)

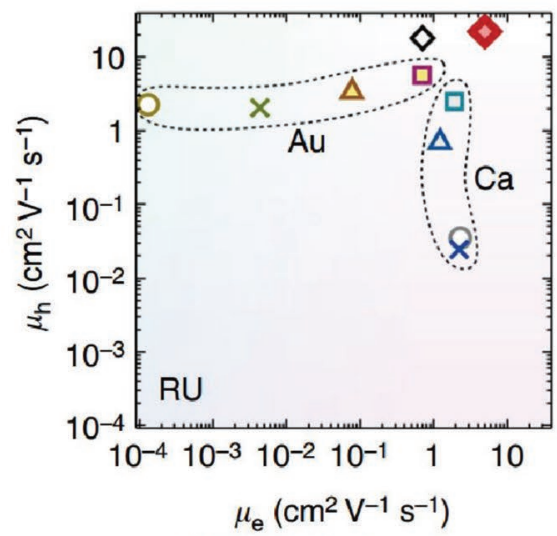

(f)

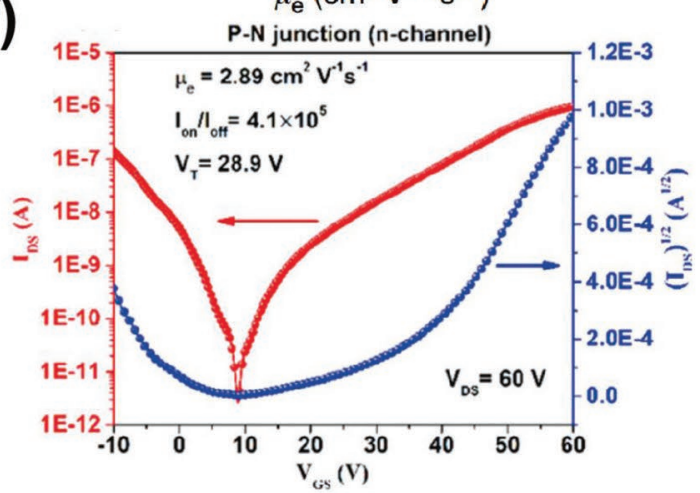

(c)
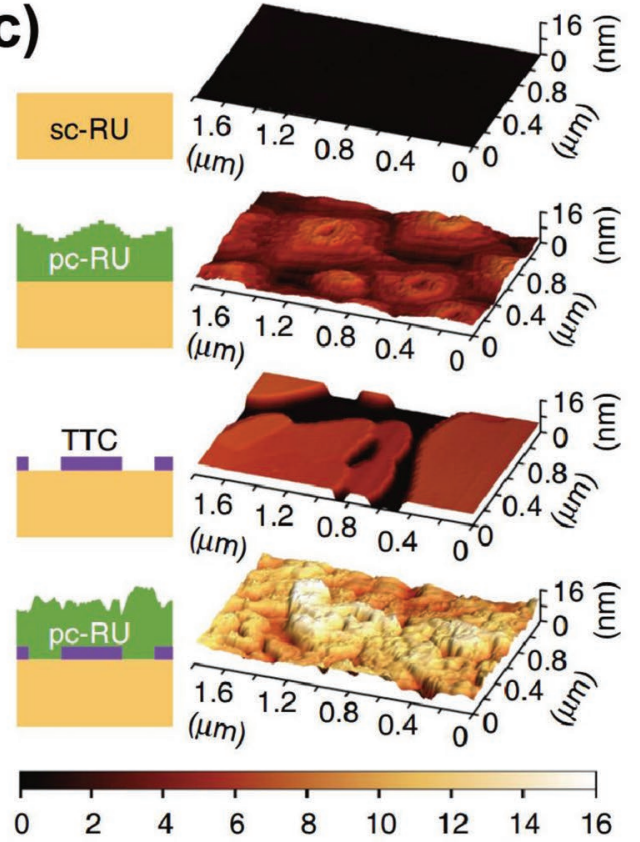

Height $(\mathrm{nm})$

(e)
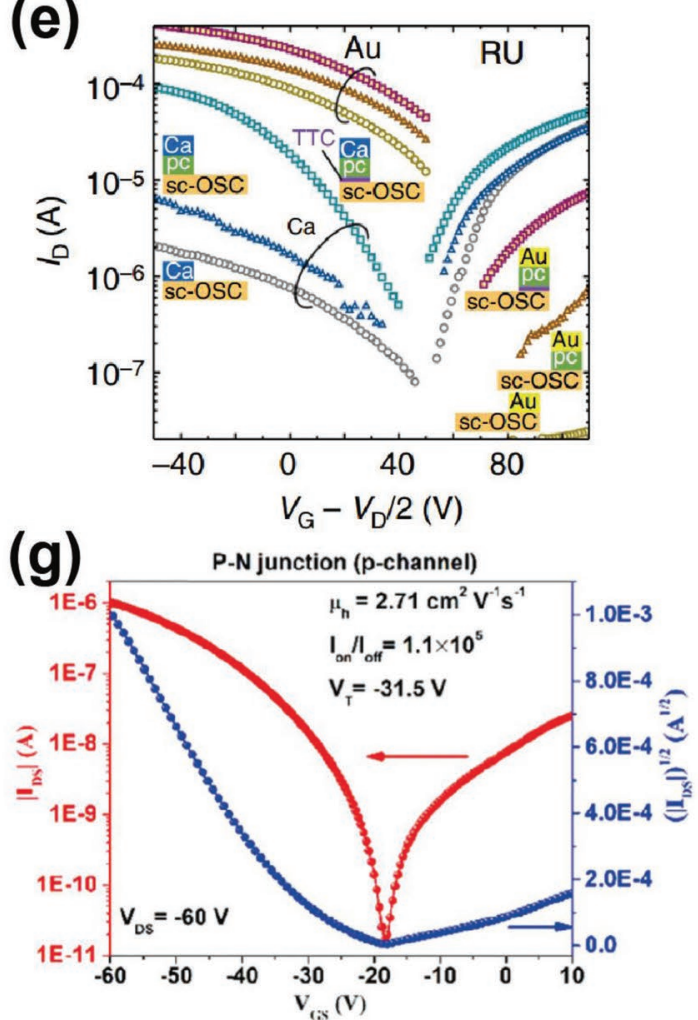

Figure 7. a) Schematic 3D FET structure using novel source-drain electrodes metal (Au)/ polycrystalline organic semiconductor (pc-OSC)/tetratetracontane (TTC) to inject both two types of charge carriers. b) Electron (left) and hole (right) injection mechanism of aforementioned novel electrodes revealed by their energy band structures. c) AFM morphologies of single crystal rubrene (sc-RU), pc-RU/sc-RU, TTC/sc-RU as well as pc-RU/TTC/sc-RU, respectively (from top to bottom). Electron and hole mobilities d) as well as transfer characteristics e) of single-crystal rubrene-based transistors with different electrodes. Yellow-to-magenta and blue-to cyan symbols represent $\mathrm{Au}$ and $\mathrm{Ca}$ electrodes, respectively. Circles, crosses, triangles and squares represent no interlayer, CsF interlayer, pc-OSC interlayer as well as pc-OSC/TTC interlayer, respectively. Red and black diamonds indicate the largest mobilities of single-crystal rubrene-based transistors reported in this article and other literatures. Reproduced with permission. ${ }^{[121]}$ Copyright 2017, Nature Publishing Group. Transfer characteristics of OFETs on the basis of TIPS-PEN/ $\mathrm{C}_{60} \mathrm{SCH}$ ) operated under n-channel f) and p-channel g) regimes, respectively. Reproduced with permission. ${ }^{[135]}$ Copyright 2018, American Chemical Society. 
transistors, charge carrier mobilities of ambipolar transistors are relatively small, which remains a big challenge and might be solved by exploiting thinner or monolayer organic single crystals. ${ }^{[122]}$ In addition, contrast between top-contact and bottomcontact configurations and how to realize ambipolar transport in bottom-contact single crystal transistors require further exploring. Furthermore, challenges still exist in how to grow and pattern organic single crystals extensively. ${ }^{[123]}$ The growth direction, homogeneity as well as size of organic single crystals need to be deliberated. Moreover, the operational stability and retention capability of ambipolar organic single crystal transistors need further improving. Further development of single crystal transistors can be achieved by tackling aforementioned scientific issues.

\subsubsection{Bilayer Structure}

In order to obtain ambipolar performance, transistors with two sequentially deposited $\mathrm{p}$ - and $\mathrm{n}$-type semiconducting active materials with bilayer structures were first reported by Dodabalapur and co-workers in 1995. ${ }^{[124]}$ They put forward the combinations of hole-transporting $\alpha$-hexathienylene and electron-conducting $\mathrm{C}_{60}$ bilayer semiconductors via vacuum evaporation and observed relatively balanced hole and electron mobilities. Both the energy band alignments of the two small molecules and the deposition sequences were found to be crucial for bipolar conduction. This work has arisen extensive investigations of bilayer ambipolar transistors with diverse combinations of small molecules ${ }^{[125]}$ such as pentacene $/ \mathrm{C}_{60},{ }^{[126]} \mathrm{CuPc} / \mathrm{F}_{16} \mathrm{CuPc}^{[127]}$ $\mathrm{SnCl}_{2} \mathrm{Pc} / \mathrm{CuPc},{ }^{[128]}$ pentacene/PTCDI-C $8,{ }^{[129]}$ etc.

Vasimalla et al. fabricated flexible air-stable ambipolar transistors in which plastic overhead projector (OHP) sheets, laminated Al foil, C-PVA (crosslinked PVA) and combinations of p-type $\mathrm{CuPc}$ film and n-type $\mathrm{Br}_{2} \mathrm{PTCDI}-\mathrm{C} 18$ layer were used as bendable substrates, gate electrode, insulating and semiconducting materials, respectively. ${ }^{[130]}$ By exerting low operating electrical bias of $\pm 3 \mathrm{~V}$, matched charge mobilities $\left(1.2 \times 10^{-2} \mathrm{~cm}^{2} \mathrm{~V}^{-1} \mathrm{~s}^{-1}\right.$ for electrons and $5.5 \times 10^{-3} \mathrm{~cm}^{2} \mathrm{~V}^{-1} \mathrm{~s}^{-1}$ for holes) were obtained. Gao et al. combined hole-conducting vacuum deposited CuPc layer (bottom layer) and electron-conducting complex (TFPP) Eu[Pc(OPh) 8 $\mathrm{Eu}\left[\mathrm{Pc}(\mathrm{OPh})_{8}\right][\mathrm{TFPP}=$ dianion of 5,10,15,20-tetrakis ( $p$-fluorophenyl)porphyrin; $\mathrm{Pc}(\mathrm{OPh})_{8}=$ dianion of 2,3,9,10,16,17,23,24-oct a(phenoxy)phthalocyanine] (top layer) by means of bilayer semiconductors and found that the hole and electron mobilities were significantly increased up to 0.16 and $0.3 \mathrm{~cm}^{2} \mathrm{~V}^{-1} \mathrm{~s}^{-1}$ in contrast to single triple-deck complex semiconducting layer due to prominent elevation of the molecular ordering of the complex in bilayer architecture. ${ }^{27]}$ Chang et al. demonstrated well-balanced carrier mobility values of $1-1.1 \mathrm{~cm}^{2} \mathrm{~V}^{-1} \mathrm{~s}^{-1}$ by utilizing $\alpha, \omega$-diperfluorohexylquaterthiophene (DFH-4T) and dinaphtho[2,3-b:2', $\left.3^{\prime}-f\right]$ thieno[3,2-b]thiophene (DNTT) as n- and p-type components, respectively. ${ }^{[131]}$ The device presented a coarse and nonplanar heterojunction. Based on the electrical and structural analysis, the authors explained that microstructural compatibility between these two components was beneficial for the formation of interconnected continuous network, large contact areas and efficient electron injection, which led to superior ambipolar behavior.
In the bilayer structure, various design factors such as the selection of $\mathrm{p}$ - and n-type materials, deposition order, relative film thickness, source/drain contacts and in particular $\mathrm{p} / \mathrm{n}$ interface have influences on the ambipolar performance. Among them, the regulation of the morphology of the active layers and hence the contact interface between them is more important for ambipolar charge transport on account of the appearance of carrier accumulation regions at interface. Several solution-based strategies have been proposed to achieve effective active layers and interface. One way is to insert an intermediate layer between the $\mathrm{p}$ - and $\mathrm{n}$ - active layers. ${ }^{[132]}$ Another approach is to deposit two films separately onto two different substrates and then move arbitrary one layer onto the other layer. ${ }^{[133]}$ The orthogonal solvents based spin-coating method is widely adopted to reduce the damage to the bottom layer after the sequential deposition. ${ }^{[134]}$ Up to now, among various bilayer ambipolar transistors based on small molecules, Zhao et al. manufactured singlecrystal transistors with highest well-balanced carrier mobilities of more than $2.5 \mathrm{~cm}^{2} \mathrm{~V}^{-1} \mathrm{~s}^{-1}\left(2.9 \mathrm{~cm}^{2} \mathrm{~V}^{-1} \mathrm{~s}^{-1}\right.$ for electrons and $2.7 \mathrm{~cm}^{2} \mathrm{~V}^{-1} \mathrm{~s}^{-1}$ for holes) by utilizing single-crystalline $\mathrm{p}-\mathrm{n}$ heterojunctions (SCHJs) comprising p-type 6,13-bis(triisopropyls ilylethynyl)pentacene (TIPS-PEN) and n-type $\mathrm{C}_{60}$ (Figure $7 f, g$ ), which stimulates more exploration of new SCHJ systems for high-performance ambipolar transistors. ${ }^{[135]}$

The energy levels, electrical performance, processing methods as well as device structures of recent selected organic small molecules and single crystals are summarized in Table 1 . The chemical structures of them are listed in Figure 8. As we retrospect, diverse novel organic small molecules with various functional groups, alkyl side chains as well as different attached positions of them have been extensively synthesized and exhibit ambipolar behaviors. However, for single-component semiconducting molecules, the displayed electron and hole mobilities are still relatively low $\left(\leq 1 \mathrm{~cm}^{2} \mathrm{~V}^{-1} \mathrm{~s}^{-1}\right)$ meanwhile their ambient stability is poor. Therefore, the relation between chemical structure and ambipolar performance should be more investigated and deeply understood and well-designed small molecules with better ambipolar charge transport are required. For molecular blends, how to control the morphology of them remains challenging. The fabrication parameters of experimental process such as heating temperature, concentration, evaporation speed, etc., both should be adjusted, controlled and optimized. Novel manufacture strategies except for conventional solution-processing method or vacuum coevaporation need further exploration. As to bilayer architecture, as above, deposition order, relative film thickness, source/drain contacts and in particular $\mathrm{p} / \mathrm{n}$ interface both could impact their electrical properties and hence measures should be taken to modulate and optimize these conditions. In particular, more approaches should be researched to form better $\mathrm{p}-\mathrm{n}$ interface and hence effective charge accumulation region.

\subsection{Polymers}

\subsection{Single-Component Polymers}

Polymer materials with advantages of superior viscosity, thermal, mechanical and simple processing characteristics have been proverbially employed in photovoltaics (PV), transistors, 
Table 1. Summary of electrical properties, performance, processing methods and device architectures of ambipolar transistors one the basis of semiconducting organic small molecules and single crystals.

\begin{tabular}{|c|c|c|c|c|c|c|c|}
\hline \multirow[t]{2}{*}{ Classification } & \multirow[t]{2}{*}{$\begin{array}{c}\text { Organic } \\
\text { semiconductors }\end{array}$} & \multirow[t]{2}{*}{ HOMO $[\mathrm{eV}]$} & \multirow[t]{2}{*}{$\begin{array}{l}\text { LUMO } \\
{[\mathrm{eV}]}\end{array}$} & \multicolumn{2}{|c|}{$\begin{array}{l}\text { Mobilities } \\
{\left[\mathrm{cm}^{2} \mathrm{~V}^{-1} \mathrm{~s}^{-1}\right]}\end{array}$} & \multirow[t]{2}{*}{$\begin{array}{l}\text { Processing methods and } \\
\text { semiconductor configurations }\end{array}$} & \multirow[t]{2}{*}{ Ref. } \\
\hline & & & & $\mu_{\mathrm{e}}$ & $\mu_{\mathrm{h}}$ & & \\
\hline \multirow{6}{*}{$\begin{array}{l}\text { Organic small molecules } \\
\text { DPP-based derivatives }\end{array}$} & S1 & -5.39 & -3.70 & 0.80 & 0.42 & Spin coating & [50] \\
\hline & & & & & & Single layer & \\
\hline & S2 & -5.36 & -3.81 & 0.168 & 0.015 & $\begin{array}{l}\text { Spin coating } \\
\text { Single layer }\end{array}$ & [51] \\
\hline & S3 & -5.27 & -3.68 & 0.02 & 0.16 & $\begin{array}{l}\text { Spin casting } \\
\text { Single layer }\end{array}$ & [46] \\
\hline & S4 & -5.15 & -3.52 & 0.015 & 0.011 (SLG electrodes) & $\begin{array}{l}\text { Spin coating } \\
\text { Single layer }\end{array}$ & [61] \\
\hline & S5 & -5.36 & -3.80 & 0.065 & 0.031 & $\begin{array}{l}\text { Spin coating } \\
\text { Single layer }\end{array}$ & [60] \\
\hline \multirow[t]{2}{*}{ NDI-based derivatives } & S6 & -5.47 & -3.94 & 1.23 & 0.0074 & $\begin{array}{l}\text { Spin coating } \\
\text { Single layer }\end{array}$ & [62] \\
\hline & S7 & -5.45 & -3.47 & 0.16 & 0.25 & $\begin{array}{l}\text { Spin coating } \\
\text { Single layer }\end{array}$ & [64] \\
\hline \multirow[t]{2}{*}{ Indigo derivatives } & s8 & -5.44 & -3.58 & 0.85 & 0.42 & $\begin{array}{l}\text { Vacuum evaporation } \\
\text { Single layer }\end{array}$ & [67] \\
\hline & s9 & -5.56 & -3.91 & 0.95 & 0.56 & $\begin{array}{l}\text { Vacuum evaporation } \\
\text { Single layer }\end{array}$ & [66] \\
\hline \multirow[t]{3}{*}{ Isoindigo derivatives } & $S 10$ & -5.44 & -3.42 & 0.027 & 0.037 & $\begin{array}{l}\text { Vacuum evaporation } \\
\text { Single layer }\end{array}$ & [68] \\
\hline & S11 & -5.85 & -3.88 & 0.11 & 0.045 & $\begin{array}{l}\text { Vacuum deposition } \\
\text { Single layer }\end{array}$ & [69] \\
\hline & S12 & -5.11 & -3.32 & 0.13 & 0.12 & $\begin{array}{c}\text { Thermal deposition } \\
\text { Single layer }\end{array}$ & [70] \\
\hline \multirow[t]{7}{*}{ Other small molecules } & $S 13$ & -5.27 & -3.82 & 0.004 & 0.002 & $\begin{array}{l}\text { Vapor deposition } \\
\text { Single layer }\end{array}$ & [71] \\
\hline & S14 & -5.33 & -3.73 & 0.021 & 0.055 & $\begin{array}{l}\text { Spin coating } \\
\text { Single layer }\end{array}$ & [11] \\
\hline & S15 & -5.09 & -3.86 & 0.09 & 0.08 & $\begin{array}{l}\text { Vacuum deposition } \\
\text { Single layer }\end{array}$ & [39] \\
\hline & S16 & -5.12 & -3.41 & 0.22 & 0.03 & $\begin{array}{l}\text { Vacuum deposition } \\
\text { Single layer }\end{array}$ & [73] \\
\hline & S17 & -5.60 & -3.65 & 0.12 & 0.02 & $\begin{array}{l}\text { Solution shearing } \\
\text { Single layer }\end{array}$ & [40] \\
\hline & S18 & -5.17 & -3.71 & 0.58 & 0.054 & $\begin{array}{l}\text { Vacuum deposition } \\
\text { Single layer }\end{array}$ & [49] \\
\hline & S19 & -5.49 & -4.23 & 0.13 & 0.01 & $\begin{array}{l}\text { Solution shearing } \\
\text { Single layer }\end{array}$ & [63] \\
\hline \multirow[t]{3}{*}{ Single crystals } & S20-S21 (1:1) & - & - & 0.47 & 1.57 & $\begin{array}{l}\text { Drop casting } \\
\text { Single crystal }\end{array}$ & [42] \\
\hline & S22 & -5.62 & -3.11 & 0.019 & 0.023 & $\begin{array}{l}\text { Lamination } \\
\text { Singe crystal }\end{array}$ & [41] \\
\hline & S23 & -5.91 & -3.16 & 0.13 & 0.085 & $\begin{array}{l}\text { Lamination } \\
\text { Single crystal }\end{array}$ & [84] \\
\hline
\end{tabular}


Table 1. Continued.

\begin{tabular}{|c|c|c|c|c|c|c|c|}
\hline \multirow[t]{2}{*}{ Classification } & \multirow[t]{2}{*}{$\begin{array}{c}\text { Organic } \\
\text { semiconductors }\end{array}$} & \multirow[t]{2}{*}{$\mathrm{HOMO}[\mathrm{eV}]$} & \multirow[t]{2}{*}{$\begin{array}{l}\text { LUMO } \\
{[\mathrm{eV}]}\end{array}$} & \multicolumn{2}{|c|}{$\begin{array}{l}\text { Mobilities } \\
{\left[\mathrm{cm}^{2} \mathrm{~V}^{-1} \mathrm{~s}^{-1}\right]}\end{array}$} & \multirow[t]{2}{*}{$\begin{array}{l}\text { Processing methods and } \\
\text { semiconductor configurations }\end{array}$} & \multirow[t]{2}{*}{ Ref. } \\
\hline & & & & $\mu_{\mathrm{e}}$ & $\mu_{\mathrm{h}}$ & & \\
\hline & S24 & -6.01 & -3.26 & 2.50 & 2.10 & $\begin{array}{l}\text { Physical vapor transport } \\
\text { Single crystal }\end{array}$ & [81] \\
\hline & S25-S26 & - & - & 2.9 & 2.7 & $\begin{array}{l}\text { Drop casting } \\
\text { Single crystal }\end{array}$ & [135] \\
\hline & S27 & -6.69 & -2.28 & 0.5 & 0.4 & $\begin{array}{l}\text { Drop casting } \\
\text { Single crystal }\end{array}$ & [85] \\
\hline & S27 & - & - & 0.083 & 0.17 & $\begin{array}{l}\text { Microcrystallization in capillary } \\
\text { Single crystal }\end{array}$ & [94] \\
\hline & S28 & -4.85 & -2.58 & 5.0 & 22 & $\begin{array}{l}\text { Lamination } \\
\text { Single crystal }\end{array}$ & [121] \\
\hline
\end{tabular}

light-emitting diodes, logic circuits, sensors, etc. ${ }^{[136-143]}$ Particularly, D-A polymers that possess noncovalent intramolecular interactions for efficient $\pi$-conjugation and close stacking ensuring intermolecular charge hopping have been extensively investigated.[144-154] The conjugated polymers consisting of donors including selenophenes and thiophenes and acceptors comprising DPPs, ${ }^{[155,156]}$ NDIs, ${ }^{[157-164]}$ isoindigos, ${ }^{[48,165-167]}$ thiadiazolopyridine, naphthalenedicarboximide, and benzothiadiazole are mainly synthesized via Stille, Suzuki, and direct arylation polycondensation. Among diverse acceptor units, isoindigos and DPPs have been investigated a lot for ambipolar operation.

Isoindigos made up of the aromatic groups and ketopyrrole cores possess high electron-deficient nature and coplanar structure, which is advantageous to design semiconducting copolymers with superior charge transport performance. ${ }^{[168-170]}$ Isoindigo was first utilized for constructing donor materials by Reynolds and co-workers in 2010 ${ }^{[171]}$ and later the first isoindigo-based copolymers with large charge mobilities (more than $0.7 \mathrm{~cm}^{2} \mathrm{~V}^{-1} \mathrm{~s}^{-1}$ for holes) were put forward by Pei and co-workers. ${ }^{[172]}$ During the past few years, scientists have created several novel isoindigo-based acceptor units (namely, BDOPV, NBDOPV, INDF, and DIID) by inserting various functional groups inside isoindigos to extend their conjugation lengths and reduce LUMO levels and energy gaps. ${ }^{[153,173-177]}$ Zhou et al. reported solution-processable copolymers by employing BDOPV as strong acceptor units for lower LUMOs and thieno[3,2-b]thiophene as donor moieties for regulating HOMOs (Figure 9a). ${ }^{[178]}$ The fabricated transistors showed air-stable balanced hole and electron mobilities of 1.70 and $1.37 \mathrm{~cm}^{2} \mathrm{~V}^{-1} \mathrm{~s}^{-1}$, which was stemmed from high crystallinity and ordered packing of the semiconducting layer (Figure 9b). Jiang et al. proposed a new isoindigo-based electron-deficient derivative, isoindigo[7,6g]isoindigo (DIID) and copolymerized it with electron-donating (E)-1,2-bis(thiophen-2-yl)ethene moieties via Stille polycondensation. ${ }^{[179]}$ The obtained bottom-gate/top-contact transistors demonstrated ambipolar performance with high mobilities (1.79 and $0.087 \mathrm{~cm}^{2} \mathrm{~V}^{-1} \mathrm{~s}^{-1}$ for holes and electrons, respectively) (Figure 9c).

On the other hand, there is a torsion between benzene group and its neighboring unit on account of the $\mathrm{C}-\mathrm{H}$...H-C steric effects within isoindigo. Therefore, researchers also tried to modify the isoindigo core to tune their HOMOs/LUMOs, intensity of $\pi-\pi$ interaction and the steric hindrance by substituting thiophene, thienothiophene, benzothiophene or thieno[3,2-b] [1]benzothiophene for the benzene ring of isoindigos and synthesizing fused isoindigos. ${ }^{[180]}$ In 2012, Ashraf and co-workers first constructed novel thienoisoindigo by replacing the benzene group with thiophene ring and obtained balanced charge mobilities. ${ }^{[181]}$ Later, Meager et al. proposed a new thieno[3,2-b] thiophene isoindigo acceptor moiety combined with benzothiadiazole donor unit and demonstrated ambipolar characteristics with hole mobility of $0.4 \mathrm{~cm}^{2} \mathrm{~V}^{-1} \mathrm{~s}^{-1}$ and electron mobility of $0.7 \mathrm{~cm}^{2} \mathrm{~V}^{-1} \mathrm{~s}^{-1}$, which was ascribed to the extended molecular orbital overlap (Figure 9d). ${ }^{[55]}$

In addition to widely exploited flanked and fused isoindigos, nitrogen atoms have also been introduced to modify the isoindigo backbones. ${ }^{[182]} \mathrm{N}$ atom of pyridine functions as an electron-accepting unit owing to its electronegative property. Huang et al. exploited computational screening to predict that the azaisoindigo with $7,7^{\prime}$-substitution was the best monomer (Figure 10a). ${ }^{[183]}$ This derivative displayed augmenting electrondeficient characteristics, minimized dihedral angles, better coplanarity and hence elevated charge transport, which resulted in ambipolar behavior $\left(2.33 / 0.78 \mathrm{~cm}^{2} \mathrm{~V}^{-1} \mathrm{~s}^{-1}\right.$ for holes/electrons) with bottom-contact/top-gate structure under air atmosphere (Figure 10b).

Meanwhile, incorporating fluorine into polymeric backbone has been recognized as a useful approach for high-mobility ambipolar carrier injection. ${ }^{[184]}$ Fluorine not only presents largest Pauling electronegativity of 4.0 (bring down the frontier molecular orbital energy levels) but also has extremely small van der Waals radius of $1.35 \AA$ (reduced steric hindrance and enhanced planarity). Moreover, the noncovalent molecular interactions of $\mathrm{F}$ atoms may facilitate the polymeric organization and crystallization capability. Gao et al. demonstrated multifluorinated conjugated copolymers by including $\mathrm{F}$ atoms into both donor ((E)-1,2-bis(3,4-difluorothien-2-yl)ethane) and acceptor (fluorinated isoindigo) moieties and observed notably high hole and electron mobilities up to 3.94 and $3.50 \mathrm{~cm}^{2} \mathrm{~V}^{-1} \mathrm{~s}^{-1}$, respectively (Figure 10c). ${ }^{[185]}$ Very recently, the largest mobility 


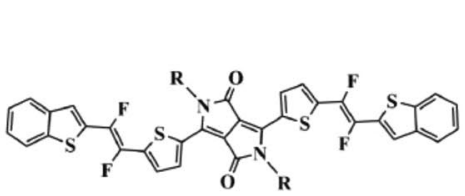

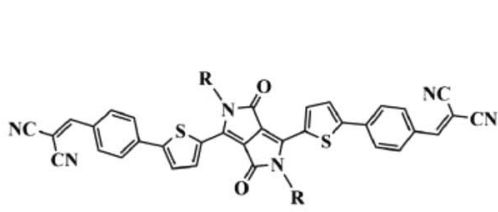

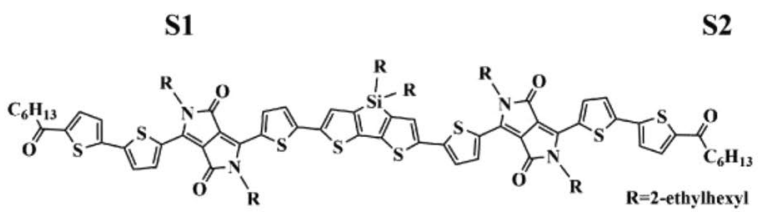

S2

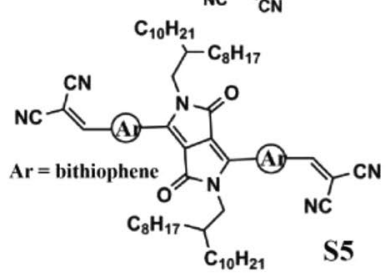
$\mathrm{C}_{8} \mathrm{H}_{17}$<smiles>[Mg][Mg]</smiles>

S3

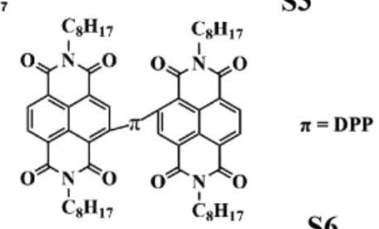

S4

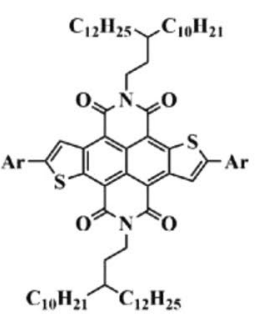

S7

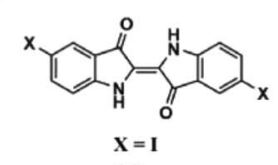

S8

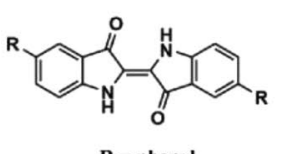

S9

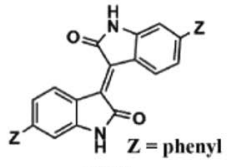

S10

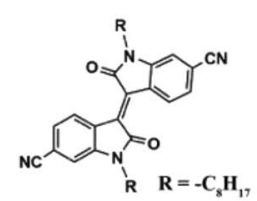

S11

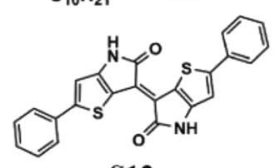

S12

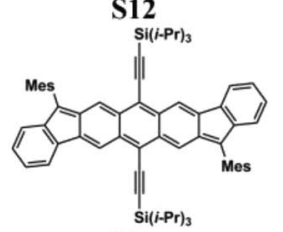

S13

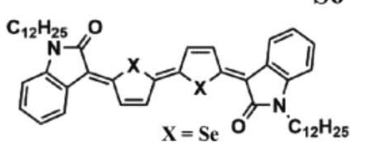

S14

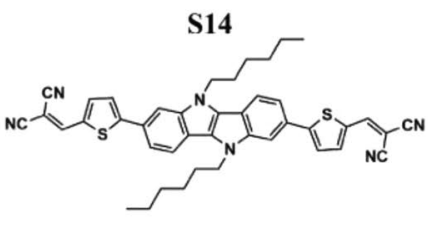

S15

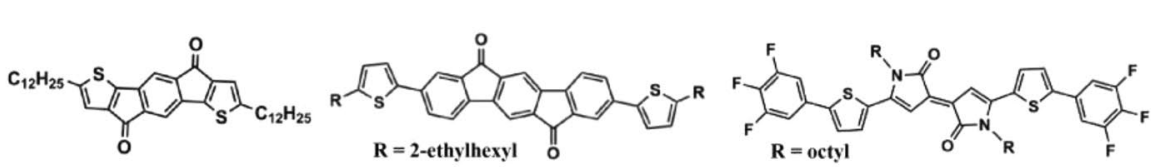

S16
S17
S18

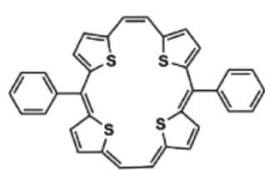

S20

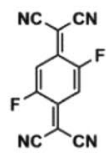

S21

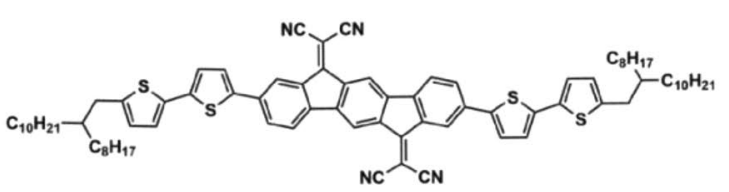

S19

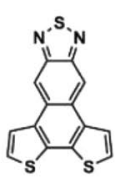

S22

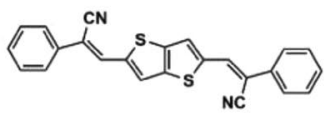

S23

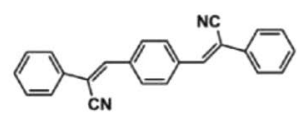

S24

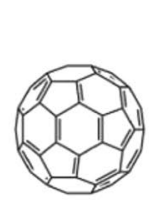

S25

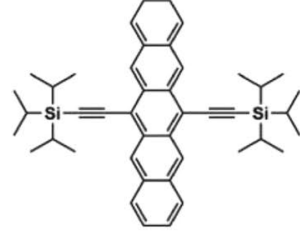

S26

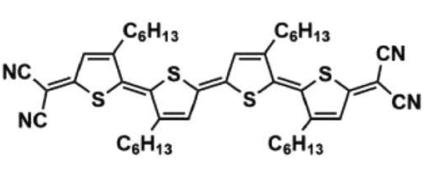

S27

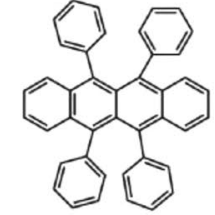

S28

Figure 8. Chemical structures of recent selected semiconducting organic small molecules and single crystals.

values $\left(\mu_{\mathrm{h}} / \mu_{\mathrm{e}}=\left(6.41 \mathrm{~cm}^{2} \mathrm{~V}^{-1} \mathrm{~s}^{-1}\right) /\left(6.76 \mathrm{~cm}^{2} \mathrm{~V}^{-1} \mathrm{~s}^{-1}\right)\right)$ of wellbalanced ambipolar isoindigo-based copolymers were achieved by Yang et al. (Figure 10d). ${ }^{[186]}$ The authors united fluorinated isoindigo with 3,3'-difluoro-2,2'-bithiophene and induced reduced energy levels, better coplanar architecture and crystallinity. They also brought in a concept of "effective mass" and found that the copolymers with smaller effective masses were favorable for better intramolecular charge transport (Figure 10e).

DPPs with planar architecture, intense $\pi-\pi$ interaction and strong aggregating properties have been studied a lot to realize ambipolar charge injection with superior mobilities (over amorphous-Si TFTs with mobility of $1 \mathrm{~cm}^{2} \mathrm{~V}^{-1} \mathrm{~s}^{-1}$ ) after the first demonstration of 3,6-diphenyl-DPP in 1974. ${ }^{[34,187-195]}$ Głowacki et al. explored three typical H-bonded DPP pigments with ambipolar charge mobilities $\left(0.01-0.06 \mathrm{~cm}^{2} \mathrm{~V}^{-1} \mathrm{~s}^{-1}\right)$ resulting from $\pi-\pi$ stacking generated by $\mathrm{H}$-bonded crystal. ${ }^{[196]}$

Researchers usually flank DPP core unit with two fivemembered heteroaryl groups (thiophene for DBT, furan for DBF, selenophene for DBS, thieno[3,2-b]thiophene for DBTT or thiazole-flanked DPP) due to their favorable properties such as coplanar backbone and intense intermolecular $\pi-\pi$ 
(a)

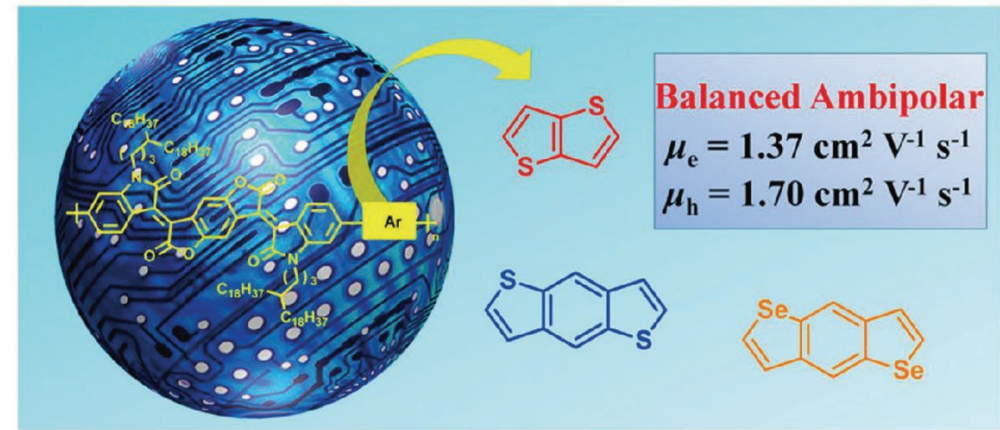

(b)
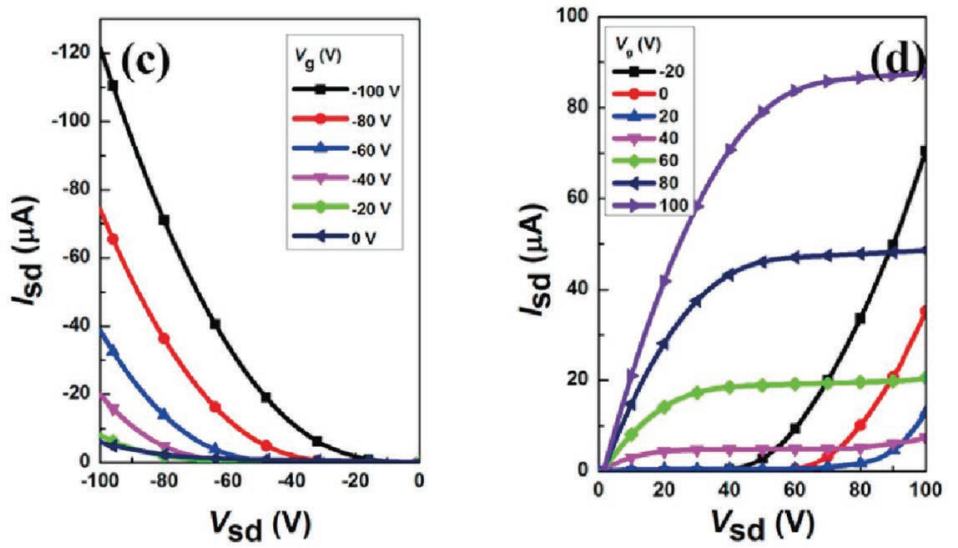

(c)
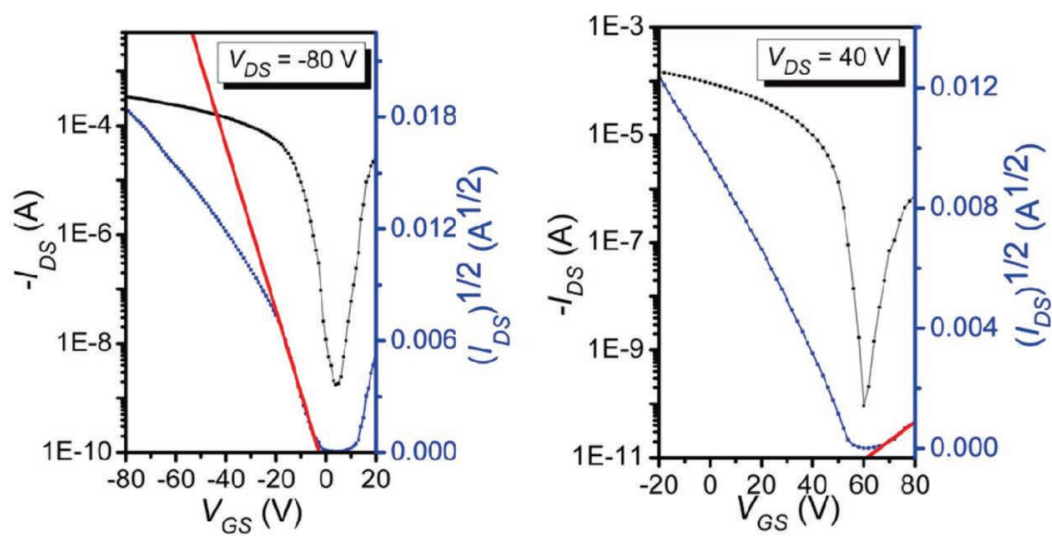

(d)

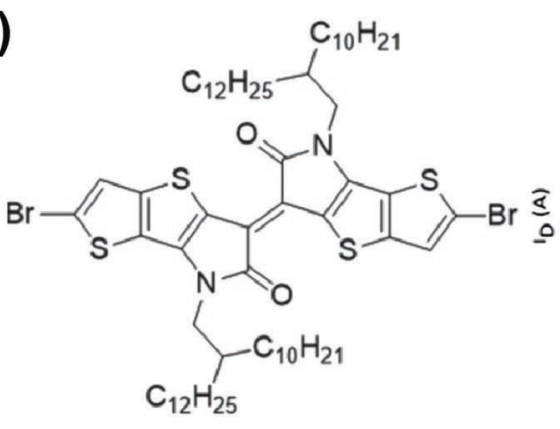

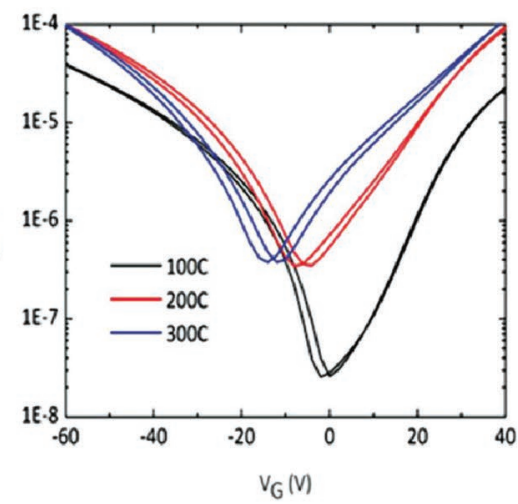

Figure 9. a) Molecular structures including BDOPV acceptor and different donors. b) Output curves of BDOPV-TT. Reproduced with permission. ${ }^{[178]}$ Copyright 2015, American Chemical Society. c) Transfer characteristics of bottom-gate top-contact OTFTs on the basis of conjugated polymers comprising DIID acceptor and $(E)-1,2$-bis (thiophen-2-yl)ethene donor (annealing conditions: temperature of $200^{\circ} \mathrm{C}$ for 10 minutes). Reproduced with permission. [179] Copyright 2016, American Chemical Society. d) Molecular structure of novel dibrominated thieno[3,2-b]thiophene isoindigo-based monomer unit; transfer curves of top-gate bottom-contact OFETs based on polymers consisting of thieno[3,2-b]thiophene isoindigo acceptor moiety and benzothiadiazole donor unit (various annealing temperatures: 100,200 , and $300{ }^{\circ} \mathrm{C} ; V_{\mathrm{D}}$ : $-60 \mathrm{~V}$ ). Reproduced with permission. ${ }^{[55]}$ Copyright $2014, \mathrm{Wiley}-\mathrm{VCH}$. 
(a)
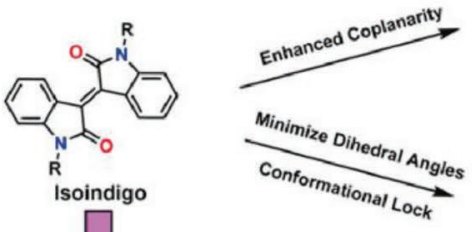

$\square$

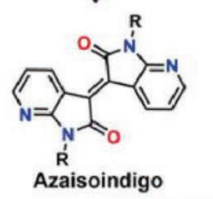

TGBC

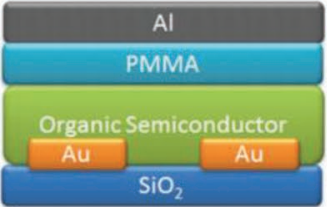

(b)

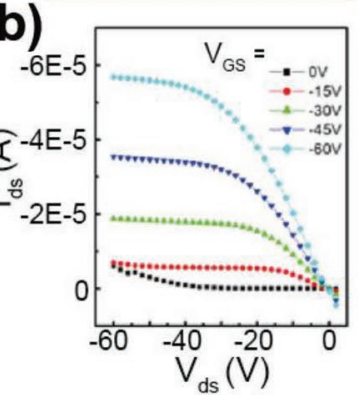

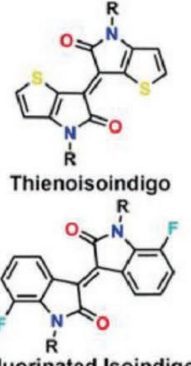

i (c)
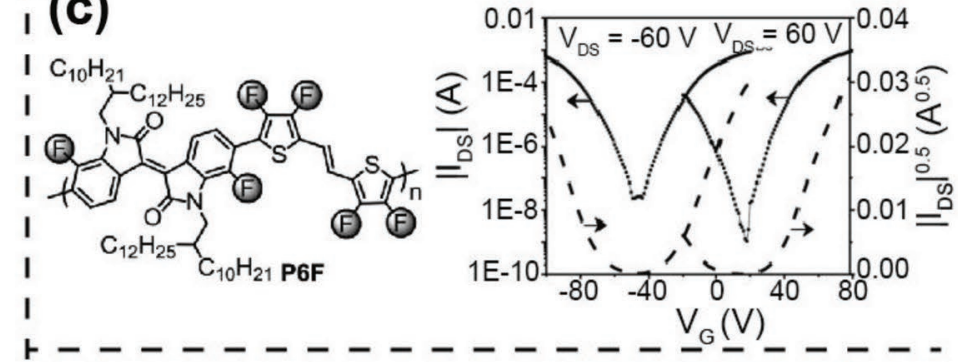

(d)

Minimized Dihedral Angles Between comonomers

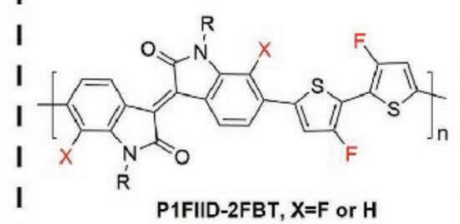

1

(e)

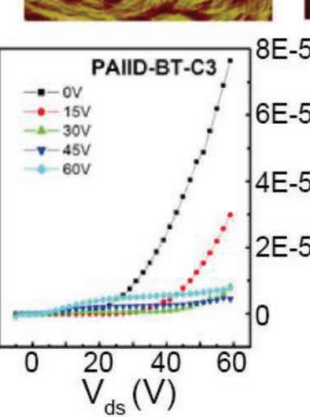

1

I
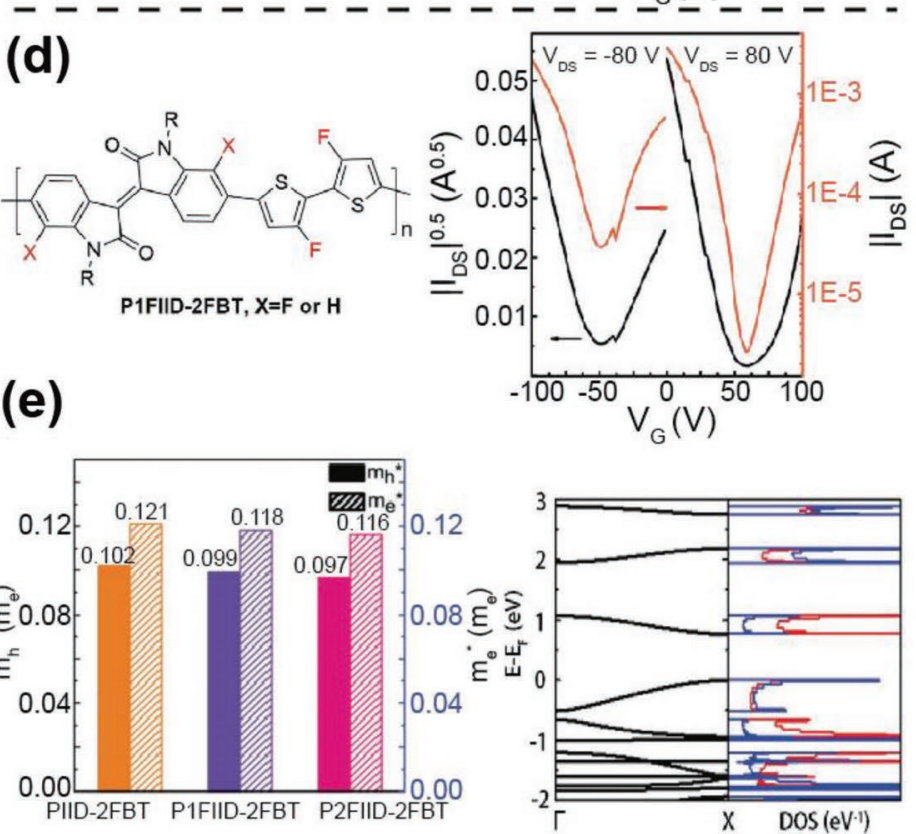

0.12

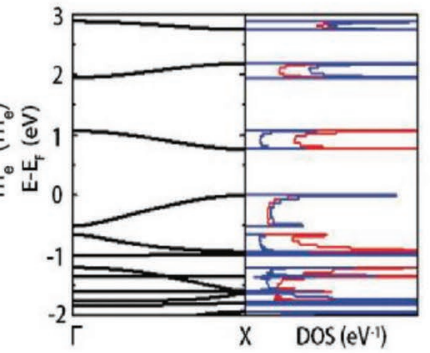

Figure 10. a) Molecular structures and respective advantages of different isoindigo derivatives; device configuration of top-gate bottom-contact OFETs; AFM morphology of PAIID-BT-C3 layers after annealing. b) Output curves of top-gate bottom-contact OFETs on the basis of PAIID-BT-C3 (measured at air atmosphere). Reproduced with permission. ${ }^{[183]}$ Copyright 2016, American Chemical Society. c) Molecular structure of conjugated polymer P6F and typical transfer curves of top-gate bottom-contact OFETs on the basis of P6F. Reproduced with permission. ${ }^{185}$ ] Copyright 2017, Wiley-VCH. d) Molecular structure of polymer P1FIID-2FBT and representative transfer curves of top-gate bottom-contact OFETs on the basis of P1FIID-2FBT. e) Effective masses of both two types of charge carriers according to theoretical calculations; band architectures as well as partial densities of states of P1FIID-2FBT (Blue and red lines indicate the contributions of donors and acceptors, respectively). Reproduced with permission. ${ }^{186]}$ Copyright 2017, Wiley-VCH.

interactions. ${ }^{[197-200]}$ Specifically, DBT is more promising for ambipolar performance on account of its planar structure and simple chemical modifications. ${ }^{[201-204]}$ Guo et al. incorporated two DPP isomers into one copolymer backbone and obtained balanced ambipolar carrier mobilities $\left(0.02 \mathrm{~cm}^{2} \mathrm{~V}^{-1} \mathrm{~s}^{-1}\right)$ by reason of highly ordered and extremely close packing distance of the active layer. ${ }^{[205]}$ Yang et al. first reported the novel acceptors, 2DPP (homocoupling of two DPPs) and fabricated flexible transistors on PET substrate with copolymers involving $2 \mathrm{DPP}$ as an accepting unit and thieno-[3,2-b]thiophene as a donating unit, which displayed high ambipolar mobilities of flexible polymer-based ambipolar transistors (4.16 and $3.01 \mathrm{~cm}^{2} \mathrm{~V}^{-1} \mathrm{~s}^{-1}$ for holes and electrons). ${ }^{[206]}$ Balanced charge mobilities exceeding $2 \mathrm{~cm}^{2} \mathrm{~V}^{-1} \mathrm{~s}^{-1}$ were extracted from DPPbased polymers with benzotriazole (BTZ) as electron-deficient moieties (Figure 11a). ${ }^{[207]}$ It was discovered that the introduction of linear alkyl side chain into DPP core could enhance the charge transport. Ambipolar behavior with very large hole mobility of $4.72 \mathrm{~cm}^{2} \mathrm{~V}^{-1} \mathrm{~s}^{-1}$ and electron mobility of $5.54 \mathrm{~cm}^{2} \mathrm{~V}^{-1} \mathrm{~s}^{-1}$ was observed in novel terpolymers (named PDPP2T-Se-Th) containing DPP core conjugated with thiophene and selenophene moieties (Figure 11b). ${ }^{[208]}$ It was found that the augment of the selenophene species in the copolymers resulted in increasing crystallization temperatures, terpolymer crystallinity and thereby the carrier mobility values. Xiao et al. reported the nanowire transistors on the basis of polymers composed of DPP cores, benzodithiophene units and thiazole linkers and demonstrated remarkably high well-balanced charge mobilities of 5.47 and $5.33 \mathrm{~cm}^{2} \mathrm{~V}^{-1} \mathrm{~s}^{-1}$ for holes and electrons, respectively (Figure 11c). ${ }^{[209]}$

Unsaturated linkages such as vinylene, acetylene and cyano units (i.e., cyanovinylene) are broadly utilized in DPPbased polymers because they can generate coplanar structure, strong $\pi$-conjugation and intense intermolecular $\pi-\pi$ stacking. Novel strong acceptors with enhanced planarity, vinylene- and acetylene-linked bis-benzothiadiazole (VBBT and 
(a)
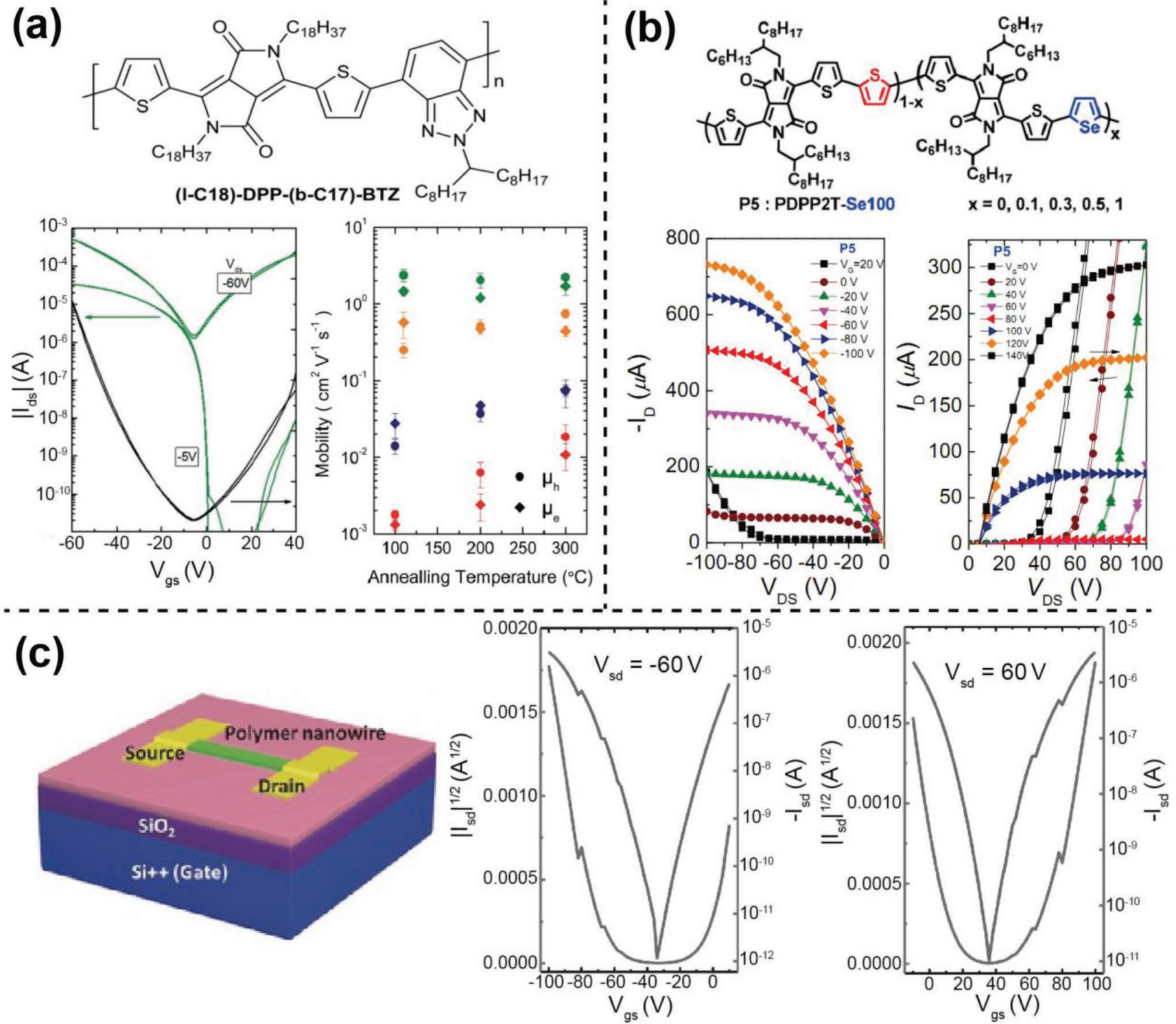

Figure 11. a) Chemical structure of DPP-benzotriazole copolymer $\left(\left(I-C_{18}\right)-D P P-\left(b-C_{17}\right)-B T Z\right)$; representative transfer curves of transistors (length is $20 \mu \mathrm{m}$ whereas width is $1000 \mu \mathrm{m})$ on the basis of $\left(\mathrm{I}-\mathrm{C}_{18}\right)$-DPP- $\left(\mathrm{b}-\mathrm{C}_{17}\right)$-BTZ thin films (annealing temperature: $110^{\circ} \mathrm{C}$ ); the relation between electron and hole mobilities of $\left(\mathrm{I}-\mathrm{C}_{18}\right)$-DPP-(b-C $\left.\mathrm{C}_{17}\right)-\mathrm{BTZ}$ (green) and various annealing temperatures. Reproduced with permission. ${ }^{[207]}$ Copyright 2015 , the Royal Society of Chemistry. b) Molecular structures of DPP-based polymers; typical output curves of OFETs based on P5 layers under p-channel (left) and n-channel (right) operations (fabrication method and condition: solution shearing; annealing temperature of $200{ }^{\circ} \mathrm{C}$ ). Reproduced with permission. ${ }^{[208]}$ Copyright 2014, American Chemical Society. c) Schematic structure of bottom-gate top-contact ambipolar transistors based on PDPP2Tz-BDT nanowires; transfer characteristics of OFETs on the basis of PDPP2Tz-BDT nanowires. Reproduced with permission. ${ }^{[209]} \mathrm{Copyright} 2015$, Wiley-VCH.

ABBT) were synthesized by Kim et al. and relatively balanced charge mobilities were achieved by combining VBBT and DPP (Figure 12a-c). ${ }^{[210]}$ Yun et al. reported conformation-insensitive copolymers composed of dithienyl-diketopyrrolopyrrole (tDPP) and acetylene-linked thiophene units and acquired good electron $\left(0.38 \mathrm{~cm}^{2} \mathrm{~V}^{-1} \mathrm{~s}^{-1}\right)$ and hole $\left(2.19 \mathrm{~cm}^{2} \mathrm{~V}^{-1} \mathrm{~s}^{-1}\right)$ transport performance because of the extended delocalization of the electrons induced by sp-hybridized carbons of acetylene groups (Figure 12d). ${ }^{[211]}$ Later, by introducing fluorine atoms to the $\beta$-positions of vinylene-linked thiophene units, Gao et al. demonstrated the synthesis of poly[2,5-bis(2-decyltetradecyl)
pyrrolo[3,4-c]pyrrole-1,4(2H,5H)-dione- alt-5,5'-di(thiophen-2yl)-2,2'-(E)-1,2-bis(3,4-difluorothien-2-yl)ethene] (PDPP-4FTVT) by direct arylation polycondensation and obtained increased balanced ambipolar carrier mobilities as high as 3.40 and $5.86 \mathrm{~cm}^{2} \mathrm{~V}^{-1} \mathrm{~s}^{-1}$ for holes and electrons, respectively (Figure 12e)..$^{[212]}$ In order to tune charge transport of conjugated polymers, Khim et al. copolymerized two large building blocks (poly[2,5-bis(2-octyldodecyl)pyrrolo[3,4-c]pyrrole-1,4 $(2 H, 5 H)$-dione- $(E)$-[2,2-bithiophen]-5-yl)-3-(thiophen2-yl)acrylonitrile] (DPP-CNTVT) as n-type semiconductor and DPP-selenophene-vinylene-selenophene (DPP-SVS) as p-type 
(a)

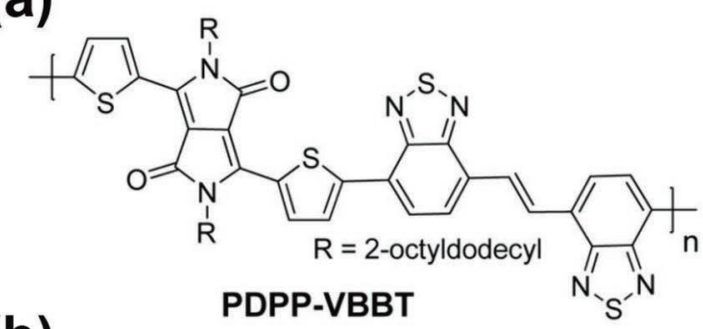

(b)

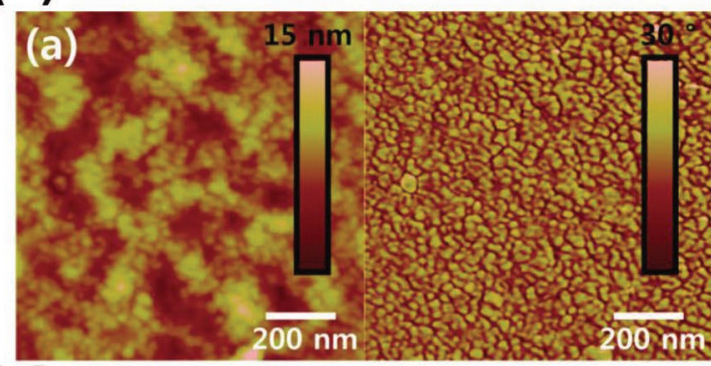

(c)
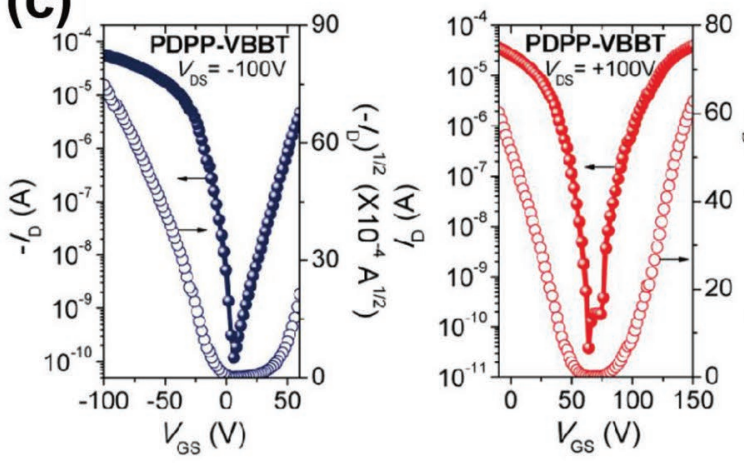

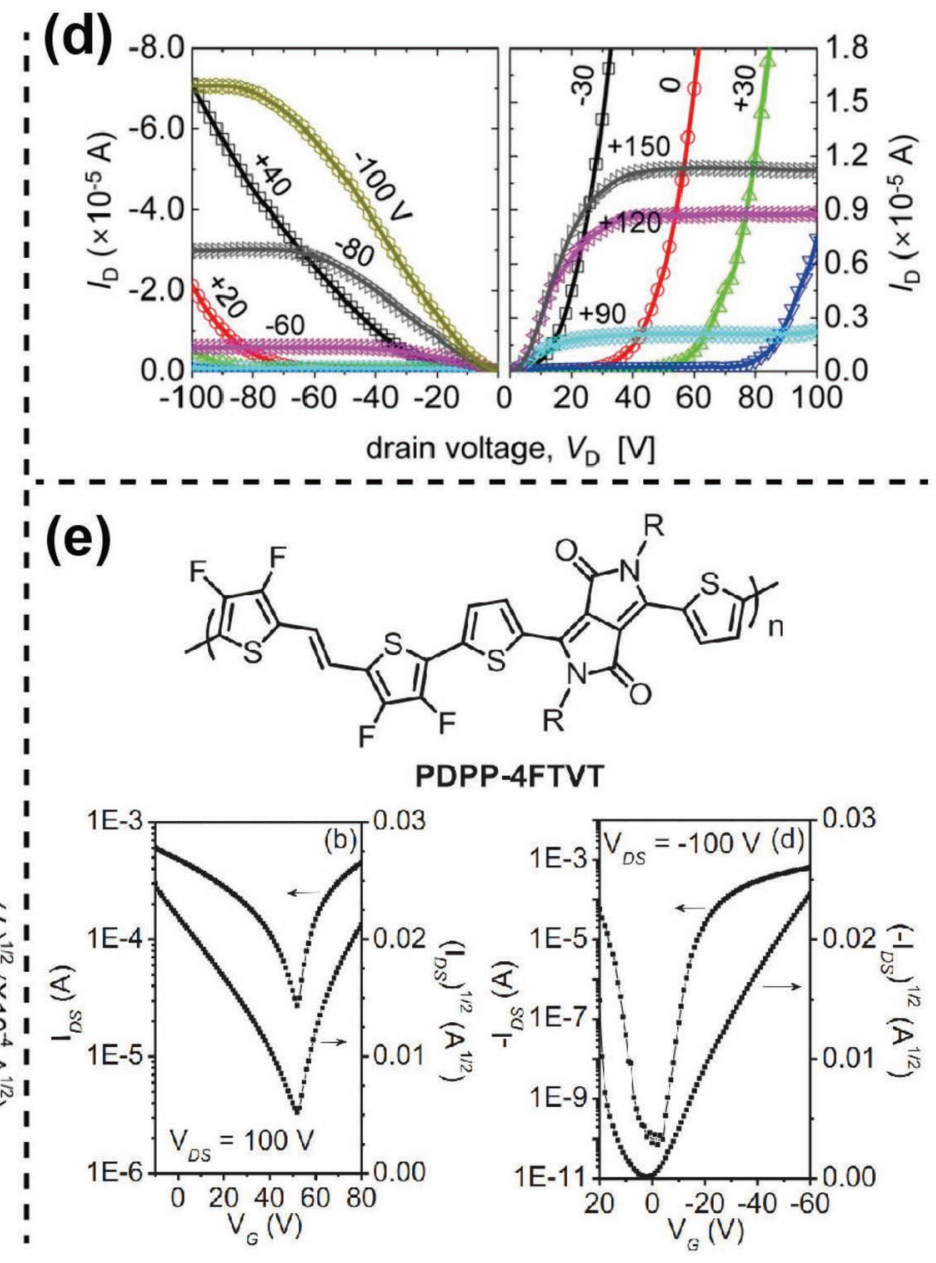

Figure 12. a) Molecular structure of conjugated polymer combining VBBT and DPP (named PDPP-VBBT). b) AFM height profile (left) and phase information (right) of PDPP-VBBT thin film prepared by drop-casting method (annealing temperature: $220^{\circ} \mathrm{C}$ ). C) Typical transfer curves of optimized OFETs based on PDPP-VBBT. Reproduced with permission. ${ }^{[210]}$ Copyright 2014, American Chemical Society. d) Output characteristics of OFETs based on copolymers consisting of dithienyl-diketopyrrolopyrrole and acetylene-linked thiophene units (named PDPP-TAT). Reproduced with permission. ${ }^{211]}$ Copyright 2014, American Chemical Society. e) Chemical structure of synthesized PDPP-4FTVT; representative transfer curves of transistors on the basis of PDPP-4FTVT operated in n-channel (left) and p-channel (right) modes (annealing conditions: temperature of $200^{\circ} \mathrm{C}$ and duration of 10 min). Reproduced with permission. ${ }^{[212]}$ Copyright 2015, Wiley-VCH.

semiconductor) and realized transition from hole transport to electron transport by modulating various copolymerization ratios (Figure 13a). ${ }^{[213]}$ It was found that the identical backbone (DPP) existed in the two semiconductors gave rise to highly coplanar architecture and superior packing motif, which led to well-balanced mobility values at equal fraction $\left(\mu_{\mathrm{h}} / \mu_{\mathrm{e}}=\left(3.35 \mathrm{~cm}^{2} \mathrm{~V}^{-1} \mathrm{~s}^{-1}\right) /\left(3.18 \mathrm{~cm}^{2} \mathrm{~V}^{-1} \mathrm{~s}^{-1}\right)\right)$ (Figure 13b,c). Cyano unit with intense electron withdrawing property and intermolecular interaction also received comprehensive attention. Park et al. inserted a cyanovinylene group between diketopyrrolo[3,4-c]pyrrole and benzo[1,2-b:4,5- $\left.b^{\prime}\right]$ dithiophene (named PBCDC) and demonstrated balanced ambipolar behavior with high thermal stability, showing no degradation with the annealing temperature $\left(80-250{ }^{\circ} \mathrm{C}\right)$ (Figure 13d). ${ }^{[214]}$

Recently, in addition to aforementioned five-membered ringflanked DPP-based copolymers, Sun et al. first utilized two sixmembered rings, 2-pyridinyl to flank DPP core unit, namely, DBPy (Figure 13e).[215] The 2-pyridinyl substituents were beneficial for lower LUMO level, meanwhile showed relatively small steric effects and induced increased coplanarity. The fabricated transistors based on conjugated polymers consisting of DBPy and bithiophene (named PDBPyBT) exhibited large hole and electron mobilities of 2.78 and $6.30 \mathrm{~cm}^{2} \mathrm{~V}^{-1} \mathrm{~s}^{-1}$, which was ascribed to better crystallinity and closer $\pi-\pi$ stacking distance $(0.36 \mathrm{~nm})$ of the polymers (Figure 13f). DBPy was demonstrated as a promising new building block of conjugated semiconducting materials. ${ }^{[216,217]}$ Lee et al. researched a battery of D-A polymers (named PTDPPSe-Si) composed of electron-deficient DPP core with siloxane-solubilizing groups and electronrich selenophene (Figure 14a). ${ }^{[218]}$ By regulating the alkyl spacer length, it was found that the copolymers modified with pentyl spacers displayed unprecedentedly large charge mobility values (8.84 $\mathrm{cm}^{2} \mathrm{~V}^{-1} \mathrm{~s}^{-1}$ for holes and $4.34 \mathrm{~cm}^{2} \mathrm{~V}^{-1} \mathrm{~s}^{-1}$ for electrons).

Very recently, Ni et al. put forward a two-step C-H-activated polymerization approach to synthesize conjugated copolymers consisting of DPP-benzothiadiazole-DPP (DBD) moieties and 

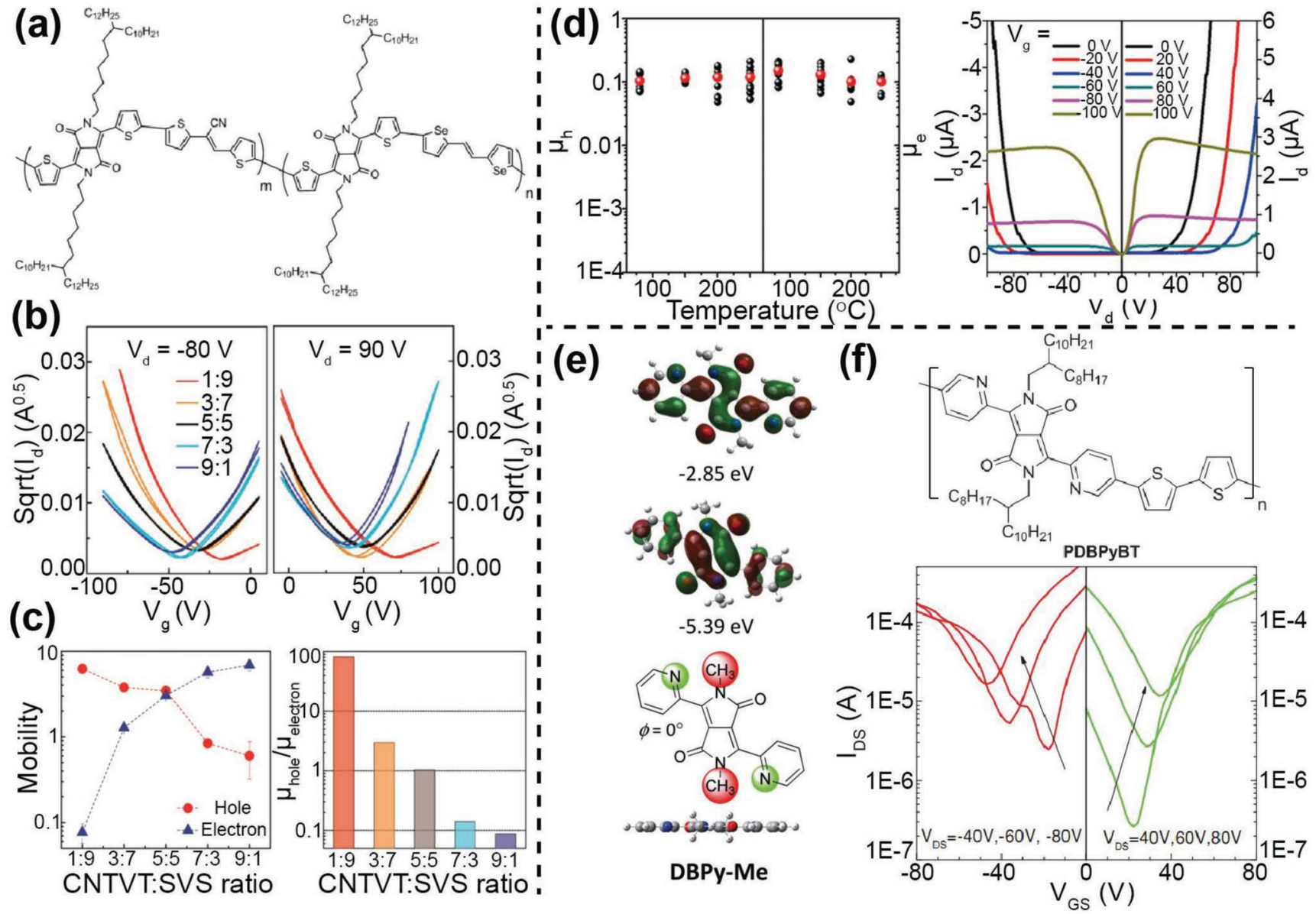

Figure 13. a) Polymeric structure of copolymer DPP-CNTVT (acceptor):DPP-SVS (donor). b) Representative transfer curves (left: p-channel; right: n-channel) of top-gate bottom-contact OFETs (PMMA dielectrics) on the basis of copolymer DPP-CNTVT:DPP-SVS with five various copolymerization ratios (1:9, 3:7, 5:5, 7:3, and 9:1). c) The dependence of charge mobilities (left) and polarity balance (definition: $\mu_{\text {hole }} / \mu_{\text {electron; }}$ right image) on different copolymerization ratios between aforementioned two moieties. Reproduced with permission. ${ }^{[213]}$ Copyright 2016, American Chemical Society. d) Relation between hole and electron mobilities of polymer PBCDC and various annealing temperatures (ten transistors were measured and the red spheres represented average carrier mobilities); typical output curves of PBCDC. Reproduced with permission. ${ }^{[214]}$ Copyright 2016, American Chemical Society. e) The electron distribution graphs of HOMO and LUMO as well as the geometry of 2,5-dimethyl-3,6-di(pyridin-2-yl) pyrrolo[3,4-c]pyrrole-1,4 $(2 \mathrm{H}, 5 \mathrm{H}$ )dione (DBPy-Me) acquired via density functional theory (DFT) calculations. f) Chemical structure of conjugated polymer PDBPyBT and representative transfer characteristics of top-gate bottom-contact PDBPyBT-based OFETs with channel length and width of $30 \mu \mathrm{m}$ and $1 \mathrm{~mm}$, respectively. The annealing temperature was set at $100^{\circ} \mathrm{C}$. Reproduced with permission. ${ }^{[215]}$ Copyright 2014 , Wiley- $\mathrm{VCH}$.

thiophene (named PDBD-T)/selenophene (named PDBD-Se) groups (Figure 14b). ${ }^{[12]}$ It was found that spin-coated top-gate bottom-contact PDBD-Se-based flexible organic field-effect transistors (OFETs) exhibited superior ambipolar performance with record large charge mobilities of 8.90 and $7.71 \mathrm{~cm}^{2} \mathrm{~V}^{-1} \mathrm{~s}^{-1}$ for holes and electrons, respectively (Figure 14c).

Except for isoindigo and DPP, pyridal[2,1,3]thiadiazole (PT) with electron-withdrawing property and coplanar backbone is also a good acceptor and applied for constructing D-A copolymers. Very recently, Zhu et al. designed the first regioregular ambipolar copolymer comprising PT units (named PBPTV) (Figure 14d). ${ }^{[25]}$ By connecting two PT acceptors, the authors utilized novel bispyridal[2,1,3]thiadiazole (BPT) group as electron-deficient units and (E)-2-(2-(thiophen-2-yl)vinyl)-thiophene (TVT) group as electron-rich units and realized lower LUMOs and enhanced interchain organization, which finally led to the record high balanced carrier mobilities $\left(6.87\right.$ and $8.49 \mathrm{~cm}^{2} \mathrm{~V}^{-1} \mathrm{~s}^{-1}$ for holes and electrons) (Figure 14e). This copolymer together with the above mentioned PDBD-Se are the best ambipolar D-A copolymers and present the highest mobilities to date. More success of D-A copolymers with dramatically high carrier mobilities is expected.

\subsubsection{Polymer Composites}

The ambipolar concept of hybrid heterojunction film derived from codeposited solution processable binary polymer blends is attractive for achieving large-area integrated circuits on account of simple fabrication procedures. ${ }^{[219-222]}$ Nevertheless, it is difficult to control the microstructure and surface arrangement of the two semiconductors and hence the morphology of semiconducting thin film, which is crucial for balanced carrier transfer. Recently, relatively few bipolar binary blends are reported. Gemayel et al. demonstrated enhanced hole transport 
(a)

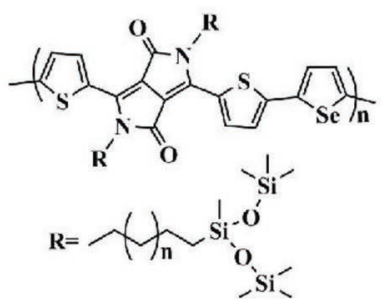
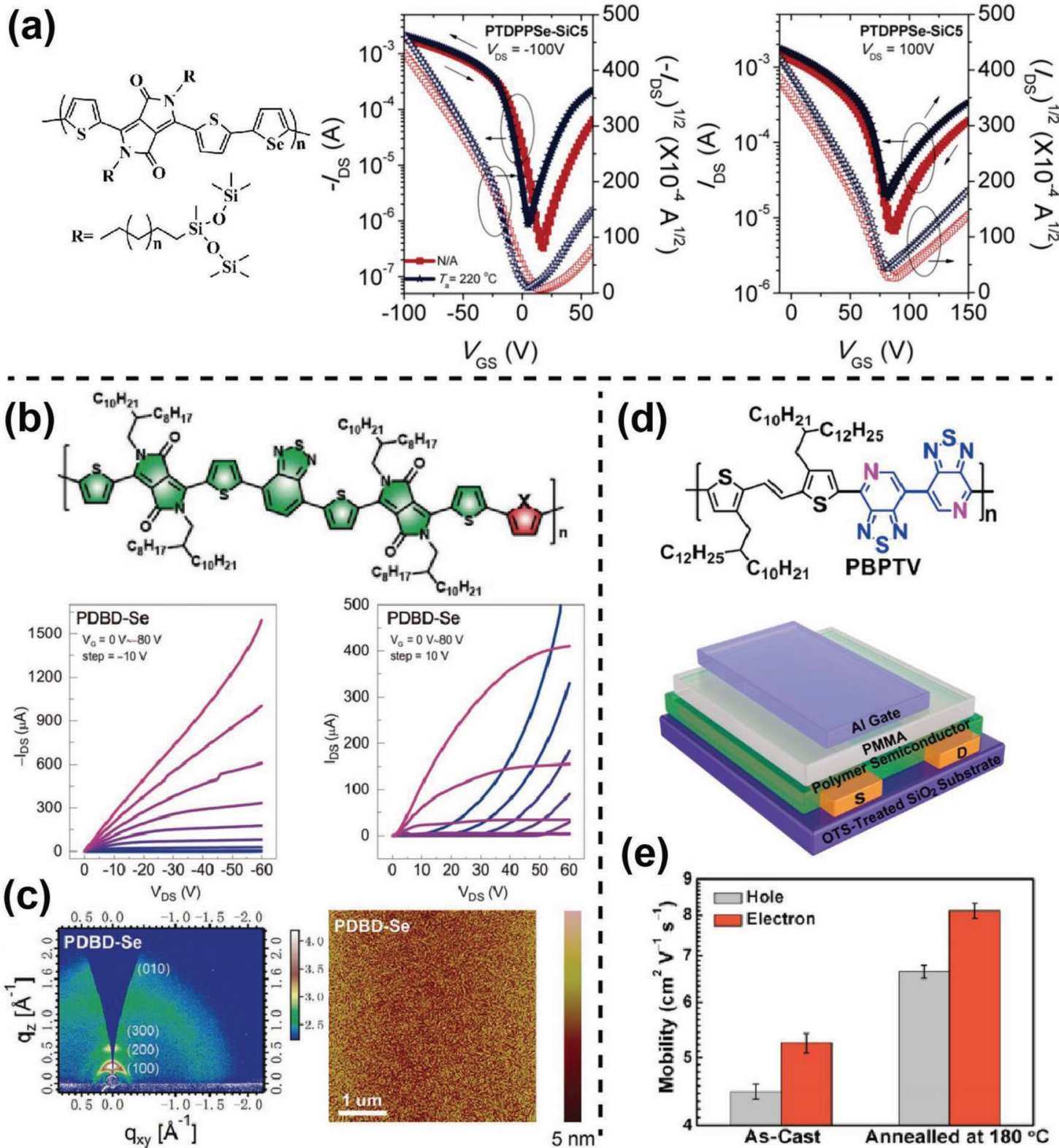

Figure 14. a) Chemical structure of polymer PTDPPSe-Si and transfer curves of OFETs based on solution-shearing PTDPPSe-SiC5 thin films (annealing temperature: $220^{\circ} \mathrm{C}$ ) operated under hole- (left) and electron-domination (right) modes, respectively. Reproduced with permission. ${ }^{[218]}$ Copyright 2013 , American Chemical Society. b) Polymeric structure of PDBD-T and PDBD-Se and typical output curves of OFETs on the basis of spin-coated PDBD-Se films (left: p-type; right: $n$-type). c) Grazing-incidence wide-angle x-ray scattering (GIWAXS) pattern (left) and AFM morphology (right) of PDBD-Se. Reproduced with permission. ${ }^{[12]}$ Copyright 2019, Wiley-VCH. d) Chemical structure of regioregular copolymer PBPTV and schematic diagram of topgate bottom-contact OFETs. e) Statistical electron and hole mobilities obtained from 30 OFETs. Reproduced with permission. ${ }^{[25]}$ Copyright 2017 , American Chemical Society.

for bipolar behavior by codepositing solution-processing liquid-phase exfoliated graphene (in $o$-DCB) and electron-deficient dominant poly $[N, N$-9-bis(2-octyldodecyl)-naphthalene1,4,5,8-bis(dicarboximide)-2,6-diyl]-alt-5,59-(2,29-bithiophene)] (P(NDI2OD-T2)) due to well-embedded graphene sheets inside the polymeric network. ${ }^{[223]}$ Kang et al. proposed a single-step solution-processable organic semiconducting layer via utilizing the mixed solution of ambipolar quinoidal biselenophene and insulating poly(2-vinylnaphthalene) (PVN) binder. ${ }^{[74]}$
Microanalysis based on secondary ion mass spectrometry and AFM manifested QBS molecules were uniformly phase-separated and located at the bottom of the polymeric matrix and formed homogenous film morphology, which gave rise to four times larger charge mobilities compared with single QBS-based transistors. Controlling the phase separation of the semiconducting materials ( $\mathrm{p}$ - or n-type) can generate individual and consecutive migration paths for holes/electrons and thereby result in well controlled ambipolar performance. Janasz et al. 
put forward a novel preaggregation concept by aggregating electron-donating poly(3-hexylthiophene) (P3HT) in advance of the solution blend process with electron-accepting phenyl$\mathrm{C}_{61}$-butyric acid methyl ester (PCBM). ${ }^{[24]}$ The authors discovered that the fibrillar P3HT aggregates were well dispersed in PCBM matrix and good phase separation was realized owing to the immiscibility between P3HT aggregates and PCBM network, which finally led to prominently increased efficient bipolar mobilities of $0.01 \mathrm{~cm}^{2} \mathrm{~V}^{-1} \mathrm{~s}^{-1}$. By changing PCBM with $\mathrm{P}$ (NDI2OD-T2), Yan et al. demonstrated well-balanced ambipolar behavior of OFETs on the basis of P(NDI2OD-T2)/P3HT blends with trichloro(octyl)silane (OTS)-modified blocking dielectric. ${ }^{[225]}$ It was found that the ambipolar mobilities were comparable to that in unipolar transistors, which could be explained from the polymer networks (provide the transporting pathways of holes and electrons) in the AFM morphology.

The energy levels, electrical performance, processing methods as well as device structures of recent selected polymer materials are summarized in Table 2 and the polymeric structures of them are listed in Figure 15. Apparently, ambipolar transistors on the basis of D-A polymers exhibit much higher charge mobilities (approach $9 \mathrm{~cm}^{2} \mathrm{~V}^{-1} \mathrm{~s}^{-1}$ ) compared to these based on organic small molecules and undoubtedly better ambipolar performance of this area can be expected with the further progress of polymeric engineering.

\subsection{Carbon Nanotubes}

Single-walled carbon nanotubes (SWNTs) with 1D architecture have received extensive scientific attention due to their remarkable charge mobilities and good mechanical flexible properties. ${ }^{[226-233]}$ SWNTs based on the structure of the rolled-up nonleaky graphene cylinder possess relatively small diameters of 1-2 nm, energy gaps of $0.7-1 \mathrm{eV}$ and intrinsic semiconducting characteristics (direct bandgap semiconductor), which is totally different from the metallic graphene (no bandgap). ${ }^{[234]}$ SWNTs also can be classified as metallic (about one-third) or semiconducting (around two-thirds) judged by their chiral vectors. Carbon nanotube was first proposed by lijima in 1991, ${ }^{[235,236]}$ after which SWNTs have been widely studied. However, it is challenging to grow and obtain SWNTs with specific structures for better performance among various carbon nanotubes.

Thus, in the beginning, researchers selected single-strand SWNT as the semiconducting layer of transistors and demonstrated extremely large charge mobilities $\left(\mu \approx 10^{5} \mathrm{~cm}^{2} \mathrm{~V}^{-1} \mathrm{~s}^{-1}\right)$ and on/off ratio $\left(10^{7}\right) \cdot{ }^{[237,238]}$ Although these electrical properties meet the demand for CMOS devices and integrated circuits, single-strand carbon nanotube is thought to be incompatible for mass production of transistors. Assembling individual nanotube precisely in nanometer scale, minimizing the performance variation of different devices together with complex fabrication procedures including CVD and lithography are difficulties for the further development of SWNT transistors.

Subsequently, SWNT networks were fabricated via various approaches and used as semiconductors in transistors. One alternative method is CVD, in which SWNTs are deposited onto the substrate surface with the existence of catalyst, carbon and hydrogen gas within the furnace. The CVD-grown SWNTs exhibit high charge mobilities $\left(50-200 \mathrm{~cm}^{2} \mathrm{~V}^{-1} \mathrm{~s}^{-1}\right)$. Nevertheless, as stated, the fabrication of metallic nanotubes is inevitable, which can induce larger current inside the channel due to superior charge transport and carrying capability of "metal" and therefore smaller on/off ratio (less than $10^{5}$ ) is achieved. This tradeoff together with high CVD working temperature is detrimental to device performance.

Another much easier technique to grow SWNT networks is solution process for instance spin coating, drop casting, inkjet/ aerosol printing, etc. ${ }^{[239-242]}$ During solution deposition, the prepared printable nanotube ink solution usually comprises various probable SWNTs and has relatively low purity. Thus, selectively removing the metallic nanotubes from mixed inks is indispensable to obtain semiconducting SWNTs with unitary chirality and controllable length. To date, scientists have separated diverse SWNTs effectively mainly through the noncovalent functionalization of nanotubes, which can maintain the electronic characteristics of carbon nanotubes. The representative sorting and purifying methods contain density gradient centrifugation (DGC), ${ }^{[243-245]}$ DNA-based separation, ${ }^{[246,247]}$ gel chromatography, ${ }^{[248]}$ electrophoresis, ${ }^{[249,250]}$ and polymer sorting ${ }^{[251,252]}$. DGC employs the buoyant variance of diverse SWNTs (dominate by their diameters) to generate a density gradient by blending original unseparated nanotubes and surfactants and then exploits ultracentrifugal forces to make metallic and semiconducting nanotubes apart. In spite of the realization of SWNTs sorting (including length, diameters and electronic types), fractional separated products are still mixtures and DGC owns its limitation. ${ }^{[244]}$ DNA-based separation is rarely reported and utilizes DNA sequences to selectively wrap the nanotubes to form DNA-SWNT hybrids to achieve the purification of single-chirality SWNTs in the light of chromatographic purification. ${ }^{[246,247]}$

Nevertheless, gel chromatography is a common technique for separation of biological and chemical polymers. The blended surfactants possess various affinity effects with metallic and semiconducting SWNTs and hence they will have distinct sizes after interaction. Thus, after passing the measured blended solution through a chromatographic column with certain gel pore size, larger SWNTs (wrapped by surfactants and larger than gel pores) will go through the gaps between gels directly and quickly. ${ }^{[24]}$ However, this approach is only suitable for SWNTs with small diameter range. Dielectrophoresis (DEP) exploits various motion phenomena of metallic and semiconducting nanotubes under a nonuniform electric field to sort them. The translational motion depends on different dielectric constants of SWNTs (about 1000 for metallic SWNTs and 5 for semiconducting SWNTs), electrical characteristics of surrounding medium and the frequency of the field strength change, etc. ${ }^{[249]}$ This strategy is effective but restricted on account of low yield.

Polymer sorting is more effective to purify the semiconducting tubes from their mixtures and has been proverbially employed since the report of Nish et al. in 2007. They put forward the first conjugated polymer poly(9,9-dioctylfluorenyl2,7-diyl) (named PFO) to selectively disperse different kinds of SWNTs. ${ }^{[252]}$ The separation principle is on the basis of the noncovalent interactions of SWNTs and polymers. During the 
Table 2. Summary of electrical properties, performance, processing methods, and device architectures of ambipolar transistors on the basis of semiconducting polymers.

\begin{tabular}{|c|c|c|c|c|c|c|c|}
\hline \multirow[t]{2}{*}{ Classification } & \multirow[t]{2}{*}{$\begin{array}{c}\text { Organic } \\
\text { semiconductors }\end{array}$} & \multirow[t]{2}{*}{$\begin{array}{c}\mathrm{HOMO} \\
{[\mathrm{eV}]}\end{array}$} & \multirow[t]{2}{*}{$\begin{array}{l}\text { LUMO } \\
{[\mathrm{eV}]}\end{array}$} & \multicolumn{2}{|c|}{$\begin{array}{l}\text { Mobilities } \\
{\left[\mathrm{cm}^{2} \mathrm{~V}^{-1} \mathrm{~s}^{-1}\right]}\end{array}$} & \multirow[t]{2}{*}{$\begin{array}{l}\text { Processing methods and } \\
\text { semiconductor configurations }\end{array}$} & \multirow[t]{2}{*}{ Ref. } \\
\hline & & & & $\mu_{\mathrm{e}}$ & $\mu_{\mathrm{h}}$ & & \\
\hline \multirow[t]{10}{*}{$\begin{array}{l}\text { Single-component polymers } \\
\text { Isoindigo-based polymers }\end{array}$} & P1 & -5.82 & -3.83 & 3.50 & 3.94 & $\begin{array}{l}\text { Spin coating } \\
\text { Single layer }\end{array}$ & [185] \\
\hline & P2 & -5.3 & -3.3 & 0.5 & 0.2 & $\begin{array}{l}\text { Spin coating } \\
\text { Single layer }\end{array}$ & [182] \\
\hline & P3 & -5.73 & -4.17 & 0.029 & 0.018 & $\begin{array}{l}\text { Spin coating } \\
\text { Single layer }\end{array}$ & [180] \\
\hline & P4 & -4.9 & -3.9 & 0.7 & 0.4 & $\begin{array}{l}\text { Spin coating } \\
\text { Single layer }\end{array}$ & [55] \\
\hline & P5 & -5.63 & -3.55 & 6.76 & 6.41 & $\begin{array}{l}\text { Spin coating } \\
\text { Single layer }\end{array}$ & [186] \\
\hline & P6 & -5.55 & -4.03 & 1.08 & 0.30 & $\begin{array}{l}\text { Spin coating } \\
\text { Single layer }\end{array}$ & [153] \\
\hline & P7 & -5.70 & -3.88 & 1.37 & 1.70 & $\begin{array}{l}\text { Spin coating } \\
\text { Single layer }\end{array}$ & [178] \\
\hline & P8 & -5.60 & -3.71 & 0.09 & 0.19 & $\begin{array}{l}\text { Spin coating } \\
\text { Single layer }\end{array}$ & [173] \\
\hline & P9 & -5.24 & -3.58 & 0.087 & 1.79 & $\begin{array}{l}\text { Spin casting } \\
\text { Single layer }\end{array}$ & [179] \\
\hline & P10 & -5.65 & -3.84 & 0.50 & 0.51 & $\begin{array}{l}\text { Spin coating } \\
\text { Single layer }\end{array}$ & [174] \\
\hline \multirow[t]{13}{*}{ DPP-based polymers } & P11 & -5.19 & -3.50 & 0.02 & 0.02 & $\begin{array}{l}\text { Drop casting } \\
\text { Single layer }\end{array}$ & [205] \\
\hline & P12 & - & -4.0 & 5.33 & 5.47 & $\begin{array}{l}\text { Drop casting } \\
\text { Nanowire }\end{array}$ & [209] \\
\hline & $\mathrm{P} 13$ & -5.15 & -3.90 & 1.5 & 2.4 & $\begin{array}{l}\text { Spin coating } \\
\text { Single layer }\end{array}$ & [207] \\
\hline & P14 & -5.37 & -3.54 & 3.01 & 4.16 & $\begin{array}{l}\text { Spin coating } \\
\text { Single layer }\end{array}$ & [206] \\
\hline & P15 & -5.36 & -3.50 & 5.86 & 3.40 & $\begin{array}{l}\text { Spin casting } \\
\text { Single layer }\end{array}$ & [212] \\
\hline & P16 & -5.34 & -3.43 & 0.13 & 0.32 & $\begin{array}{l}\text { Drop casting } \\
\text { Single layer }\end{array}$ & [210] \\
\hline & P17 & -5.15 & -3.48 & 5.54 & 4.72 & $\begin{array}{l}\text { Solution shearing } \\
\text { Single layer }\end{array}$ & [208] \\
\hline & P18 & -5.39 & -3.89 & 0.38 & 2.19 & $\begin{array}{l}\text { Spin coating } \\
\text { Single layer }\end{array}$ & [211] \\
\hline & P19 & -5.37 & -3.93 & 0.2 & 0.2 & $\begin{array}{l}\text { Spin coating } \\
\text { Single layer }\end{array}$ & [214] \\
\hline & P20 & -5.36 & -3.52 & 3.03 & 3.15 & $\begin{array}{l}\text { Spin coating } \\
\text { Single layer }\end{array}$ & [213] \\
\hline & P21 & -5.69 & -4.33 & 6.30 & 2.78 & $\begin{array}{l}\text { Spin coating } \\
\text { Single layer }\end{array}$ & [215] \\
\hline & P22 & -4.91 & -3.65 & 2.20 & 3.97 & $\begin{array}{l}\text { Solution shearing } \\
\text { Single layer }\end{array}$ & [34] \\
\hline & P23 & -5.10 & -3.49 & 4.34 & 8.84 & $\begin{array}{l}\text { Solution shearing } \\
\text { Single layer }\end{array}$ & [218] \\
\hline PT-based polymer & P24 & -5.61 & -3.66 & 8.49 & 6.87 & $\begin{array}{l}\text { Spin coating } \\
\text { Single layer }\end{array}$ & [25] \\
\hline
\end{tabular}




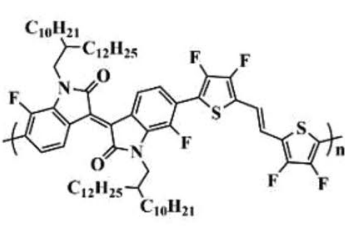

P1

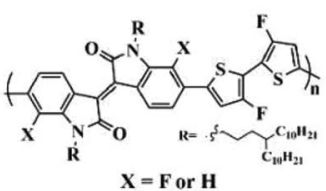

P5

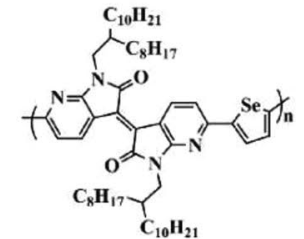

P2

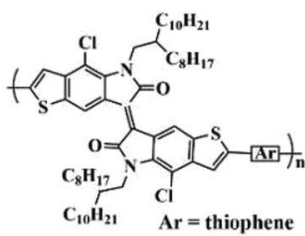

P3

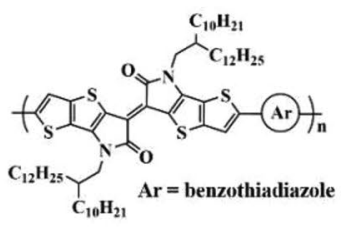

P4

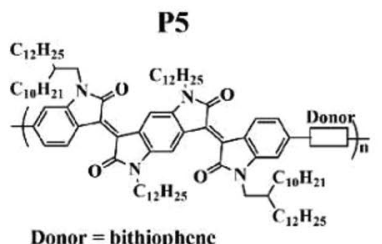

P8

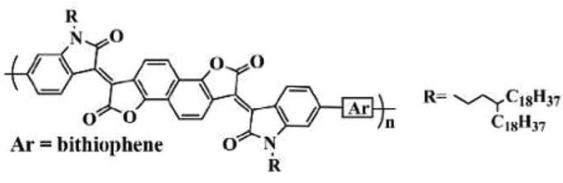

P10

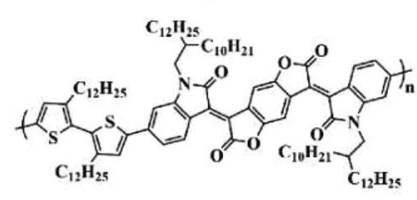

P6

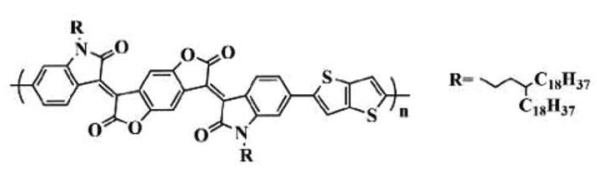

P7

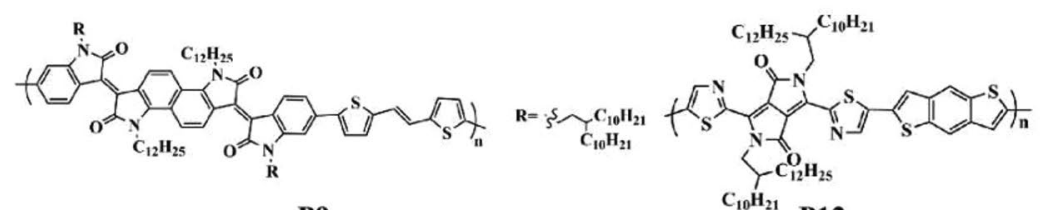

P12

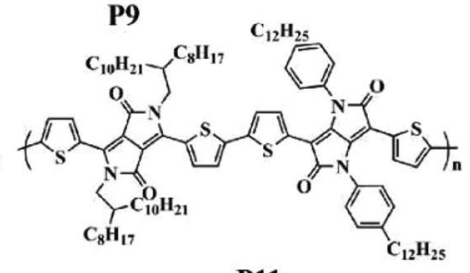

P11

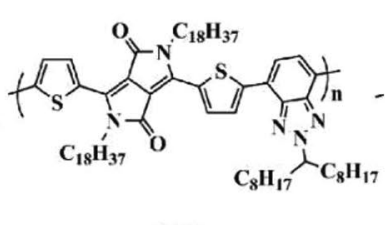

P13

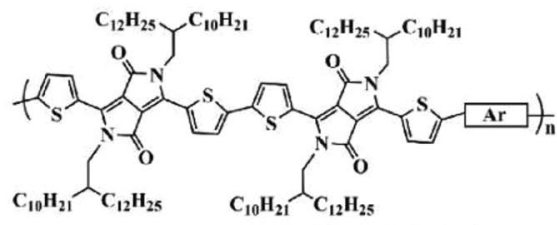

P14

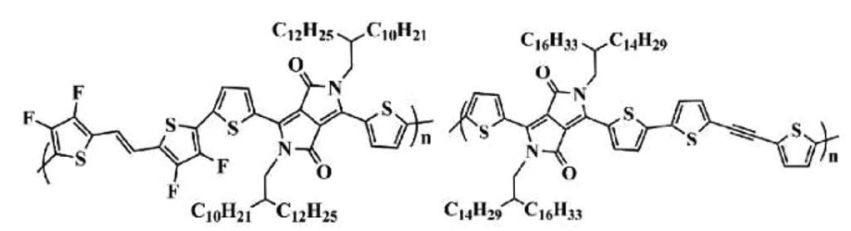

P15

P18

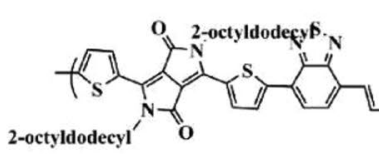<smiles></smiles>

P16

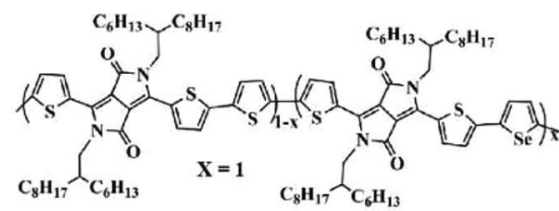

P17

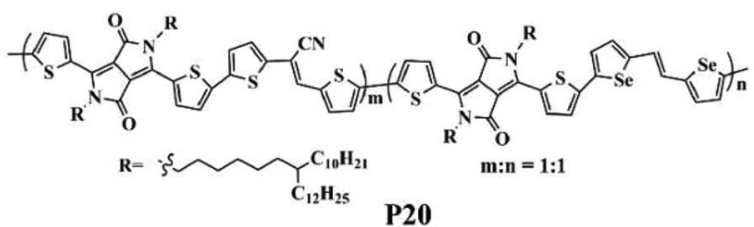

P19

$$
{ }_{\mathrm{C}_{8} \mathrm{H}_{17}}^{\mathrm{O}} \text { }_{\mathrm{C}_{10} \mathrm{H}_{21}}
$$

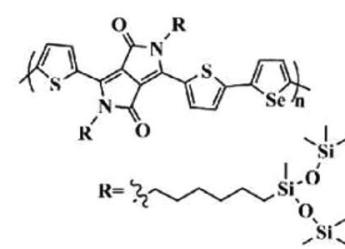

P22

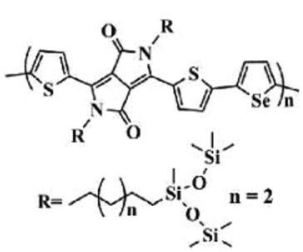

P23

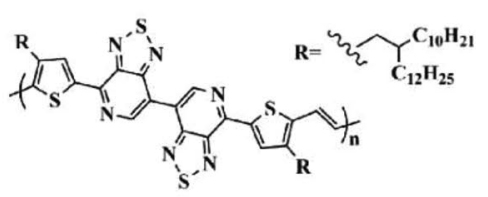

P24

Figure 15. Polymeric structures of recent selected various semiconducting polymer materials. 
selection process, particular organic polymers are capable of winding a few specific SWNTs, hence distinguishing semiconducting ones and the metallic counterparts. After that, diverse polymers such as polyfluorene derivatives (PF8, ${ }^{[253]}$ PFO-BT, ${ }^{[239]}$ PFO-BPy, ${ }^{[254]}$ etc.), poly(3-dodecylthiophene-2,5-diyl) (named P3DDT), ${ }^{[255]}$ etc., have been exploited and the nature of selective dispersion was broadly examined via molecular dynamic simulations and spectroscopic analysis. ${ }^{[256,257]}$ In 2011, Lee et al. utilized the most widely used organic materials (regioregular poly(3-alkylthiophene)s (rr-P3ATs)) to realize highly efficient sorting of SWNTs. ${ }^{[258]}$ Their method allowed direct film fabrication after a facile centrifugation process and the manufactured transistors based on sorted SWNT network displayed superior electrical performance with charge mobility of $12 \mathrm{~cm}^{2} \mathrm{~V}^{-1} \mathrm{~s}^{-1}$ and large on/off ratio of more than $10^{6}$.

During polymer sorting, it is noteworthy that removal of the residual polymers is necessary and favorable to improve the electrical performance of ambipolar transistors on the basis of SWNTs. ${ }^{[253,259]}$ Bisri et al. proposed a facile and effective approach to prepare highly purified SWNT dispersions and meanwhile remove redundant polymers efficiently. ${ }^{[253]}$ Fabricated ambipolar FETs based on aforementioned dispersions exhibited extremely large electron mobility of $3 \mathrm{~cm}^{2} \mathrm{~V}^{-1} \mathrm{~s}^{-1}$ and high on/off ratio of $10^{6}$, which indicated the prepared SWNT layers possessed no metallic counterparts. Their high purity SWNT ink and cost-effective approach to prepare it would speed up the manufacture of high-performance ambipolar transistors based on SWNTs. In addition, researchers have also discovered that both the backbones and the side chains of conjugated polymers have influences on the selection and sorting of SWNTs with various sizes and chirality, which offers more possibilities to sort and purify SWNTs in large scale. ${ }^{[260-263]}$ Gomulya et al. utilized polyfluorene derivatives with identical backbone and augmented alkyl chain lengths to sort and select SWNTs with diverse diameters (Figure 16a) and the fabricated transistors based on high-concentration SWNT network presented tremendous hole $\left(14 \mathrm{~cm}^{2} \mathrm{~V}^{-1} \mathrm{~s}^{-1}\right)$ and electron $\left(16 \mathrm{~cm}^{2} \mathrm{~V}^{-1} \mathrm{~s}^{-1}\right)$ mobilities as well as large on/off ratio of $10^{5} .^{263]}$ This effective approach displays the possibility for largescale selective differentiation.

The alignment of random nanotube networks within semiconducting layer has been confirmed to enable better electrical performance. Specifically, the mobility of transistors can be significantly improved by aligning SWNTs owing to decreased tube-to-tube resistance for short-channel transistors and optimized tube-to-tube junctions for long-channel devices. ${ }^{[227]}$ To achieve large-scale fabrication of transistors based on carbon nanotubes, simple, processable and scalable alignment strategies which are able to control the density and homogeneity of nanotubes are required. ${ }^{264,265]}$ Thus, diverse alignment methods have been developed including external-force driven approaches such as DEP, [266] liquid flow ${ }^{[267]}$ and self-assembly strategies for instance Langmuir-Schaefer deposition ${ }^{[268]}$ and Langmuir-Blodgett assembly. ${ }^{[269]}$ In addition, it is worth mentioning that the functionalized polymers can be utilized to anchor the SWNTs on electrodes during the deposition, which makes SWNTs tend to self-align. ${ }^{[269-272]}$ Recently, Derenskyi et al. exploited blade-coating technique to align nanotube networks in semiconducting channel and the manufactured transistors exhibited unprecedentedly large on/off ratio (about $10^{8}$ ) (Figure 16b). ${ }^{[255]}$

However, inherent bipolar SWNTs usually present p-type conducting behavior under air atmosphere due to the existence of oxygen, water as well as hydroxyl units in dielectric interface (capture electrons) and the usage of metal electrodes with large work function (large electron injection barrier). Therefore, scientists have proposed several techniques to realize ambipolar charge transport, for instance, utilizing high- $k$ dielectric layer, ${ }^{[273]}$ metal electrodes with small work function, ${ }^{[274,275]}$ chemical doping (electron-donating agents) ${ }^{[276,277]}$ and ionic liquid/gel gating ${ }^{[278,279]}$ can be adopted. Another valid method is to exploit dual-gate (original gate and control gate) architecture to modulate the vertical electrical field and the charge carriers within semiconducting channel and hence achieve controllable threshold voltage and charge polarity. ${ }^{[228,280]}$ Very recently, by using dual-gate transistor structures, Yu et al. demonstrated flexible transistors with symmetric bipolar properties and realized stable dominate p- or n-type performance through adjusting the control gate bias (Figure 16c,d). ${ }^{[281]}$ These progresses of sorting, doping and self-assembly strategies are advantageous for the further implementation of large-scale printed commercial electronic products on the basis of SWNTs.

\section{Inorganic Semiconducting Materials}

2D layered materials, as advent of graphene $(\mathrm{Gr})$, have received increasing attention of both basic science and industry research due to their peculiar optical, electrical, mechanical and photoelectric performance in nature. ${ }^{[282]}$ In the modern semiconductor industry, novel semiconducting 2D layered materials, such as graphene derivatives, boron nitride $(\mathrm{BN})$, transitionmetal dichalcogenides (TMDs), and black phosphorus (BP), are extensively utilized in electronic devices. Compared with traditional bulk materials, 2D layered materials have many fantastic properties. First, as the electron effective mass is negligible along the Fermi surface, the theoretical charge mobility of 2D monolayer is very high, which attracts infinite interest from device scientists. Second, the fundamental bandgap of 2D layered materials could be magically modulated when the bulk 2D materials gradually change into the monolayer ones. This property makes 2D layered materials very popular for application of electronic and photoelectric devices (e.g., FETs, light-emitting diodes (LEDs) and photovoltaic cells) with wide spectrum response. Third, the monolayer $2 \mathrm{D}$ materials are extremely sensitive to the change of environment because all atoms are exposed on the surface. In other words, the electronic and photoelectric devices based on 2D layered materials can be facilely modulated by various methods (e.g., surface treatment, ${ }^{[283]}$ chemical doping, ${ }^{[284]}$ physical process, ${ }^{[285]}$ interface, and electrode contacts $\left.{ }^{[26,287]}\right)$. Based on the two properties of 2D layered materials described above (layer-dependent bandgap and sensitivity to the environment), 2D layered materials may possess different ambipolar mechanisms compared with previously mentioned organic semiconductors. Finally, van der Waals (vdW) heterostructures could be constructed by combining two different 2D monolayers. Owing to the spin-valley coupling of carriers and moiré pattern with a superlattice potential in 
(a)
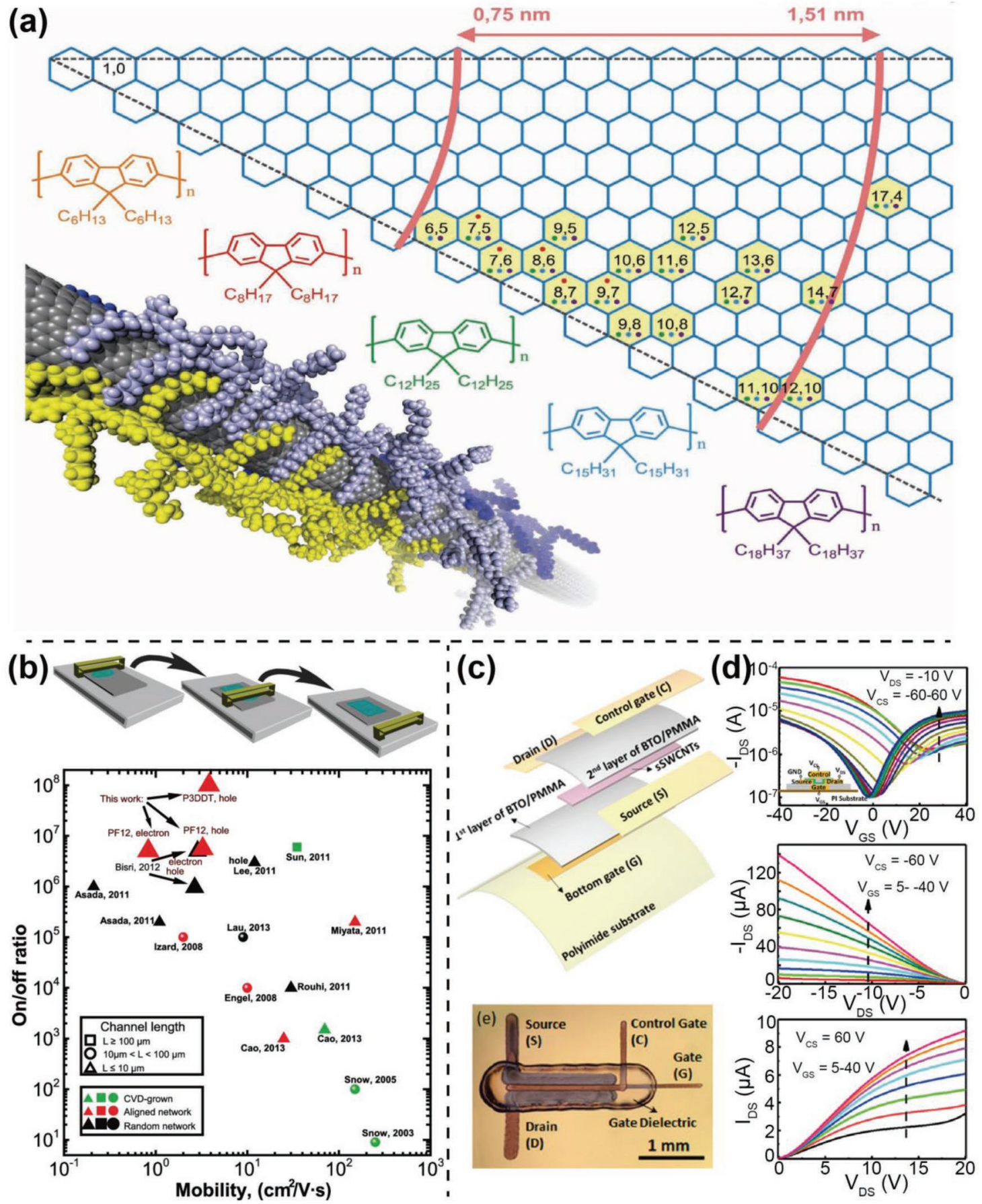

Figure 16. a) Selective solubilization of SWNTs with different diameters and chirality by exploiting various conjugated polymers with various polymer backbones or diverse alkyl chain lengths. Reproduced with permission. ${ }^{[263]}$ Copyright 2013, Wiley-VCH. b) Schematic diagram describing the procedures of blade coating; electrical performance image of transistors based on SWNTs manufactured by CVD and solution-processing methods. Reproduced with permission. ${ }^{[255]}$ Copyright 2014, Wiley-VCH. c) Schematic illustration and optical image of the printed flexible dual-gate TFT. d) Representative transfer characteristics of the printed flexible TFTs tested under various control gate biases; typical p-type (top) and n-type (bottom) output curves of the dual-gate TFTs under identical control gate bias. Reproduced with permission. ${ }^{[281]}$ Copyright 2018, American Chemical Society.

vertical vdW architectures, many new properties and special phenomena (e.g., fractal quantum Hall effect, ${ }^{[288]}$ circular polarized emission, ${ }^{[289]}$ unconventional superconductivity, ${ }^{[290]}$ and tunable Mott insulators ${ }^{[291]}$ ) can be revealed. On account of these fantastic properties of $2 \mathrm{D}$ layered materials, we then focus on the recent progress of ambipolar transistors based on 2D layered materials and vdW heterostructures.

When applied in FETs, 2D layered materials exhibit unambiguous advantages compared to traditional silicon-based materials. i) Ambipolar transistors based on ultrathin semiconducting 
layer can be achieved due to the ultrathin thickness of 2D layered materials. ii) On the basis of the flexible characteristics of monolayer or few-layer 2D semiconductors, 2D layered materials allow the development of novel flexible electronic devices. iii) Transparent devices can also be fabricated because of the ultrahigh transmittance of visible light in 2D layered materials. iv) Ambipolar transistors with low power dissipation can be realized by utilizing 2D layered materials. However, in an ambipolar transistor, the material should have both high electron mobility and hole mobility; that is, the material should possess moderate bandgap. Graphene, a zero bandgap material, is hindered due to its low on/off ratio. Therefore, plenty of researchers concentrate on ambipolar transistors on the basis of TMDs and BP.

As mentioned above, layer-dependent bandgap and sensitivity to the environment of $2 \mathrm{D}$ layered materials may make them possess various mechanisms compared with previously mentioned organic semiconductors. The band alignments are utilized to interpret ambipolar behaviors of 2D layered materials in the early stage by reason of the various work functions between metal electrodes and 2D semiconductors. Therefore, Schottky barrier is formed and then affects the injection of holes and electrons (Figure 17a). ${ }^{[292]}$ This explanation for ambipolar behaviors is similar to that for organic semiconductors. In addition, Fermi level shift which is induced by surface charge transfer doping (e.g., metal contact doping ${ }^{[293]}$ ) is also exploited to explain the ambipolar carrier conduction of 2D layered materials. The polarities of $2 \mathrm{D}$ materials are facilely modulated by employing appropriate p- and n-type dopants and thereby good ambipolar behaviors can be observed in 2D transistors under proper doping. ${ }^{[294]}$ The Fermi level shift generated by surface (a)
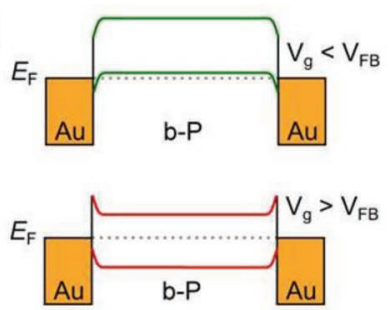

(b)

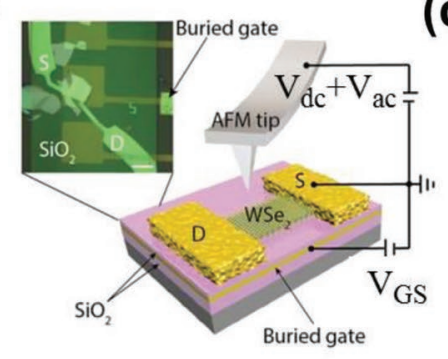

(c)

(e)

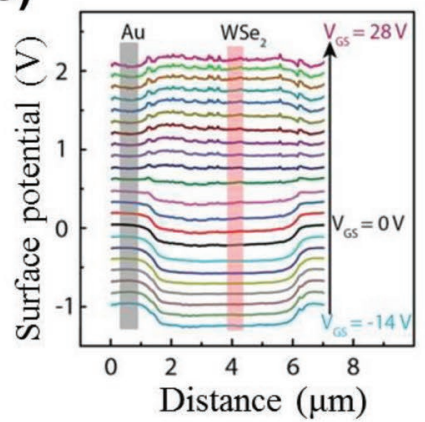

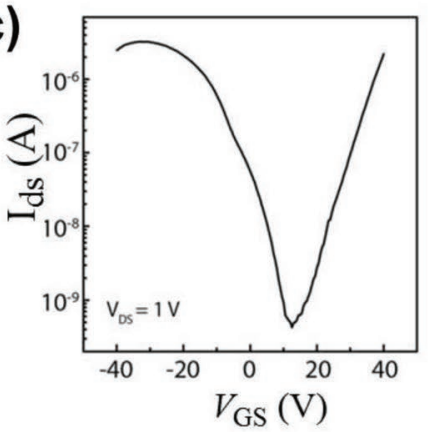
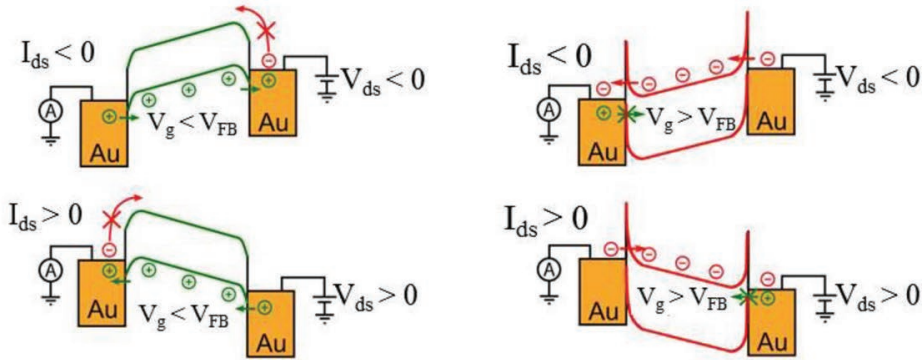

(d)

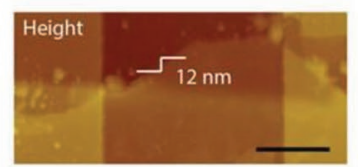

(f)

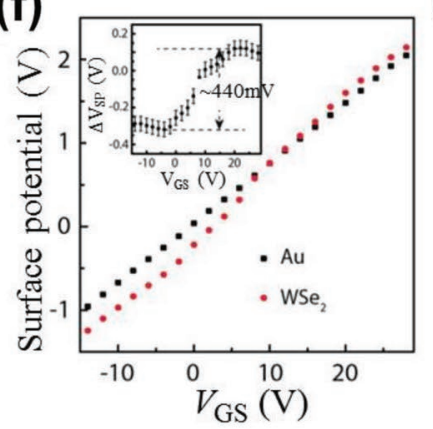

(g)

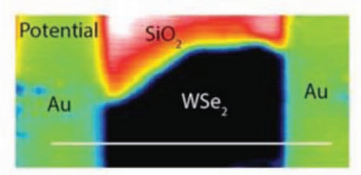

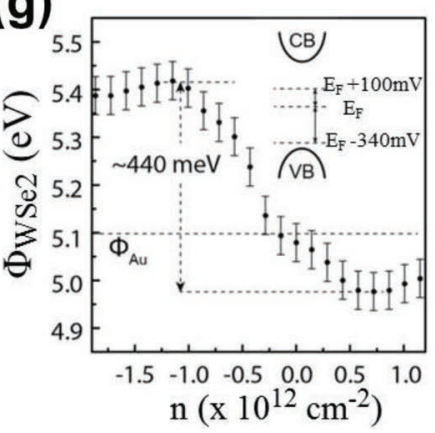

Figure 17. a) Left: Band alignment diagram and Schottky barriers at source $\left(V_{s}=0 \mathrm{~V}\right)$ and drain $\left(V_{\mathrm{g}}=0 \mathrm{~V}\right)$ contacts for gate voltages below and above the flat-band condition $\left(V_{F B}\right)$ in BP-based FET, $E_{F}$ denotes Fermi energy; middle: band diagram of the hole-only transport at $V_{\mathrm{g}}<V_{\mathrm{FB}}$ for $V_{\mathrm{Sd}}<0$ (top) and $V_{\text {sd }}>0$ (bottom); right: band diagram of the electron-only transport at $V_{\mathrm{g}}<V_{\mathrm{FB}}$ for $V_{\mathrm{sd}}<0$ (top) and $V_{\text {sd }}>0$ (bottom). Reproduced with permission. ${ }^{[292]}$ Copyright 2014, American Chemical Society. b-d) The gate-induced Fermi level shift in ambipolar WSe 2 FET. Left (b): The schematic diagram for measuring the surface potential of $\mathrm{WSe}_{2}$ and $A u$ at $V_{\mathrm{sd}}=-14-28 \mathrm{~V}$ by a Kelvin probe force microscopy; middle (c): transfer characteristics of the ambipolar WSe $\mathrm{WET}_{2}$; right (d): the AFM morphology (top) and corresponding surface potential map (bottom). e-f) The summary of surface potential on the Au electrode and $\mathrm{WSe}_{2}$ region (e), indicating the Fermi level shift as a function of gate voltage (f). g) The work function variation of WSe $\mathrm{e}_{2}$ as $V_{s d}$ changes in the transistor. The inset shows the corresponding shift of Fermi level, indicating $\mathrm{WSe}_{2}$ being modulated to be $\mathrm{n}$ - or $\mathrm{p}$-type at small gate voltage. Reproduced with permission. ${ }^{[296]}$ Copyright 2018, Springer Nature. 
charge transfer doping supports a novel ambipolar mechanism to understand ambipolar behaviors of 2D layered materials. However, it is unfeasible to interpret the layer-dependent ambipolar behaviors of 2D materials. Fortunately, Zhou et al. proposed that Fermi level shift also happened in the band alignment process among $2 \mathrm{D} \mathrm{WSe}_{2}$ with various thicknesses. ${ }^{[295]}$ Wang et al. directly investigated the dependence of Fermi level shift of 2D $\mathrm{WSe}_{2}$ on different gate voltages under Kelvin probe force microscopy, as demonstrated in Figure 17b-g. ${ }^{[296]}$ They built a model of gate-induced Fermi level shift to interpret the ambipolar principles of $2 \mathrm{D} \mathrm{WSe}_{2}$. This model also revealed that the Fermi level of some 2D layered materials could be tunable by applying a small gate voltage. Thus, these 2D materials could be modulated to display n-type, p-type, and ambipolar charge transport. Apparently, there are some unipolar 2D layered materials which are unable to be explained by this model on account of their intense Fermi level pinning effect in energy band. Therefore, a more general mechanism is eagerly required to interpret ambipolar behaviors of 2D layered materials.

\subsection{Molybdenum Dichalcogenides $\left(\mathrm{MoS}_{2}, \mathrm{MoTe}_{2}\right.$, and $\left.\mathrm{MoSe}_{2}\right)$}

As a novel class of 2D layered materials, TMDs possess the common formula $\mathrm{MX}_{2}$, where $\mathrm{M}$ is a kind of transition metals from group IV-VIII (e.g., Mo, Nb, W, Re, and Pt), and X represents a kind of chalcogen elements (e.g., S, Te, and Se). 2H-type molybdenum disulfide $\left(2 \mathrm{H}-\mathrm{MoS}_{2}\right)$ is a typical and stable semiconducting TMD layered compound. Its excellent optical, electrical and photoelectric characteristics have received great attention from researchers for the application of $\mathrm{MoS}_{2}$ in high performance nanoelectronics. First, the fundamental bandgap of $2 \mathrm{H}-\mathrm{MoS}_{2}$ gradually changes from an indirect gap $(\approx 1.2 \mathrm{eV})$ for the bulk to a direct gap for the monolayer $(\approx 1.9 \mathrm{eV}) .{ }^{[297]}$ This layer-dependent optical property makes it possess tunable optical absorption as well as good electrical performance. When applied in transistors, the mobility of $2 \mathrm{H}-\mathrm{MoS}_{2}$ could be more than 200 and $500 \mathrm{~cm}^{2} \mathrm{~V}^{-1} \mathrm{~s}^{-1}$ which was obtained from high- $\kappa$ dielectric $\left(\mathrm{HfO}_{2}\right.$ and $\left.\mathrm{Al}_{2} \mathrm{O}_{3}\right)$ capped monolayer and multilayer $\mathrm{MoS}_{2},{ }^{[298,299]}$ and the on/off ratio of these devices was more than $10^{6} \cdot{ }^{[300]}$ Therefore, the ambipolar transistors based on $2 \mathrm{H}-\mathrm{MoS}_{2}$ have attracted much attention.

Diverse efforts such as introducing overlayer and changing substrate have been taken to change intrinsic electrical properties of $\mathrm{MoS}_{2}$ and hence make it display ambipolar characteristics. ${ }^{[301,302]}$ Thanks to the chemical stability of $\mathrm{MoS}_{2}$, the most common method of introducing overlayer is to fabricate electric double-layer (EDL) transistors, in which ionic liquid is introduced to the top of $\mathrm{MoS}_{2}$ flakes. The ionic liquid overlayer usually functions as a top gate and can conveniently form nanometer-scale thickness of electric double layer under a low applied voltage. This electric double layer can exhibit extremely large gate capacitance and hence enable a remarkably drop of the operating gate voltage. On the other hand, electric double layers can also induce ambipolar carrier transport and accumulate extremely large density of carriers in both conduction and valence bands of TMDs. ${ }^{[303]}$ As a consequence, ambipolar transistors based on TMDs can be successfully manufactured with high on/off current ratio.
Zhang et al. demonstrated that a mechanically exfoliated $\mathrm{MoS}_{2}$ thin flake displayed ambipolar charge transport by exploiting ionic liquid gate (Figure 18a). ${ }^{[302]}$ Unlike the conventional n-type bulk $\mathrm{MoS}_{2}, \mathrm{MoS}_{2}$ thin flake exhibited high mobilities for both electrons $\left(44 \mathrm{~cm}^{2} \mathrm{~V}^{-1} \mathrm{~s}^{-1}\right)$ and holes $\left(86 \mathrm{~cm}^{2} \mathrm{~V}^{-1} \mathrm{~s}^{-1}\right)$ (Figure 18b). In addition, the on/off current ratio of the aforementioned ambipolar transistors was as high as $10^{2}$ for both hole and electron conduction. It was also found that the ingenious utilization of frozen ionic liquid could introduce a chance to form or eliminate the $\mathrm{p}-\mathrm{n}$ junction by controlling operation temperature. The movement of ions was fixed in the ionic liquid ((N,N-diethyl- $N$-methyl- $N$-(2-methoxyethyl) ammonium bis(trifluoromethylsulfonyl) imide, DEME-TFSI) when the temperature was below glass transition temperature. Then, the $\mathrm{p}-\mathrm{n}$ junction would form under the changes of electric field between source and drain electrodes (Figure 18c). ${ }^{[300]}$ Moreover, these junctions showed unambiguous rectifying characteristics when $\mathrm{p}-\mathrm{n}$ junctions formed under different initial bias conditions (Figure 18d). The fabrication of $\mathrm{p}-\mathrm{n}$ junction within monolayer $\mathrm{MoS}_{2}$ channel could be expected to stimulate a tremendous development of optoelectronic devices. The ionic liquid could effectively reduce Schottky barrier thickness at the $\mathrm{MoS}_{2} /$ metal contacts and significantly enhance ambipolar performance. Perera et al. reported ambipolar transistors with on/off current ratio of more than $10^{7}$ and $10^{4}$ for electrons and holes, respectively. ${ }^{[304]}$ The electron mobility of the aforementioned transistors exceeded $60 \mathrm{~cm}^{2} \mathrm{~V}^{-1} \mathrm{~s}^{-1}$ at $250 \mathrm{~K}$.

All these results motivate a promising application potential of $\mathrm{MoS}_{2}$ thin flake in optoelectronic devices. However, the large-area 2D thin flake is always fabricated by a chemical vapor deposition (CVD) technique, which is potential to realize commercial application of $2 \mathrm{D}$ thin flakes. Therefore, fabricating ambipolar transistors based on large-area CVDgrown thin flake is of great significance. Ponomarev et al. demonstrated that large-area $\mathrm{MoS}_{2}$ monolayers grown by CVD technique could be utilized in ambipolar EDL transistors. ${ }^{[305]}$ Meanwhile, electroluminescence was observed from the device due to the exciton recombination in CVD-grown $\mathrm{MoS}_{2}$ monolayer. These results were useful to identify the in-gap states which could serve as traps for holes and offered a controllable and scalable technique to fabricate large-area transistors.

Recently, electrolyte and ionic liquid gating have attracted widespread attention in superconducting field. ${ }^{[303,306-308]}$ Ye et al. observed superconductivity dome in $\mathrm{MoS}_{2}$ flake by exploiting electric double layer technique, which heralded the coming of gate-controlled superconductivity. ${ }^{[303]}$ Then the adjustable superconducting behaviors were discovered by $\mathrm{Li}$ et al. with a electrolyte gate. ${ }^{[308]}$ Furthermore, Costanzo et al. first demonstrated the persistent limit of superconductivity in exfoliated $\mathrm{MoS}_{2}$ flake with the frozen ionic liquid gate. ${ }^{[306]}$ Jin et al. demonstrated that both two types of charge carriers were existent in electric double layer transistor. ${ }^{[307]}$ This result indicates that it is possible to realize ambipolar superconductivity in 2D layer materials. In other words, n-type superconductivity (electron-dominant) and p-type superconductivity (holedominant) may be realized. In conclusion, the combination of advanced ionic liquid gating technique and ambipolar characteristics of 2D layered materials can promote the development 
(a)

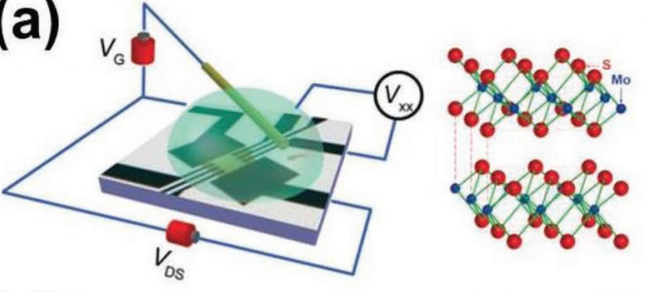

(d)

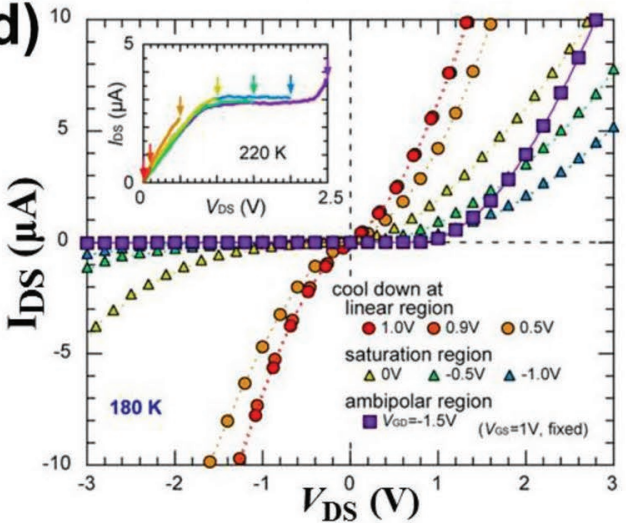

(b)

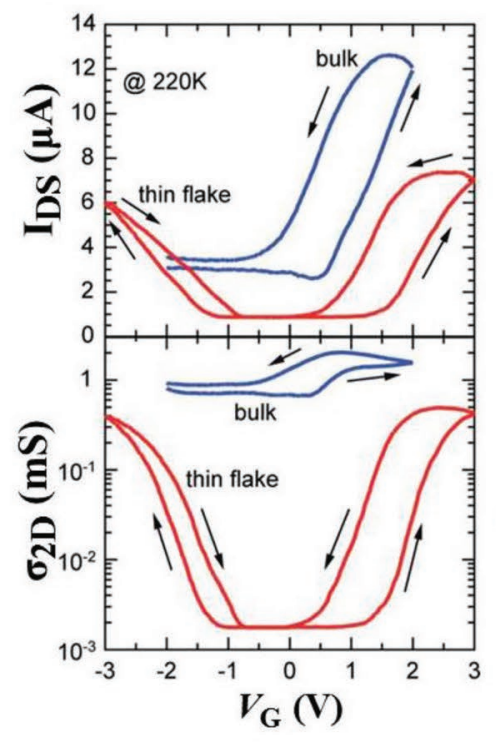

(c)
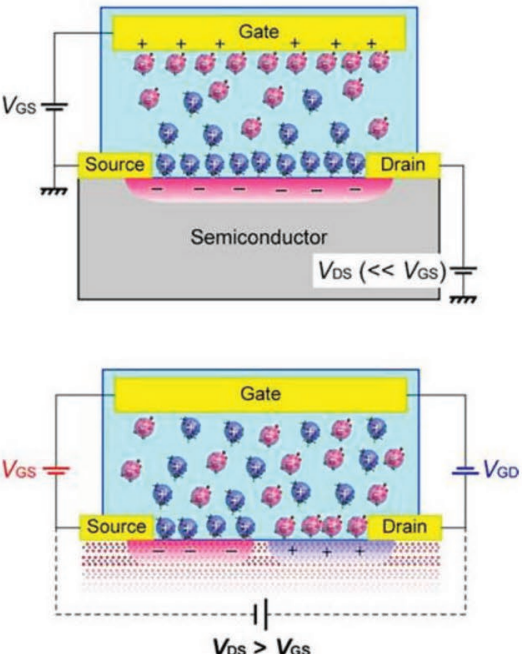

$V_{o s}>V_{G S}$ (e)

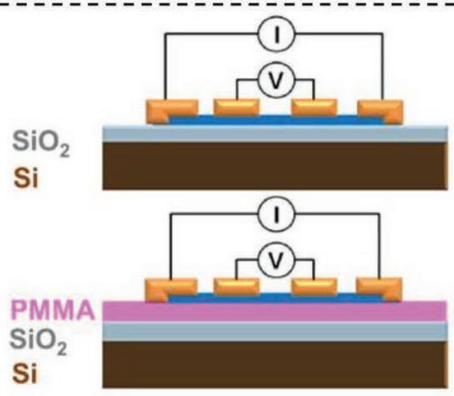

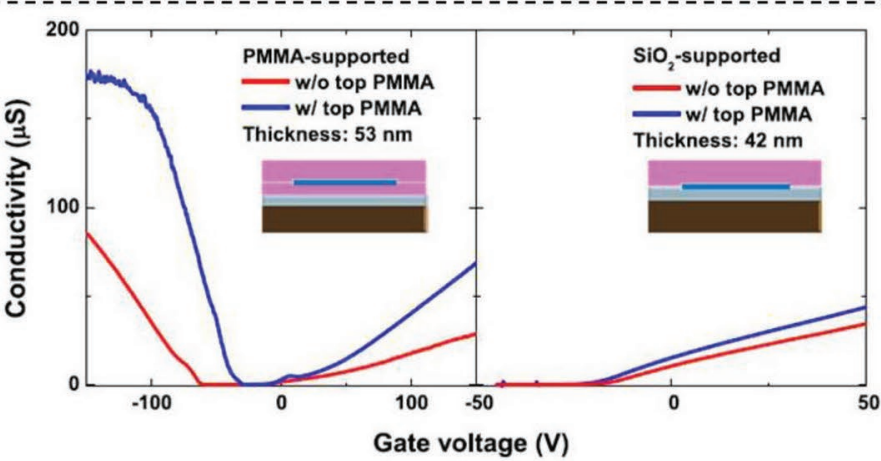

Figure 18. The electrical behavior modulation for 2D MoS 2 with EDLT and SE techniques. a) Schematics of a MoS 2 thin flake patterned with metal electrodes and a top ionic liquids gate. b) Transfer curve of bulk and thin flake MoS $_{2}$ EDLTs; top: output curve of the channel current $I_{D s}$; bottom:

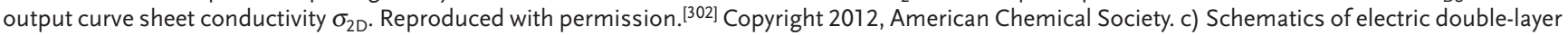
transistors (EDLTs); top: unipolar; bottom: ambipolar. d) I-V characteristics of EDLTs at $180 \mathrm{~K}$; inset: output curve of EDLTs measured at $220 \mathrm{~K}$; arrows showed the devices were cooled from 220 to $180 \mathrm{~K}$ at the current bias voltages to reduce ionic motion; the $I-V$ characteristics at $180 \mathrm{~K}$ are related to the initial bias voltages condition; symmetric $I-V$ characteristics: cooling the device when $V_{D S}$ resides in the linear region; asymmetry $I-V$ characteristics: cooling the device when $V_{D S}$ resides in the saturation region; rectifying I-V characteristics: cooling the device when $V_{D S}$ resides in the ambipolar region. Reproduced with permission. ${ }^{[300]}$ Copyright 2013, American Chemical Society. e) Schematics of four-probe MoS 2 devices with (bottom) and without (top) PMMA layer; typical conductivity $\left(V_{\mathrm{g}}\right)$ characteristics for devices without (red curve) and with (blue curve) PMMA top coating for a devices with and without PMMA-supported layer. Reproduced with permission. ${ }^{[301]}$ Copyright 2013, American Institute of Physics.

of ambipolar superconducting field and hence bring a series of novel applications in electronics.

Changing substrate of thin-film transistors is another method to affect its electrical performance. The introduction of disorder between TMDs and the substrate, such as shortranged disorder and long-ranged charge disorder due to the roughness and chemical bond, would decrease carrier mobility. On the contrary, carrier mobility could be augmented by dielectric screening effects. ${ }^{[309]}$ For example, a PMMA layer could be employed to obtain ambipolar characteristics of $\mathrm{MoS}_{2}$. Bao et al. demonstrated that multilayer $\mathrm{MoS}_{2}$ on PMMA could display ambipolar carrier conduction while that on $\mathrm{SiO}_{2}$ showed unipolar performance (Figure 18e). ${ }^{[301]}$ The mobility could be up to $470 \mathrm{~cm}^{2} \mathrm{~V}^{-1} \mathrm{~s}^{-1}$ (electrons) and $480 \mathrm{~cm}^{2} \mathrm{~V}^{-1} \mathrm{~s}^{-1}$ (holes).
Furthermore, chemical p-type doping was also introduced into $\mathrm{MoS}_{2}$ flakes to realize p-type or ambipolar characteristics. Choi et al. introduced hole-injecting $\mathrm{MoO}_{x} \mathrm{NPs}$ (oxygen doping) to the surface of $\mathrm{MoS}_{2}$ flakes, which could finally lead to ambipolar behaviors. ${ }^{[310]}$

2H-type molybdenum ditelluride $\left(\mathrm{MoTe}_{2}\right)$ is also a common 2D material. The indirect bandgap of bulk $\mathrm{MoTe}_{2}$ is $\approx 1.0 \mathrm{eV} .^{[311]}$ Theoretically, its mobility can be as high as $200 \mathrm{~cm}^{2} \mathrm{~V}^{-1} \mathrm{~s}^{-1}$ at room temperature. ${ }^{[312]}$ The direct bandgap of $\mathrm{MoTe}_{2}$ monolayer is $1.07 \mathrm{eV}$ due to the induced quantum confinement. ${ }^{[313]}$ Unlike $\mathrm{MoS}_{2}$, mechanically exfoliated $\mathrm{MoTe}_{2}$ was demonstrated to possess intrinsic and thickness-dependent ambipolar characteristics. ${ }^{[312,314,315]}$ In these devices, on/off current ratio could reach $5 \times$ $10^{2}$ (n-type regime) and $2 \times 10^{3}$ (p-type regime). However, slightly 
low mobilities of $0.03 \mathrm{~cm}^{2} \mathrm{~V}^{-1} \mathrm{~s}^{-1}$ (electrons) and $0.30 \mathrm{~cm}^{2} \mathrm{~V}^{-1} \mathrm{~s}^{-1}$ (holes) were obtained. Moreover, a dual-top-gated device with two equivalent top gates was developed by Lin et al. The polarity of the bias voltage could be controlled by just adjusting one of the top gates (Figure 19a). ${ }^{[314]}$ When the $V_{\text {tgs }}$ was negative, the transistor displayed p-type characteristic due to the injected and accumulated holes within the semiconducting channel. Otherwise, the transistor presented n-type characteristic. By changing the mechanically exfoliated $\mathrm{MoTe}_{2}$ channel thickness, Fathipour et al. demonstrated a higher on/off current ratio $\left(10^{5}\right.$ with 6 monolayers) and a higher mobility $\left(6 \mathrm{~cm}^{2} \mathrm{~V}^{-1} \mathrm{~s}^{-1}\right) \cdot{ }^{[315]}$
Other efforts were devoted to exploiting chemical p-type doping and surface charge transfer doping (SCTD) to realize ambipolar characteristics of $\mathrm{MoTe}_{2}$. As mentioned above, introducing oxygen atoms into crystal lattice of TMDs could form p-type $\mathrm{MoO}_{x}$ phase. The $\mathrm{MoO}_{x}$ phase could inject holes and gave rise to p-type or ambipolar characteristics of $\mathrm{MoS}_{2}$. This method is also suitable for $\mathrm{MoTe}_{2}$ to achieve p-type or ambipolar behaviors. Qu et al. was successful to modulate $\mathrm{MoTe}_{2}$ transistors to show unipolar p-type, n-type, or ambipolar performance by introducing $\mathrm{BV}$-doping (BV: benzyl viologen) to $\mathrm{MoTe}_{2}$ (Figure 19b). ${ }^{[284]}$ Marvelously, a proper $\mathrm{Al}_{2} \mathrm{O}_{3}$ capping
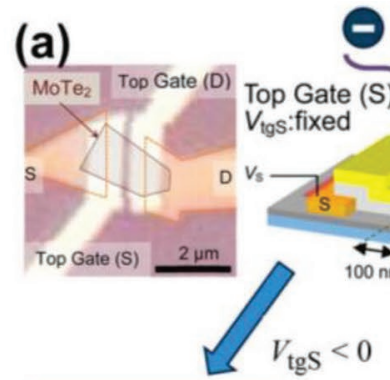

or 4
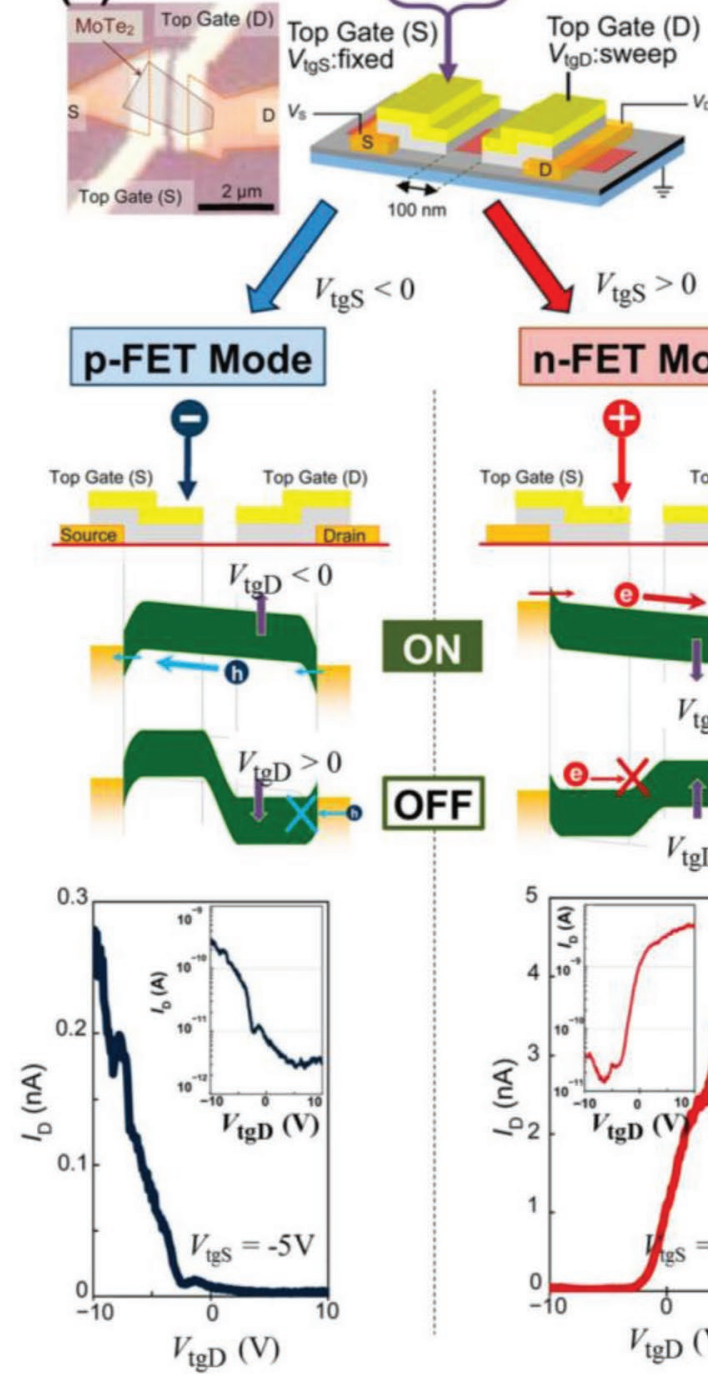
o:sweep
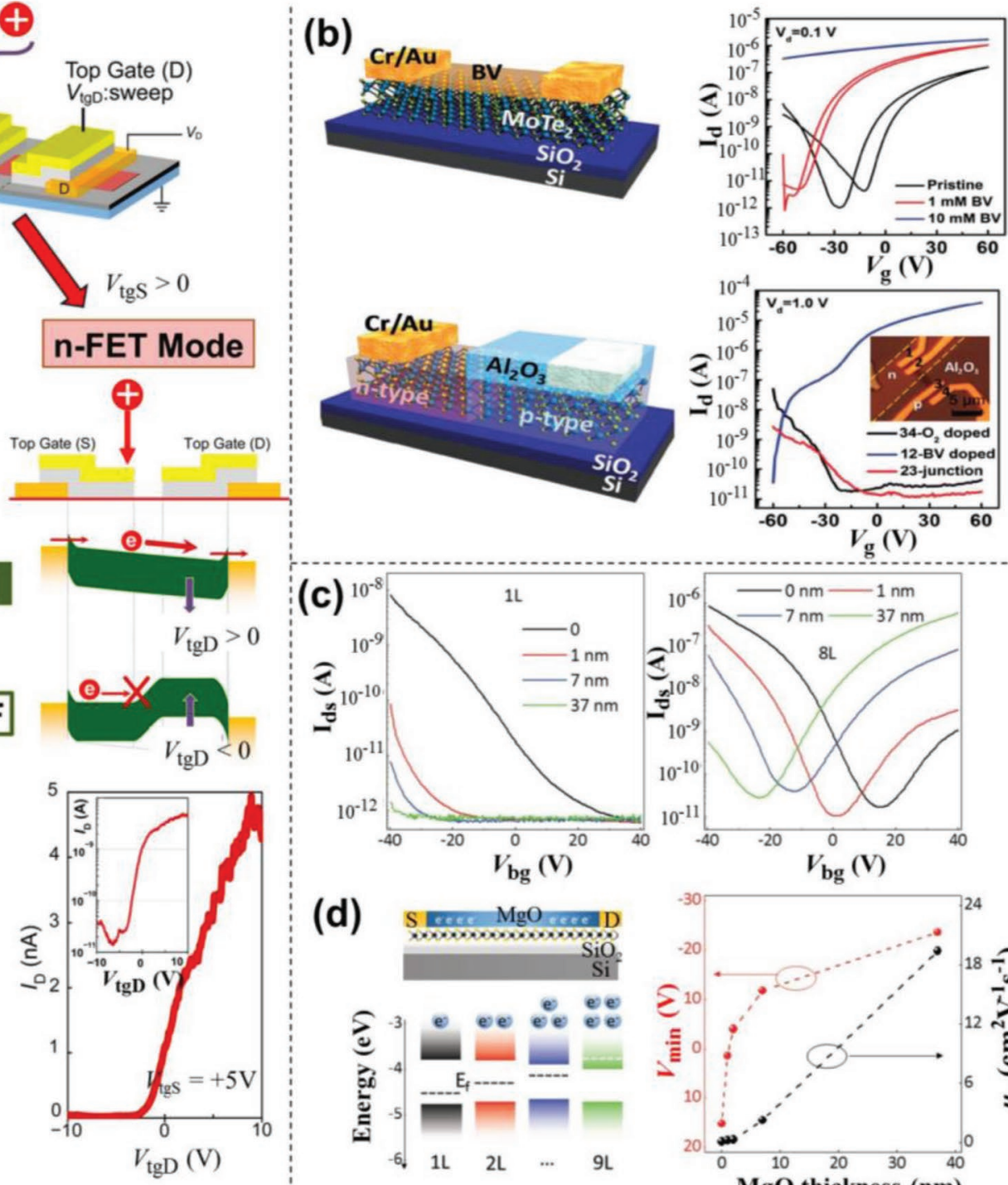

(d)

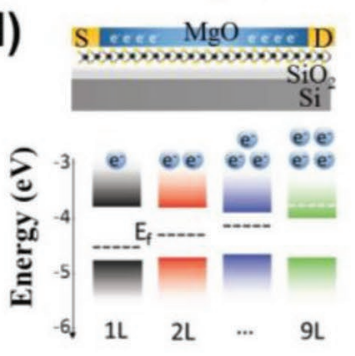

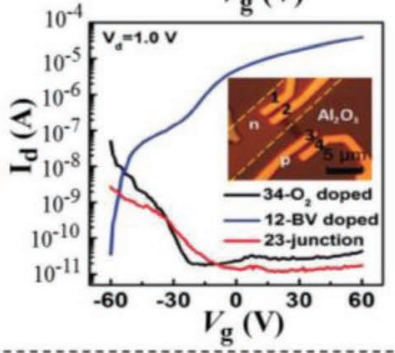
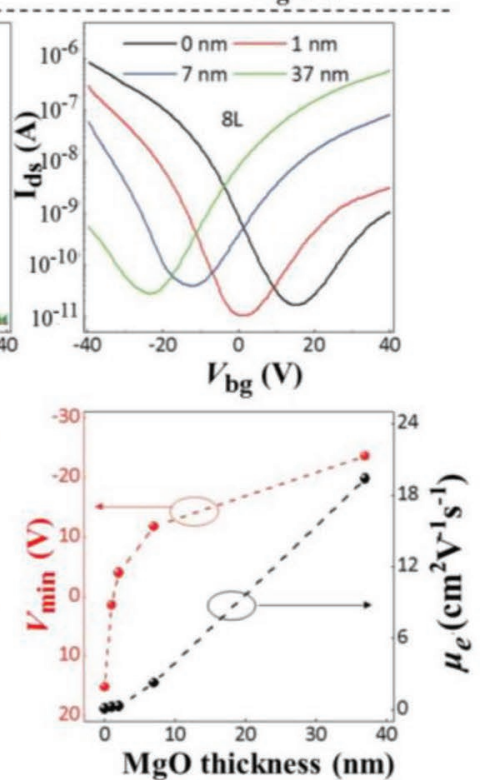

Figure 19. The electrical behavior modulation for 2D MoTe 2 with dual-top-gate, CD, and SCTD techniques. a) Optical micrograph, schematic structure, $\mathrm{p}$ - and $\mathrm{n}$-FET modes of a dual-top-gate transistor; $V_{\text {tgs }}<0$, the $\mathrm{p}$-FET mode is matched, and agreed with experimental results; otherwise the $\mathrm{n}$-FET mode is matched, and agreed with corresponding experimental results. Reproduced with permission. ${ }^{[314]}$ Copyright 2015, American Chemical Society. b) Schematic diagram of BV doping process devices (top) and lateral $\mathrm{p}-\mathrm{n}$ junction devices with an $\mathrm{Al}_{2} \mathrm{O}_{3}$ mask (bottom); transfer curves with different BV dopant concentration (top); comparison of transfer curves for devices with $\mathrm{O}_{2}$ doped, $\mathrm{BV}$ doped and p-n junction; inset: optical microscopy image of the device. Reproduced with permission. ${ }^{[284]}$ Copyright 2017, Wiley- $\mathrm{VCH}$. c) Transfer characteristics of the monolayer and the 8L MoTe 2 FET with increasing thickness of $\mathrm{MgO}$ cover layers. $\mathrm{d}$ ) The schematic structure of the MgO doped $\mathrm{MoTe}_{2} \mathrm{FET}$; the schematic of band structures of different thickness $\mathrm{MoTe}_{2}$ flakes; the calculated movement of the $V_{\min }$ and the electron mobility of the $8 \mathrm{~L} \mathrm{MoTe} 2$ FET related to the MgO film thickness. Reproduced with permission. ${ }^{[316]}$ Copyright 2018, Wiley-VCH. 
could further improve the n-type electric behavior as well as form a $\mathrm{p}-\mathrm{n}$ junction in the transistor. As a consequence, the hole and electron mobilities could be increased to 41 and $80 \mathrm{~cm}^{2} \mathrm{~V}^{-1} \mathrm{~s}^{-1}$ and the on/off current ratio also reached $10^{5}$. Unlike chemical p-type doping, SCTD is a nondestructive and efficient technique to achieve reliable doping in 2D semiconductors and has been widely employed in surface functionalization engineering. MgO film was an efficient SCTD for 2D $\mathrm{MoTe}_{2}$. In addition, by carefully adjusting the thickness of $\mathrm{MgO}$ film and the number of $\mathrm{MoTe}_{2}$ layers, the carrier polarity of $\mathrm{MoTe}_{2}$ transistors could be reversely controlled from $\mathrm{p}$ - to n-type and the electron mobility was enhanced, as shown in Figure 19c,d. Based on thickness-dependent SCTD method, Luo et al. successfully modulated carrier mobilities and ambipolar characteristics of $\mathrm{MoTe}_{2}$ transistors. ${ }^{[316]}$

Molybdenum diselenide $\left(\mathrm{MoSe}_{2}\right)$ is an air stable TMD layered material. The bulk $\mathrm{MoSe}_{2}$ has an indirect bandgap $(1.1 \mathrm{eV})$ while a monolayer $\mathrm{MoSe}_{2}$ has a wider direct bandgap of $1.55 \mathrm{eV}^{[313]}$ The transistors based on $\mathrm{MoSe}_{2}$ display fantastic thickness-dependent characteristics. Li et al. reported that the monolayer $\mathrm{MoSe}_{2}$ prepared by an atmospheric pressure CVD exhibited n-type characteristic with a high on/off current ratio $\left(\approx 10^{6}\right)$ and a high electron mobility $\left(\approx 19 \mathrm{~cm}^{2} \mathrm{~V}^{-1} \mathrm{~s}^{-1}\right) \cdot{ }^{[317]}$ Interestingly, bilayer $\mathrm{MoSe}_{2}$ showed ambipolar behaviors with an on/off current ratio of $\approx 10^{5}$, a hole mobility of $\approx 9 \mathrm{~cm}^{2} \mathrm{~V}^{-1} \mathrm{~s}^{-1}$ and an electron mobility of $\approx 65 \mathrm{~cm}^{2} \mathrm{~V}^{-1} \mathrm{~s}^{-1}$. When the thickness of $\mathrm{MoSe}_{2}$ continuously increased to about 7 layers, the transistor showed ambipolar behaviors. ${ }^{[318]}$ Furthermore, monolayer $\mathrm{MoSe}_{2}$ could realize ambipolar charge transport by employing EDL technique. ${ }^{[319,320]}$

\subsection{Tungsten Dichalcogenides $\left(\mathrm{WS}_{2}, \mathrm{WSe}{ }_{2}\right.$, and $\left.\mathrm{WTe}_{2}\right)$}

Tungsten disulfide ( $\mathrm{WS}_{2}$ ) is also a commonly used TMD layered material and possesses indirect bandgap of 1.0, 2.14, and $1.82 \mathrm{eV}$ for bulk, monolayer and bilayer structure, respectively. ${ }^{[321]}$ In order to achieve ambipolar behavior in $\mathrm{WS}_{2}$ semiconductor, researchers have made tremendous efforts. Hwang et al. demonstrated that $\mathrm{WS}_{2}$ thin flake prepared by CVD technique presented ambipolar behavior and a high on/off current ratio $\left(\approx 10^{5}\right)$ in Schottky-barrier transistors. ${ }^{[322]}$ The mechanically exfoliated $\mathrm{WS}_{2}$ thin flake based on EDL technique also displayed ambipolar carrier conduction. ${ }^{[323]}$ The mobilities were $\approx 20 \mathrm{~cm}^{2} \mathrm{~V}^{-1} \mathrm{~s}^{-1}$ (electrons) and $\approx 90 \mathrm{~cm}^{2} \mathrm{~V}^{-1} \mathrm{~s}^{-1}$ (holes). Another remarkable research about $\mathrm{WS}_{2}$-based transistors is how to get balanced charge mobilities of both two types of charge carriers. Fortunately, the thickness of $\mathrm{WS}_{2}$ thin flake was found to influence the asymmetric transport characteristics of the ambipolar transistors. ${ }^{[321]}$ In addition, the thickness of $\mathrm{WS}_{2}$ thin flake also influences the mobility values. Compared with monolayer $\mathrm{WS}_{2}$ $\left(\approx 44\right.$ and $43 \mathrm{~cm}^{2} \mathrm{~V}^{-1} \mathrm{~s}^{-1}$ for electrons and holes, respectively), the bilayer $\mathrm{WS}_{2}$ showed reduced electron mobility $\left(\approx 19 \mathrm{~cm}^{2} \mathrm{~V}^{-1} \mathrm{~s}^{-1}\right)$ and hole mobility $\left(\approx 12 \mathrm{~cm}^{2} \mathrm{~V}^{-1} \mathrm{~s}^{-1}\right)$. The similar phenomenon was also found in the device based on $\mathrm{MoSe}_{2} \cdot{ }^{[317]}$

Tungsten diselenide ( $\mathrm{WSe}_{2}$ ) is also an important member of TMD layered semiconductors. Particularly, the mechanically exfoliated $\mathrm{WSe}_{2}$ is naturally ambipolar with a high hole mobility $\left(\approx 250 \mathrm{~cm}^{2} \mathrm{~V}^{-1} \mathrm{~s}^{-1}\right)$, a low electron mobility and a high on/off current ratio $\left(>10^{6}\right)$ when applied in transistors with different contact electrodes. ${ }^{[286,287]}$ Its bandgap is thickness-dependent and can be extracted from ambipolar transistors on the basis of EDL technique. ${ }^{[324]}$ The bandgaps are 1.02 and $1.63 \mathrm{eV}$ for bulk and monolayer $\mathrm{WSe}_{2}$, respectively. In addition, the ambipolar characteristic can also be controlled by operation temperature, ferroelectric top gate dielectric (poly(vinylidenefluoride-cotrifluoroethylene) (P(VDF-TrFE))) as well as channel thickness of $\mathrm{WSe}_{2} \cdot{ }^{[296,325,326]}$ Recently, Pudasaini et al. achieved controllable charge type in FETs on the basis of $\mathrm{WSe}_{2}$ semiconducting channel and $\mathrm{Cr} / \mathrm{Au}$ contacts via tuning the thickness of semiconducting channel and exploiting remote oxygen plasma surface treatment. ${ }^{[283]}$ By changing the thickness of semiconducting channel, the transport performance of $\mathrm{WSe}_{2}$ transistors could evolve from n-type (more than $5 \mathrm{~nm}$ ) to ambipolar (about $4 \mathrm{~nm}$ ) or p-type (less than $3 \mathrm{~nm}$ ), which originated from the changeable energy gaps of $\mathrm{WSe}_{2}$ as well as carrier band offsets. Besides, by utilizing remote oxygen plasma treatment to induce hole doping, the effective hole mobility could be augmented by three orders of magnitude. Their methods could also be employed in other TMDs for efficient carrier type control. The $\mathrm{WSe}_{2}$ thin film prepared by MBE with layer-by-layer epitaxial growth also showed ambipolar behaviors. ${ }^{[327]}$ A dual gate was employed to modulate the carrier density in two sides of the $\mathrm{WSe}_{2}$ monolayer. As shown in Figure $20 \mathrm{a}-\mathrm{d}$, by adjusting the dual-gate voltage, ambipolar performance could be achieved in various conducting regimes ( $\mathrm{NN}, \mathrm{PP}, \mathrm{PN}$, and $\mathrm{NP}$ configurations) due to the electrostatic doping. ${ }^{[13]}$

Finally, tungsten ditelluride (WTe ${ }_{2}$ ), an interesting TMD layered semiconductor, displayed an ambipolar gate-dependent conductance due to the perfect compensation of electron and hole bands when it was applied in positive magnetoresistance (MR) transistors. ${ }^{[328]}$

In addition to common TMDs (molybdenum dichalcogenides and tungsten dichalcogenides), some other TMD materials are also found with ambipolar behaviors. For example, $\mathrm{PtSe}_{2}$, a special kind of TMD materials, has strong layer-dependent semiconductor-to-semimetal evolution. When applied in FETs, PtSe displayed an ultrahigh electron mobility of $210 \mathrm{~cm}^{2} \mathrm{~V}^{-1} \mathrm{~s}^{-1}$ at room temperature. ${ }^{[329]}$ After the thickness of $\mathrm{PtSe}_{2}$ being optimized, transistors exhibited relatively balanced ambipolar carrier conduction. More success of TMD-based ambipolar transistors with higher charge mobilities is expected.

\subsection{Black Phosphorus}

Black phosphorus, a novel 2D semiconductor, has attracted more and more attention for photoelectric and electronic devices (e.g., photodetectors, transistors, and resistive random access memories (RRAMs)) due to its easily solvent-exfoliation-electronic-grade, exotic optical and electronic properties. ${ }^{[292,330-332]}$ The fundamental bandgap of BP gradually changes from $0.3 \mathrm{eV}$ in the bulk to $2.0 \mathrm{eV}$ in the monolayer.

In order to achieve and tune ambipolar behaviors in BP-based transistors, several approaches such as modulation of work function of metal electrodes, ${ }^{[333-335]}$ dielectric overlayer and SCTD ${ }^{[336-338]}$ are exploited. Based on the work function of metal contact, Das et al. and Du et al. found out that 
(a)

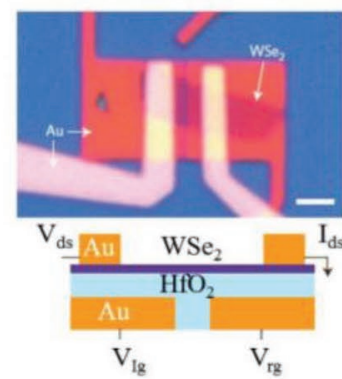

(e)

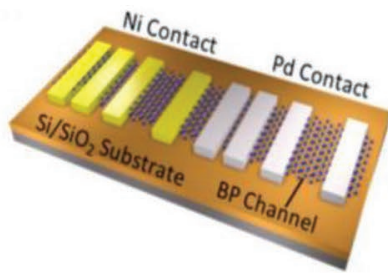

(b)

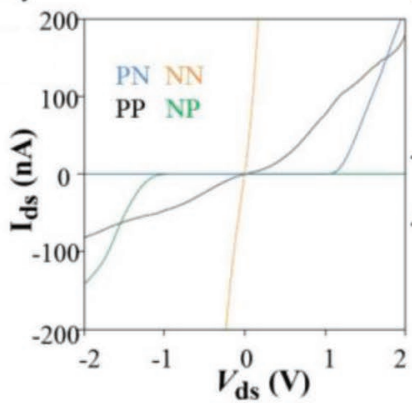

(f)

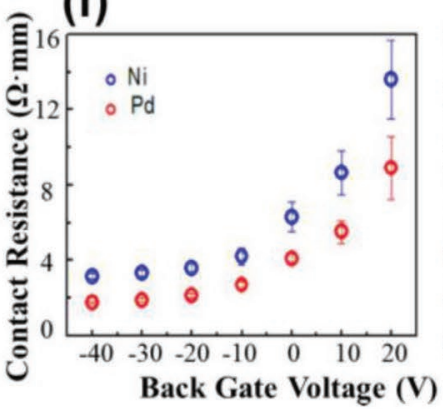

(c)

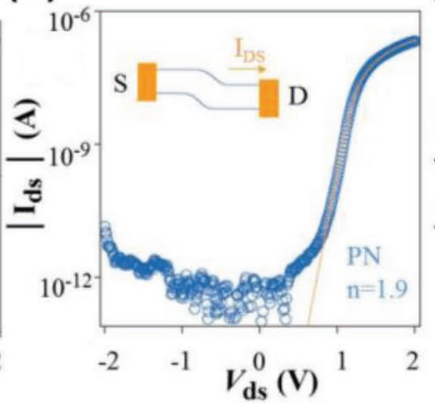

(d)

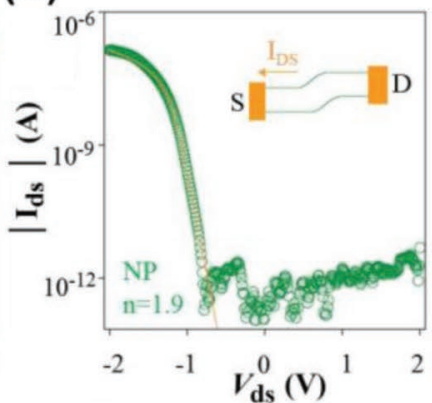

(g)

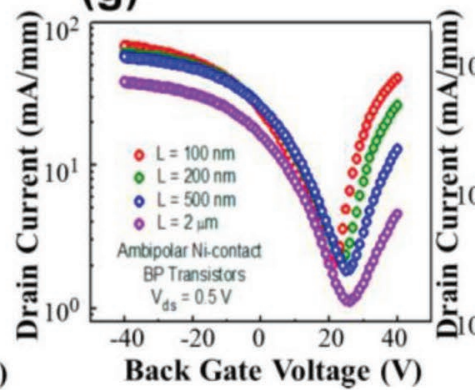

(h)

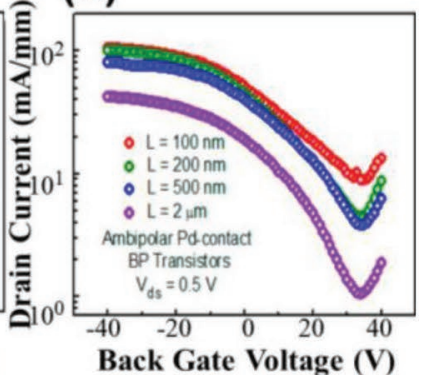

Figure 20. The electrical behavior modulation for $2 \mathrm{D} \mathrm{WSe_{2 }}$ with dual-bottom-gate (ED technique) and 2D BP with different contact metals. a) Optical micrograph (top) and schematic structure (bottom) of monolayer $\mathrm{WSe}_{2}$ devices with dual bottom gate. b) Transport characteristics of four doping configurations of the device: NN, PP, PN, and NP; NN: both gates were set to $10 \mathrm{~V}$; PP: both gates were set to $-10 \mathrm{~V} ; \mathrm{PN}$ : $V_{\mathrm{lg}}$ was set to $\pm 10 \mathrm{~V}$; NP: $V_{\mathrm{rg}}$ was set to \pm 10 V. c) Semilogarithmic plots and schematic band diagrams of the device for PN configurations. d) Semilogarithmic plots and schematic band diagrams of the device for NP configurations. Reproduced with permission. [13] Copyright 2014, Nature Publishing Group. e) Schematic structure of device configurations with various channel lengths for both $\mathrm{Ni} / \mathrm{Au}$ and $\mathrm{Pd} / \mathrm{Au}$ contacts. $\mathrm{f}$ ) Contact resistance for both $\mathrm{Ni}$ and $\mathrm{Pd}$ contact metals at various gate biases. g) $I-V$ transfer characteristic of Ni contact BP FETs with various channel lengths. h) $I-V$ transfer characteristic of Pd contact BP FETs with various channel lengths. It should be noted that transistors with a channel length of $3 \mu \mathrm{m}$ show p-type for both $\mathrm{Ni}$ and Pd contacts. Reproduced with permission. ${ }^{[334]}$ Copyright 2014, American Chemical Society.

the Schottky barrier heights of metal/BP interface led to contact resistance of the devices. BP transistors based on titanium (Ti), palladium $(\mathrm{Pd})$ and nickel $(\mathrm{Ni})$ electrodes showed ambipolar characteristics (Figure 20e-h). ${ }^{[33,334]}$ The on/off current ratio could reach $10^{4}$ for hole conduction and $10^{3}$ for electron conduction. The mobilities were $\approx 38 \mathrm{~cm}^{2} \mathrm{~V}^{-1} \mathrm{~s}^{-1}$ (electrons) and $\approx 172 \mathrm{~cm}^{2} \mathrm{~V}^{-1} \mathrm{~s}^{-1}$ (holes). The EDL technique was also employed as an efficient tool to widely tune the range of Fermi level of BP. Saito and Iwasa demonstrated the mobilities of BP devices were $\approx 190$ and $\approx 20 \mathrm{~cm}^{2} \mathrm{~V}^{-1} \mathrm{~s}^{-1}$ (at $170 \mathrm{~K}$ ) for holes and electrons, respectively. ${ }^{[36]}$ Xiang et al. demonstrated that the SCTD of BP had an effective modulation toward ambipolar charge transport. For example, when BP surface was functionalized by molybdenum trioxide $\left(\mathrm{MoO}_{3}\right)$, the devices based on BP kept p-type behavior; when BP surface was functionalized by cesium carbonate $\left(\mathrm{Cs}_{2} \mathrm{CO}_{3}\right)$, the electrons mobility of black phosphorus was increased to $27 \mathrm{~cm}^{2} \mathrm{~V}^{-1} \mathrm{~s}^{-1}$. In this case, the devices based on BP presented ambipolar behaviors. ${ }^{[337]}$

As we all know, BP lacks of long-time ambient stability compared with TMDs. Therefore, researchers spend lots of time obtaining stable ambipolar devices. The most common method is the encapsulation technique. Yue et al. fabricated stable ambipolar transistors based on benzyl viologen-passivated BP and the devices exhibited large electron mobility as high as $\approx 83 \mathrm{~cm}^{2} \mathrm{~V}^{-1} \mathrm{~s}^{-1}$. ${ }^{338]} \mathrm{Zhu}$ et al. employed $\mathrm{Al}_{2} \mathrm{O}_{3}$ encapsulation layer to improve the long-time stability of the devices. They also manufactured flexible ambipolar transistors with high mobilities of about $310 \mathrm{~cm}^{2} \mathrm{~V}^{-1} \mathrm{~s}^{-1}$ (holes) and $89 \mathrm{~cm}^{2} \mathrm{~V}^{-1} \mathrm{~s}^{-1}$ (electrons). Another novel method is a marvelous defect-repair technique. Gui et al. realized excellent ambipolar performance via utilizing the aforementioned method. By exploiting hydrogenation and phosphorization, high mobilities of 237 and $177 \mathrm{~cm}^{2} \mathrm{~V}^{-1} \mathrm{~s}^{-1}$ were realized at $290 \mathrm{~K}$ for holes and electrons, respectively. ${ }^{[339]}$

\subsection{Van der Waals Heterostructures}

In parallel with the study of single-layer 2D materials, van der Waals heterostructures have also emerged as a new class of artificial material systems recently. These novel structures have revealed some new properties and special phenomena that could trigger new revolution of architecture design of heterostructures for modern optic, electronic and optoelectronic applications. ${ }^{[340-343]}$ For example, unlike electronic devices based on single-layer 2D materials where graphene is restricted because of its zero bandgap, vdW heterostructures provide an important opportunity to implement graphene-based electronic devices. ${ }^{[341,343]}$ With atomically thin molybdenum disulfide or boron nitride serving as a vertical transport barrier, transistors based on graphene heterostructures showed ambipolar behaviors with on/off current 
ratio of $\approx 50$ and $\approx 10^{4}$ for holes and electrons, respectively (Figure 21a). ${ }^{[341]}$ Fantastically, when a graphene gate was utilized, the transistor displayed large insulating window near the overall charge neutrality point (CNP) because of a longrange moiré superlattice potential and the broken local carbon sublattice symmetry in graphene (Figure 21b). ${ }^{[343]}$ In addition, $\mathrm{Gr} / \mathrm{WS}_{2} / \mathrm{Gr}$ heterostructures were introduced to expand the range of photoactive transistors for photoresponsivity application. Following these successes, the vertical integration of $\mathrm{p}-\mathrm{n}$ heterostructures based on other layered TMD semiconductors is also reported.

The $\mathrm{MoS}_{2}-\mathrm{WS}_{2}$ heterostructures with different configurations (vertical and planar structures, as shown in Figure 21c, d) were reported to possess new functionalities and superior (a)

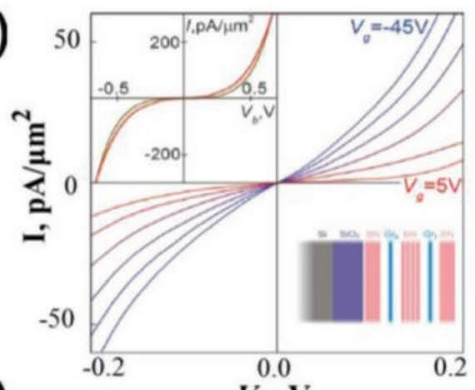

(c)

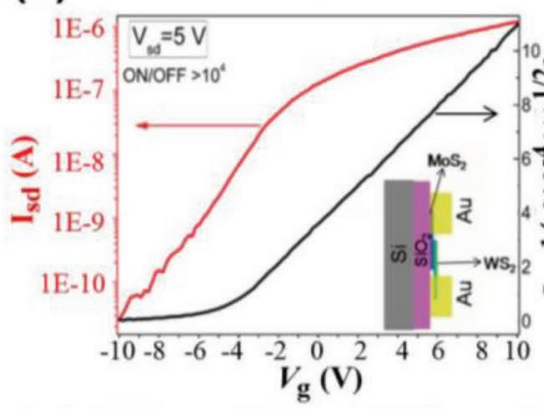

(e)
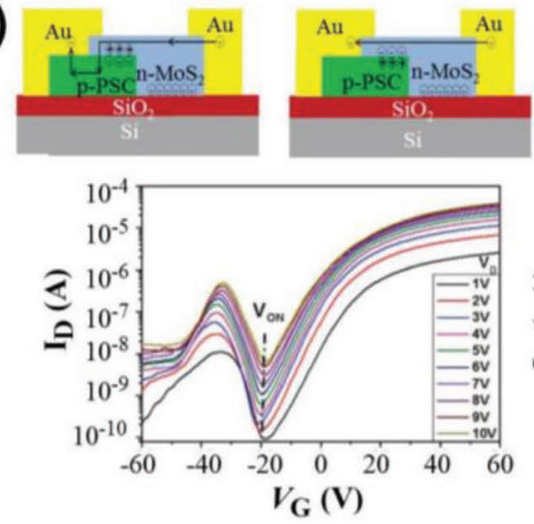

(b)

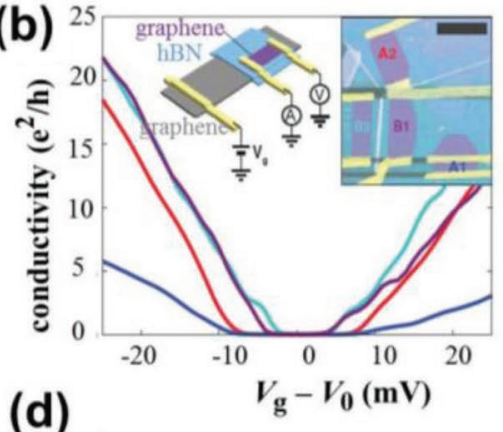

(d)

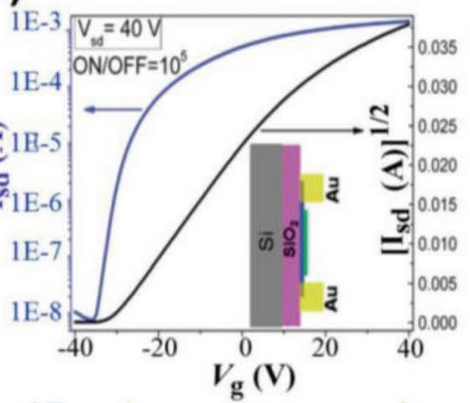

(f)
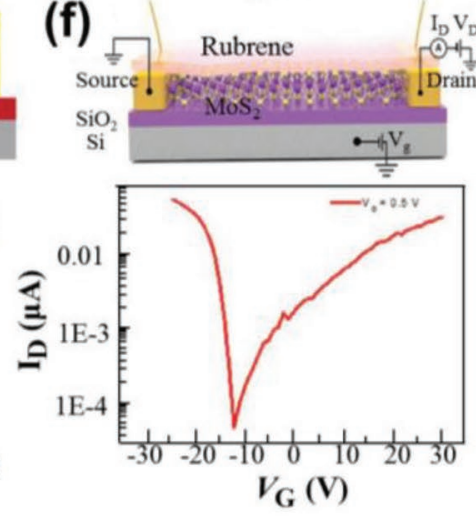

(g)

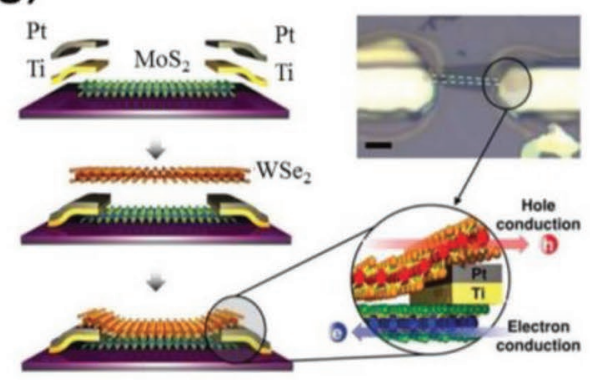

(h)

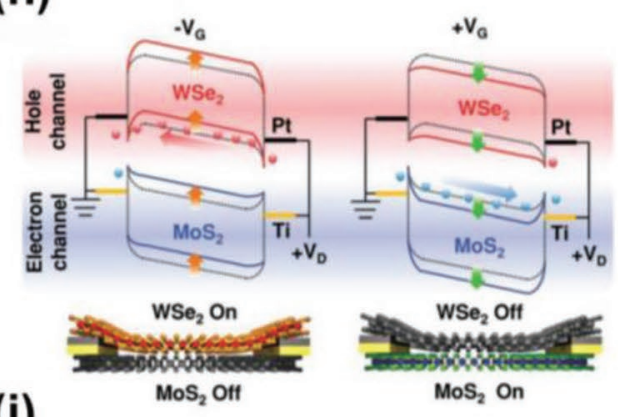

(i)

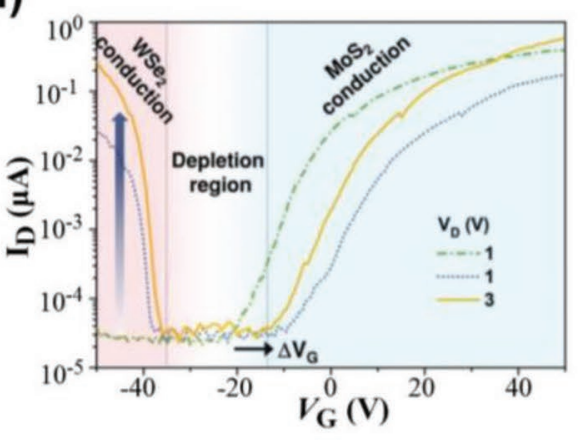

Figure 21. Schematic structures and $I-V$ transfer characteristics of transistors based on vdW heterostructures. a) $I-V_{\mathrm{s}}$ characteristics of a graphenehBN device for different $V_{\mathrm{g}}$ (in $10 \mathrm{~V}$ steps); the upper left inset: comparison of $I-V$ characteristics for the experimental (red curve) and theory (dark); the lower right inset: the schematic structure of a graphene field-effect tunneling transistor. Reproduced with permission. ${ }^{[31]}$ Copyright 2012, The American Association for the Advancement of Science. b) Low-temperature $(T=150 \mathrm{mK})$ conductivity curves of four heterostructure devices (A1, A2, B1, and B2); left inset: measurement schematic; right inset: false-color atomic force microscopy image. Reproduced with permission. ${ }^{[33]}$ Copyright 2013 , The American Association for the Advancement of Science. c) Transfer characteristics of the vertical transistors based on $\mathrm{MoS}_{2}-\mathrm{WS} \mathrm{S}_{2}$ heterostructures in the case of on-state; inset: the schematic diagram of the vertical device. d) Transfer characteristics of the planar transistor based on the $\mathrm{MoS}_{2}-\mathrm{WS}_{2}$ heterostructures; inset: the schematic diagram of the planar device. Reproduced with permission. ${ }^{[344]}$ Copyright 2014, Wiley- $\mathrm{VCH}$. e) The carrier transport schematic (upper left: the condition in $V>0$ and $V<0$; upper right: the condition in $V>>V_{T}$ ) and the transfer curve of Device II based on PSC/ $\mathrm{MoS}_{2}$ heterostructures. Reproduced with permission. ${ }^{[349]}$ Copyright 2017, the Royal Society of Chemistry. $f$ ) The schematic structure and the transfer characteristic of the ambipolar transistor based on the $\mathrm{MoS}_{2}$-rubrene heterostructure. Reproduced with permission. ${ }^{[350]} \mathrm{Copyright} 2017$, Wiley-VCH. g) Schematic of fabrication process for a dual-channel FET with $\mathrm{WSe}_{2} / \mathrm{MoS}_{2}$ heterostructure; the optical image of the FET device; illustration shows hole and electron transport in the $\mathrm{WSe}_{2}$ and $\mathrm{MoS}_{2}$ channels, respectively. h) Lateral band diagrams and lateral schematic structure of the dual-channel $\mathrm{WSe}_{2} / \mathrm{MoS}_{2} \mathrm{FET}$ at negative and positive $\mathrm{V}_{\mathrm{G}}$; black dotted line: the band structure of the $\mathrm{WSe}_{2} / \mathrm{MoS}_{2}$ stack; black solid line: Fermi levels of the Pt electrode; yellow solid line: Fermi levels of the Ti electrode; red solid line: the shift of CB and VB energy levels for WSe $\mathrm{W}_{2}$; blue solid line: the shift of CB and $V B$ energy levels for $M_{2} S_{2}$. i) $I_{D}-V_{C}$ curves of single-channel $M_{0} S_{2}$ FET before the transfer of WSe layer (green: $V_{D}=1 \mathrm{~V}$ ) and dual-channel WSe $\mathrm{W}_{2}$ $\mathrm{MoS}_{2}$ FET (blue: $V_{\mathrm{D}}=1 \mathrm{~V}$, yellow: $V_{\mathrm{D}}=3 \mathrm{~V}$ ). Reproduced with permission. ${ }^{[354]}$ Copyright 2016, Wiley-VCH. 
electronic and optoelectronic properties. These transistors exhibited novel rectifying behaviors (vertical structure), ambipolar behaviors (planar structure) as well as photovoltaic and photo-switching properties. ${ }^{[344]}$ The vertical device presented a high on/off current ratio $\left(>10^{4}\right)$ and a high electron mobility $\left(14 \mathrm{~cm}^{2} \mathrm{~V}^{-1} \mathrm{~s}^{-1}\right)$. The planar device also showed a high on/off current ratio $\left(10^{5}\right)$ and a high electron mobility $\left(65 \mathrm{~cm}^{2} \mathrm{~V}^{-1} \mathrm{~s}^{-1}\right)$. The similar electronic and optoelectronic properties as well as fantastic physical phenomena were also observed from the transistors based on $\mathrm{WSe}_{2} / \mathrm{WS}_{2}$, $\mathrm{ReSe}_{2} / \mathrm{WS}_{2}$, and $\mathrm{GaTe} / \mathrm{MoS}_{2}$ heterostructures. ${ }^{[345-347]}$ These heterostructures were unavoidable to be stacked with the mechanically exfoliated TMD layered semiconductors by a simple dry transfer procedure. Compared with mechanically exfoliated method, direct CVD approach to fabricate vertical heterostructures by vdW epitaxy can avoid interface contamination and result in well-defined interlayer. Li et al. utilized two-step CVD method to stack GaSe/MoSe 2 heterostructures directly and realized ambipolar transistors with tunable hole and electron charge transport. ${ }^{[348]}$

In addition to stacking different 2D layered materials, vdW heterostructures can also be achieved by combining 2D layered materials with organic semiconductors because organic semiconductors can interact with $2 \mathrm{D}$ materials via van der Waals force. The extension of the definition of vdW heterostructures reveals a wider research field and provides a chance to utilize various organic semiconductors. Dong et al. successfully stacked pentacene/ $\mathrm{MoS}_{2}$ vdW heterostructures. ${ }^{[349]}$ The transistors based on this vdW structure showed antiambipolar behaviors (present three states or ternary signals (one off-state and two various on-states) utilizing only a single transistor) (Figure 21e). When pentacene was replaced by rubrene crystals, ambipolar carrier conduction was successfully observed because of the band alignment between $\mathrm{MoS}_{2}$ and rubrene (Figure 21f). ${ }^{[350]}$ The ambipolar transistors exhibited well-balanced electron and hole mobilities of 1.27 and $0.36 \mathrm{~cm}^{2} \mathrm{~V}^{-1} \mathrm{~s}^{-1}$, respectively. Polymer (PDVT-10) has also been employed to fabricate vdW heterostructures with 2D materials by Yan et al. ${ }^{[351]}$ It should be of great concern that super-smooth surface of 2D materials offers an unique opportunity to control molecular packing structures of organic semiconducting crystals. ${ }^{[352]}$ Pentacene crystals can form uniform packing structure ("lying-down") on the surface of 2D materials (e.g., h-BN). Park et al. demonstrated "lying-down" pentacene crystals on h-BN, which could achieve both hole and electron transport. ${ }^{[353]}$

The traditional sole-channel $\mathrm{p}-\mathrm{n}$ transistors were stacked with two metal electrodes serving as source and drain on the top of $\mathrm{p}-\mathrm{n}$ heterostructures. The carriers should be transported across the $\mathrm{p}-\mathrm{n}$ junction. Therefore, it is difficult to achieve excellent transport properties for both electrons and holes simultaneously. A novel dual-channel $\mathrm{MoS}_{2} / \mathrm{WSe}_{2}$ heterostructure transistor was proposed and achieved (Figure 21g). ${ }^{[354]}$ Based on dual-channel heterostructure, the transistor could realize parallel carrier transport. When carriers are parallelly transported, the carriers are forbidden to transport across the $\mathrm{p}-\mathrm{n}$ junction. In contrast, this transistor allowed independent electron and hole transport (e.g., electrons in $\mathrm{MoS}_{2}$ layer and holes in $\mathrm{WSe}_{2}$ layer (Figure $\left.21 \mathrm{~h}\right)$ ). The combination of multiple transports could generate quite exotic electronic and optoelectronic properties. The devices showed ambipolar behaviors with a high on/off current ratio $\left(\approx 10^{4}\right)$ and large carrier mobilities of 5.97 and $0.54 \mathrm{~cm}^{2} \mathrm{~V}^{-1} \mathrm{~s}^{-1}$ for electrons and holes, respectively (Figure 21i). Compared with ambipolar transistors based on sole 2D material, transistors based on vdW heterostructures show more wonderful and miraculous electrical behaviors. These results have attracted more and more researchers to develop novel device structures and suitable material combinations.

\subsection{Other 2D Layered Materials}

In addition to traditional layered 2D materials (e.g., graphene, TMDs, h-BN, and BP), 2D organic systems and 2D perovskites also attract lots of attention..$^{[35-358]}$ For example, 2D perovskites based on naphthalene diimide ammonium (NDIA) and lead halide ((NDIA) $\left.)_{4} \mathrm{~Pb}_{3} \mathrm{I}_{10}\right)$ were demonstrated to show drain voltage-dependent charge transport behaviors. ${ }^{[359]}$ Although both electrical performance and stability were improved, the charge mobility was still inferior.

2D organic systems can be divided into three types: 2D covalent organic frameworks (COFs), $\pi$-conjugated polymers, and $\pi$-d-conjugated coordination polymers (CPs). 2D COFs are covalently constructed from planar aromatic building blocks based on the principle of reticular chemistry. Sun et al. reported transistors based on a $2 \mathrm{D}$ COF which was grown on single-layer graphene. The aforementioned transistors presented ambipolar charge transport under relatively low modulating bias. ${ }^{[360]}$ As we all known, $\pi$-conjugated polymers are formed by covalent-bonded aromatic units. On the contrary, CPs are composed of metal ions and $\pi$-conjugated ligands. This novel combination is expected to show fantastic electrical properties and physical phenomena due to the hybrid $d$-orbits of transition metals and frontier orbits of $\pi$-conjugated ligands. Huang et al. prepared a copper coordination polymer thin film with high crystallinity. The copper coordination polymer $\mathrm{Cu}-\mathrm{BHT}$ (BHT= benzenehexathiol) consisted of $2 \mathrm{D}$ $\left[\mathrm{Cu}_{3}\left(\mathrm{C}_{6} \mathrm{~S}_{6}\right)\right]_{n}$ nanosheets. ${ }^{[361]}$ The $\mathrm{Cu}-\mathrm{BHT}$ transistor exhibited ambipolar charge transport behavior as well as extremely high mobilities. The mobilities are 99 and $116 \mathrm{~cm}^{2} \mathrm{~V}^{-1} \mathrm{~s}^{-1}$ for holes and electrons, respectively.

In conclusion, both 2D layered materials and vdW heterostructures display great application potential in ambipolar transistors due to their high on/off ratio and carrier mobilities, as summarized in Table 3. Numerous techniques such as EDL, SCTD, electrostatic doping (ED) and chemical doping (CD) can be utilized to achieve and modulate ambipolar behaviors. Among them, EDL is the most efficient and common technique while SCTD and electrostatic doping are both efficient and nondestructive. Moreover, work function of metal electrodes is one of the key factors for fabricating ambipolar transistors and thereby both source and drain electrodes should be selected carefully. Finally, the ambipolar behaviors and electronic performance of many transistors based on 2D materials are thickness-dependent. Therefore, appropriate layers are also of great importance for manufacturing ambipolar transistors. 
Table 3. The mobilities, on/off ratios, and fabrication techniques for some reported ambipolar transistors based on 2D materials, vdW heterostructures, OIHPMs, and organic-inorganic dual layer.

\begin{tabular}{|c|c|c|c|c|c|c|c|}
\hline \multirow[t]{2}{*}{ Classification } & \multirow[t]{2}{*}{ Material } & \multicolumn{2}{|c|}{ FET mobility $\left[\mathrm{cm}^{2} \mathrm{~V}^{-1} \mathrm{~s}^{-1}\right]$} & \multicolumn{2}{|c|}{ ON/OFF ratio } & \multirow[t]{2}{*}{ Techniques } & \multirow[t]{2}{*}{ Ref. } \\
\hline & & $\mu_{\mathrm{e}}$ & $\mu_{\mathrm{h}}$ & $n$ & $\mathrm{p}$ & & \\
\hline \multirow[t]{25}{*}{$2 \mathrm{D}$} & $\mathrm{MoS}_{2}(\mathrm{ME})$ & 44 & 86 & $>10^{2}$ & $>10^{2}$ & EDLT & [302] \\
\hline & $\mathrm{MoS}_{2}(\mathrm{ME})$ & $>60$ & - & $>10^{7}$ & $>10^{4}$ & EDLT & [304] \\
\hline & $\mathrm{MoS}_{2}(\mathrm{CVD})$ & $\approx 10$ & - & $>10^{4}$ & $>10^{4}$ & EDLT & [305] \\
\hline & $\mathrm{MoS}_{2}(\mathrm{ME})$ & 470 & 480 & $>10^{3}$ & $>10^{5}$ & SE & [301] \\
\hline & $\mathrm{MoS}_{2}(\mathrm{ME})$ & 6.1 & 19 & $\approx 10^{6}$ & $>10^{5}$ & EDLT & [300] \\
\hline & $\mathrm{MoTe}_{2}$ (ME) & 0.03 & 0.3 & $>10^{2}$ & $>10^{3}$ & - & [312] \\
\hline & $\mathrm{MoTe}_{2}$ (ME) & - & 6.4 & $\approx 10$ & $10^{5}$ & - & [315] \\
\hline & $\mathrm{MoTe}_{2}$ (ME) & 80 & 41 & $\approx 10^{5}$ & $\approx 10^{4}$ & $C D$ & [284] \\
\hline & $\mathrm{MoTe}_{2}$ (ME) & 0.1 & 30 & $\approx 10^{2}$ & $\approx 10^{4}$ & SCTD & [316] \\
\hline & $\mathrm{MoTe}_{2}$ (ME) & 3.7 & 0.2 & $\approx 10^{4}$ & $>10^{5}$ & ED & [314] \\
\hline & $\mathrm{MoSe}_{2}$ (CVD) & 65 & 9 & $\approx 10^{5}$ & $\approx 10^{5}$ & - & [317] \\
\hline & $\mathrm{MoSe}_{2}$ (MBE) & 0.05 & 0.28 & $\approx 10^{4}$ & $\approx 10^{2}$ & EDLT & [319] \\
\hline & $\mathrm{MoSe}_{2}$ (CVD) & 0.6 & 0.1 & $>10^{6}$ & $>10^{6}$ & EDLT & [320] \\
\hline & $\mathrm{WS}_{2}(\mathrm{ME})$ & 20 & 90 & $>10^{2}$ & $>10^{2}$ & EDLT & [323] \\
\hline & $W S_{2}(M E)$ & 44 & 43 & $>10^{5}$ & $>10^{5}$ & EDLT & [321] \\
\hline & $\mathrm{WSe}_{2}$ (ME) & 72 & 180 & - & - & EDLT & [325] \\
\hline & $\mathrm{WSe}_{2}(\mathrm{MBE})$ & 1 & 3 & - & - & EDLT & [327] \\
\hline & $\mathrm{WSe}_{2}$ (ME) & 12.12 & 4.77 & $>10^{5}$ & $>10^{5}$ & $\mathrm{FP}$ & [326] \\
\hline & $\mathrm{BP}(\mathrm{ME})$ & 186.5 & 170.5 & $\approx 10$ & $\approx 10^{2}$ & - & [334] \\
\hline & $\mathrm{BP}(\mathrm{ME})$ & $\approx 38$ & $\approx 172$ & $\approx 10^{4}$ & $\approx 10^{4}$ & - & [333] \\
\hline & $\mathrm{BP}(\mathrm{ME})$ & 20 & 190 & $>10^{3}$ & $>10^{3}$ & EDLT & [336] \\
\hline & BP (ME) & 27 & $\approx 200$ & $\approx 10^{3}$ & $\approx 10^{4}$ & SCTD & [337] \\
\hline & $\mathrm{BP}(\mathrm{ME})$ & 83 & - & $\approx 10^{2}$ & $\approx 10^{3}$ & SCTD & [338] \\
\hline & $\mathrm{BP}(\mathrm{ME})$ & 177 & 237 & $\approx 10^{4}$ & $\approx 10^{4}$ & $C D$ & [339] \\
\hline & $\mathrm{BP}(\mathrm{ME})$ & 89 & 310 & $>10^{2}$ & $>10^{3}$ & - & [332] \\
\hline 2D COFs & TFPy-PPDA & - & - & $\approx 10^{5}$ & $\approx 10^{6}$ & - & [360] \\
\hline $2 \mathrm{DCPs}$ & {$\left[\mathrm{Cu}_{3}\left(\mathrm{C}_{6} \mathrm{~S}_{6}\right)\right]_{n}$} & 116 & 99 & $\approx 10^{2}$ & $\approx 10^{2}$ & - & [361] \\
\hline 2D perovskite & $(\mathrm{NDIA}){ }_{4} \mathrm{~Pb}_{3} \mathrm{I}_{10}$ & $5.44 \times 10^{-3}$ & $5.83 \times 10^{-3}$ & $\approx 10^{3}$ & $\approx 10^{3}$ & - & [359] \\
\hline \multirow[t]{10}{*}{$v d W$ heterostructures } & $\mathrm{MoS}_{2}-\mathrm{WS}_{2}$ & 65 & - & $>10^{5}$ & $10^{4}$ & - & [344] \\
\hline & $\mathrm{WSe}_{2} / \mathrm{WS}_{2}$ & - & - & $10^{5}$ & $10^{5}$ & - & [345] \\
\hline & $\mathrm{ReSe}_{2} / \mathrm{WS}_{2}$ & 0.1 & 0.4 & $10^{2}$ & - & - & [346] \\
\hline & $\mathrm{GaTe} / \mathrm{MoS}_{2}$ & 3.3 & 1.8 & $>10^{2}$ & $>10$ & - & [347] \\
\hline & $\mathrm{GaSe} / \mathrm{MoSe}_{2}$ & - & - & $>10^{2}$ & $>10^{2}$ & - & [348] \\
\hline & $\mathrm{WSe}_{2} / \mathrm{MoS}_{2}$ & 5.97 & 0.54 & $\approx 10^{4}$ & $\approx 10^{4}$ & - & [354] \\
\hline & $\mathrm{Gr} / \mathrm{h}-\mathrm{BN} / \mathrm{Gr}$ & - & - & $\approx 10^{4}$ & $\approx 50$ & - & [341] \\
\hline & rubrene/MoS 2 & 1.27 & 0.36 & $>10^{2}$ & $>10^{3}$ & - & [350] \\
\hline & PDVT-10/MoS 2 & 2.45 & 0.3 & $\approx 10^{3}$ & $\approx 10^{3}$ & - & [351] \\
\hline & pentacene/h-BN & 0.26 & 0.2 & $>10^{4}$ & $>10^{4}$ & - & [353] \\
\hline \multirow[t]{3}{*}{ OIHPMs } & $\mathrm{CH}_{3} \mathrm{NH}_{3} \mathrm{Pbl}_{3-x} \mathrm{Cl}_{x}$ & 1.01 & 1.24 & $>10^{4}$ & $>10^{4}$ & - & [14] \\
\hline & $\mathrm{CH}_{3} \mathrm{NH}_{3} \mathrm{Pbl}_{3-x} \mathrm{Cl}_{x}$ & 1.0 & 1.3 & $\approx 10^{2}$ & $\approx 10$ & - & [390] \\
\hline & $\mathrm{Cs}_{x}\left(\mathrm{MA}_{0.17} \mathrm{FA}_{0.83}\right)_{1-x} \mathrm{~Pb}\left(\mathrm{Br}_{0.17} \mathrm{l}_{0.83}\right)_{3}$ & 2.39 & 2.02 & $>10^{4}$ & $>10^{4}$ & - & [391] \\
\hline
\end{tabular}


Table 3. Continued.

\begin{tabular}{|c|c|c|c|c|c|c|c|}
\hline \multirow[t]{2}{*}{ Classification } & \multirow[t]{2}{*}{ Material } & \multicolumn{2}{|c|}{ FET mobility $\left[\mathrm{cm}^{2} \mathrm{~V}^{-1} \mathrm{~s}^{-1}\right]$} & \multicolumn{2}{|c|}{ ON/OFF ratio } & \multirow[t]{2}{*}{ Techniques } & \multirow[t]{2}{*}{ Ref. } \\
\hline & & $\mu_{\mathrm{e}}$ & $\mu_{\mathrm{h}}$ & $\mathrm{n}$ & $\mathrm{p}$ & & \\
\hline \multirow[t]{7}{*}{ OIDL } & InOx/Cytop/pentacene & 1.1 & 0.1 & $\approx 10-10^{2}$ & $\approx 10-10^{2}$ & - & [400] \\
\hline & $\operatorname{lnOx} /$ FBT-Th4 $(1,4)$ & 1.5 & 1.1 & $1.2 \times 10^{2}$ & $3.8 \times 10^{2}$ & - & [396] \\
\hline & IZO/pentacene & 13.8 & 0.14 & - & - & - & [401] \\
\hline & $\mathrm{ZnO} / \mathrm{DA} /$ pentacene & 0.38 & 0.34 & $\approx 10^{4}$ & $\approx 10^{3}$ & - & [403] \\
\hline & ZnO/PTAA:diF-TESADT & $2-4.5$ & $1.6-2.4$ & $\approx 10^{4}$ & $\approx 10^{4}$ & - & [398] \\
\hline & ZTO/SWCNTs & 11.5 & 4.2 & $\approx 10^{2}$ & $\approx 10^{2}$ & - & [404] \\
\hline & IGZOs/C8-BTBT & 5.1 & 4.5 & $\approx 10^{3}$ & $\approx 10^{3}$ & - & [397] \\
\hline
\end{tabular}

ME: mechanically exfoliated; CD: chemical doping; ED: electrostatic doping; MBE: molecular beam epitaxy; FP: ferroelectric polymer; SE: substrate engineering.

\subsection{D Materials}

1D materials (e.g., nanowires, nanoribbons, and nanorods) have attracted lots of attention in the application of FETs due to the realization of spin-control and quantized conductance. ${ }^{[362,363]}$ However, few researches have been carried out to utilize 1D materials in ambipolar transistors up to now. Among 1D materials, core/shell heterostructure nanowires are more popular because their electrical performance is easily modulated by reason of their unique structures consisting of two independent transporting channels (core channel and shell channel).

Apparently, the crystallinity, morphology and thickness of shell are three key factors to modulate ambipolar behaviors. Zhao et al. found that the crystallinity and morphology of $\mathrm{Si}$ shell were crucial to transistors on the basis of $\mathrm{Ge} / \mathrm{Si}$ core/shell nanowires. ${ }^{[364]}$ When amorphous $\mathrm{Si}$ (a-Si) was exploited as shell, the transistors presented strong p-type behavior because Ge core dominated the primary transport. For comparison, when crystalline $\mathrm{Si}$ (c-Si) was used, the transistors exhibited strong n-type behavior because the primary charge transport was determined by Si shell (Figure 22a). After adjusting the morphology of Si shell, charge transfer could be slightly modulated because of the interruption of charge transfer along the shell (Figure 22b). They also demonstrated that core/shell heterostructure nanowires could be realized by ingenious design and synthesis. In addition, the thickness of the shell can be employed to modulate the electrical behaviors of transistors based on $\mathrm{GaAs} / \mathrm{InSb}$ core-shell nanowire. Rieger et al. demonstrated that the electrical performance of transistors based on $\mathrm{GaAs} / \mathrm{InSb}$ core-shell nanowire was determined by the energy band of InSb shell rather than GaAs core. ${ }^{[365]}$ They also discovered that Fermi level shift was induced when the thickness of InSb shell gradually changed.

1D materials not only are suitable to study the fundamental charge transport, but also provide good strategy for sophisticated integrated optoelectronic circuits. Though the independent effect of core and shell has been studied, the synergistic effects of these two compositions lack of deep discussion. For example, charges transfer at the interface of these two compositions is very important and considered as a key factor in photovoltaic cells. ${ }^{[366]}$ However, relevant researches are rarely reported in nanowire transistors. Thus, research about this field is urgently required to bring some novel insights for nanowire transistors.

\subsection{Colloidal Nanocrystal Thin Films}

Colloidal nanocrystals (CNs) have been applied to ambipolar transistors for many years due to their numerous advantages such as efficient charge transport, ${ }^{[367]}$ tunable electronic and optical properties and high-throughput fabrication by inkprinting technique ${ }^{[368]}$.

Kang et al. reported low-voltage ambipolar TFTs on the basis of PbSe nanocrystal and an ion-gel gate blocking dielectric, which displayed electron and hole mobilities of 0.4 and $0.02 \mathrm{~cm}^{2} \mathrm{~V}^{-1} \mathrm{~s}^{-1}$, respectively. ${ }^{[369]}$ Zhou et al. successfully tuned the ambipolar behaviors of transistors utilizing a self-assembled monolayer of Au NPs. ${ }^{[370]}$ Plenty of researches about CN-based ambipolar transistors were reported by Loi group..$^{[367,371-377]}$ Especially, they demonstrated that PbS CN was one of the promising candidates because of its high mobility. The high electron $\left(1.91 \mathrm{~cm}^{2} \mathrm{~V}^{-1} \mathrm{~s}^{-1}\right)$ and hole $\left(0.15 \mathrm{~cm}^{2} \mathrm{~V}^{-1} \mathrm{~s}^{-1}\right)$ mobilities were obtained via systematic optimization of both coupling ligand molecules and transistor architectures (utilization of appropriate metal electrodes and electric double-layer gate). ${ }^{[367]}$ In addition, many key factors such as interface modification layer, ${ }^{[375]}$ double gates, ${ }^{[376]}$ surface SAM molecules, ${ }^{[371]}$ adscititious mechanical strain ${ }^{[372]}$ and operation temperature ${ }^{[373]}$ were also studied by Loi group. For example, by introducing Cytop as interface modification layer into $\mathrm{PbS}$-based ambipolar transistors, lowdensity capture states could be achieved at the interface. ${ }^{[375]}$ Therefore, the electron and hole mobilities could be significantly increased to 0.2 and $8 \times 10^{-4} \mathrm{~cm}^{2} \mathrm{~V}^{-1} \mathrm{~s}^{-1}$, as shown in Figure 23a. Besides, introduction of double gates could realize superior control of the threshold shift and give rise to controllable polarities of PbS-based transistors (Figure 23b-d). ${ }^{[376]}$ Moreover, the ligands on the surface of CNs play a significant role in determining their properties and hence electrical performance. Thus, suitable surface SAM molecules could also influence ambipolar behaviors of transistors. ${ }^{[371]}$ Furthermore, the mechanical strain and operating temperature were also employed to modulate ambipolar behaviors of 

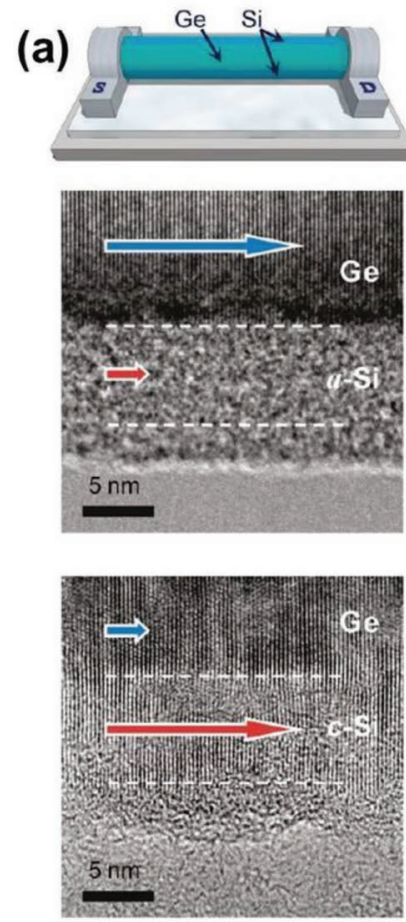
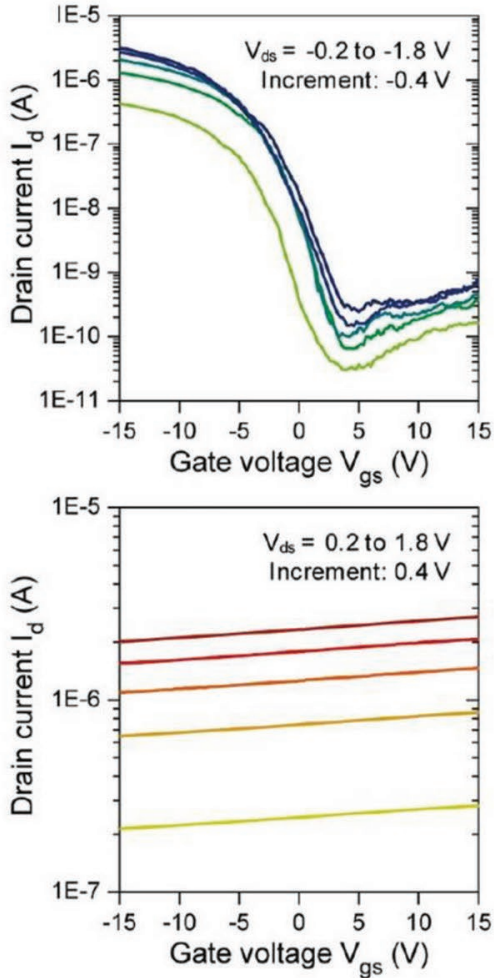

(b)
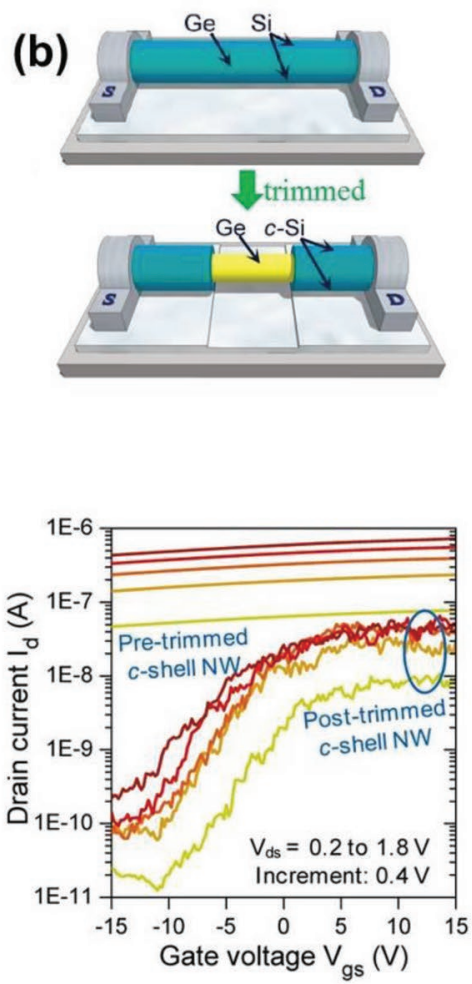

Figure 22. The influence of the crystallinity and morphology of Si shell on Ge/Si core/shell nanowire-based transistors. a) Schematic of the transistor (upper left), HRTEM image of the $\mathrm{n}$-type a-Si (middle left) and c-Si (lower left) shell nanowire; electrical characteristics of the n-type a-Si (upper right) and c-Si (lower right) shell nanowire transistors. b) Schematic of the trimmed n-type c-Si shell nanowire transistors (top) and their electrical characteristics (bottom). Reproduced with permission. ${ }^{[364]}$ Copyright 2011, American Chemical Society.

transistors. ${ }^{[372,373]}$ As shown in Figure 23e, low temperature led to symmetrical "V"-shaped ambipolar behaviors while high temperature resulted in asymmetrical ambipolar behaviors because of the attenuated hole transport. ${ }^{[373]}$ It was found that the temperature could significantly influence the hole trapping in $\mathrm{PbS}$ CNs. Other materials such as $\mathrm{FeIn}_{2} \mathrm{~S}_{4} \mathrm{CNs}$ and $\mathrm{PbSe}$ nanorod were also applied in transistors to study their ambipolar performance. ${ }^{[374,378]}$

In conclusion, CNs are a kind of critical ambipolar materials and many approaches have been utilized to control their ambipolar behaviors. In addition, some new methods (e.g., determinating energy levels of CNs) are utilized to realize ambipolar behaviors. ${ }^{[37]}$ Furthermore, a quite interesting ambipolar photoresponse is also observed in transistors based on CNs and 1D materials. ${ }^{[379,380]}$ Challenges still remain in achieving ambipolar carrier conduction utilizing other kinds of CNs.

\section{Organic-Inorganic Hybrid Materials}

\subsection{Organic-Inorganic Hybrid Perovskite Materials}

Organic-inorganic hybrid perovskite materials (OIHPMs) have been intensively pursued as a fantastic material systems due to a broad range of potential applications in multiple fields such as solar cells, ${ }^{[381,382]}$ sensors, ${ }^{[383]}$ ferroelectrics, ${ }^{[384]}$ memory devices, ${ }^{[385,386]}$ transistors, and thermoelectrics. ${ }^{[387-389]}$
Organic-inorganic hybrid materials have advantages of good processability of organic materials and excellent carrier mobilities of inorganic semiconductors. Transistors based on solution-processed organic-inorganic hybrid perovskite $\left(\mathrm{C}_{6} \mathrm{H}_{5} \mathrm{C}_{2} \mathrm{H}_{4} \mathrm{NH}_{3}\right)_{2} \mathrm{SnI}_{4}$ thin films displayed a high on/off current ratio of $10^{6}$ and a carrier mobility of $0.55 \mathrm{~cm}^{2} \mathrm{~V}^{-1} \mathrm{~s}^{-1}$. 388$]$ This result demonstrated that OIHPMs possessed bright progress toward electronic application. When $\mathrm{CH}_{3} \mathrm{NH}_{3} \mathrm{PbI}_{3}$ served as semiconducting layer, hole mobility of the devices could be increased to $3.55 \mathrm{~cm}^{2} \mathrm{~V}^{-1} \mathrm{~s}^{-1}$. After inserting $\mathrm{MoO}_{3}$ buffer layer, hole mobility of the transistors could reach up to $7.47 \mathrm{~cm}^{2} \mathrm{~V}^{-1} \mathrm{~s}^{-1} \cdot{ }^{[389]}$ Therefore, OIHPMs based on lead halide attract more and more attention and are extensively employed in ambipolar transistors. Besides, OIHPMs on the basis of lead halide can be easily synthesized from two common components (organic-amine halide and lead halide). For example, the well-known and representative material $\mathrm{CH}_{3} \mathrm{NH}_{3} \mathrm{PbI}_{3}$ $\left(\mathrm{MAPbI}_{3}\right)$ is formed by heating $\mathrm{PbI}_{2}$ and $\mathrm{CH}_{3} \mathrm{NH}_{3} \mathrm{I}$ mixed films. These semiconductors possess a favorable direct bandgap of 1.5-1.65 eV and a good absorption coefficient. Thus, OIHPMs are extremely promising to be utilized in ambipolar transistors. ${ }^{[14,359,390,391]}$

Li et al. fabricated a bottom-gate top-contact phototransistor on the basis of hybrid perovskite $\mathrm{CH}_{3} \mathrm{NH}_{3} \mathrm{PbI}_{3}$, which displayed ambipolar characteristics with superior carrier transport properties. ${ }^{[14]}$ The carrier transport property could be significantly improved by forming mixed-halide perovskites and 
(a)

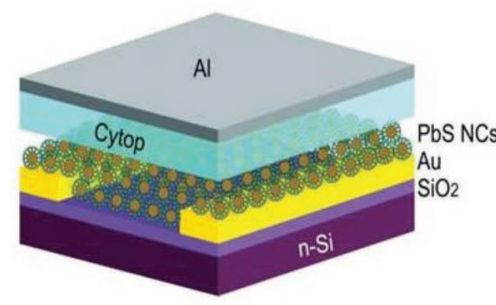

(b)
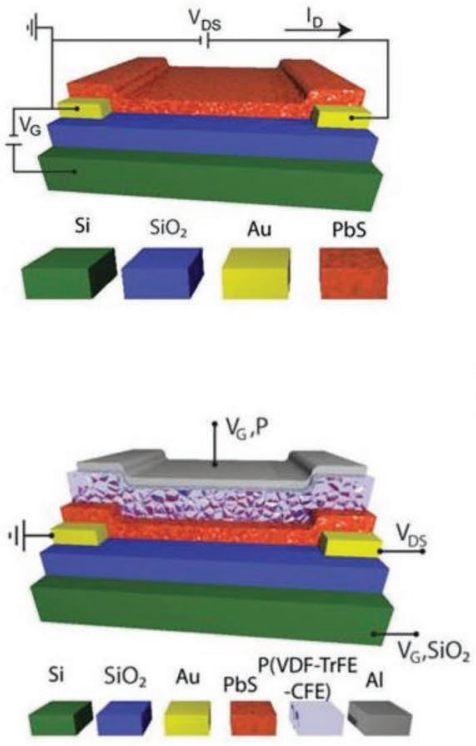

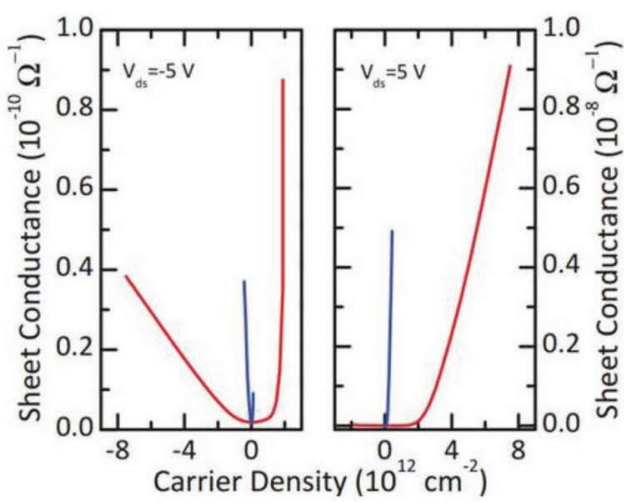

(e)

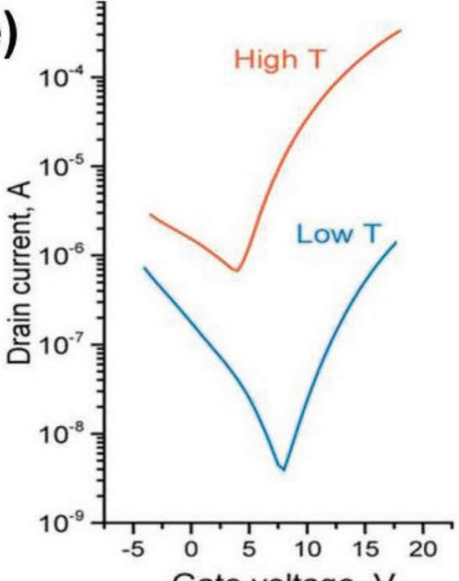

Gate voltage, V

(c)

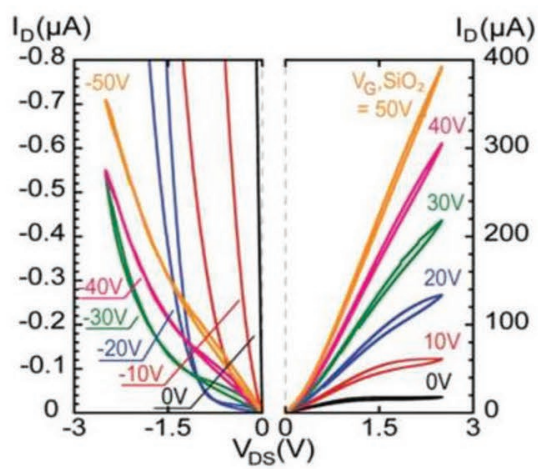

(d)

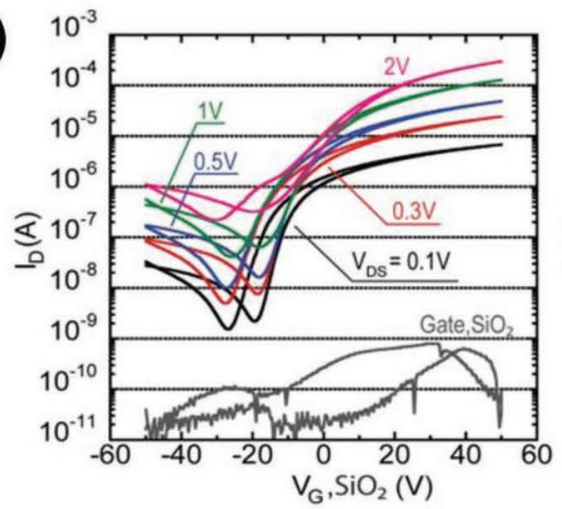

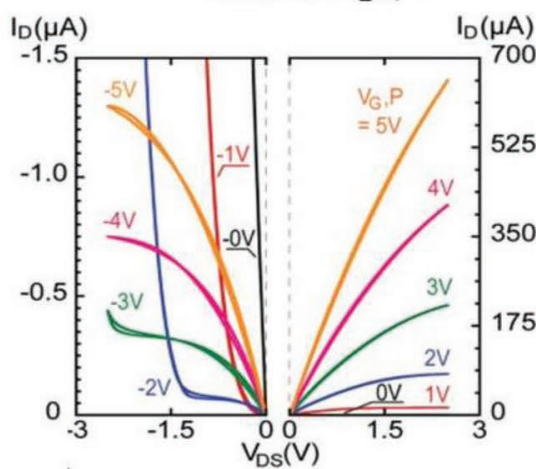

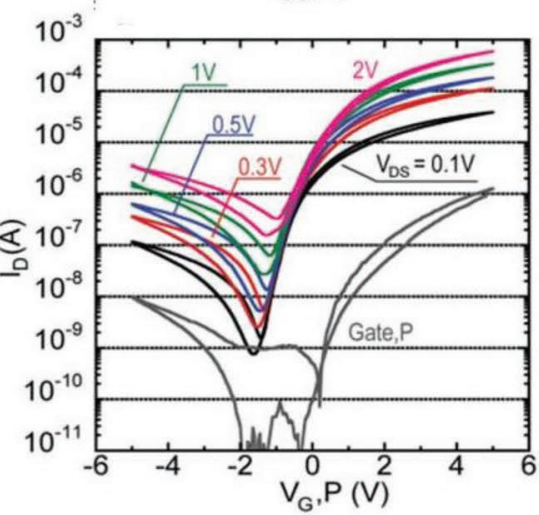

Figure 23. a) Schematic of PbS-based transistors using Cytop as interface modification layer (left); the comparison of sheet conductivity versus carrier density in PbS-based FETs without (red curve) and with Cytop (blue curve) interface modification layer (right). Reproduced with permission. ${ }^{375]}$ Copyright 2015, Wiley-VCH. b) Schematic structure of the single (top) and double (bottom) gate PbS-based transistor on $\mathrm{SiO}_{2} / \mathrm{Si}_{\text {substrate. c) Output }}$ characteristics of double-gate PbS-based transistors measured only connecting one gate: the $\mathrm{SiO}_{2}$ gate (left) or the $\mathrm{P}(\mathrm{VDF}-\mathrm{TrFE}-\mathrm{CFE})$ gate (right). d) Transfer characteristics of double-gate PbS transistors measured on the $\mathrm{SiO}_{2}$ gate (left) and the P(VDF-TrFE-CFE) gate (right, marked as Gate, P), respectively. Reproduced with permission. ${ }^{[376]}$ Copyright 2016, Wiley- $\mathrm{VCH}$. e) Transfer characteristics of the PbS transistor at high (180 K) and low temperature (80 K). Reproduced with permission. ${ }^{[373]}$ Copyright 2018, American Chemical Society.

introducing chlorine atoms instead of some iodine atoms. The balanced mobilities of mixed-halide perovskites could be increased to 1.01 and $1.24 \mathrm{~cm}^{2} \mathrm{~V}^{-1} \mathrm{~s}^{-1}$ for electrons and holes, respectively. The on/off current ratio was $10^{4}$ for both electron and hole transport. A similar phenomenon about augmented carrier mobility in mixed-halide perovskites was reported by Mei et al. ${ }^{[390]}$ These two works mentioned above demonstrated that increasing the grain size of perovskites and improving the interface quality between perovskites and blocking dielectric both could reduce charge carrier scattering and result in high mobility.

Formamidinium lead iodide $\left(\mathrm{FAPbI}_{3}\right)$ is another interesting and representative material of OIHPMs and has been widely studied in solar cells and photodetectors on account of its increasing absorption in the red light range. ${ }^{[392,393]}$ Nevertheless, its $\alpha$-phase structure is not stable and it can convert into a stable $\delta$-phase structure (yellow hexagonal phase) slowly, which has adverse effects on the electronic performance and optical 
absorption of $\mathrm{FAPbI}_{3}$. Fortunately, inorganic cesium (Cs) cation is suitable to improve the thermal and structural stability of perovskites. Yusoff et al. introduced Cs cations into crystal lattice of $\mathrm{FAPbI}_{3}$ to hamper the progress of $\delta$-phase impurities. They employed triple cation $\left(\mathrm{Cs}^{+}, \mathrm{MA}^{+}\right.$, and $\left.\mathrm{FA}^{+}\right)$perovskite to achieve defect-free, structurally and thermally stable as well as highly uniform perovskite films with a favorable bandgap $(1.6 \mathrm{eV}) .{ }^{[391]}$ Transistors based on these films presented unambiguous ambipolar characteristics with high on/off current ratio of $10^{4}$ and balanced mobilities of 2.39 and $2.02 \mathrm{~cm}^{2} \mathrm{~V}^{-1} \mathrm{~s}^{-1}$ for electrons and holes, respectively.

As mentioned above, OIHPMs have been successfully employed to fabricate transistors with excellent ambipolar characteristics and novel physical phenomena. The balanced mobilities have exceeded $2 \mathrm{~cm}^{2} \mathrm{~V}^{-1} \mathrm{~s}^{-1}$ for both electrons and holes (Table 3). The component of OIHPMs, the perovskite grain size and the interface quality between perovskites and blocking dielectric are both key factors for achieving high and balanced carrier mobilities. Moreover, these precursory researches will open new pathway to understand the fundamental performance of OIHPMs and expand the applications of OIHPMs in electronic devices.

\subsection{Organic-Inorganic Bilayer}

Although tremendous efforts have been paid for the improvement of both hole and electron mobilities of ambipolar transistors based on single-component semiconducting materials, electron and hole transport are not well balanced for most of the time. ${ }^{[394,395]}$ For example, organic semiconductors can transport holes and electrons simultaneously. However, for most of organic semiconductors, electron transport is poor compared to hole transport. On the contrary, most inorganic semiconductors exhibit only n-type characteristic with high electron mobility. Therefore, organic-inorganic bilayer could be another method to combine advantages of organic and inorganic materials and realize superior carrier mobilities for both electrons and holes. ${ }^{\text {396-399] }}$

The organic-inorganic bilayer ambipolar transistors have been reported in many organic-inorganic hybrid systems (e.g., combination of $\mathrm{ZnO}, \mathrm{In}_{2} \mathrm{O}_{3}, \mathrm{SnO}_{2}$, and p-type organic materials). The most utilized organic material in hybrid systems is pentacene due to its good crystallinity and high hole mobility. Besides, low-bandgap donor-acceptor or $\pi-\pi$ packing conjugated polymers (e.g., FBT-Th $4(1,4)$ and poly(3-hexylthiophene)), $\pi-\pi$ packing small molecules (e.g., dinaphtho-thienothiophene and dioctylbenzothieno[2,3-b]benzothiophene (C8-BTBT)) and organic blends (e.g., PTAA:diF-TESAD) were also studied as an organic layer in bilayer ambipolar transistors.

The transistors on the basis of $\operatorname{In}_{2} \mathrm{O}_{3}$ and indium zinc oxide (IZO) present typical n-type operation while the transistors based on pentacene single layer exhibit representative p-type operation. Sun et al. reported that the utilization of $\operatorname{In}_{2} \mathrm{O}_{3}$, IZO and pentacene as active channel could give rise to ambipolar behaviors. ${ }^{[400]}$ The mobilities of the devices were 1.1 and $0.1 \mathrm{~cm}^{2} \mathrm{~V}^{-1} \mathrm{~s}^{-1}$ for electrons and holes, respectively. Based on further Zn-doping, the electron mobility of IZO/pentacene devices was significantly enhanced and reached up to
$13.8 \mathrm{~cm}^{2} \mathrm{~V}^{-1} \mathrm{~s}^{-1}$ while the hole mobility of the aforementioned devices was still as low as $0.14 \mathrm{~cm}^{2} \mathrm{~V}^{-1} \mathrm{~s}^{-1}$. ${ }^{[401]}$ Although ambipolar charge transport was successfully realized, the mobilities of electrons and holes were unbalanced and the mobility of electrons was ten times greater than that of holes. After introducing low-bandgap donor-acceptor-conjugated polymer $\left(\right.$ FBT-Th $\left._{4}(1,4)\right), \mathrm{In}_{2} \mathrm{O}_{3}$-based transistors displayed high and wellbalanced mobilities of 1.5 and $1.1 \mathrm{~cm}^{2} \mathrm{~V}^{-1} \mathrm{~s}^{-1}$ for electrons and holes, respectively. ${ }^{[396]}$ However, the device configuration was relatively complicated.

Compared with ambipolar transistors on the basis of $\operatorname{In}_{2} \mathrm{O}_{3}$ and IZO, ZnO-based transistors exhibit more balanced characteristics when $\mathrm{ZnO}$ is combined with pentacene and P3HT, as listed in Table 3. For example, Zhou et al. reported flexible ambipolar transistors based on solution-processed $\mathrm{ZnO}$ and P3HT heterojunction. ${ }^{[402]}$ Although the devices presented balanced carrier mobilities, the hole and electron mobilities were inferior. When P3HT was replaced by pentacene, the ambipolar transistors displayed increased mobilities $\left(0.38\right.$ and $0.34 \mathrm{~cm}^{2} \mathrm{~V}^{-1} \mathrm{~s}^{-1}$ for electrons and holes, respectively). ${ }^{[403]}$ In order to improve the mobilities of both electrons and holes, the organic blends (PTAA:diF-TESAD) were employed to be in combination with ZnO layer. As a result, ambipolar transistors showed extremely large carrier mobilities exceeding $2 \mathrm{~cm}^{2} \mathrm{~V}^{-1} \mathrm{~s}^{-1}$. ${ }^{[398]}$ Moreover, ambipolar transistors on the basis of $\mathrm{ZnO}$ and PTAA:diFTESAD blends could also be exploited for the realization of unipolar high-mobility devices via the voltage modulation of top and bottom gates. In addition to organic materials, singlewalled carbon nanotubes (SWCNTs) were also employed to combine with $\mathrm{ZnO}$ in ambipolar transistors due to their high mobilities (larger than $30 \mathrm{~cm}^{2} \mathrm{~V}^{-1} \mathrm{~s}^{-1}$ ) and a large on/off current ratio $\left(>10^{5}\right) \cdot{ }^{[404]}$ As expected, ambipolar transistors based on ZTO(zinc tin oxide)/SWCNTs bilayer showed superior environmental stability and high mobilities of 11.5 and $4.2 \mathrm{~cm}^{2} \mathrm{~V}^{-1} \mathrm{~s}^{-1}$ for electrons and holes, respectively. The enhancement of hole transport presumably resulted from atmospheric adsorbate doping effects of SWCNTs. These results indicate that doping of metal oxides as well as appropriate combination and introduction of organic blends play a critical role in the excellent electrical performance of organic-inorganic bilayer ambipolar transistors.

Amorphous indium-gallium-zinc oxides (a-IGZOs) are excellent $n$-type semiconductors with large mobility of more than $10 \mathrm{~cm}^{2} \mathrm{~V}^{-1} \mathrm{~s}^{-1}$ and have been widely applied in transistors due to their high reliability and uniformity, large-area fabrication and mechanical flexibility. ${ }^{[405]} \mathrm{Li}$ et al. combined a-IGZOs with organic small molecule DNTT or C8-BTBT to fabricate ambipolar transistors. ${ }^{[397]}$ The transistors based on a-IGZOs/DNTT exhibited excellent electrical performance with mobilities as large as 5.1 and $2.8 \mathrm{~cm}^{2} \mathrm{~V}^{-1} \mathrm{~s}^{-1}$ for electrons and holes, respectively. When a-IGZOs were in combination with C8-BTBT, balanced mobilities as high as 5.1 and $4.5 \mathrm{~cm}^{2} \mathrm{~V}^{-1} \mathrm{~s}^{-1}$ for electrons and holes could be achieved, which originated from charge injection in the $\mathrm{p}-\mathrm{n}$ heterojunction.

On the contrary to traditional n-type metal oxides and p-type organic semiconducting hybrid systems, p-type metal oxides and n-type organic semiconducting hybrid heterojunctions were also investigated to realize ambipolar performance. Boratto et al. demonstrated ambipolar transistors based on 
p-type antimony-doped tin dioxide $\left(\mathrm{Sb}: \mathrm{SnO}_{2}\right)$ and n-type phenyl- $\mathrm{C}_{61}$-butyric acid methyl ester heterojunction. However, both hole and electron mobilities were inferior. ${ }^{[406]}$ In conclusion, organic-inorganic bilayer ambipolar transistors can take full advantages of the outstanding electrical performance of each component in the $\mathrm{p}-\mathrm{n}$ heterojunction. The well-balanced electron and hole mobilities can be successfully achieved by utilizing reasonable combination of n-type metal oxides and p-type organic semiconducting hybrid systems.

\section{Functional Applications of Ambipolar Transistors}

\subsection{Ambipolar Memory Transistors}

Transistor-type nonvolatile memories have received extensive attention on account of their nondestructive read-out, single transistor architecture and compatibility with complementary integrated circuits. ${ }^{[407-413]}$ One common class is floatinggate flash memory which consists of gate electrode, blocking dielectric (prevent charge transport between gate electrode and floating gate), floating gate (store charges), tunneling layer (determine program/erase speed and prevent leakage of trapped carriers), semiconducting active layer and source/ drain electrodes. By applying electrical bias with opposite voltage polarities to gate electrode, charge carriers can be stored into floating gate (program operation) or be released from floating gate into conducting channel (erase operation). During these processes, the electrical transfer curve of transistors will shift and the two different transfer curves (represent two various states: "0" state and "1" state) after program/erase operation can be utilized for storing binary data, which is the foundation of flash memories. One can also simply judge the polarities of the memory transistors on the basis of the shift directions of transfer curves in contrast to the initial state. Specifically, for ambipolar floating-gate flash memory, the transfer curve shifts to one side in regard to the initial state after program process and moves to the other side in comparison with the initial state after erase operation, which can be explained by the bipolar charge storing under reverse bias polarities (reverse vertical electrical fields). Obviously, ambipolar memories possess larger memory window due to the bidirectional threshold voltage shift and thereby easier realized multilevel data storage, which is urgently required for miniaturization of commercial memory devices to enhance memory capacity and density.

Compared to continuous planar floating gate, nanostructured materials (i.e., metal nanoparticles) are widely investigated due to manipulatable storage capability and storage sites. The metal nanofloating gate can be fabricated via thermal evaporation, self-assembly, microcontact printing, etc. Among them, gold $(\mathrm{Au})$ nanoparticles are more commonly employed for charge storage elements by reason of favorable chemical stability and large work function. Ambipolar behavior can be achieved by modulating the interface between diverse layers. Han et al. fabricated bipolar nonvolatile transistor memories with Au nanoparticles as charge storage elements, tetratetracontane as tunneling layer and pentacene as semiconducting layer, respectively. ${ }^{[414]}$ It was found that the smooth interface between tunneling and semiconducting layer suppressed the capture of electrons and led to ambipolar performance with large memory window $(18.1 \mathrm{eV})$ and good retention of $7.5 \mathrm{~h}$. Besides, various ambipolar semiconducting materials are known to easily realize ambipolar charge storage due to their intrinsic coexisted holes and electrons. ${ }^{[415,416]}$ Zhou et al. proposed a flexible multibit data storage flash memory which utilized solutionprocessing ambipolar conjugated polymer poly(diketopyrrolo pyrrolethiophenebenzothiadiazolethiophene) (PDPP-TBT) as semiconducting material to achieve ambipolar charge trapping in $\mathrm{Au}$ nanoparticles (Figure 24a). ${ }^{[417]}$ By adjusting the polarity and amplitude of gate and source-drain biases, large memory window and five distinct storage levels (multibit data storage) were realized (Figure 24b), signifying its promising applications in memory fields. Very recently, Chen et al. demonstrated all-inorganic flash memories based on the structure of Si gate/ $\mathrm{SiO}_{2} / \mathrm{Au}$ nanoparticles/ PMMA/cesium lead halide quantum dot/Au source-drain electrodes via a solution-processable layerby-layer assembling approach. ${ }^{[418]}$ The floating-gate memories exhibited large memory window of $2.4 \mathrm{~V}$, good data retention of more than $10^{5} \mathrm{~s}$ and stable endurance ( $\geq 200$ cycles) under the application of relatively small operation voltage of $\pm 5 \mathrm{~V}$. Although the metallic floating gates have many advantages, it is difficult to synthesize metal nanoparticles with homogenous size and spatial contribution directly and require complicated adjustment of fabrication parameters and large energy dissipation.

Another strategy to achieve ambipolar flash memories is introducing double floating gate (DFG) structures, which can prevent the leakage of the already trapped carriers due to the existed leakage barrier between two stacked floating gates induced by their band offset (improved retention capacity) and lead to better memory performance through ambipolar charge storage. ${ }^{[419]}$ However, it is still challenging to form vertical nanoparticle pairs and smooth interface between floating gates and tunneling layer. Thus, appropriate work function alignment (generate potential barrier), precise spatial distribution and flatten interface are necessary for constructing effective DFG architectures. A novel low-voltage nonvolatile OFET memory based on single-crystal $\mathrm{C}_{60}$ needles/copper phthalocyanine (CuPc) nanoparticles DFG on glass substrates has been developed by Chang et al. (Figure 24c). ${ }^{[16]}$ High- $k$ hafnium oxide was utilized as dielectric layer to achieve low operation voltage and high on/off current ratio. For DFG, discrete $\mathrm{C}_{60}$ needles (about $590 \mathrm{~nm}$ ) were first deposited for storing holes via a droplet crystallization approach after which discontinuous CuPc nanoparticles (about $15 \mathrm{~nm}$ ) were thermally evaporated for trapping electrons. These heterostructured floating gates were then covered by tunneling dielectric poly(4-vinylpheneol). In contrast to individual floating-gate flash memories (respect $\mathrm{C}_{60}$ or $\mathrm{CuPc}$ device), the DFG memories presented wider memory window $(4.4 \mathrm{~V})$, larger on/off current ratio $\left(8.3 \times 10^{3}\right)$, stable endurance property (up to 500 cycles) and good retention capability $\left(10^{4} \mathrm{~s}\right)$, signifying the electrical reliability of the DFG memory (Figure 24d).

Apart from aforementioned floating-gate flash memory, another important type of transistor memories is charge-trap flash memory which exploits dielectric materials with electret 
(a)
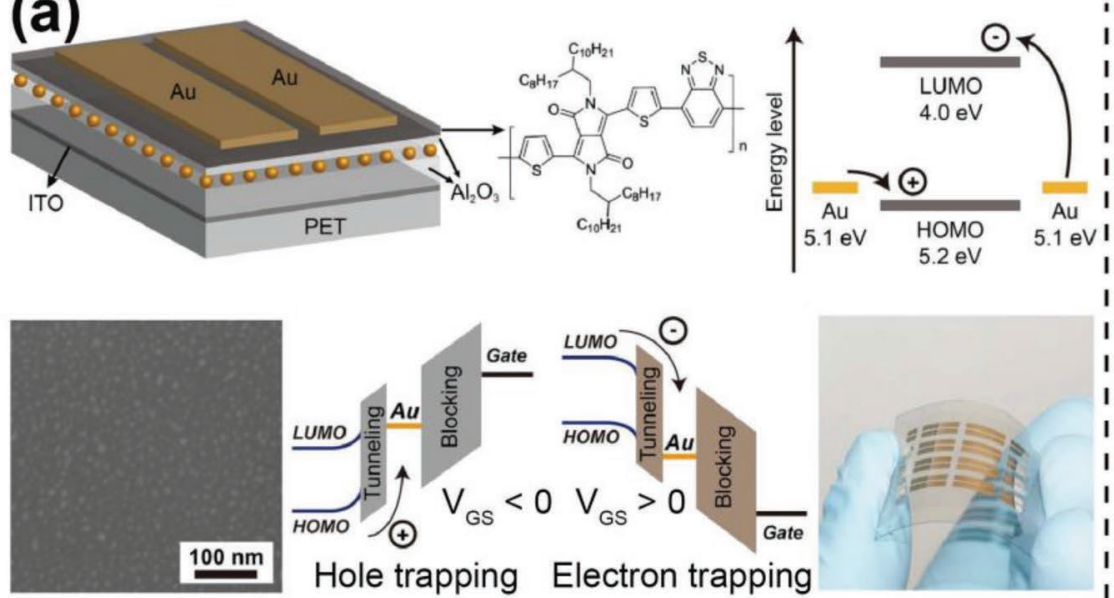

(b)
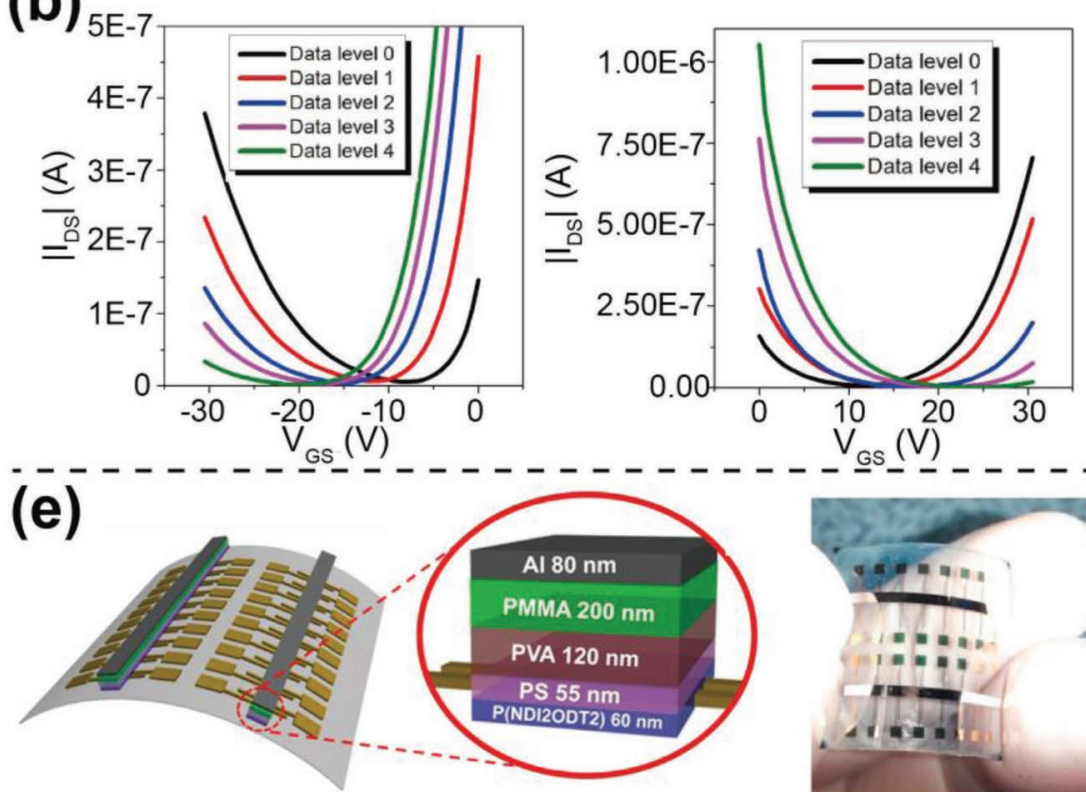

(g)
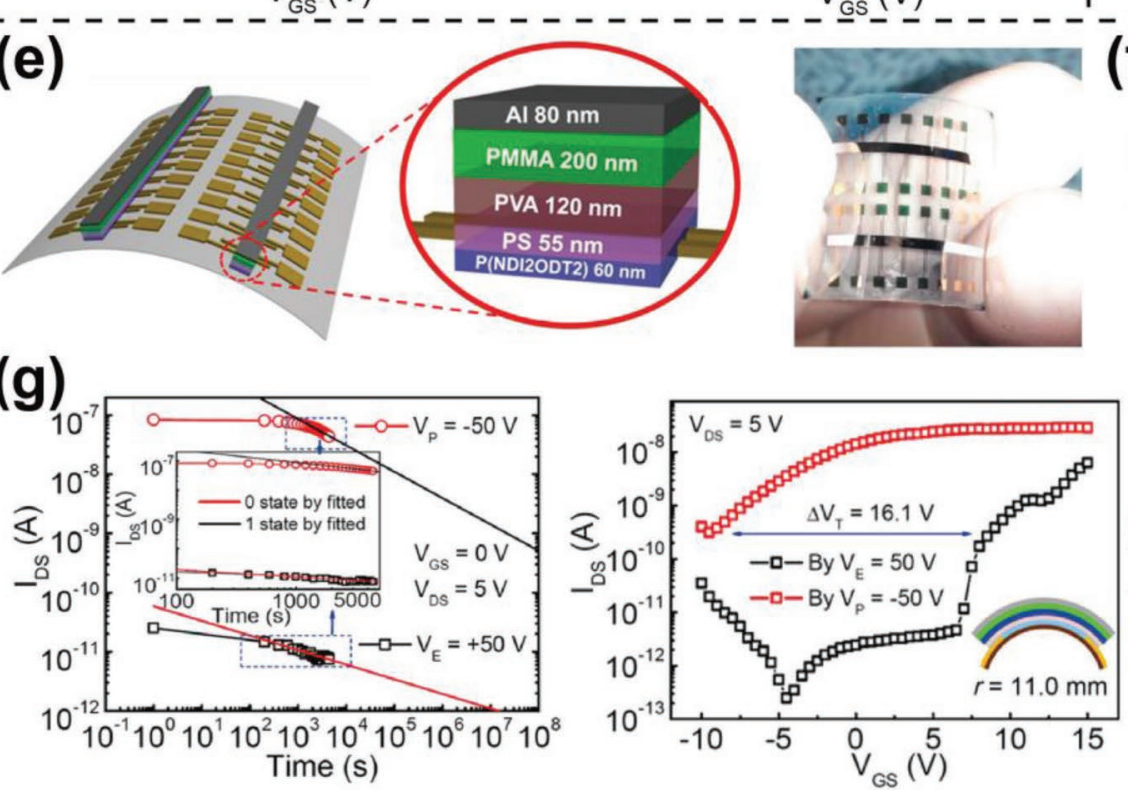

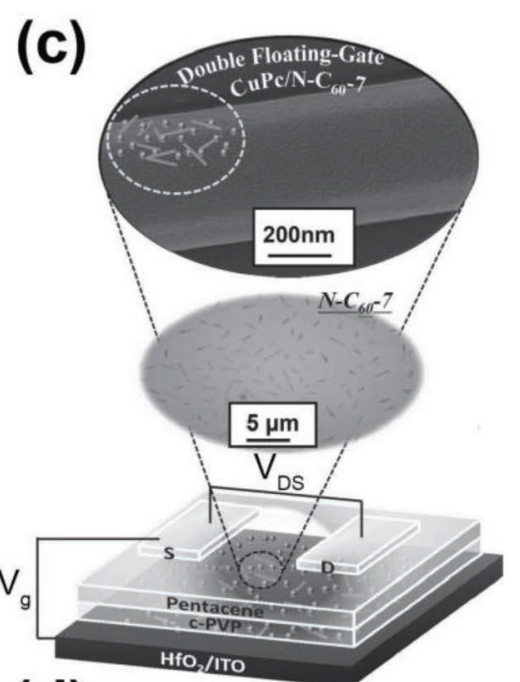

(d)

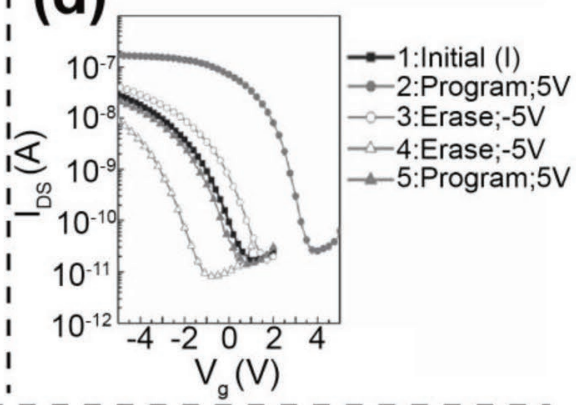

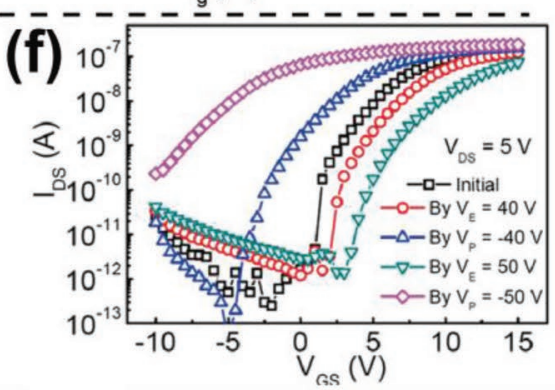

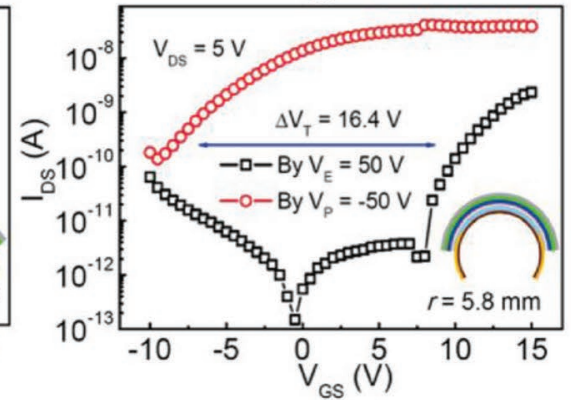

Figure 24. a) From left to right, from top to bottom: 3D schematic illustration of the ambipolar flash memory; molecular structure of organic material PDPP-TBT; energy band alignments between PDPP-TBT and source-drain gold electrodes; SEM morphology of Au nanoparticle charge trapping layer; hole and electron trapping mechanisms of the flash memory revealed by energy band structures; optical graph of the aforementioned flexible flash memory. b) Representative transfer characteristics of five various data levels of the memory transistor (left: hole-enhancement regime; right: electron-enhancement regime). Reproduced with permission. ${ }^{[417]}$ Copyright 2013, Nature Publishing Group. c) 3D OFET memory structure comprising $\mathrm{CuPc} / \mathrm{N}-\mathrm{C}_{60}$ DFG architecture and the optical microscopy and TEM morphologies of the DFG. d) Typical transfer curves of memory transistors based on $\mathrm{CuPc} / \mathrm{N}-\mathrm{C}_{60}$ DFG architecture. Reproduced with permission. ${ }^{[16]}$ Copyright 2015, Wiley-VCH. e) Schematic diagram (left) and image (right) of arrays of flexible memory transistors. f) Representative transfer curves of the flexible OTFT nonvolatile memories under repetitive program and erase operations. g) Retention capability of the flexible transistors under flat state and transfer curves of the flash memories under the tensile bending radius of $11.0 \mathrm{~mm}$ (left) and $5.8 \mathrm{~mm}$ (right), respectively. Reproduced with permission. ${ }^{[426]}$ Copyright 2015, American Chemical Society. 
characteristics to capture the charge carriers of the semiconducting channel. ${ }^{[420,421]}$

Alternatively, polymer electret is attractive for chargetrap flash memory as a result of facile and low-cost solutionprocessing uniform charge storage layer via spin-coating approach and tunable bandgap (controllable energy barrier of charge injection between charge storage layer and semiconducting active layer). ${ }^{[42-425]}$ Since the surface or interface of the polymer layer plays a significant role in capturing and releasing charge carriers, suitable orthogonal solvents of the polymer solution, appropriate film thickness, smooth layer morphology and interfaces between layers are highly demanded for highperformance flash memories. Wang et al. designed bottomcontact top-gated flexible transistor memories comprising four functional layers based on conjugated polymers onto bendable poly(ether sulfone) (PES) substrate via a successive spincoating method (Figure 24e). ${ }^{[26]}$ Poly(vinyl alcohol) (PVA) was utilized as charge storage elements and sandwiched between the interface of PMMA blocking layer and polystyrene (PS) tunneling dielectric. Air-stable P(NDI2OD-T2) was employed as semiconducting channel due to its high dissolvability, large charge mobilities and bipolar charge movement induced by coexisted donor and acceptor moieties. The authors discovered that both the selection of blocking polymers and the thickness of blocking and tunneling layers had an impact upon the electrical reliability of memories. By optimizing these parameters, the flash memory displayed large memory window (15.4 V) and high on/off current ratio $\left(2 \times 10^{4}\right)$ as well as excellent data retention capability $\left(10^{8} \mathrm{~s}\right)$, which exhibited no obvious degradation after repetitive bending operations (1000 times; bending radius of $5.8 \mathrm{~mm}$ ) (Figure $24 \mathrm{f}, \mathrm{g}$ ).

Graphene oxide (GO) is a product of oxidation of graphene and possesses abundant surface functional groups such as carbonyl, epoxy and hydroxylic groups, hence plentiful charge storage sites as well as high catalytic activity. ${ }^{[27]}$ Reduced graphene oxide ( $\mathrm{rGO}$ ) is reduced on the basis of GO and thereby loses the oxidized functional moieties. The transparent $\mathrm{GO}$ and $\mathrm{rGO}$ have been employed for realizing charge-trap flash memories. Han et al. put forward a novel strategy to exploit alkylsilane self-assembled monolayer to functionalize charge-capturing $\mathrm{rGO}$ sheets through a facile solution-processing approach and achieved successful modulation of the Fermi level of rGO within large scope (4.3-5.7 V), which was more advantageous compared to chemical-doped rGO. ${ }^{[28]}$ Electron-deficient (tridecafluoro-1,1,2,2-tetrahydrooctyl) trichlorosilane (FTS) and electron-rich aminopropyl triethoxysilane (APTES) were chosen to dope rGO sheets into p- and n-type charge-trapping layers and further induce various injection barriers between rGO layers and HOMO/LUMO levels of the semiconducting layer, which finally resulted in ambipolar or unipolar charge trapping performance. Besides, the tunable work functions of rGO layers could realize controllable threshold voltage shifts of the flash memories. By combining the functionalized rGO-based ambipolar memory and flexible plastic polymer substrate, the device exhibited stable and reliable low-voltage operation under multiple bending cycles, demonstrating the promising applications of flexible transparent electronics on the basis of SAM-doped rGO.
In addition to the above-mentioned bipolar flash memories programmed/erased by electrical stress, light irradiation has also presented to induce the threshold voltage shift of the transfer curve. Photo-operated ambipolar memories can be achieved on account of generation and separation of light-triggered excitons and subsequently augmenting trappable charge carriers. Besides, it is apparent that the large electrical power consumption can be significantly decreased by introducing optical stimulation. Recently, Yi et al. demonstrated the transition from unipolar memory to ambipolar memory via exerting white light illumination onto the semiconducting layers (pentacene/poly( $N$-vinylcarbazole) (PVK)) of organic transistors. ${ }^{[429]}$ It was found that both electrical bias and light irradiation were capable of realizing individual program/erase process for storing data. The lightilluminated ambipolar flash memories displayed stable electrical properties with good retention ability and large on/off current ratio, particularly, extremely large doubled memory window $(\approx 70 \mathrm{~V})$ compared to unipolar devices. Despite the effectiveness of light illumination for ambipolar memories, few studies are carried out and more investigations of this field are expected.

\subsection{Ambipolar Synaptic Transistors}

Human brain is a plastic and fault-tolerant complex computational system where approximately $10^{11}$ chemical neurons interlace with each other and develop into interwoven connected neural networks. Among them, $10^{14}$ neurological synapses are functional links and key elements for signal transmission in which the impulse of one neuron (presynaptic membrane) passes (synaptic cleft) to another neuron or to another cell (postsynaptic membrane). During this process, the biochemical information is processed, transmitted and stored at the same time via modulating the linkage strengths between various neurons (achieved by tuning the amounts of neurotransmitters and metal cations such as $\mathrm{Ca}^{2+}$ and $\mathrm{K}^{+}$). This biological synaptic plasticity is believed to settle the bottleneck of conventional von Neumann structure of computers and connect computational and memory units together. ${ }^{[430,431]}$

On this score, it is essential to investigate novel nanoscale plastic synaptic devices for efficient chemical synaptic emulation and further their integration circuits for neuromorphic computing. Mead first proposed the concept of neuromorphic engineering in 1990 and utilized silicon-based electronic circuits to simulate the neural networks. ${ }^{[40]}$ Thereafter, various mimicry technologies employing basic electronic elements for instance CMOS circuits, ${ }^{[432]}$ two-terminal memristors, ${ }^{[433,434]}$ resistive-switching, ${ }^{[435]}$ phase-change and ferroelectric memories, ${ }^{[436]}$ and three-terminal transistors ${ }^{[437]}$ have been implemented as functional building blocks to emulate diverse types of neuroplasticity (e.g., paired-pulse facilitation/depression (PPF/PPD), spike rate-dependent plasticity (SRDP), and spike time-dependent plasticity (STDP)). ${ }^{[438-443]}$ However, several limitations and drawbacks still remain, for example, CMOS circuits for synaptic simulation require large integration area and power consumption while two-terminal devices still suffer from nonlinearity, large operation voltage as well as the limited conductance modulation range. Meanwhile, signal transmission and learning is hard to process concurrently. 
Three-terminal transistors such as floating-gate memory transistors, ionic/electronic hybrid transistors ${ }^{[444]}$ and organic transistors (CNT-based transistors) ${ }^{[445]}$ are capable of achieving simultaneous signal transmission and learning operations. These two perpendicular channel-based devices can exhibit linear conductance tuning more easily by modulating the gate bias and hence the charge carriers within the semiconducting channel based on charge transport and trapping mechanism. Specifically, for ambipolar floating-gate transistors, the effective injected charge carriers including holes and electrons can induce larger conductance change margin and thereby wider controllable synaptic plasticity compared to unipolar transistors. Besides, injected carriers with opposite polarity are able to neutralize previously captured charges and hence reduce operation bias and energy dissipation. Moreover, ambipolar transistors which possess achievable separated p- and n-channels have the ability to be reconfigured between excitatory and inhibitory modes and hence realize the opposite responses under identical pulse spike, which finally can be utilized to implement dynamic tunable synaptic plasticity and emulate various body perceptions under diverse ambient atmosphere. ${ }^{[446-448]}$ However, ambipolar transistors with these advantages are rarely reported for neuromorphic computing.

In ambipolar synaptic transistors, external action potential (consecutive pulse trains) is exerted onto the vertical gate channel (similar to the stimuli applied onto presynaptic neuron) and postsynaptic current (PSC) is extracted from lateral semiconducting channel between source and drain electrodes (analogous to the potentiation/depression effects of postsynaptic neuron). ${ }^{[447,449]}$ Recently, Tian et al. exploited ambipolar transistors based on the crystal anisotropy of black phosphorus semiconductors to simulate heterogeneous functionality of chemical synapses (Figure 25a). ${ }^{[1]}$ BP channel and its autogenously oxidized compounds $\left(\mathrm{PO}_{x}\right)$ dominated the charge transfer and trapping process and then resulted in synaptic behaviors under different gate pulses. By setting appropriate gate spike parameters, long-term potentiation/depression (LTP/LTD) as well as STDP were realized along various orientations of BP crystals (Figure 25b). In addition, the authors also demonstrated electronic axon-multisynapses network containing four synapses by depositing eight source/drain electrodes (separated by $45^{\circ}$ ) onto anisotropic BP channel (Figure 25c). These synapses exhibited diverse synaptic weights due to different electrical properties under different crystalline orientations of BP film, signifying the heterogeneity of connection strengths in nervous system (Figure 25d). BP-based synaptic transistors possess the potential for achieving complicated biological heterogeneity in neuromorphic computing.

Very recently, Ren et al. fabricated flexible organic ambipolar trapping transistors composed of bendable polyethylene terepthalate (PET) substrate, $\mathrm{Ag}$ gate electrode, $\mathrm{Al}_{2} \mathrm{O}_{3}$ blocking layer, $\mathrm{C}_{60}$ / PMMA hybrid layer $\left(\mathrm{C}_{60}\right.$ functions as floating gate while PMMA acts as tunneling dielectric), pentacene semiconductor as well as Au source-drain electrodes via a simple solution-processed strategy and developed their promising application for simulating chemical synapses (Figure 25e)..$^{[40]}$ Controllable morphologies and charge trapping polarities could be easily achieved by tuning the blending ratios between $\mathrm{C}_{60}$ and PMMA and stable electrical performance with excellent retention and endurance capability was observed at a $0.15 \mathrm{wt} \%$ doping concentration of $\mathrm{C}_{60}$, even after more than 500 repetitive bending events. By comparing dynamic charge transport in transistors and neurotransmitter transfer within synapses, diverse functionalities (excitatory postsynaptic current (EPSC), PPF/PPD, LTP/STP, and multiple learning events) of chemical synapses were mimicked, laying the foundation for subsequent artificial synaptic emulation utilizing organic electronics (Figure 25f).

With the extensive research and many advantages of ambipolar transistors, more and more neuromorphic engineering on the basis of them will emerge soon.

\subsection{Ambipolar Transistor for Logic Electronics}

CMOS inverters are basic units in complementary circuits and composed of $\mathrm{p}$ - and n-type transistors with symmetric threshold voltages as well as analogous response speed. ${ }^{[451-454]}$ In the course of work, the two pairs of gate and drain electrodes of $\mathrm{p}$ - and n-type transistors are linked together and function as input and output terminals. N-type transistor works as drive transistor with its source electrode grounded while p-type transistor acts as load transistor in which electrical power is supplied to its source. Under constant power supply to source, either $\mathrm{p}$ - or n-type transistor can be turned on depending on the input bias and the output signal is contrary to the input voltage; apparently, NOT logic is implemented in CMOS inverters. Besides, minimized current values (equal to leakage current) are achieved inside inverter devices and only exist during short switching operation, which finally gives rise to reduced energy dissipation of integrated circuits in comparison with other single transistor-based inverters.

One traditional approach to manufacture complementary inverters is to utilize two various semiconducting materials for $\mathrm{p}$ - and n-type transistors. However, complicated fabrication procedures such as deposition, pattering and optimization (modulate interfaces between layers) of the semiconductors and low integration density remain a big challenge. Thus, another method employing ambipolar transistors (single semiconductor) for fabricating complementary inverters is more promising. One can operate them in either p- or n-type unipolar mode and hence the processing complexity and cost can be significantly decreased.

In 2003, Meijer et al. first put forward inverter devices on the basis of ambipolar transistors exploiting polymeric blending semiconducting materials ([6,6]-phenyl- $\mathrm{C}_{61}$-butyric acid methyl ester and poly[2-methoxy-5-(3',7'-dimethyloctyloxy)]-pphenylene vinylene $\left.\left(\mathrm{OC}_{1} \mathrm{C}_{10}-\mathrm{PPV}\right)\right) \cdot{ }^{[21]}$ The inverters exhibited gain of 10 due to the relatively small hole and electron mobilities. After that, CMOS inverters on the basis of ambipolar transistors have been widely studied. Zhou et al. demonstrated inverters based on ambipolar transistors which utilized solution-processable layer-by-layer PDPP-TBT/GO hybrids as semiconductor (Figure 26a). ${ }^{[45]}$ The inverters displayed high signal gain as well as sharp switching performance. Nonetheless, how to realize balanced high charge mobilities and low off-currents under unipolar mode is still challenging.

To fabricate high-performance CMOS inverters on the basis of ambipolar transistors, the following electrical properties are 

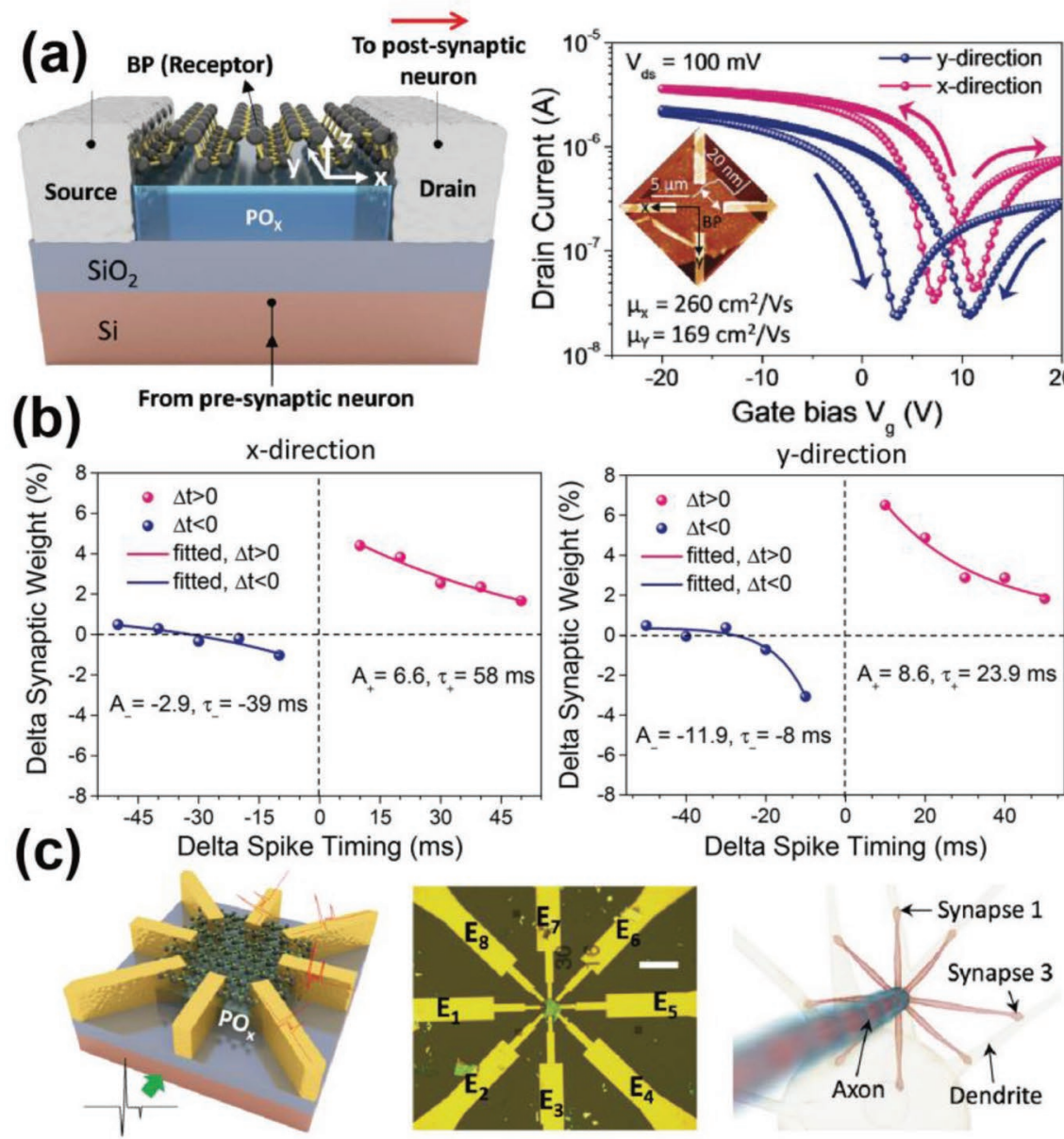

Delta Spike Timing (ms)
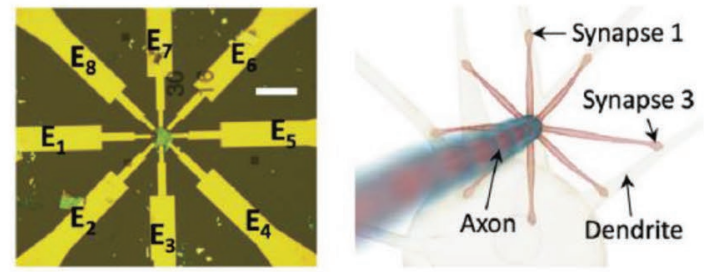

(d)
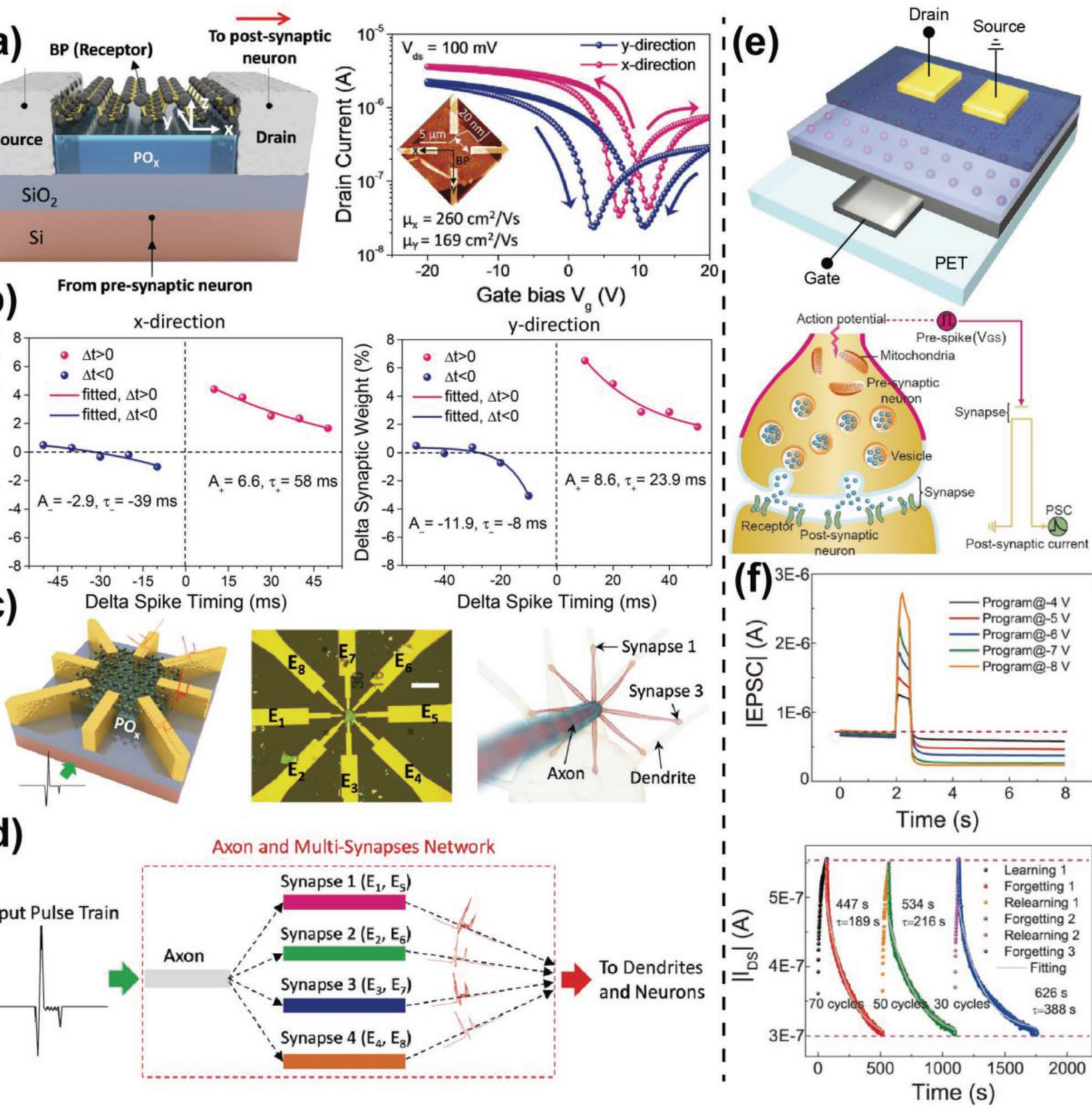

Figure 25. a) Schematic configuration of the synaptic transistors based on BP and representative transfer curves indicating hysteresis along the perpendicular $x$-direction and $y$-direction of BP (charge mobility in $x$-direction is much larger than that in the $y$-direction; the inset shows the AFM graph of the BP-based transistors which were built along two orthogonal directions). b) Anisotropic STDP properties implemented in the $x$ - (left) and $y$-directions (right) of the synaptic transistors, respectively. c) Schematic diagram (left) and optical image (middle) of the synaptic network based on BP as well as illustration of a biosynaptic network (right). d) Measurement schematic of the synaptic transistors. Reproduced with permission. ${ }^{[17]}$ Copyright 2016, Wiley-VCH. e) Device structure of bottom-gate top-contact flexible FET and analogy of chemical synapse and three-terminal flexible synaptic FET. f) EPSC measured under a variety of negative gate pulses with diverse potentiating biases; successful implementation of biological repetitive learning, forgetting and relearning functions through the conductance modulation of the flexible synaptic FET. Reproduced with permission. ${ }^{[450]}$ Copyright 2018 , Wiley-VCH.

required: i) efficient charge carrier injection and well-balanced large mobilities of holes and electrons. Unbalanced charge injection and carrier mobilities can make the switching bias of ambipolar transistors away from their desired value of half of the drain voltage $\left(0.5 V_{\mathrm{DD}}\right)$ and result in unnormal Z-shaped electrical properties of CMOS inverters with reduced noise immunity, lowered DC gain and increased static energy dissipation; ${ }^{[10,34]}$ ii) symmetric electron and hole turn-on voltages. Balanced threshold bias under individual p- and n-channel can induce relatively small operation voltage and hence low power consumption; ${ }^{[456,457]}$ iii) relatively small off current and hence large on/off ratio under unipolar mode. Due to the 
(a)

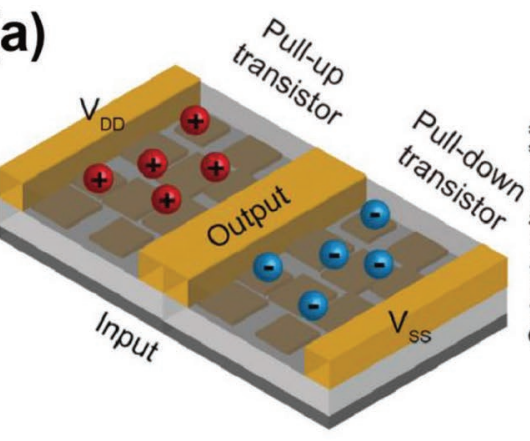

(b) $\square \square^{\text {Inkjet-printing }}$

n-dopant $\odot$ p-dopant
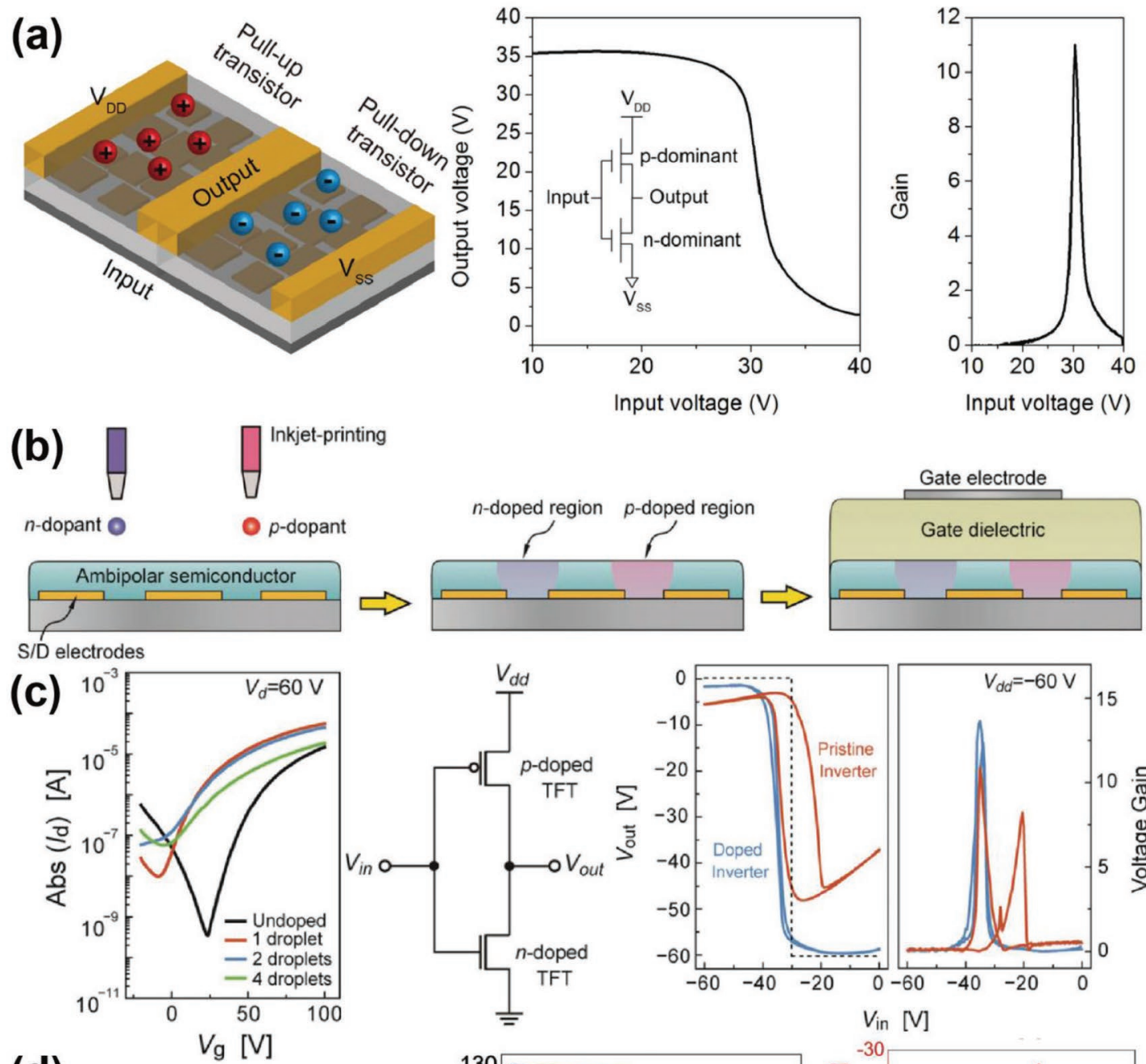

(d)
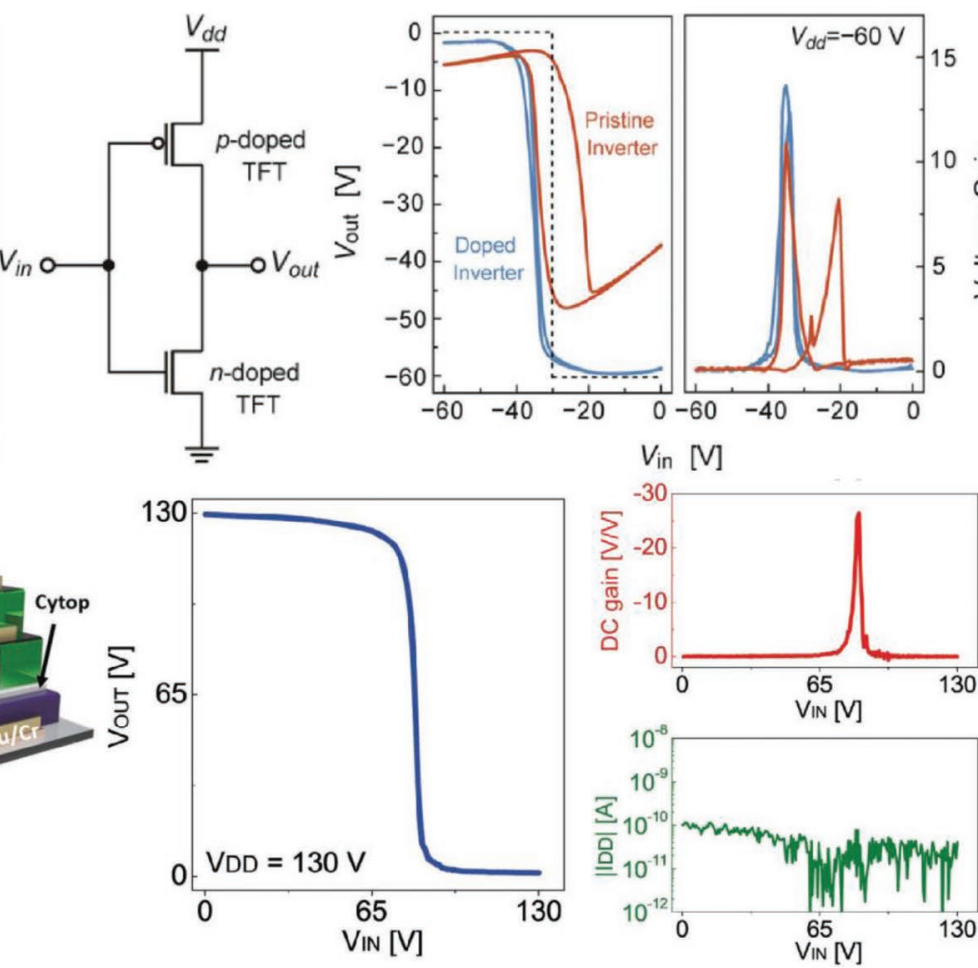

Figure 26. a) Schematic diagram (left), voltage transfer characteristic (middle) and signal gain (right) of CMOS inverters on the basis of PDPP-TBT/ GO hybrids. Reproduced with permission. ${ }^{[455]}$ Copyright 2015, Nature Publishing Group. b) Schematic illustration of manufacturing flow of the CMOS inverters via inkjet-printing approach. c) Representative transfer curves of OFETs on the basis of CsF doped PCBM under various number of droplets; inverter structure based on $\mathrm{p}$ - and n-doped TFTs; voltage transfer curves (left) as well as matching signal gains (right) of different types of inverters (orange solid line, blue solid line and black dashed line represent CMOS inverters with two same FETs based on PCBM and two various transistors on the basis of $\mathrm{p}$ - and $\mathrm{n}$-doped PCBM and ideal electrical characteristic curves of inverters with constant $V_{D D}$ of $-60 \mathrm{~V}$ ). Reproduced with permission. ${ }^{[461]}$ Copyright 2014, Wiley-VCH. d) Schematic of top-split-gate ambipolar OTFT; voltage transfer curve (left), DC gain (top) as well as IDD (bottom) of CMOS inverters on the basis of top-split-gate ambipolar TFTs. Reproduced with permission. ${ }^{[464]}$ Copyright 2018, Wiley-VCH.

inherent ambipolar characteristics of semiconducting materials (electrical current induced by holes augments before the current generated by electrons all passes away), the vast majority of ambipolar transistors are incapable of being completely switched off (without well-defined off-state), which finally gives rise to superabundant leakage and energy consumption, 
reduced gain values as well as decreased noise immunity. ${ }^{[456,458]}$ Therefore, ambipolar transistors with complete voltage switching and thereby large on/off ratio are crucial and urgently required for high-performance CMOS inverters.

In the past few years, to design inverters with low energy dissipation, high gain and noise immunity, researchers have proposed a series of strategies to selectively transport unipolar charges. For example, modification of blocking dielectric and carrier injection electrode contacts via importing or excluding charge traps between the interface of semiconducting and blocking layers, intercalating carrier injection layers as well as utilizing SAMs (adjust the Fermi level of the contact electrodes) both can induce desired transporting polarity. ${ }^{[459,460]}$ Besides, chemical doping is also valid for modulating charge injection, charge mobilities and hence polarities of transistors. Khim et al. demonstrated controllable unipolarization of ambipolar transistors based on PCBM via blending or inkjet printing of tetrafluoro-tetracyanoquinodimethane (p-type F4-TCNQ) or cesium fluoride (n-type $\mathrm{CsF}$ ) dopants onto the semiconducting layer (Figure 26b). ${ }^{[461]}$ It was found that the excess charges (electrons or holes lying with the types of dopants) within semiconducting channel induced by introduced dopants could occupy the deep charge traps. Therefore, the shift of Fermi level can be induced for respective reduced energy barrier to achieve simpler carrier injection. Besides, the unipolar transistors after chemical doping displayed better stability in contrast to original ambipolar ones in identical gate voltage stress and air atmosphere probably on account of the movement of Fermi level toward HOMO/LUMO levels. As a consequence of accurate controllable mobilities and threshold voltages of charge carriers, the inkjet printing inverters presented DC gain of $\approx 14$ and relatively large noise margin (67\% of $1 / 2 V_{\mathrm{DD}}$ ) (Figure $26 \mathrm{c}$ ). However, these methods inevitably increase several fabrication steps and the processing complication.

Another viable means to tune ambipolar transistors into unipolar devices is using multiple gates (e.g., split-gate and tri-gate structures) to control the charge transport electrostatically and directly. ${ }^{[457,462]}$ This method can regulate the polarity of transistors after manufacture and is reversible after withdrawing the gate bias. But the interval between adjacent gates can reduce the drain current significantly and so the gaps should be elaboratively designed (as small as possible). Torricelli et al. fabricated tri-gate transistors on the basis of spin-coating DPP polymeric semiconducting layer, which showed high gain (80) and noise margin ( $6 \mathrm{~V})$, large on/off current ratio $\left(\geq 10^{5}\right)$ as well as low static power $(30 \mathrm{pW})$ when operated at unipolar regime. ${ }^{[463]}$ It was also found that the noise margin augmented by $2 \mathrm{~V}$ when eliminating the spaces between neighboring gates. Utilizing attached metal films for gate electrodes can exclude these gaps but unavoidably improve fabrication cost. In addition, split-gate transistors employing inorganic oxide blocking dielectrics suffer from apparent gate bias stress owing to the existed hydroxyl traps of the oxides. Very recently, Yoo et al. developed top-gate bottom-contact split organic transistors incorporating Parylene-C blocking layer and Cytop hydrophobic layer and demonstrated hysteresis-free electrical characteristics (Figure 26d). ${ }^{[464]}$ Besides, these devices displayed increased gain (about $\left.26 \mathrm{~V} \mathrm{~V}^{-1}\right)$, larger hole $\left(0.46 \mathrm{~cm}^{2} \mathrm{~V}^{-1} \mathrm{~s}^{-1}\right)$ and electron $\left(0.1 \mathrm{~cm}^{2} \mathrm{~V}^{-1} \mathrm{~s}^{-1}\right)$ mobilities due to declined trapped carriers at the interface and improved output swing (99\%) in the complementary inverter. However, the difficulty to obtain balanced carrier mobilities is still a flaw of multigate based inverters.

Except for the above mentioned complementary inverters utilizing the identical semiconducting material for $\mathrm{p}$ - and n-type performance, Huang et al. reported novel in-plane perpendicular aligned ambipolar semiconducting layer ("shishkebab" structure) consisting of electron-deficient P3HT and electron-rich $\quad N, N^{\prime}$-di-n-octyl-3,4,9,10-perylenetetracarboxylic diimide (PDI) via a facile capillary-assisted drop-casting strategy (Figure 27a). ${ }^{[465]}$ Mutually orthogonal P3HT fibers and PDI nanowires could induce separated and independent hole and electron transport along perpendicular directions. Besides, the electrical characteristics of $\mathrm{p}$ - and n-type semiconducting materials such as mobility and threshold voltage could be simply adjusted through modulating the blending concentrations of each material. Furthermore, because of detached respective pand n-channels and easily tunable well-balanced electron/hole mobilities, CMOS inverters (Figure 27b) based on this ambipolar transistor displayed large noise margin $\left(80 \%\right.$ of $\left.1 / 2 V_{\mathrm{DD}}\right)$ (Figure 27c) meanwhile fabricated "NAND" and "NOR" gates presented small steady-state current (in the order of nA) and low static energy dissipation (Figure 27d). Compared to previously stated ambipolar transistors which suffer from balanced charge mobilities and low off-currents, this simple and effective method with separated transport channels provides a new prospective for manufacturing CMOS inverters on the basis of orthogonal ambipolar semiconductors.

In addition to aforementioned inverters based on organic materials, 2D materials have also been utilized to build inverters on account of their outstanding optoelectronic characteristics (especially large on/off ratio) and the rapid development of 2D ambipolar transistors. ${ }^{[466,467]}$ In the last few years, several strategies such as engineering of work function of metal electrodes, electrostatic and chemical doping and ionic liquid gating technique have been exploited to realize efficient ambipolar charge transport in 2D transistors and hence high-performance logic inverters. ${ }^{[286,287,467,468]}$ Next we review the recent progress regarding the utilization of ambipolar 2D materials for manufacturing CMOS inverters (homoinverters) and many other circuits based on various 2D material systems.

With respect to molybdenum dichalcogenides, $\mathrm{MoS}_{2}$ is best known and hence attracts widespread concern. ${ }^{[469-472]}$ Singlayer $\mathrm{MoS}_{2}$ possesses a direct energy gap of $1.8 \mathrm{eV}$ while bulk (2H) $\mathrm{MoS}_{2}$ with hexagonal architecture displays relatively small energy gap of $\approx 1.2 \mathrm{eV} \cdot{ }^{[473,474]} \mathrm{MoS}_{2}$ always exhibits n-type carrier conduction behavior, which remains unchanged by modulating the gate bias or using metal electrodes with large work function due to its wide energy gap and intense Fermi level pinning. ${ }^{[473-476]} \mathrm{MoO}_{x},{ }^{[477]}$ ion gel gating, ${ }^{[302]}$ chemical doping, ${ }^{[478]}$ as well as surface plasma ${ }^{[479]}$ both can be utilized for achieving hole transport in $\mathrm{MoS}_{2}$ transistors. In 2011, Radisavljevic et al. reported the first inverter on the basis of single-layer $\mathrm{MoS}_{2}$, which showed large voltage gain of more than $4 .{ }^{[480]}$ Later, in 2012, Wang et al. demonstrated several integrated circuits (e.g., NAND gate and five-stage ring oscillator) on the basis of bilayer $\mathrm{MoS}_{2}$ FETs and the fabricated inverter presented high gain value of $\approx 5 .{ }^{[481]}$ Recently, Wachter et al. fabricated highperformance CMOS inverters on the basis of planar $\mathrm{MoS}_{2}$ via 
(a)
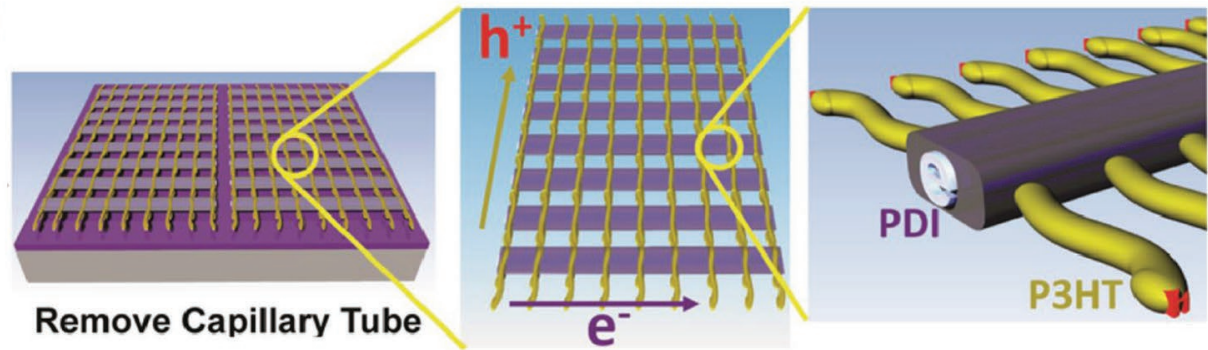

(b)

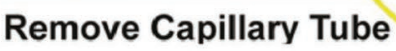

$\mathrm{e}^{-}$
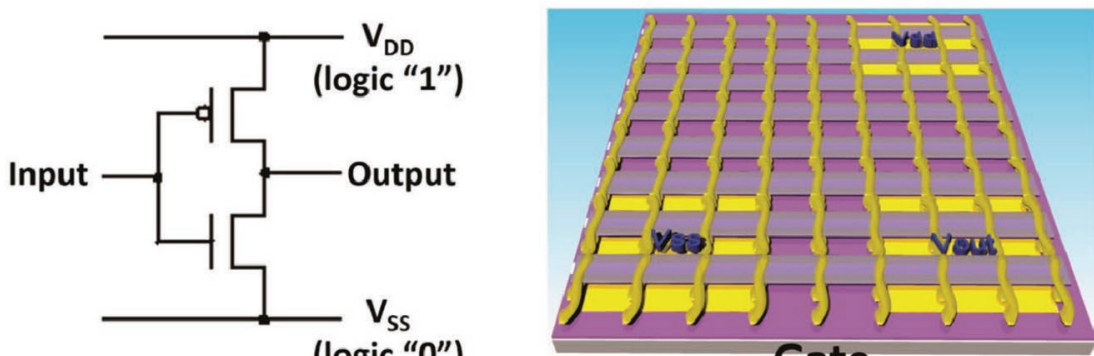

(c)

(logic "0")

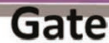

(d)
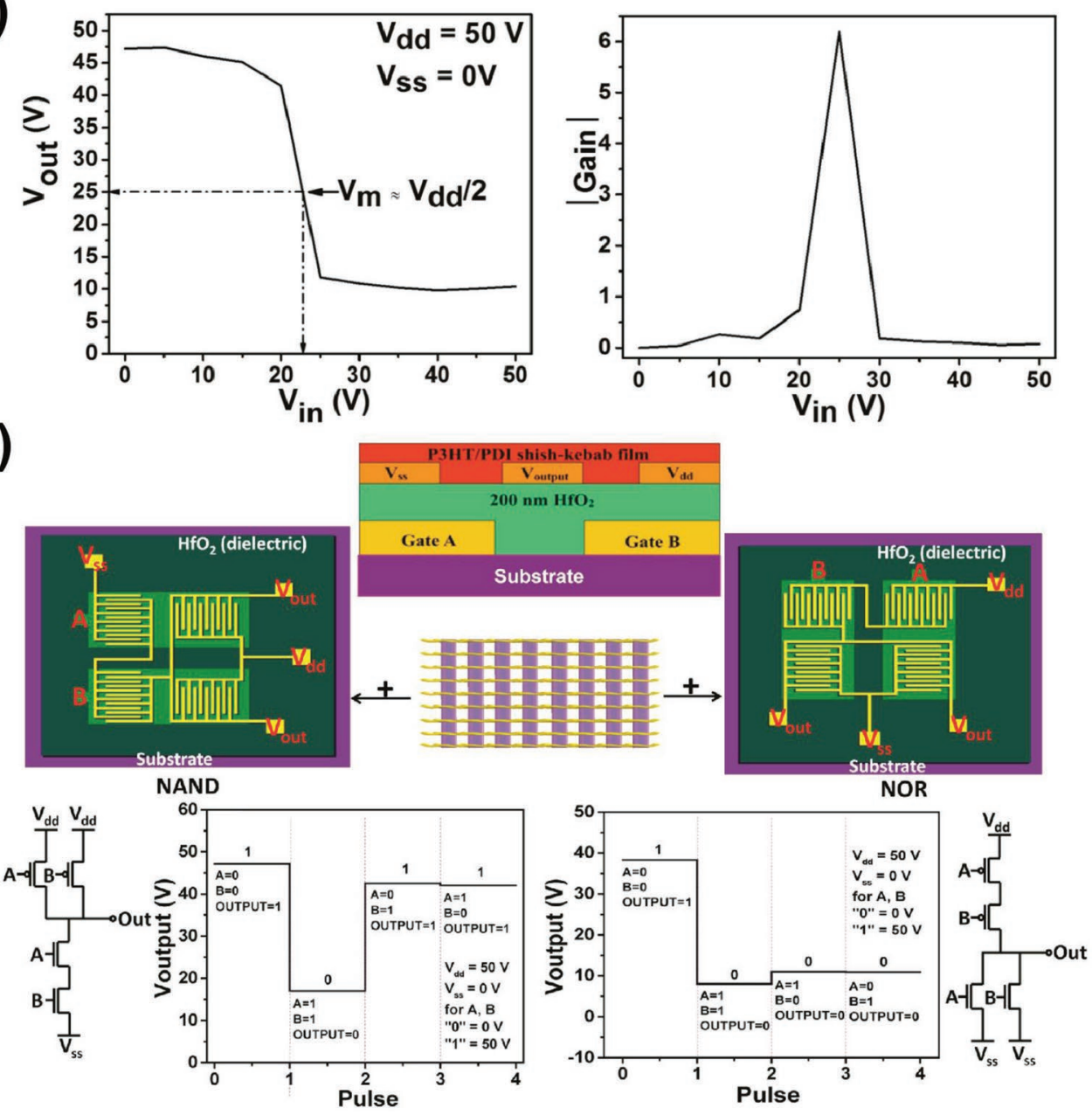

Figure 27. a) Schematic configuration of aligned P3HT (hole transport)/PDI (electron transport) "shish-kebab" structure. b) Schematic of CMOS inverters on the basis of "shish-kebab" perpendicular ambipolar semiconducting layer. c) Voltage transfer characteristic (left) and gain (right) of the aforementioned inverter. d) Schematic illustration of the cross-sectional image of circuit structure, aligned P3HT/PDI orthogonal films as well as the circuit layout of "NOR" and "NAND" circuits; voltage transfer characteristics of the above mentioned two circuits ("NAND": left; "NOR": right). Reproduced with permission. ${ }^{[465]}$ Copyright 2016, American Chemical Society. 
CVD. ${ }^{[482]}$ The $\mathrm{MoS}_{2}$ FET presented extremely large on/off ratio (about $10^{8}$ ) and homogeneous performance within a small region of $\approx 50 \mathrm{~mm}^{2}$ in the whole wafer. The inverter exhibited extremely high voltage gain of 60 at $V_{\mathrm{DD}}=5 \mathrm{~V}$. Apart from $\mathrm{MoS}_{2}, \mathrm{MoTe}_{2}$ has also been extensively investigated to achieve ambipolar carrier conduction as well as high-performance inverter devices. ${ }^{[312,316,483-486]}$ Lim et al. exploited atomic-layerdeposition-generated $\mathrm{H}$-doping to achieve electron-dominated $\alpha$-MoTe 2 flake with electron mobility of $18 \mathrm{~cm}^{2} \mathrm{~V}^{-1} \mathrm{~s}^{-1}$, which finally gave rise to stable CMOS inverters with large gain value (29) and small static power dissipation on the order of nanowatts. ${ }^{[487]}$ Very recently, by combining $\mathrm{MoTe}_{2}$ with $\mathrm{BN}$ and forming a heterostructure between them, Liu et al. proposed a photodoping method to accurately adjust the electron conduction characteristics (concentration and mobility of electrons) of $\mathrm{MoTe}_{2}$ FET via tuning the amplitude of the applied photodoping gate. ${ }^{[488]}$ It was found that the induced photodoping effect was repeatable and nonvolatile and could be maintained for more than 14 days without ambient stimulation. Photoresist-free homoinverters of $\mathrm{MoTe}_{2}$ were also fabricated by regulating the photodoping area on semiconducting channel and the built inverters basically had no interfacial residues (tidy interface), which finally resulted in tremendous DC voltage gain as large as 98 at $V_{\mathrm{DD}}=3 \mathrm{~V}$ (Figure $28 \mathrm{a}-\mathrm{c}$ ).

In regard to tungsten dichalcogenides, $\mathrm{WSe}_{2}$ is the most proverbially studied material for building CMOS inverters by reasons of its inherent ambipolar characteristic, wide energy gap of $1-2 \mathrm{eV}$ and ultrathin thickness of $<1 \mathrm{~nm} .^{[311,489]}$ Besides, FETs based on $\mathrm{WSe}_{2}$ display large charge carrier mobilities of more than $100 \mathrm{~cm}^{2} \mathrm{~V}^{-1} \mathrm{~s}^{-1}$, on/off ratio of exceeding $10^{6}$ and relatively small subthreshold swing of $60 \mathrm{mV}$ decade $^{-1}$ due to the ultrathin semiconducting channel and hence better electrostatic modulation, ${ }^{[286,293,490]}$ which provides lots of advantages to fabricate low-power logic inverters with extremely low off-state voltage and large gain value. ${ }^{[491-494]}$ Recently, $\mathrm{Pu}$ et al. demonstrated high-performance CMOS inverters on the
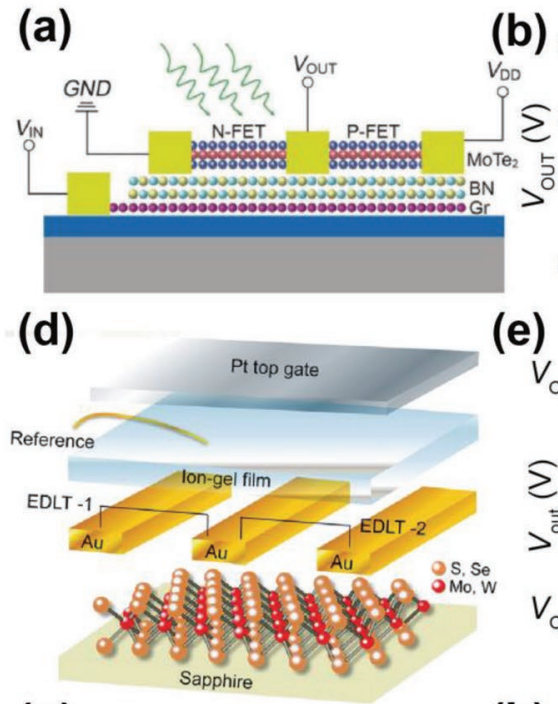

(g)

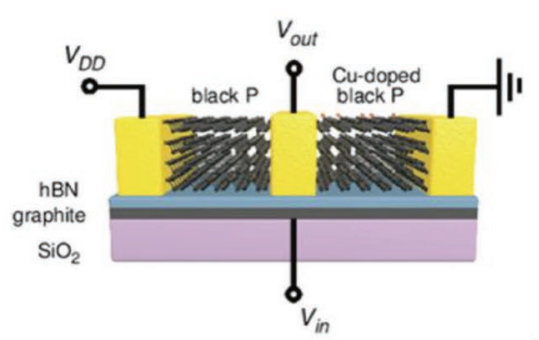

(e)
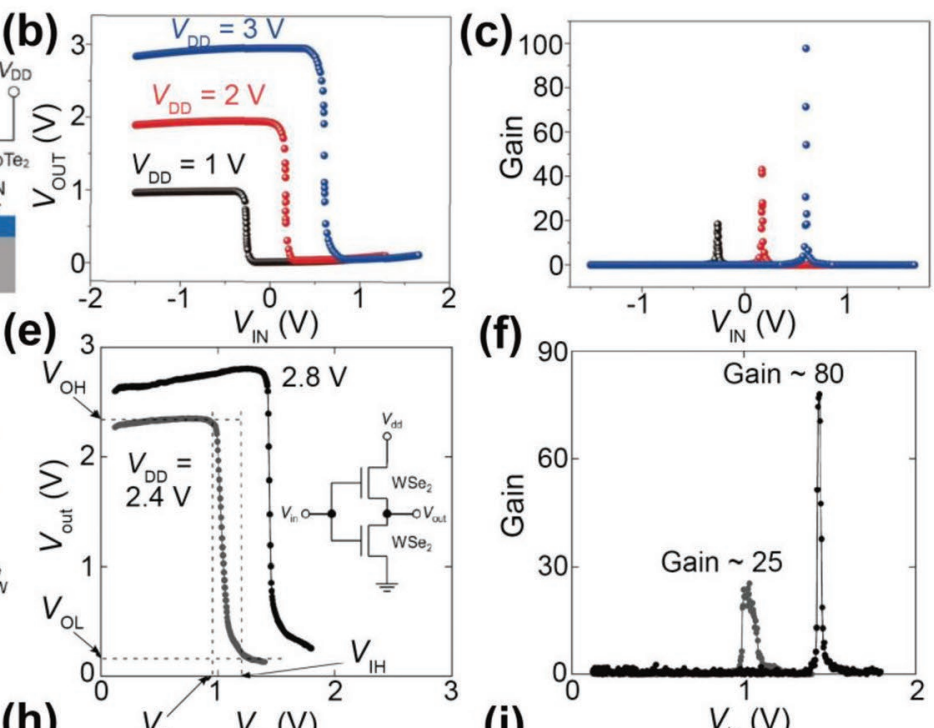

(f)
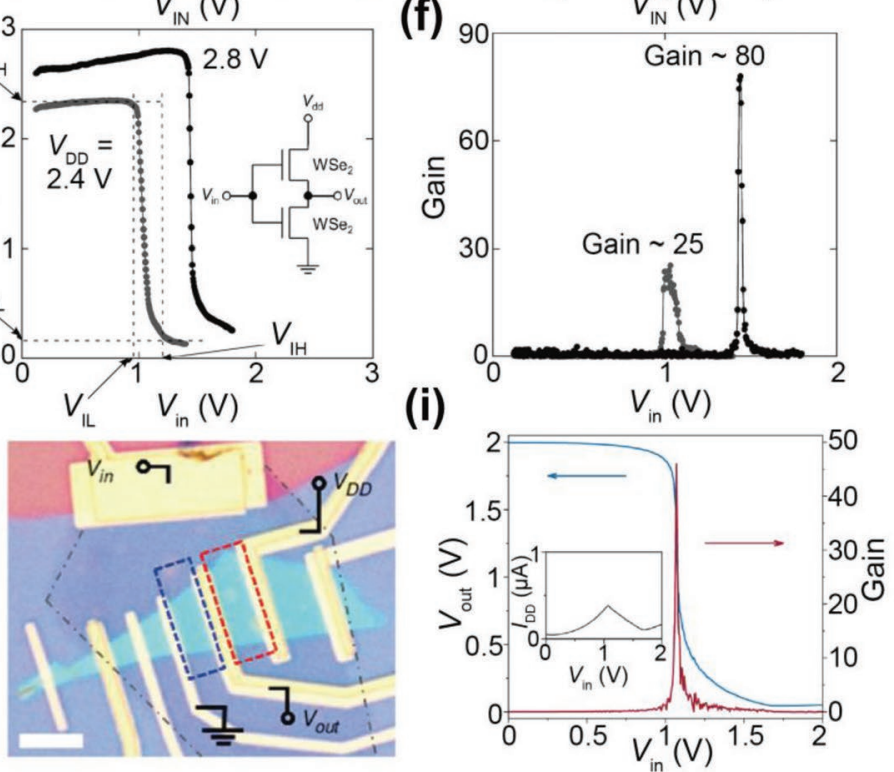

Figure 28. a) Schematic diagram of the logic inverter on the basis of $\mathrm{MoTe}_{2}$ realized via modulating the photodoping region. $\mathrm{Graphene}, \mathrm{BN}$, and $\mathrm{MoTe} \mathrm{e}_{2}$ function as gate electrode, blocking dielectric and semiconducting channel, respectively. The left semiconducting layer is irradiated to induce n-type $\mathrm{MoTe}_{2}$ FET whereas the right layer is not exposed to laser beam and generates a p-type MoTe 2 FET. b) The typical output curve of the aforementioned inverter under various $V_{D D}$ values $(1,2$, and $3 \mathrm{~V})$. c) The obtained voltage gain values of the MoTe $\mathrm{M}_{2}$ inverter under different input biases. It is found that the maximum gain value increases from 18, 42, to 98 when $V_{D D}$ augments from 1, 2 to 3 V. Reproduced with permission. ${ }^{[488]}$ Copyright 2018, Wiley$\mathrm{VCH}$. d) Schematic illustration of the electric double layer transistor based on monolayer $\mathrm{WS}_{2}$ and ion gel dielectric. e) Voltage transfer properties of the aforementioned inverter under diverse $V_{D D}$ values $\left(2.4\right.$ and $2.8 \mathrm{~V}$ ). The inset displays the circuit schematic of the WSe $\mathrm{W}_{2}$ inverter which contains two series-wound electric double layer transistors on the basis of $\mathrm{WSe}_{2} . \mathrm{f}$ ) The obtained gain values of the inverter at various $V_{\text {in }}$ values. The highest voltage gain value is 80 at $V_{D D}=2.8$ V. Reproduced with permission. ${ }^{[495]}$ Copyright 2016, Wiley-VCH. g) Schematic diagram of the fabricated BP CMOS inverter which consists of a Cu-doped n-type transistor and an unprocessed p-type transistor. h) Optical image of the aforementioned BP inverter (scale bar: $5 \mu \mathrm{m}$ ). i) Output voltages, gain values and device currents at different input voltages and constant $V_{D D}$ of 2 V. Reproduced with permission. [502] Copyright 2016, American Chemical Society. 
basis of CVD-grown $\mathrm{WSe}_{2}$ monolayers and ion gel dielectric (Figure 28d). ${ }^{[495]}$ The inverters presented maximum voltage gain as large as 80 at $V_{\mathrm{DD}}=2.8 \mathrm{~V}$ because of the large on/off ratio and charge mobilities of $\mathrm{WSe}_{2}$ transistors (Figure 28e,f).

Black phosphorus is another commonly used 2D crystal for efficient CMOS inverters as a result of its superior electrical properties such as extremely large hole mobility of $1000 \mathrm{~cm}^{2} \mathrm{~V}^{-1} \mathrm{~s}^{-1}$ and good on/off current ratio of $10^{5} \cdot{ }^{[332,496-501]}$ Recently, Koenig et al. investigated the influence of $\mathrm{Cu}$ adatoms on BP and found that the introduced $\mathrm{Cu}$ adatoms could make BP exhibit n-type carrier conduction (electron mobility: $2140 \mathrm{~cm}^{2} \mathrm{~V}^{-1} \mathrm{~s}^{-1}$ under relatively low temperature) without lowering original charge transport characteristics. ${ }^{[502]}$ By exploiting BN, graphite and unitary BP crystal as blocking dielectric, gate electrode and semiconducting channel, their fabricated logic inverters showed remarkably large gain value of 46 thanks to the high mobilities of BP FETs (Figure 28g-i). With the rapid development and concentrated research of 2D materials, 2D CMOS inverters with higher voltage gain, larger noise immunity and lower power consumption are expected.

\subsection{Ambipolar Light-Emitting Transistors}

Ambipolar light-emitting transistors (LETs) represent multifunctional architectures which integrate switching characteristic (property of transistors) and light emission capacity (nature of LEDs) into a unitary device. ${ }^{[117,503-509]}$ In ambipolar LETs, electrons and holes can be injected from source-drain electrodes to gate-tunable semiconducting channel and then recombine with each other to emit light with specific wavelength..$^{[510-512]}$ On the grounds of recombination theory, each ambipolar transistor is capable of functioning as LET but their luminous intensity will differ from each other and mainly rest with the electronic properties of the semiconductors (e.g., ampere density and quantum efficiency). LET geometry is superior to conventional LED architecture in many respects such as larger conductivity of semiconducting channel, well-balanced electrons and holes and controllable location of emission zone via modulating gate bias, larger external quantum efficiency (EQE) and significantly reduced loss of excitons, etc. After the first LET based on polycrystalline tetracene semiconducting layer was proposed by Hepp et al. in 2003, ${ }^{[513]}$ ambipolar devices have been extensively investigated.

Researchers have discovered that larger EQE stability of ambipolar LETs based on organic semiconducting materials could be obtained when operated under larger ampere density compared to that of OLEDs. The first bottom-contact top-gate ambipolar LETs utilizing polymers were reported by Zaumseil et al. and the devices exhibited extremely large ampere density ${ }^{[514]}\left(50 \mathrm{~A} \mathrm{~cm}^{-2}\right.$; similar to ambipolar LETs based on rubrene single crystal $\left.{ }^{[515]}\right)$. EQE of the ambipolar LETs remains stable even under large ampere density up to $4 \mathrm{kA} \mathrm{cm}^{-2}$,[15] which outbalances the detrimental roll-off phenomena of OLEDs under small ampere density (about $1 \mathrm{~A} \mathrm{~cm}^{-2}$ ). ${ }^{[516]}$ Later, by decreasing the influence of electron traps, LETs based on 5,5'-bis(biphenylyl)-2,2':5',2"-terthiophene (BP3T) single crystals displayed remarkably large current densities $\left(12.3 \mathrm{kA} \mathrm{cm}^{-2}\right)$, which extended the performance restrictions of ambipolar LETs on the basis of single crystals. ${ }^{[517]}$

Most ambipolar LETs are comprised of single semiconducting active layer which is in charge of both charge transport and light emission functionalities whereas OLEDs usually utilize three separated individual layers (electron transport layer, hole transport layer and emissive layer) to achieve these functions. Thus, it is difficult to contrast the performance of ambipolar LETs and OLEDs directly. Recently, Capelli et al. designed ambipolar LETs on the basis of heterostructured trilayer-based semiconductor and compared them with their counterparts (OLEDs with identical trilayer architecture) (Figure 29a,b). ${ }^{[518]}$ It was found that ambipolar LETs displayed much higher EQE (5\%) than OLEDs (2.2\%) on account of inhibited exciton quenching and minimized loss of photons. In addition, Gwinner et al. reported ambipolar LETs possessing larger EQE of more than $8 \%$ and higher luminance efficiency exceeding $28 \mathrm{~cd} \mathrm{~A}^{-1}$ via tuning light outcoupling of transistors utilizing polymeric semiconducting poly $(9,9$ dioctylfluorenealt-benzothiadiazole) (F8BT) (Figure 29c,d). ${ }^{[519]}$ Furthermore, photonic crystals are also utilized in ambipolar LETs to improve their optoelectronic performance. ${ }^{[504,507]}$ Natali et al. fabricated transparent ambipolar LETs which exploited multilayer high-capacitance photonic crystal $\left(\mathrm{ZrO}_{2}\right)$ $\mathrm{Al}_{2} \mathrm{O}_{3}$ ) as blocking dielectric. ${ }^{[507]}$ By modulating the energy gap of the aforementioned photonic crystal, the emitted power as well as the brightness of the fabricated LETs are an order of magnitude larger than original LETs on the basis of polymeric dielectric. With these merits and gradually tackled technical difficulties (e.g., utilization of phosphorescence matters ${ }^{[520]}$ ), ambipolar LETs are extremely alternative for practical display and lighting applications.

Ambipolar LETs are also exploited extensively for achieving electrically driven organic lasers since the maximum ampere density (1-10 A cm $\mathrm{cm}^{-2}$ ) of OLEDs is too small to electrically pump lasers $\left(>10 \mathrm{kA} \mathrm{cm}^{-2}\right) \cdot{ }^{[521,522]}$ Organic lasers are prospected to possess plentiful advantages in comparison with conventional optically pumped lasers: i) larger laser efficiency due to dramatically reduced laser threshold power for organic semiconducting materials; ii) emitted laser beam with expansive optical wavelength scope; iii) possibility to integrate lightemitting device into several various platforms.

In spite of reduced required laser threshold for organic lasers, the relatively low ampere density induce by inherent small charge mobilities of organic semiconducting materials is difficult for realizing population inversion. Besides, the transistor architectures may lead in other probable loss routes of excitons and thereby augment laser threshold power. Sawabe et al. demonstrated unprecedently large ampere density up to $33 \mathrm{kA} \mathrm{cm}^{-2}$ based on single-crystal ambipolar LETs utilizing BP3T semiconductor. ${ }^{[107,117]}$ However, the lasing threshold was increased $\left(1 \mathrm{kA} \mathrm{cm}^{-2}\right)$ owing to declined exciton quenching. One valid strategy to reduce exciton density and hence lasing threshold is to integrate ambipolar LETs with several optical architectures for instance Fabry-Pérot cavity, ${ }^{[112]}$ distributed feedback (DFB) resonator ${ }^{[523]}$ and microcavity ${ }^{[524]}$.

However, utilizing ambipolar LETs for lasing still exist many challenges and require further investigation. In terms of materials, scientists hunt for novel semiconductors with 


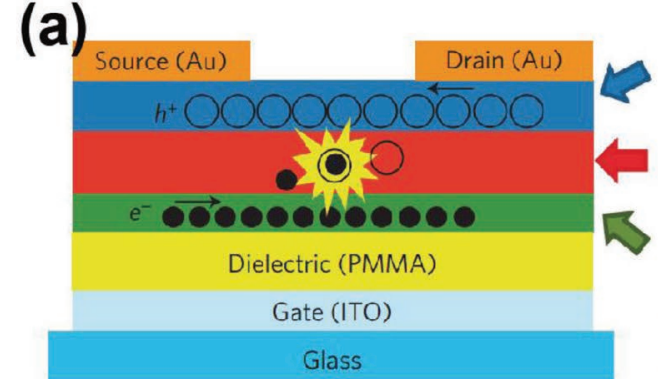

(b)

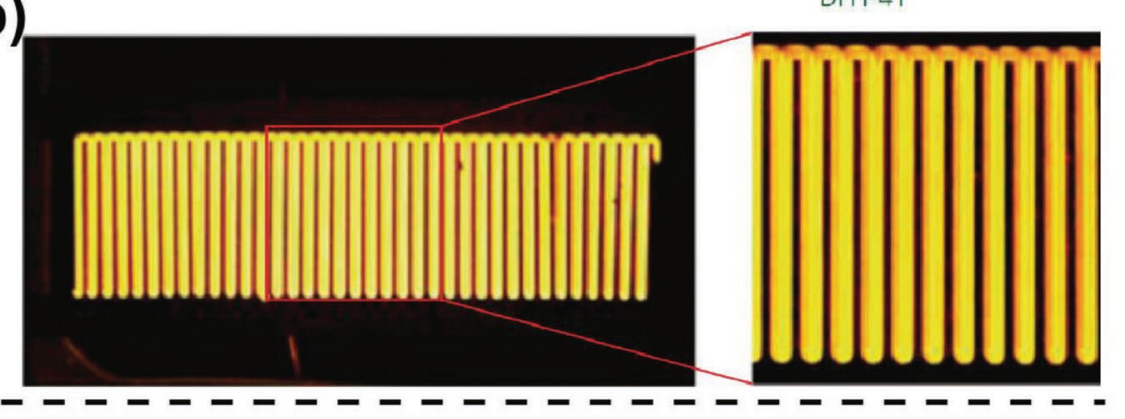

(c)
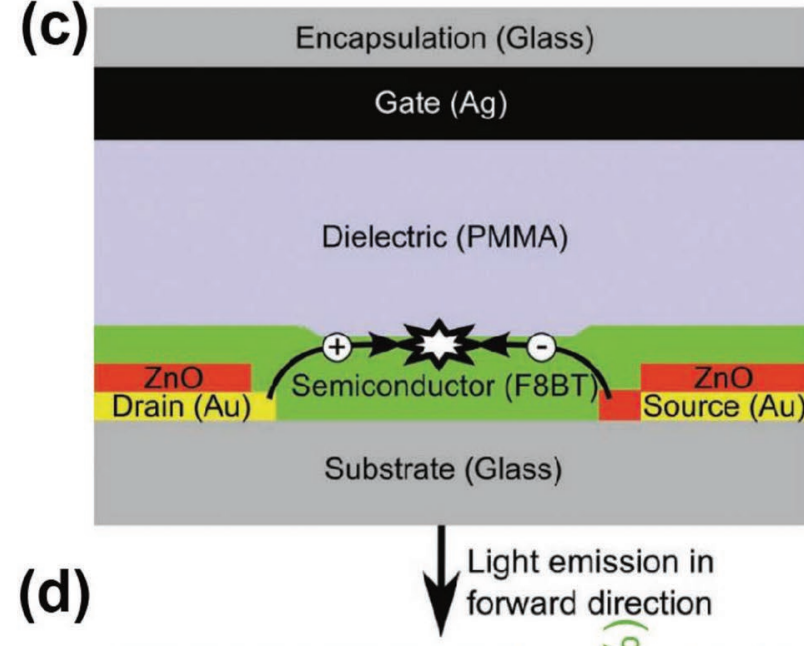
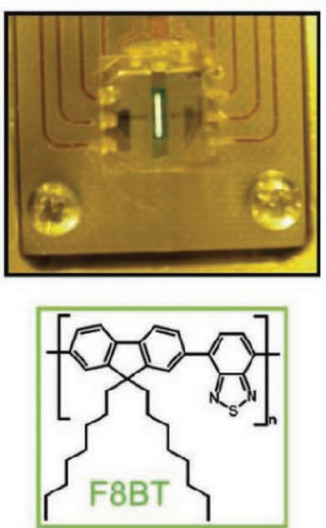

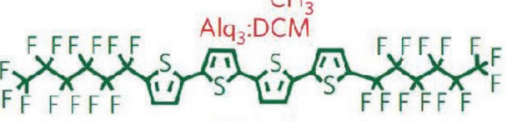

DFH-4T

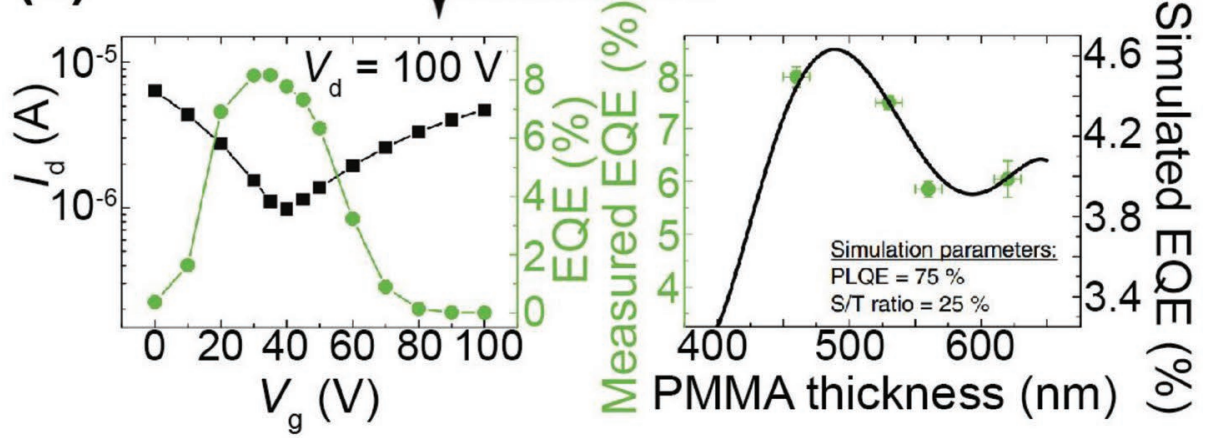

Figure 29. a) Schematic illustration of the ambipolar OLET and molecular structures of the organic materials utilized in the active layers. b) Optical image of the aforementioned trilayer OLET (channel length: $150 \mu \mathrm{m}$; channel width: $20 \mathrm{~cm}$ ). Reproduced with permission. ${ }^{[518]}$ Copyright 2010, Nature Publishing Group. c) Schematic diagram of light-emitting FET based on F8BT (insets describe the mounted sample (top) and the chemical structure of F8BT (bottom)). d) Representative transfer curve and EQE of light-emitting FET (the thickness of PMMA is $460 \mathrm{~nm}$ ); tested and simulated EQEs of light-emitting FETs with various thicknesses of PMMA. Reproduced with permission. ${ }^{[519]}$ Copyright 2012 , Wiley- $\mathrm{VCH}$.

both large luminescence efficiency and good ambipolar charge transport properties ${ }^{[525,526]}$ and novel materials with large singlet excitons density (meanwhile minimized routes to produce triplet excitons) $)^{[527]}$ and exploit molecules eliminating triplet excitons. ${ }^{[528,529]}$ Another perspective is the optimization of transistor architecture and operation, which can improve the 
ampere density and enhance the creation of singlet excitons. Lots of efforts have been made in current crowding, ${ }^{[117]}$ adjustment of recombination size, ${ }^{[530]}$ operation at alternating current mode, ${ }^{[531]}$ influence via magnetic field, ${ }^{[532]}$ etc.

The planar architecture of ambipolar LETs is beneficial for investigating the recombination operation within semiconducting channel through researching the shape and width of the recombination zone. ${ }^{[13,530,533]}$ Since recombination zone locates at the interface between p- and n-type accumulation regions, charge injection efficiency of the ambipolar LETs can be further studied from this zone (e.g., measurement of second harmonic generation emission ${ }^{[534]}$. In addition, ambipolar LETs functioning as probes are implanted to monitor the emission zone transfer process to observe the semiconductor morphology as well as the existed charge traps, signifying their further applications for learning the charge transport characteristics of semiconducting channel. ${ }^{[535,536]}$

Ambipolar LETs based on 2D materials (especially transitionmetal dichalcogenides) have also attracted widespread attention due to their unique optoelectronic characteristics. ${ }^{[537]}$ Monolayer TMDs possess direct energy gap with adjustable lightemitting characteristics and generate light emission depending on closely combined excitons on account of improved Coulomb interactions. ${ }^{[311,538,539]}$ To fabricate high-performance ambipolar 2D LETs based on monolayer TMDs, high- $k$ (high capacitance) blocking dielectrics, for instance $\mathrm{HfO}_{2}$, are always utilized by reasons of their unique advantages such as effective charge density regulation and extensive band filling. ${ }^{[286,293,490,540]}$ In this respect, electric double layer transistor architecture in which electrolytes (e.g., ionic liquid) serve as blocking dielectrics is more commonly used for ambipolar 2D LETs due to its large capacitance $\left(\approx 10 \mu \mathrm{F} \mathrm{cm}{ }^{-2}\right)$, enhanced electric field and thereby increased charge density accumulation within semiconducting channel. ${ }^{[541-543]}$ These superior characteristics result in better ambipolar carrier conduction behaviors (e.g., small operation bias of less than $4 \mathrm{~V}$ and large charge mobility of more than $100 \mathrm{~cm}^{2} \mathrm{~V}^{-1} \mathrm{~s}^{-1}$ ) of TMDs and BP. ${ }^{[302,323,336,492,495,544]}$

The operation mechanism of electric double layer transistor is displayed in Figure $18 \mathrm{c}$ (take $\mathrm{MoS}_{2}$-based electric double layer transistor as an example). ${ }^{[300]}$ When $V_{\mathrm{GS}}$ is far greater than $V_{\mathrm{DS}}$, only a sort of charge is gathered within the semiconducting channel (top image of Figure 18c). For comparison, when $V_{\mathrm{GS}}$ is far less than $V_{\mathrm{DS}}$, the influence of gate bias on the region near the drain electrode is diminished and hence both two types of charge carriers are accumulated within the semiconducting channel (bottom image of Figure 18c). Based on these operating principles, different ambipolar 2D LETs have been fabricated and their electroluminescence spectra have also been investigated. ${ }^{[305,321,545-548]}$ Figure 30a,b exhibits visible electroluminescence picture of electric double layer transistor on the basis of monolayer $\mathrm{WS}_{2}$ and its electroluminescence spectrum. ${ }^{[321]}$ Despite the indistinct electroluminescence image, the photoluminescence spectrum is in good agreement with the electroluminescence spectrum, which demonstrates the direct-gap exciton electroluminescence of $\mathrm{WS}_{2}$. In addition, recently, bulk $\mathrm{ReS}_{2}$ with indirect bandgap property also presents electroluminescence due to the high capacitance (large charge and current density and hence enhanced charge recombination in $\mathrm{ReS}_{2}$ semiconductor) of $\mathrm{ReS}_{2}$-based electric double layer transistor, ${ }^{[544]}$ which signifies that combining electrolyte blocking dielectrics with TMDs is extremely effective to achieve electroluminescence within 2D semiconducting materials.

2D monolayer TMDs also possess special electronic architectures because of the spatial-inversion asymmetry as well as intense spin-orbit interactions. ${ }^{[549-551]}$ Due to the fact that the electronic states of hole and electron valleys in monolayer TMDs possess various chiralities owing to the absent inversion symmetry, interband transitions at $\mathrm{K}^{\prime}$ and $\mathrm{K}$ points are allowed for $\sigma_{-}$and $\sigma_{+}$circularly polarized light, respectively. ${ }^{[550]}$ Monolayer $\mathrm{MoS}_{2}, \mathrm{WSe}_{2}$ as well as several other TMDs both show circularly polarized photoluminescence. ${ }^{[552-556]}$ To achieve spin-valley light-emitting transistors, regulating the number of polarized charges in each valley to interconvert valley polarization to emitted or absorbed photons is crucial. ${ }^{[537]}$ Up to now, two methods are typically utilized for fabricating chiral lightemitting devices based on TMDs. The first approach is utilizing ferromagnetic semiconducting materials or contacts to inject spin-polarized charges and hence generate circularly polarized electroluminescence. ${ }^{[557,558]}$ Another strategy is exploiting strong lateral electric fields to regulate circularly polarized electroluminescence. ${ }^{[546-548,559,560]}$ Zhang et al. put forward an electrical switchable $\mathrm{WSe}_{2}$-based LET on the basis of the valley degree of freedom (Figure 30c). ${ }^{[548]}$ The $\mathrm{p}-\mathrm{i}-\mathrm{n}$ junctions were electrostatically formed within $\mathrm{WSe}_{2}$ semiconductor and then two types of charge carriers flowed to the intrinsic region to recombine and emit photons. It was also found that the electroluminescence possessed a circular polarization which was electrically regulatable via modulating the current direction (Figure 30d). Despite the rapid development of controlling circularly polarized light emission, challenges still remain in how to obtain valley light-emitting devices which are able to work well at room temperature because of the fact that most valley polarizations are measured under relatively low temperature $(<80 \mathrm{~K})$ except several peculiar situations..$^{[546-548,557-559]}$ In addition, more efforts should be paid to reveal the inner mechanisms of polarized electroluminescence generation and charge recombination behaviors dominated by valley degree of freedom.

\section{Conclusions, Challenges, and Prospective}

In summary, we have reviewed recent advances of ambipolar transistors from different aspects comprising fundamental working principles, selected organic, inorganic and organicinorganic hybrid materials, various structures and fabrication methods as well as the diverse applications of ambipolar transistors. In particular, for functional applications, ambipolar transistors which can be operated in $\mathrm{p}$ - or n-type mode are beneficial to fabricate CMOS inverters and hence logic circuits owing to decreased device components and favorable miniaturization and manufacturing process. Intriguingly, ambipolar transistors can be transformed into flash memory via depositing additional floating gate and tunneling layer and thereby exhibit superior memory performance such as large memory window and multilevel storage. Furthermore, by utilizing gate terminal for simulating learning function and source-drain lateral channel for mimicking signal transmission, ambipolar transistors can also be implemented for artificial synaptic 
(a)

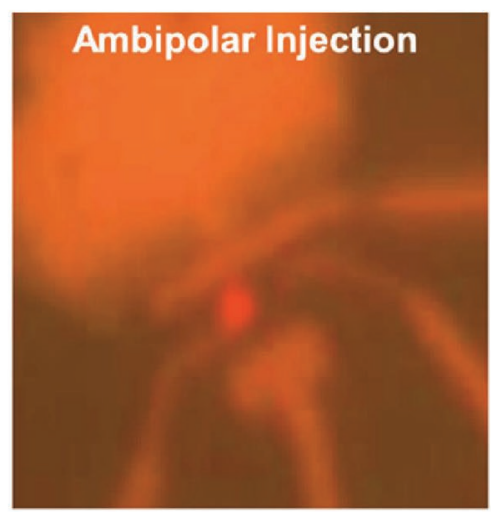

(c)

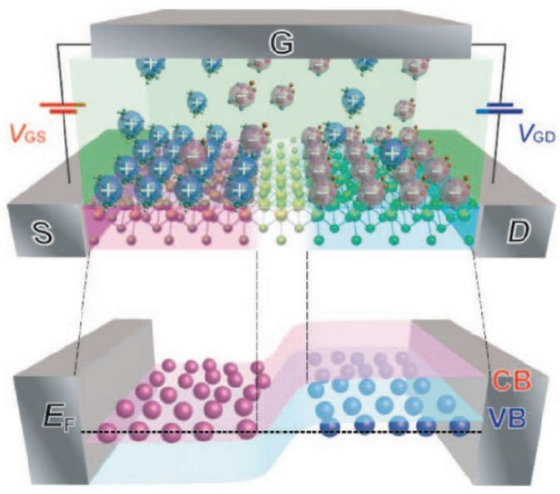

(b)
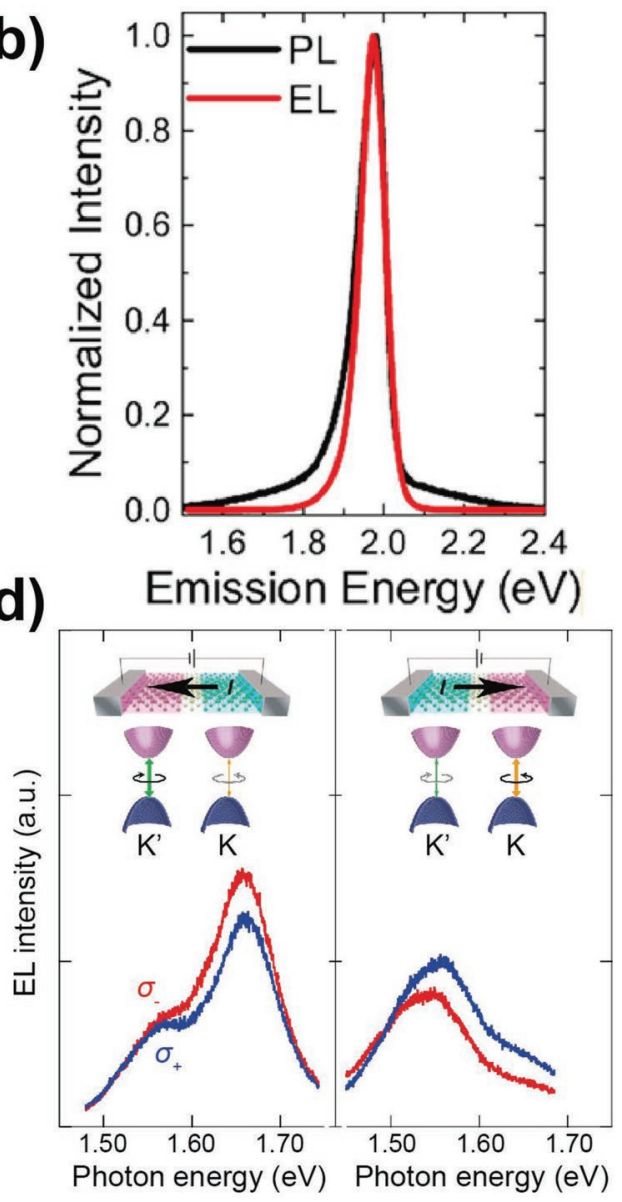

Figure 30. a) Electroluminescence picture obtained from ambipolar electric double layer transistor on the basis of $W_{2} \mathrm{~S}_{2}$ monolayer. b) Observed photoluminescence and electroluminescence spectra of $\mathrm{WS}_{2}$-based electric double layer transistor. Reproduced with permission. ${ }^{[321]}$ Copyright 2014 , American Chemical Society. c) Device configuration (top) and energy band alignment (bottom) of WSe $\mathrm{Se}_{2}$-based electric double layer transistor. Bottom image also shows the generated $\mathrm{p}-\mathrm{i}-\mathrm{n}$ junction at equilibrium state. d) Circularly polarized electroluminescence spectra of WSe $\mathrm{e}_{2}$-based LET at various current directions. The inset presents different contributions of the two valleys for electroluminescence. Reproduced with permission. ${ }^{[548]}$ Copyright 2014, The American Association for the Advancement of Science.

emulation. The rapid progress of ambipolar transistor field has impelled it to become extremely attractive candidate in lighting, display, data storage as well as artificial intelligence, signifying its gradual development, completion and ripeness from academic research to industrial application.

Large and well-balanced electron and hole mobilities, air stability as well as operational reliability of ambipolar transistors have always been pursued as the goals of scientists and thereby the influence of semiconducting materials (HOMO/LUMO, $\pi$-system overlap, molecular packing, etc.), symmetric and asymmetric electrodes, device configurations, interface contacts, etc., on ambipolar performance have been under proverbially studied. Nonetheless, several significant challenges and drawbacks still exist and need overcoming. For organic semiconducting materials: i) the relation between chemical structure and electrical characteristic is still not fully understood and theory-based 2D lamellar packing and mostly reported herringbone packing motif are favorable to obtain organic materials with large hole and electron mobilities; ii) how to make the actual presented ambipolar performance basically the same as the theoretical predicted ambipolar transport on the basis of the inherent characteristics of organic semiconductors remains a tricky subject. The purity of semiconducting materials, interface contacts, film morphology as well as fabrication methods and conditions, etc., both can induce deviations between the actual and theoretical electronic properties, thus, the deviations can be significantly reduced via optimizing these influence factors; iii) although several valid chemical methods are demonstrated triumphantly to design high-performance ambipolar organic materials, novel molecular construction engineering on the basis of rigorous theory is still critically required to achieve reasonable, logical and ambipolar molecular structure design; iv) a great number of ambipolar organic semiconductors exist in the form of crystalline and hence display severe anisotropic ambipolar behavior, which is adverse for their large-area manufacture operation, that is, amorphous ambipolar semiconducting materials with large hole and electron mobilities as well as isotropic carrier transport characteristics are indispensable and favorable for low-cost fabrication process; v) in consideration of their practical applications, the operational and ambient stability of organic ambipolar transistors require further promotion. The air instability of organic materials is usually attributed 
to the charge trapping effect resulted from water and oxygen in air atmosphere, which can be tackled by lowering LUMO level of the organic semiconductors; vi) high annealing temperature is commonly required to form organic semiconducting thin films with better crystallinity and thereby exhibit highperformance ambipolar carrier transport. Whereas, this process gives rise to intricate manufacture operation and augmented power dissipation and hence new low-temperature annealing approach is eager to be investigated for realizing low-cost and flexible organic electronics; vii) the fabrication of ambipolar organic semiconductors based on solution-processing method with eco-friendly solvents is advantageous to decline laboratory pollution.

As to 2D semiconducting materials, both the ultrahigh charge transport capability and the high on/off ratio are attractive features for utilizing 2D materials in ambipolar transistors. However, there are still some challenges for exploiting 2D material-based ambipolar transistors from researches to applications: i) up to now, most ambipolar transistors are realized by complex modulation techniques (e.g., electric doublelayer gate), which will significantly increase fabrication complexities and result in integration difficulties. How to achieve ambipolar behavior based on 2D materials via simple modulation methods, e.g. easy chemical doping or special atmosphere annealing for 2D materials, is still great issue for 2D materialbased transistors. Therefore, simple, facile and low-cost modulation approaches should be developed as ever; ii) how to achieve ambipolar behaviors of 2D materials based on low-cost and large-area manufacture techniques, such as CVD, molecular beam epitaxy (MBE), and electronic paste, is also a significant challenge. Most 2D materials are fabricated by mechanically exfoliated method, which possesses several disadvantages for instance complicated transfer process, high cost and difficulty in acquiring large-area films. Thus, 2D material films based on low-cost and large-area fabrication techniques should be further investigated to fabricate ambipolar transistors; iii) how to construct flexible 2D material-based ambipolar transistor with good performance is a tremendous task. The flexible ambipolar transistor is attractive and stimulated to develop. However, the research of this aspect is rare; iv) simple and efficient fabrication methods should be exploited for stacking vdW heterostructures in ambipolar transistors; v) apart from traditional inorganic 2D material systems, such as TMDs and BP, novel organic and organic-inorganic hybrid 2D materials, such as COFs, CPs, and perovskites, can be focused in ambipolar transistors. In conclusion, further efforts should be devoted to developing 2D material-based ambipolar transistors with low cost, large-area fabrication, simple manufacture process, high integration, stable flexibility, and abundant material systems.

The well-balanced mobilities of both two types of charge carriers are expected for OIHPMs and OIDL transistors due to their special molecular structures and tunable material combinations. Furthermore, OIHPMs and OIDL transistors are also appropriate for flexible electronics on account of their solution processability and flexible fabrication approach. However, some challenges of OIHPMs and OIDL transistors still exist and should be overcome: i) the charge mobilities of both OIHPMs and OIDL transistors are still low; ii) the ambient stability of both materials and devices should be improved. Future efforts could be paid in two sides. On the one hand, molecular structures of materials should be further carefully designed to improve their mobility values and ambient stability. For example, introduction of inorganic cations (for OIHPMs) or organic groups (for polymers) is an effective method to achieve steady materials. On the other hand, various material combinations (e.g., introduction of nanowires, nanotubes, nanosheets and nanorods into organic or inorganic layer) and device structures can also be employed to improve charge mobilities.

With respect to functional ambipolar memories, large memory window, multilevel storage, fast switching speed, low potentiating bias as well as good endurance and retention capabilities have been vastly investigated and improved to conform to the request of their potential applications. For the sake of further commercialization and industrialization of ambipolar memories, several challenges together with their solutions are pointed out as follows: on one hand, their electronic performance still needs improving: i) switching speed: maximum switching speed of ambipolar memory devices is in direct connection with the electron and hole mobilities of semiconducting layer which are related to the intrinsic structures and properties of semiconductors as well as the interface contacts between semiconductors and blocking dielectric/source-drain electrodes. By modulating these parameters, improved transport mobilities of charges can be realized; ii) energy dissipation: the energy consumption of devices mainly relies on the operation voltage and thereby relatively thin blocking dielectric with large dielectric constant as well as novel high-performance semiconducting materials can be utilized to lower the potentiating amplitude $(\leq 1 \mathrm{~V})$ for addressing this limitation; iii) retention and endurance properties: to satisfy the requirements of practical flash memories, long-term and stable data retention as well as reliable repetitive program-erase ability are urgently demanded and air-stable ambipolar semiconducting materials, appropriate tunneling dielectric together with well-designed floating gate with steady trapping sites both are beneficial for this issue. On the other hand, investigation of flexible, stretchable, bendable and foldable ambipolar flash memories is essential for developing flexible displays and electronics and hence the mechanical flexibility under repeated tensile and compressive strains should be further researched.

For ambipolar synaptic transistors: i) how to realize linear and symmetric channel conductance change under consecutive electrical pulse trains for highly efficient learning remains challenging. By optimizing the transistor designs (structure, material, concentration, etc.) as well as the pulse parameters (pulse width, potentiating voltage, etc.) may lead to linear tuning of conductance; ii) the performance inconsistencies of various devices and different test cycles originating from inconclusive microstructures of fabricated semiconducting films are also a severe obstacle for neuromorphic computing, therefore, novel fabrication techniques and stable materials need to be explored; iii) large on/off ratio of ambipolar transistors is required to insure distinguishable multiple conductance levels and prevent the misreading due to conductance fluctuations; iv) integration of fundamental synaptic devices is capable of emulating complex neural functions such as image recognition but the manufacture complexity as well as power consumption are increased, which thus requires future research efforts on stack 
and integration capability and power efficiency of neural electronic systems.

In regard to ambipolar CMOS inverters, well-balanced large mobilities and symmetric turn-on voltages of holes and electrons as well as relatively small off current (hence large on/ off ratio) of ambipolar transistors are both important for their better performance such as low energy dissipation, high gain and noise immunity, etc. However, how to operate under unipolar mode and selectively transport unipolar charges with aforementioned favorable conditions remains sufficiently challenging. Several strategies such as intercalation of carrier injection layers, utilization of SAMs, chemical doping, etc., have been proposed and more effective methods are expected.

Finally, with regard to ambipolar LETs: i) the fundamental principles of carrier movement, exciton recombination and thereby light emission within semiconductor are still not fully understood in spite of continuous emerging novel materials and structures for high-performance light-emitting devices and deeper comprehending of underlying mechanisms is necessary; ii) most researches about ambipolar LETs just settle one or some characteristics of them, which is unilateral and difficult to achieve commercial uses (displays, solid-state lighting, electrically driven organic lasers, etc.). Apparently, overall improvements of diverse kinds of qualities of LETs are more favorable for practical applications in comparison with conventional OLEDs. Thus, combination of multiple reported approaches to increase performance together may figure out this problem to some extent. Meanwhile, more simple, low-cost, effective fabrication methods also need to be further studied; iii) despite the fact that ambipolar LETs usually exhibit much larger lightemission efficiency compared to their unipolar counterparts, light-emitting characteristics of ambipolar devices may be inappropriate for being utilized in active matrix displays on account of the reason that the light-emission strength of them rests with not only drain current but also other factors. Therefore, new emerging device structures for instance vertical and electrolyte-gated LETs have provided attractive features for constructing high-performance LETs.

For future perspective, ambipolar transistors based on organic polymers and 2D materials with higher mobility and on/off ratio are expected and combinations of high electrical performance, facile and low-cost fabrication method, low operation voltage (low power consumption) as well as good flexibility in single ambipolar transistor require future research in order to realize practical applications of ambipolar transistors. In addition, ambipolar synaptic transistors are also promising future research directions because they can be reconfigured between excitatory and inhibitory modes and hence achieve dynamic tunable synaptic plasticity, which finally is capable of overcoming conventional von Neumann bottleneck. Furthermore, the valley degree of freedom within the momentum space in TMDs is able to be regulated and utilized to carry information. The generated excitons reserve the valley-spin information and thereby light emitters relying on valley are able to be achieved. Besides, the circularly polarized emission in TMDs can be controlled by electric field. Further comprehension of the mechanisms of circular polarized electroluminescence as well as the realization of electroluminescence polarization under room temperature both are extremely important future subjects.
On the whole, ambipolar transistors with numerous advantages and prospective applications are under extensive and rapid research progress and the commercialization will emerge soon via tackling aforementioned technical challenges.

\section{Acknowledgements}

Y.R. and X.Y. contributed equally to this work. The authors acknowledge the grants from Natural Science Foundation of China (Grant No. 61604097), Guangdong Province Special Support Plan for HighLevel Talents (Grant No. 2017TQ04X082), Guangdong Provincial Department of Science and Technology (Grant No. 2018B030306028), the Science and Technology Innovation Commission of Shenzhen (Grant Nos. JCYJ20180507182042530, JCYJ20180507182000722, and JCYJ20170818143618288), Shenzhen Peacock Technological Innovation Project (Grant Nos. KQJSCX20170727100433270 and KQJSCX20170327150812967), and the Natural Science Foundation of SZU.

\section{Conflict of Interest}

The authors declare no conflict of interest.

\section{Keywords}

2D material, ambipolar memory, ambipolar synapse, ambipolar transistor, organic semiconductor

Received: March 11, 2019

Revised: July 8, 2019

Published online: July 26, 2019

[1] M. Muccini, Nat. Mater. 2006, 5, 605 .

[2] P. Heremans, A. K. Tripathi, A. de Jamblinne de Meux, E. C. Smits, B. Hou, G. Pourtois, G. H. Gelinck, Adv. Mater. 2016, 28, 4266.

[3] X. Guo, Y. Xu, S. Ogier, T. N. Ng, M. Caironi, A. Perinot, L. Li, J. Zhao, W. Tang, R. A. Sporea, A. Nejim, J. Carrabina, P. Cain, F. Yan, IEEE Trans. Electron Devices 2017, 64, 1906.

[4] X. Ren, F. Yang, X. Gao, S. Cheng, X. Zhang, H. Dong, W. Hu, Adv. Energy Mater. 2018, 8, 1801003.

[5] Y. Zhao, K. Xu, F. Pan, C. Zhou, F. Zhou, Y. Chai, Adv. Funct. Mater. 2017, 27, 1603484

[6] H. Sirringhaus, Adv. Mater. 2014, 26, 1319.

[7] L. Dou, Y. Liu, Z. Hong, G. Li, Y. Yang, Chem. Rev. 2015, 115, 12633.

[8] H. Dong, X. Fu, J. Liu, Z. Wang, W. Hu, Adv. Mater. 2013, 25, 6158.

[9] Y. Olivier, D. Niedzialek, V. Lemaur, W. Pisula, K. Mullen, U. Koldemir, J. R. Reynolds, R. Lazzaroni, J. Cornil, D. Beljonne, Adv. Mater. 2014, 26, 2119.

[10] S. Z. Bisri, C. Piliego, J. Gao, M. A. Loi, Adv. Mater. 2014, 26, 1176.

[11] H. Hwang, D. Khim, J.-M. Yun, E. Jung, S.-Y. Jang, Y. H. Jang, Y.-Y. Noh, D.-Y. Kim, Adv. Funct. Mater. 2015, 25, 1146.

[12] Z. Ni, H. Wang, Q. Zhao, J. Zhang, Z. Wei, H. Dong, W. Hu, Adv. Mater. 2019, 31, 1806010.

[13] B. W. Baugher, H. O. Churchill, Y. Yang, P. Jarillo-Herrero, Nat. Nanotechnol. 2014, 9, 262.

[14] F. Li, C. Ma, H. Wang, W. Hu, W. Yu, A. D. Sheikh, T. Wu, Nat. Commun. 2015, 6, 8238.

[15] Y. Zhao, Y. Guo, Y. Liu, Adv. Mater. 2013, 25, 5372. 
[16] H. C. Chang, C. Lu, C. L. Liu, W. C. Chen, Adv. Mater. 2015, 27, 27.

[17] H. Tian, Q. Guo, Y. Xie, H. Zhao, C. Li, J. J. Cha, F. Xia, H. Wang, Adv. Mater. 2016, 28, 4991.

[18] H. Pfleiderer, W. Kusian, Solid-State Electron. 1986, $29,317$.

[19] C. van Berkel, M. J. Powell, Appl. Phys. Lett. 1987, 51, 1094.

[20] T. Yasuda, T. Goto, K. Fujita, T. Tsutsui, Appl. Phys. Lett. 2004, 85, 2098.

[21] E. J. Meijer, D. M. de Leeuw, S. Setayesh, E. van Veenendaal, B. H. Huisman, P. W. Blom, J. C. Hummelen, U. Scherf, J. Kadam, T. M. Klapwijk, Nat. Mater. 2003, 2, 678.

[22] H. C. Pao, C. T. Sah, Solid-State Electron. 1966, 9, 927.

[23] G. Paasch, T. Lindner, C. Rost-Bietsch, S. Karg, W. Riess, S. Scheinert, J. Appl. Phys. 2005, 98, 084505.

[24] R. Schmechel, M. Ahles, H. von Seggern, J. Appl. Phys. 2005, 98, 084511.

[25] C. Zhu, Z. Zhao, H. Chen, L. Zheng, X. Li, J. Chen, Y. Sun, F. Liu, Y. Guo, Y. Liu, J. Am. Chem. Soc. 2017, 139, 17735.

[26] S.-S. Cheng, P.-Y. Huang, M. Ramesh, H.-C. Chang, L.-M. Chen, C.-M. Yeh, C.-L. Fung, M.-C. Wu, C.-C. Liu, C. Kim, H.-C. Lin, M.-C. Chen, C.-W. Chu, Adv. Funct. Mater. 2014, 24, 2057.

[27] D. Gao, X. Zhang, X. Kong, Y. Chen, J. Jiang, ACS Appl. Mater. Interfaces 2015, 7, 2486.

[28] D. Natali, M. Caironi, Adv. Mater. 2012, 24, 1357.

[29] H. Ishii, K. Sugiyama, E. Ito, K. Seki, Adv. Mater. 1999, 11, 605.

[30] S. Fabiano, H. Yoshida, Z. Chen, A. Facchetti, M. A. Loi, ACS Appl. Mater. Interfaces 2013, 5, 4417.

[31] D. M. deLeeuw, M. M. J. Simenon, A. R. Brown, R. E. F. Einerhand, Synth. Met. 1997, 87, 53.

[32] H. N. Tsao, K. Mullen, Chem. Soc. Rev. 2010, 39, 2372.

[33] M. Mas-Torrent, C. Rovira, Chem. Rev. 2011, 111, 4833.

[34] J. Lee, A. R. Han, J. Kim, Y. Kim, J. H. Oh, C. Yang, J. Am. Chem. Soc. 2012, 134, 20713.

[35] I. Tszydel, M. Kucinska, T. Marszalek, R. Rybakiewicz, A. Nosal, J. Jung, M. Gazicki-Lipman, C. Pitsalidis, C. Gravalidis, S. Logothetidis, M. Zagorska, J. Ulanski, Adv. Funct. Mater. 2012, 22, 3840 .

[36] H. Klauk, Chem. Soc. Rev. 2010, 39, 2643.

[37] X. Sun, Y. Liu, C. A. Di, Y. Wen, Y. Guo, L. Zhang, Y. Zhao, G. Yu, Adv. Mater. 2011, 23, 1009

[38] T. Kanagasekaran, H. Shimotani, S. Ikeda, H. Shang, R. Kumashiro, K. Tanigaki, Appl. Phys. Lett. 2015, 107, 043304.

[39] I. Cho, S. K. Park, B. Kang, J. W. Chung, J. H. Kim, W. S. Yoon, K. Cho, S. Y. Park, J. Mater. Chem. C 2016, 4, 9460.

[40] M. Ozdemir, D. Choi, G. Kwon, Y. Zorlu, H. Kim, M.-G. Kim, S. Seo, U. Sen, M. Citir, C. Kim, H. Usta, RSC Adv. 2016, 6, 212.

[41] Q. Shuai, H. T. Black, A. Dadvand, D. F. Perepichka, J. Mater. Chem. C 2014, 2, 3972.

[42] Y. Qin, C. Cheng, H. Geng, C. Wang, W. Hu, W. Xu, Z. Shuai, D. Zhu, Phys. Chem. Chem. Phys. 2016, 18, 14094.

[43] F. Zhang, Y. Hu, T. Schuettfort, C. A. Di, X. Gao, C. R. McNeill, L. Thomsen, S. C. Mannsfeld, W. Yuan, H. Sirringhaus, D. Zhu, J. Am. Chem. Soc. 2013, 135, 2338.

[44] K. Liu, C.-L. Song, Y.-C. Zhou, X.-Y. Zhou, X.-J. Pan, L.-Y. Cao, C. Zhang, Y. Liu, X. Gong, H.-L. Zhang, J. Mater. Chem. C 2015, 3, 4188.

[45] K. Zhou, H. Dong, H. L. Zhang, W. Hu, Phys. Chem. Chem. Phys. 2014, 16, 22448.

[46] A. Riaño, P. Mayorga Burrezo, M. J. Mancheño, A. Timalsina, J. Smith, A. Facchetti, T. J. Marks, J. T. López Navarrete, J. L. Segura, J. Casado, R. Ponce Ortiz, J. Mater. Chem. C 2014, 2, 6376.

[47] X. Chen, G. Zhang, H. Luo, Y. Li, Z. Liu, D. Zhang, J. Mater. Chem. C 2014, 2, 2869.

[48] E. Wang, W. Mammo, M. R. Andersson, Adv. Mater. 2014, 26, 1801.
[49] H. Luo, X. Dong, Z. Cai, L. Wang, Z. Liu, Chem. - Asian J. 2018, 7, 592.

[50] Z. Cai, H. Luo, X. Chen, G. Zhang, Z. Liu, D. Zhang, Chem. - Asian J. 2014, 9, 1068.

[51] J. Bai, Y. Liu, S. Oh, W. Lei, B. Yin, S. Park, Y. Kan, RSC Adv. 2015, 5, 53412.

[52] A. Diac, D. Demeter, M. Allain, I. Grosu, J. Roncali, Chem. - Eur. J. 2015, 21, 1598.

[53] Y. Fukutomi, M. Nakano, J. Y. Hu, I. Osaka, K. Takimiya, J. Am. Chem. Soc. 2013, 135, 11445.

[54] G. Lin, Y. Qin, Y.-S. Guan, H. Xu, W. Xu, D. Zhu, RSC Adv. 2016, 6, 4872.

[55] I. Meager, M. Nikolka, B. C. Schroeder, C. B. Nielsen, M. Planells, H. Bronstein, J. W. Rumer, D. I. James, R. S. Ashraf, A. Sadhanala, P. Hayoz, J.-C. Flores, H. Sirringhaus, I. McCulloch, Adv. Funct. Mater. 2014, 24, 7109.

[56] Y. Qiao, Y. Guo, C. Yu, F. Zhang, W. Xu, Y. Liu, D. Zhu, J. Am. Chem. Soc. 2012, 134, 4084.

[57] Q. Wu, S. Ren, M. Wang, X. Qiao, H. Li, X. Gao, X. Yang, D. Zhu, Adv. Funct. Mater. 2013, 23, 2277.

[58] J. D. Yuen, J. Fan, J. Seifter, B. Lim, R. Hufschmid, A. J. Heeger, F. Wudl, J. Am. Chem. Soc. 2011, 133, 20799.

[59] Y. Zhang, C. Kim, J. Lin, T.-Q. Nguyen, Adv. Funct. Mater. 2012, 22, 97.

[60] G. Lin, Y. Qin, J. Zhang, Y.-S. Guan, H. Xu, W. Xu, D. Zhu, J. Mater. Chem. C 2016, 4, 4470.

[61] M. J. Kim, M. Jung, W. Kang, G. An, H. Kim, H. J. Son, B. Kim, J. H. Cho, J. Phys. Chem. C 2015, 119, 16414.

[62] Z. Wang, X. Li, Y. Zou, J. Tan, X. Fu, J. Liu, C. Xiao, H. Dong, W. Jiang, F. Liu, Y. Zhen, Z. Wang, T. P. Russell, W. Hu, J. Mater. Chem. C 2016, 4, 7230.

[63] R. Ozdemir, D. Choi, M. Ozdemir, G. Kwon, H. Kim, U. Sen, C. Kim, H. Usta, J. Mater. Chem. C 2017, 5, 2368.

[64] J.-Y. Hu, M. Nakano, I. Osaka, K. Takimiya, J. Mater. Chem. C 2015, 3, 4244.

[65] I. V. Klimovich, L. I. Leshanskaya, S. I. Troyanov, D. V. Anokhin, D. V. Novikov, A. A. Piryazev, D. A. Ivanov, N. N. Dremova, P. A. Troshin, J. Mater. Chem. C 2014, 2, 7621.

[66] O. Pitayatanakul, T. Higashino, T. Kadoya, M. Tanaka, H. Kojima, M. Ashizawa, T. Kawamoto, H. Matsumoto, K. Ishikawa, T. Mori, J. Mater. Chem. C 2014, 2, 9311.

[67] O. Pitayatanakul, K. lijima, M. Ashizawa, T. Kawamoto, H. Matsumoto, T. Mori, J. Mater. Chem. C 2015, 3, 8612.

[68] M. Ashizawa, N. Masuda, T. Higashino, T. Kadoya, T. Kawamoto, H. Matsumoto, T. Mori, Org. Electron. 2016, 35, 95

[69] W. Yue, T. He, M. Stolte, M. Gsanger, F. Wurthner, Chem. Commun. 2014, 50, 545.

[70] D. Yoo, T. Hasegawa, M. Ashizawa, T. Kawamoto, H. Masunaga, T. Hikima, H. Matsumoto, T. Mori, J. Mater. Chem. C 2017, 5, 2509.

[71] G. E. Rudebusch, J. L. Zafra, K. Jorner, K. Fukuda, J. L. Marshall, I. Arrechea-Marcos, G. L. Espejo, R. Ponce Ortiz, C. J. Gomez-Garcia, L. N. Zakharov, M. Nakano, H. Ottosson, J. Casado, M. M. Haley, Nat. Chem. 2016, 8, 753.

[72] J. Guo, D. Liu, J. Zhang, J. Zhang, Q. Miao, Z. Xie, Chem. Commun. 2015, 51, 12004.

[73] H. Xu, Y.-C. Zhou, X.-Y. Zhou, K. Liu, L.-Y. Cao, Y. Ai, Z.-P. Fan, H.-L. Zhang, Adv. Funct. Mater. 2014, 24, 2907.

[74] M. Kang, H. Hwang, W. T. Park, D. Khim, J. S. Yeo, Y. Kim, Y. J. Kim, Y. Y. Noh, D. Y. Kim, ACS Appl. Mater. Interfaces 2017, 9, 2686.

[75] S. Vegiraju, C.-Y. Lin, P. Priyanka, D.-Y. Huang, X.-L. Luo, H.-C. Tsai, S.-H. Hong, C.-J. Yeh, W.-C. Lien, C.-L. Wang, S.-H. Tung, C.-L. Liu, M.-C. Chen, A. Facchetti, Adv. Funct. Mater. 2018, 28, 1801025. 
[76] J. Zhang, W. Xu, P. Sheng, G. Zhao, D. Zhu, Acc. Chem. Res. 2017 50, 1654.

[77] H. T. Black, D. F. Perepichka, Angew. Chem., Int. Ed. 2014, 53, 2138.

[78] C. Fujisue, T. Kadoya, T. Higashino, R. Sato, T. Kawamoto, T. Mori, RSC Adv. 2016, 6, 53345.

[79] Y. Qin, J. Zhang, X. Zheng, H. Geng, G. Zhao, W. Xu, W. Hu, Z. Shuai, D. Zhu, Adv. Mater. 2014, 26, 4093

[80] X. Xu, T. Xiao, X. Gu, X. Yang, S. V. Kershaw, N. Zhao, J. Xu, Q. Miao, ACS Appl. Mater. Interfaces 2015, 7, 28019.

[81] J. Deng, Y. Xu, L. Liu, C. Feng, J. Tang, Y. Gao, Y. Wang, B. Yang, P. Lu, W. Yang, Y. Ma, Chem. Commun. 2016, 52, 2370.

[82] M. Jea, A. Kumar, H. Cho, D. Yang, H. Shim, A. K. Palai, S. Pyo, J. Mater. Chem. C 2014, 2, 3980.

[83] T. Kushida, S. Shirai, N. Ando, T. Okamoto, H. Ishii, H. Matsui, M. Yamagishi, T. Uemura, J. Tsurumi, S. Watanabe, J. Takeya, S. Yamaguchi, J. Am. Chem. Soc. 2017, 139, 14336.

[84] S. Mu, K. Oniwa, T. Jin, N. Asao, M. Yamashita, S. Takaishi, Org. Electron. 2016, 34, 23

[85] J. C. Ribierre, L. Zhao, S. Furukawa, T. Kikitsu, D. Inoue, A. Muranaka, K. Takaishi, T. Muto, S. Matsumoto, D. Hashizume, M. Uchiyama, P. Andre, C. Adachi, T. Aoyama, Chem. Commun. 2015, 51, 5836.

[86] M. Pope, C. E. Swenberg, Electronic Processes in Organic Crystals and Polymers, Oxford University Press, New York 1999.

[87] R. Farchioni, G. Grosso, Organic Electronic Materials: Conjugated Polymers and Low Molecular Weight Organic Solids, Springer, Berlin 2001.

[88] N. Toyota, M. Lang, J. Mueller, Low-Dimensional Molecular Metals, Springer, Berlin 2007.

[89] Y. Zhang, H. Dong, Q. Tang, S. Ferdous, F. Liu, S. C. Mannsfeld, W. Hu, A. L. Briseno, J. Am. Chem. Soc. 2010, 132, 11580.

[90] T. He, M. Stolte, F. Wurthner, Adv. Mater. 2013, 25, 6951.

[91] Z. Chen, H. Lemke, S. Albert-Seifried, M. Caironi, M. M. Nielsen, M. Heeney, W. Zhang, I. McCulloch, H. Sirringhaus, Adv. Mater. $2010,22,2371$.

[92] H. Minemawari, T. Yamada, H. Matsui, J. Tsutsumi, S. Haas, R. Chiba, R. Kumai, T. Hasegawa, Nature 2011, 475, 364

[93] J. Jang, S. Nam, K. Im, J. Hur, S. N. Cha, J. Kim, H. B. Son, H. Suh, M. A. Loth, J. E. Anthony, J.-J. Park, C. E. Park, J. M. Kim, K. Kim, Adv. Funct. Mater. 2012, 22, 1005.

[94] S. Watanabe, T. Fujita, J. C. Ribierre, K. Takaishi, T. Muto, C. Adachi, M. Uchiyama, T. Aoyama, M. Matsumoto, ACS Appl. Mater. Interfaces 2016, 8, 17574

[95] A. Saeki, S. Seki, T. Takenobu, Y. Iwasa, S. Tagawa, Adv. Mater. 2008, 20, 920.

[96] O. D. Jurchescu, M. Popinciuc, B. J. van Wees, T. T. M. Palstra, Adv. Mater. 2007, 19, 688.

[97] J. Takeya, M. Yamagishi, Y. Tominari, R. Hirahara, Y. Nakazawa, T. Nishikawa, T. Kawase, T. Shimoda, S. Ogawa, Appl. Phys. Lett. 2007, 90, 102120.

[98] B. Peng, Z. Wang, P. K. L. Chan, J. Mater. Chem. C 2016, 4, 8628.

[99] Y. Yao, H. Dong, F. Liu, T. P. Russell, W. Hu, Adv. Mater. 2017, 29, 1701251.

[100] E. Menard, V. Podzorov, S. H. Hur, A. Gaur, M. E. Gershenson, J. A. Rogers, Adv. Mater. 2004, 16, 2097.

[101] K. Yamada, J. Takeya, T. Takenobu, Y. Iwasa, Appl. Phys. Lett. 2008, 92, 253311.

[102] Y. Krupskaya, M. Gibertini, N. Marzari, A. F. Morpurgo, Adv. Mater. 2015, 27, 2453.

[103] M. Chu, J. X. Fan, S. Yang, D. Liu, C. F. Ng, H. Dong, A. M. Ren, Q. Miao, Adv. Mater. 2018, 30, 1803467.

[104] R. W. I. de Boer, A. F. Stassen, M. F. Craciun, C. L. Mulder, A. Molinari, S. Rogge, A. F. Morpurgo, Appl. Phys. Lett. 2005, 86, 262109.
[105] T. Takahashi, T. Takenobu, J. Takeya, Y. Iwasa, Appl. Phys. Lett. 2006, 88, 033505

[106] S. Z. Bisri, T. Takahashi, T. Takenobu, M. Yahiro, C. Adachi, Y. Iwasa, Jpn. J. Appl. Phys. 2007, 46, L596.

[107] S. Z. Bisri, T. Takenobu, Y. Yomogida, H. Shimotani, T. Yamao S. Hotta, Y. Iwasa, Adv. Funct. Mater. 2009, 19, 1728.

[108] T. Takahashi, T. Takenobu, J. Takeya, Y. Iwasa, Adv. Funct. Mater. 2007, 17, 1623.

[109] T. Takenobu, K. Watanabe, Y. Yomogida, H. Shimotani, Y. Iwasa, Appl. Phys. Lett. 2008, 93, 073301.

[110] S. Z. Bisri, T. Takenobu, T. Takahashi, Y. Iwasa, Appl. Phys. Lett. 2010, 96, 183304

[111] T. Uemura, M. Yamagishi, Y. Okada, K. Nakayama, M. Yoshizumi, M. Uno, J. Takeya, Adv. Mater. 2010, 22, 3938.

[112] S. Z. Bisri, K. Sawabe, M. Imakawa, K. Maruyama, T. Yamao, S. Hotta, Y. Iwasa, T. Takenobu, Sci. Rep. 2012, 2, 985.

[113] S. Z. Bisri, T. Takenobu, K. Sawabe, S. Tsuda, Y. Yomogida, T. Yamao, S. Hotta, C. Adachi, Y. Iwasa, Adv. Mater. 2011, 23, 2753.

[114] L. L. Chua, J. Zaumseil, J. F. Chang, E. C. W. Ou, P. K. H. Ho, H. Sirringhaus, R. H. Friend, Nature 2005, 434, 194.

[115] K. Sawabe, T. Takenobu, S. Z. Bisri, T. Yamao, S. Hotta, Y. Iwasa, Appl. Phys. Lett. 2010, 97, 043307.

[116] H. Nakanotani, M. Saito, H. Nakamura, C. Adachi, Appl. Phys. Lett. 2009, 95, 033308.

[117] K. Sawabe, M. Imakawa, M. Nakano, T. Yamao, S. Hotta, Y. Iwasa, T. Takenobu, Adv. Mater. 2012, 24, 6141.

[118] S. K. Park, S. Varghese, J. H. Kim, S. J. Yoon, O. K. Kwon, B. K. An, J. Gierschner, S. Y. Park, J. Am. Chem. Soc. 2013, 135, 4757.

[119] J. Zhang, J. Tan, Z. Ma, W. Xu, G. Zhao, H. Geng, C. Di, W. Hu, Z. Shuai, K. Singh, D. Zhu, J. Am. Chem. Soc. 2013, 135, 558.

[120] H. Shim, A. Kumar, H. Cho, D. Yang, A. K. Palai, S. Pyo, ACS Appl. Mater. Interfaces 2014, 6, 17804.

[121] T. Kanagasekaran, H. Shimotani, R. Shimizu, T. Hitosugi, K. Tanigaki, Nat. Commun. 2017, 8, 999.

[122] H. Jiang, K. J. Tan, K. K. Zhang, X. Chen, C. Kloc, J. Mater. Chem. 2011, 21, 4771

[123] A. L. Briseno, S. C. Mannsfeld, M. M. Ling, S. Liu, R. J. Tseng, C. Reese, M. E. Roberts, Y. Yang, F. Wudl, Z. Bao, Nature 2006, 444, 913.

[124] A. Dodabalapur, H. E. Katz, L. Torsi, R. C. Haddon, Appl. Phys. Lett. 1996, 68, 1108.

[125] Y. Wu, P. Ma, N. Wu, X. Kong, M. Bouvet, X. Li, Y. Chen, J. Jiang, Adv. Mater. Interfaces 2016, 3, 1600253.

[126] S. D. Wang, K. Kanai, Y. Ouchi, K. Seki, Org. Electron. 2006, 7, 457.

[127] K. Eguchi, M. M. Matsushita, K. Awaga, J. Phys. Chem. C 2018, 122, 26054.

[128] S. M. Obaidulla, P. K. Giri, J. Mater. Chem. C 2015, 3, 7118.

[129] L.-K. Mao, J.-C. Hwang, Y.-L. Chueh, Org. Electron. 2014, 15, 2400.

[130] S. Vasimalla, N. V. V. Subbarao, P. K. Iyer, J. Mater. Chem. C 2016, 4, 7102.

[131] J.-F. Chang, W.-R. Chen, S.-M. Huang, Y.-C. Lai, X.-Y. Lai, Y.-W. Yang, C.-H. Wang, Org. Electron. 2015, $27,84$.

[132] S. Cho, J. Yuen, J. Y. Kim, K. Lee, A. J. Heeger, S. Lee, Appl. Phys. Lett. 2008, 92, 063505.

[133] Q. Wei, K. Tajima, K. Hashimoto, ACS Appl. Mater. Interfaces 2009, 1, 1865.

[134] F. S. Kim, E. Ahmed, S. Subramaniyan, S. A. Jenekhe, ACS Appl. Mater. Interfaces 2010, 2, 2974.

[135] X. Zhao, T. Liu, H. Liu, S. Wang, X. Li, Y. Zhang, X. Hou, Z. Liu, W. Shi, T. J. S. Dennis, ACS Appl. Mater. Interfaces 2018, 10, 42715.

[136] T. Lei, J. H. Dou, X. Y. Cao, J. Y. Wang, J. Pei, J. Am. Chem. Soc. 2013, 135, 12168

[137] G. Kim, S. J. Kang, G. K. Dutta, Y. K. Han, T. J. Shin, Y. Y. Noh, C. Yang, J. Am. Chem. Soc. 2014, 136, 9477. 
[138] W. Yue, R. S. Ashraf, C. B. Nielsen, E. Collado-Fregoso, M. R. Niazi, S. A. Yousaf, M. Kirkus, H. Y. Chen, A. Amassian, J. R. Durrant, I. McCulloch, Adv. Mater. 2015, 27, 4702.

[139] S. Li, Z. Yuan, J. Yuan, P. Deng, Q. Zhang, B. Sun, J. Mater. Chem. A 2014, 2, 5427.

[140] H. Lin, S. Chen, Z. Li, J. Y. Lai, G. Yang, T. McAfee, K. Jiang, Y. Li, Y. Liu, H. Hu, J. Zhao, W. Ma, H. Ade, H. Yan, Adv. Mater. 2015, 27, 7299.

[141] B. Wang, T. P. Huynh, W. Wu, N. Hayek, T. T. Do, J. C. Cancilla, J. S. Torrecilla, M. M. Nahid, J. M. Colwell, O. M. Gazit, S. R. Puniredd, C. R. McNeill, P. Sonar, H. Haick, Adv. Mater. 2016, $28,4012$.

[142] Y. Xu, H. Sun, E. Y. Shin, Y. F. Lin, W. Li, Y. Y. Noh, Adv. Mater. 2016, 28, 8531

[143] H. R. Tseng, H. Phan, C. Luo, M. Wang, L. A. Perez, S. N. Patel, L. Ying, E. J. Kramer, T. Q. Nguyen, G. C. Bazan, A. J. Heeger, Adv. Mater. 2014, 26, 2993.

[144] C.-J. Chiang, J.-C. Chen, H.-Y. Tsao, K.-Y. Wu, C.-L. Wang, Adv. Funct. Mater. 2015, 25, 606.

[145] X. Qiao, Q. Wu, H. Wu, J. Zhang, H. Li, Adv. Funct. Mater. 2017, 27, 1604286.

[146] S. Shi, H. Wang, P. Chen, M. A. Uddin, Y. Wang, Y. Tang, H. Guo, X. Cheng, S. Zhang, H. Y. Woo, X. Guo, Polym. Chem. 2018, 9, 3873.

[147] Y. Wang, T. Hasegawa, H. Matsumoto, T. Mori, T. Michinobu, Adv. Funct. Mater. 2017, 27, 1604608.

[148] J. Fan, J. D. Yuen, M. Wang, J. Seifter, J. H. Seo, A. R. Mohebbi, D. Zakhidov, A. Heeger, F. Wudl, Adv. Mater. 2012, 24, 2186.

[149] Z. Cai, H. Luo, P. Qi, J. Wang, G. Zhang, Z. Liu, D. Zhang, Macromolecules 2014, 47, 2899.

[150] M. Jang, J. H. Kim, D. H. Hwang, H. Yang, ACS Appl. Mater. Interfaces 2015, 7, 12781

[151] C. An, M. Li, T. Marszalek, D. Li, R. Berger, W. Pisula, M. Baumgarten, Chem. Mater. 2014, 26, 5923.

[152] D. Venkateshvaran, M. Nikolka, A. Sadhanala, V. Lemaur, M. Zelazny, M. Kepa, M. Hurhangee, A. J. Kronemeijer, V. Pecunia, I. Nasrallah, I. Romanov, K. Broch, I. McCulloch, D. Emin, Y. Olivier, J. Cornil, D. Beljonne, H. Sirringhaus, Nature 2014, 515, 384.

[153] G. Zhang, P. Li, L. Tang, J. Ma, X. Wang, H. Lu, B. Kang, K. Cho, L. Qiu, Chem. Commun. 2014, 50, 3180 .

[154] J. Yang, Y. Jiang, Z. Tu, Z. Zhao, J. Chen, Z. Yi, Y. Li, S. Wang, Y. Yi, Y. Guo, Y. Liu, Adv. Funct. Mater. 2019, 29, 1804839.

[155] Z. Yi, S. Wang, Y. Liu, Adv. Mater. 2015, 27, 3589.

[156] C. B. Nielsen, M. Turbiez, I. McCulloch, Adv. Mater. 2013, 25, 1859.

[157] H. Chen, Y. Guo, Z. Mao, G. Yu, J. Huang, Y. Zhao, Y. Liu, Chem. Mater. 2013, 25, 3589.

[158] X. Guo, F. S. Kim, M. J. Seger, S. A. Jenekhe, M. D. Watson, Chem. Mater. 2012, 24, 1434.

[159] Y. Kim, J. Hong, J. H. Oh, C. Yang, Chem. Mater. 2013, 25, 3251.

[160] M. Nakano, I. Osaka, K. Takimiya, Macromolecules 2015, 48, 576.

[161] Z. Zhao, Z. Yin, H. Chen, L. Zheng, C. Zhu, L. Zhang, S. Tan, H. Wang, Y. Guo, Q. Tang, Y. Liu, Adv. Mater. 2017, 29, 1602410.

[162] C. An, H. Makowska, B. Hu, R. Duan, W. Pisula, T. Marszalek, M. Baumgarten, RSC Adv. 2018, 8, 16464.

[163] Z. Liu, G. Zhang, Z. Cai, X. Chen, H. Luo, Y. Li, J. Wang, D. Zhang, Adv. Mater. 2014, 26, 6965

[164] R. Kim, P. S. K. Amegadze, I. Kang, H.--. Yun, Y.-Y. Noh, S.-K. Kwon, Y.-H. Kim, Adv. Funct. Mater. 2013, 23, 5719.

[165] T. Lei, J. Y. Wang, J. Pei, Acc. Chem. Res. 2014, 47, 1117.

[166] P. Deng, Q. Zhang, Polym. Chem. 2014, 5, 3298.

[167] R. Stalder, J. Mei, K. R. Graham, L. A. Estrada, J. R. Reynolds, Chem. Mater. 2014, 26, 664

[168] H. Hwang, Y. Kim, M. Kang, M.-H. Lee, Y.-J. Heo, D.-Y. Kim, Polym. Chem. 2017, 8, 361.
[169] R. Stalder, S. R. Puniredd, M. R. Hansen, U. Koldemir, C. Grand, W. Zajaczkowski, K. Müllen, W. Pisula, J. R. Reynolds, Chem. Mater. 2016, 28, 1286

[170] H.-C. Wu, C.-C. Hung, C.-W. Hong, H.-S. Sun, J.-T. Wang, G. Yamashita, T. Higashihara, W.-C. Chen, Macromolecules 2016, 49, 8540.

[171] J. Mei, K. R. Graham, R. Stalder, J. R. Reynolds, Org. Lett. 2010, 12, 660.

[172] T. Lei, Y. Cao, Y. Fan, C. J. Liu, S. C. Yuan, J. Pei, J. Am. Chem. Soc. 2011, 133, 6099

[173] Y. He, C. Guo, B. Sun, J. Quinn, Y. Li, Chem. Commun. 2015, 51, 8093.

[174] Y. Deng, B. Sun, Y. He, J. Quinn, C. Guo, Y. Li, Chem. Commun. 2015, 51, 13515

[175] T. Takaya, M. D. Mamo, M. Karakawa, Y.-Y. Noh, J. Mater. Chem. C 2018, 6, 7822

[176] G. Zhang, Z. Ye, P. Li, J. Guo, Q. Wang, L. Tang, H. Lu, L. Qiu, Polym. Chem. 2015, 6, 3970.

[177] Y. Q. Zheng, T. Lei, J. H. Dou, X. Xia, J. Y. Wang, C. J. Liu, J. Pei, Adv. Mater. 2016, 28, 7213.

[178] X. Zhou, N. Ai, Z.-H. Guo, F.-D. Zhuang, Y.-S. Jiang, J.-Y. Wang, J. Pei, Chem. Mater. 2015, 27, 1815.

[179] Y. Jiang, Y. Gao, H. Tian, J. Ding, D. Yan, Y. Geng, F. Wang, Macromolecules 2016, 49, 2135

[180] N. Zhao, N. Ai, M. Cai, X. Wang, J. Pei, X. Wan, Polym. Chem. 2016, 7, 235

[181] R. S. Ashraf, A. J. Kronemeijer, D. I. James, H. Sirringhaus, I. McCulloch, Chem. Commun. 2012, 48, 3939.

[182] W. Yue, M. Nikolka, M. Xiao, A. Sadhanala, C. B. Nielsen, A. J. P. White, H.-Y. Chen, A. Onwubiko, H. Sirringhaus, I. McCulloch, J. Mater. Chem. C 2016, 4, 9704.

[183] J. Huang, Z. Mao, Z. Chen, D. Gao, C. Wei, W. Zhang, G. Yu, Chem. Mater. 2016, 28, 2209.

[184] T. Lei, J. H. Dou, Z. J. Ma, C. H. Yao, C. J. Liu, J. Y. Wang, J. Pei, J. Am. Chem. Soc. 2012, 134, 20025.

[185] Y. Gao, Y. Deng, H. Tian, J. Zhang, D. Yan, Y. Geng, F. Wang, Adv. Mater. 2017, 29, 1606217

[186] J. Yang, Z. Zhao, H. Geng, C. Cheng, J. Chen, Y. Sun, L. Shi, Y. Yi, Z. Shuai, Y. Guo, S. Wang, Y. Liu, Adv. Mater. 2017, 29, 1702115.

[187] D. G. Parnum, G. Mehta, F. P. S. George, G. I. Moore, Tetrahedron Lett. 1974, 29, 2549.

[188] E. K. Lee, C. H. Park, J. Lee, H. R. Lee, C. Yang, J. H. Oh, Adv. Mater. 2017, 29, 1605282

[189] H. J. Yun, G. B. Lee, D. S. Chung, Y. H. Kim, S. K. Kwon, Adv. Mater. 2014, 26, 6612

[190] G. D. Tabi, B. Nketia-Yawson, J. Y. Lee, K. Cho, B. Lim, Y.-Y. Noh, RSC Adv. 2017, 7, 1110

[191] C. J. Mueller, C. R. Singh, M. Fried, S. Huettner, M. Thelakkat, Adv. Funct. Mater. 2015, 25, 2725.

[192] A. R. Han, G. K. Dutta, J. Lee, H. R. Lee, S. M. Lee, H. Ahn, T. J. Shin, J. H. Oh, C. Yang, Adv. Funct. Mater. 2015, 25, 247.

[193] T. Bura, S. Beaupré, O. A. Ibraikulov, M.-A. Légaré, J. Quinn, P. Lévêque, T. Heiser, Y. Li, N. Leclerc, M. Leclerc, Macromolecules 2017, 50, 7080.

[194] H. Zhang, K. Yang, K. Zhang, Z. Zhang, Q. Sun, W. Yang, Polym. Chem. 2018, 9, 1807.

[195] Z. Yi, Y. Jiang, L. Xu, C. Zhong, J. Yang, Q. Wang, J. Xiao, X. Liao, S. Wang, Y. Guo, W. Hu, Y. Liu, Adv. Mater. 2018, 30, 1801951.

[196] E. D. Glowacki, H. Coskun, M. A. Blood-Forsythe, U. Monkowius, L. Leonat, M. Grzybowski, D. Gryko, M. S. White, A. Aspuru-Guzik, N. S. Sariciftci, Org. Electron. 2014, 15, 3521.

[197] T. W. Lee, D. H. Lee, J. Shin, M. J. Cho, D. H. Choi, Polym. Chem. 2015, 6, 1777

[198] P. Sonar, J. Chang, J. H. Kim, K. H. Ong, E. Gann, S. Manzhos, J. Wu, C. R. McNeill, ACS Appl. Mater. Interfaces 2016, 8, 24325. 
[199] M. J. Kim, J. H. Park, B. Kang, D. Kim, A. R. Jung, J. Yang, M. S. Kang, D. Y. Lee, K. Cho, H. Kim, B. Kim, J. H. Cho, J. Phys. Chem. C 2016, 120, 13865.

[200] Z. Chen, D. Gao, J. Huang, Z. Mao, W. Zhang, G. Yu, ACS Appl. Mater. Interfaces 2016, 8, 34725.

[201] H.-J. Yun, S.-J. Kang, Y. Xu, S. O. Kim, Y.-H. Kim, Y.-Y. Noh, S.-K. Kwon, Adv. Mater. 2014, 26, 7300.

[202] T. Mukhopadhyay, B. Puttaraju, S. P. Senanayak, A. Sadhanala, R. Friend, H. A. Faber, T. D. Anthopoulos, U. Salzner, A. Meyer S. Patil, ACS Appl. Mater. Interfaces 2016, 8, 25415.

[203] J. Y. Choi, W. Kang, B. Kang, W. Cha, S. K. Son, Y. Yoon, H. Kim Y. Kang, M. J. Ko, H. J. Son, K. Cho, J. H. Cho, B. Kim, ACS Appl. Mater. Interfaces 2015, 7, 6002.

[204] J. Lee, A. R. Han, S. M. Lee, D. Yoo, J. H. Oh, C. Yang, Angew. Chem., Int. Ed. 2015, 54, 4657.

[205] X. Guo, S. R. Puniredd, B. He, T. Marszalek, M. Baumgarten, W. Pisula, K. Mullen, Chem. Mater. 2014, 26, 3595.

[206] J. Yang, H. Wang, J. Chen, J. Huang, Y. Jiang, J. Zhang, L. Shi, Y. Sun, Z. Wei, G. Yu, Y. Guo, S. Wang, Y. Liu, Adv. Mater. 2017, 29 1606162.

[207] M. Gruber, S. H. Jung, S. Schott, D. Venkateshvaran, A. J. Kronemeijer, J. W. Andreasen, C. R. McNeill, W. W. H. Wong, M. Shahid, M. Heeney, J. K. Lee, H. Sirringhaus, Chem. Sci. 2015, 6,6949 .

[208] K.-H. Kim, S. Park, H. Yu, H. Kang, I. Song, J. H. Oh, B. J. Kim, Chem. Mater. 2014, 26, 6963

[209] C. Xiao, G. Zhao, A. Zhang, W. Jiang, R. A. Janssen, W. Li, W. Hu, Z. Wang, Adv. Mater. 2015, 27, 4963.

[210] J. Kim, A. R. Han, J. Hong, G. Kim, J. Lee, T. J. Shin, J. H. Oh, C. Yang, Chem. Mater. 2014, 26, 4933.

[211] H.-J. Yun, H. H. Choi, S.-K. Kwon, Y.-H. Kim, K. Cho, Chem. Mater. 2014, 26, 3928

[212] Y. Gao, X. Zhang, H. Tian, J. Zhang, D. Yan, Y. Geng, F. Wang, Adv. Mater. 2015, 27, 6753

[213] D. Khim, Y. R. Cheon, Y. Xu, W.-T. Park, S.-K. Kwon, Y.-Y. Noh, Y.-H. Kim, Chem. Mater. 2016, 28, 2287.

[214] J.-M. Park, S. K. Park, W. S. Yoon, J. H. Kim, D. W. Kim, T.-L. Choi, S. Y. Park, Macromolecules 2016, 49, 2985.

[215] B. Sun, W. Hong, Z. Yan, H. Aziz, Y. Li, Adv. Mater. 2014, 26, 2636.

[216] P. Li, L. Xu, H. Shen, X. Duan, J. Zhang, Z. Wei, Z. Yi, C. A. Di, S. Wang, ACS Appl. Mater. Interfaces 2016, 8, 8620.

[217] B. Sun, W. Hong, H. Aziz, Y. Li, Polym. Chem. 2015, 6, 938.

[218] J. Lee, A. R. Han, H. Yu, T. J. Shin, C. Yang, J. H. Oh, J. Am. Chem. Soc. 2013, 135, 9540.

[219] L. Janasz, T. Marszalek, W. Zajaczkowski, M. Borkowski, W. Goldeman, A. Kiersnowski, D. Chlebosz, J. Rogowski, P. Blom, J. Ulanski, W. Pisula, J. Mater. Chem. C 2018, 6, 7830.

[220] K. Szendrei, D. Jarzab, Z. Chen, A. Facchetti, M. A. Loi, J. Mater. Chem. 2010, 20, 1317 .

[221] A. Babel, J. D. Wind, S. A. Jenekhe, Adv. Funct. Mater. 2004, 14, 891

[222] S. Nam, H. Han, J. Seo, M. Song, H. Kim, T. D. Anthopoulos, I. McCulloch, D. D. C. Bradley, Y. Kim, Adv. Electron. Mater. 2016 2, 1600264

[223] M. El Gemayel, S. Haar, F. Liscio, A. Schlierf, G. Melinte, S. Milita, O. Ersen, A. Ciesielski, V. Palermo, P. Samori, Adv. Mater. 2014 26, 4814.

[224] L. Janasz, A. Luczak, T. Marszalek, B. G. R. Dupont, J. Jung J. Ulanski, W. Pisula, ACS Appl. Mater. Interfaces 2017, 9, 20696.

[225] Y. Yan, L. B. Huang, Y. Zhou, S. T. Han, L. Zhou, Q. Sun, J. Zhuang, H. Peng, H. Yan, V. A. Roy, ACS Appl. Mater. Interfaces 2015, 7, 23464.

[226] C. Wang, K. Takei, T. Takahashi, A. Javey, Chem. Soc. Rev. 2013, 42, 2592.

[227] S. Park, M. Vosguerichian, Z. Bao, Nanoscale 2013, 5, 1727.
[228] H. Wang, B. Cobb, A. van Breemen, G. Gelinck, Z. Bao, Adv. Mater. 2014, 26, 4588

[229] Z. Wang, S. Liang, Z. Zhang, H. Liu, H. Zhong, L. H. Ye, S. Wang, W. Zhou, J. Liu, Y. Chen, J. Zhang, L. M. Peng, Adv. Mater. 2014 26, 645 .

[230] L. Cai, S. Zhang, J. Miao, Z. Yu, C. Wang, ACS Nano 2016, 10, 11459

[231] H. Chen, Y. Cao, J. Zhang, C. Zhou, Nat. Commun. 2014, 5, 4097.

[232] B. Kim, S. Jang, M. L. Geier, P. L. Prabhumirashi, M. C. Hersam, A. Dodabalapur, Nano Lett. 2014, 14, 3683.

[233] F. Xu, M. Y. Wu, N. S. Safron, S. S. Roy, R. M. Jacobberger, D. J. Bindl, J. H. Seo, T. H. Chang, Z. Ma, M. S. Arnold, Nano Lett. 2014, 14, 682

[234] C. Biswas, Y. H. Lee, Adv. Funct. Mater. 2011, 21, 3806.

[235] S. lijima, Nature 1991, 354, 56.

[236] S. lijima, T. Ichihashi, Nature 1993, 363, 603.

[237] A. D. Franklin, M. Luisier, S. J. Han, G. Tulevski, C. M. Breslin, L. Gignac, M. S. Lundstrom, W. Haensch, Nano Lett. 2012, 12, 758.

[238] A. D. Franklin, Z. Chen, Nat. Nanotechnol. 2010, 5, 858

[239] Z. Liu, J. Zhao, W. Xu, L. Qian, S. Nie, Z. Cui, ACS Appl. Mater Interfaces 2014, 6, 9997.

[240] W. Xu, Z. Liu, J. Zhao, W. Xu, W. Gu, X. Zhang, L. Qian, Z. Cui, Nanoscale 2014, 6, 14891

[241] B. Kim, M. L. Geier, M. C. Hersam, A. Dodabalapur, ACS Appl. Mater. Interfaces 2015, 7, 27654.

[242] P. Chen, Y. Fu, R. Aminirad, C. Wang, J. Zhang, K. Wang, K. Galatsis, C. Zhou, Nano Lett. 2011, 11, 5301.

[243] M. C. Hersam, Nat. Nanotechnol. 2008, 3, 387.

[244] M. S. Arnold, A. A. Green, J. F. Hulvat, S. I. Stupp, M. C. Hersam, Nat. Nanotechnol. 2006, 1,60.

[245] S. Ghosh, S. M. Bachilo, R. B. Weisman, Nat. Nanotechnol. 2010, 5,443 .

[246] X. Tu, S. Manohar, A. Jagota, M. Zheng, Nature 2009, 460, 250.

[247] M. Zheng, A. Jagota, E. D. Semke, B. A. Diner, R. S. McLean, S. R. Lustig, R. E. Richardson, N. G. Tassi, Nat. Mater. 2003, 2, 338

[248] H. Liu, D. Nishide, T. Tanaka, H. Kataura, Nat. Commun. 2011, 2, 309.

[249] R. Krupke, F. Hennrich, H. v. Löhneysen, M. M. Kappes, Science 2003, 301, 344 .

[250] T. Tanaka, H. Jin, Y. Miyata, H. Kataura, Appl. Phys. Express 2008, 1, 114001.

[251] S. K. Samanta, M. Fritsch, U. Scherf, W. Gomulya, S. Z. Bisri, M. A. Loi, Acc. Chem. Res. 2014, 47, 2446.

[252] A. Nish, J. Y. Hwang, J. Doig, R. J. Nicholas, Nat. Nanotechnol. 2007, 2, 640 .

[253] S. Z. Bisri, J. Gao, V. Derenskyi, W. Gomulya, I. lezhokin, P. Gordiichuk, A. Herrmann, M. A. Loi, Adv. Mater. 2012, 24, 6147.

[254] H. Ozawa, N. Ide, T. Fujigaya, Y. Niidome, N. Nakashima, Chem. Lett. 2011, 40, 239.

[255] V. Derenskyi, W. Gomulya, J. M. Rios, M. Fritsch, N. Frohlich, S. Jung, S. Allard, S. Z. Bisri, P. Gordiichuk, A. Herrmann, U. Scherf, M. A. Loi, Adv. Mater. 2014, 26, 5969.

[256] S. H. Lee, D. Khim, Y. Xu, J. Kim, W. T. Park, D. Y. Kim, Y. Y. Noh, Sci. Rep. 2015, 5, 10407

[257] J. Gao, M. Kwak, J. Wildeman, A. Herrmann, M. A. Loi, Carbon 2011, 49, 333

[258] H. W. Lee, Y. Yoon, S. Park, J. H. Oh, S. Hong, L. S. Liyanage, H. Wang, S. Morishita, N. Patil, Y. J. Park, J. J. Park, A. Spakowitz, G. Galli, F. Gygi, P. H. Wong, J. B. Tok, J. M. Kim, Z. Bao, Nat. Commun. 2011, 2, 541.

[259] N. Izard, S. Kazaoui, K. Hata, T. Okazaki, T. Saito, S. lijima, N. Minami, Appl. Phys. Lett. 2008, 92, 243112.

[260] H. Wang, G. I. Koleilat, P. Liu, G. Jiménez-Osés, Y.-C. Lai, M. Vosgueritchian, Y. Fang, S. Park, K. N. Houk, Z. Bao, ACS Nano $2014,8,2609$ 
[261] V. Derenskyi, W. Gomulya, J. Gao, S. Z. Bisri, M. Pasini, Y.-L. Loo, M. A. Loi, Appl. Phys. Lett. 2018, 112, 072106.

[262] J. M. Salazar-Rios, W. Gomulya, V. Derenskyi, J. Yang, S. Z. Bisri, Z. Chen, A. Facchetti, M. A. Loi, Adv. Electron. Mater. 2015, 1, 1500074.

[263] W. Gomulya, G. D. Costanzo, E. J. de Carvalho, S. Z. Bisri, V. Derenskyi, M. Fritsch, N. Frohlich, S. Allard, P. Gordiichuk, A. Herrmann, S. J. Marrink, M. C. dos Santos, U. Scherf, M. A. Loi, Adv. Mater. 2013, 25, 2948.

[264] N. Rouhi, D. Jain, P. J. Burke, ACS Nano 2011, 5, 8471.

[265] Z. Liu, L. Jiao, Y. Yao, X. Xian, J. Zhang, Adv. Mater. 2010, 22, 2285.

[266] S. Shekhar, P. Stokes, S. I. Khondaker, ACS Nano 2011, 5, 1739.

[267] M. D. Lay, J. P. Novak, E. S. Snow, Nano Lett. 2004, 4, 603.

[268] Q. Cao, S. J. Han, G. S. Tulevski, Y. Zhu, D. D. Lu, W. Haensch, Nat. Nanotechnol. 2013, 8, 180.

[269] H. Park, A. Afzali, S. J. Han, G. S. Tulevski, A. D. Franklin, J. Tersoff, J. B. Hannon, W. Haensch, Nat. Nanotechnol. 2012, 7, 787.

[270] V. Derenskyi, W. Gomulya, W. Talsma, J. M. Salazar-Rios, M. Fritsch, P. Nirmalraj, H. Riel, S. Allard, U. Scherf, M. A. Loi, Adv. Mater. 2017, 29, 1606757.

[271] M. Kwak, J. Gao, D. K. Prusty, A. J. Musser, V. A. Markov, N. Tombros, M. C. Stuart, W. R. Browne, E. J. Boekema, G. ten Brinke, H. T. Jonkman, B. J. van Wees, M. A. Loi, A. Herrmann, Angew. Chem., Int. Ed. 2011, 50, 3206.

[272] M. Engel, J. P. Small, M. Steiner, M. Freitag, A. A. Green, M. C. Hersam, P. Avouris, ACS Nano 2008, 2, 2445.

[273] J. Zhang, C. Wang, Y. Fu, Y. Che, C. Zhou, ACS Nano 2011, 5, 3284.

[274] L. Ding, Z. Zhang, S. Liang, T. Pei, S. Wang, Y. Li, W. Zhou, J. Liu, L. M. Peng, Nat. Commun. 2012, 3, 677.

[275] L. Suriyasena Liyanage, X. Xu, G. Pitner, Z. Bao, H. S. Wong, Nano Lett. 2014, 14, 1884.

[276] K. Raj, X. Pan, Q. Zhang, M. B. Chan-Park, G. Pingqi, Chem. Phys. Lett. 2009, 470, 95.

[277] Q. Xu, J. Zhao, V. Pecunia, W. Xu, C. Zhou, J. Dou, W. Gu, J. Lin, L. Mo, Y. Zhao, Z. Cui, ACS Appl. Mater. Interfaces 2017, 9, 12750.

[278] M. Ha, J. W. Seo, P. L. Prabhumirashi, W. Zhang, M. L. Geier, M. J. Renn, C. H. Kim, M. C. Hersam, C. D. Frisbie, Nano Lett. 2013, 13, 954.

[279] M. Ha, Y. Xia, A. A. Green, W. Zhang, M. J. Renn, C. H. Kim, M. C. Hersam, C. D. Frisbie, ACS Nano 2010, 4, 4388.

[280] B. Kim, K. Liang, M. L. Geier, M. C. Hersam, A. Dodabalapur, Appl. Phys. Lett. 2016, 109, 023515.

[281] M. Yu, H. Wan, L. Cai, J. Miao, S. Zhang, C. Wang, ACS Nano 2018, 12, 11572.

[282] K. S. Novoselov, A. K. Geim, S. V. Morozov, D. Jiang, Y. Zhang, S. V. Dubonos, I. V. Grigorieva, A. A. Firsov, Science 2004, 306, 666.

[283] P. R. Pudasaini, A. Oyedele, C. Zhang, M. G. Stanford, N. Cross, A. T. Wong, A. N. Hoffman, K. Xiao, G. Duscher, D. G. Mandrus, T. Z. Ward, P. D. Rack, Nano Res. 2018, 11, 722.

[284] D. Qu, X. Liu, M. Huang, C. Lee, F. Ahmed, H. Kim, R. S. Ruoff, J. Hone, W. J. Yoo, Adv. Mater. 2017, 29, 1606433.

[285] Y. Cai, J. Shen, G. Ge, Y. Zhang, W. Jin, W. Huang, J. Shao, J. Yang, X. Dong, ACS Nano 2018, 12, 56.

[286] H. Fang, S. Chuang, T. C. Chang, K. Takei, T. Takahashi, A. Javey, Nano Lett. 2012, 12, 3788.

[287] S. Das, J. Appenzeller, Appl. Phys. Lett. 2013, 103, 103501.

[288] B. Hunt, J. D. Sanchez-Yamagishi, A. F. Young, M. Yankowitz, B. J. LeRoy, K. Watanabe, T. Taniguchi, P. Moon, M. Koshino, P. Jarillo-Herrero, R. C. Ashoori, Science 2013, 340, 1427.

[289] W. T. Hsu, L. S. Lu, P. H. Wu, M. H. Lee, P. J. Chen, P. Y. Wu, Y. C. Chou, H. T. Jeng, L. J. Li, M. W. Chu, W. H. Chang, Nat. Commun. 2018, 9, 1356.

[290] Y. Cao, V. Fatemi, S. Fang, K. Watanabe, T. Taniguchi, E. Kaxiras, P. Jarillo-Herrero, Nature 2018, 556, 43.
[291] G. Chen, L. Jiang, S. Wu, B. Lyu, H. Li, B. L. Chittari, K. Watanabe, T. Taniguchi, Z. Shi, J. Jung, Y. Zhang, F. Wang, Nat. Phys. 2019, 15, 237.

[292] M. Buscema, D. J. Groenendijk, S. I. Blanter, G. A. Steele, H. S. van der Zant, A. Castellanos-Gomez, Nano Lett. 2014, 14, 3347.

[293] W. Liu, J. Kang, D. Sarkar, Y. Khatami, D. Jena, K. Banerjee, Nano Lett. 2013, 13, 1983.

[294] X. Zhang, Z. Shao, X. Zhang, Y. He, J. Jie, Adv. Mater. 2016, 28, 10409.

[295] C. Zhou, Y. Zhao, S. Raju, Y. Wang, Z. Lin, M. Chan, Y. Chai, Adv. Funct. Mater. 2016, 26, 4223.

[296] Z. Wang, Q. Li, Y. Chen, B. Cui, Y. Li, F. Besenbacher, M. Dong, NPG Asia Mater. 2018, 10, 703.

[297] K. F. Mak, C. Lee, J. Hone, J. Shan, T. F. Heinz, Phys. Rev. Lett. 2010, 105, 136805.

[298] H. Liu, P. D. Ye, IEEE Electron Device Lett. 2012, 33, 546.

[299] B. Radisavljevic, A. Radenovic, J. Brivio, V. Giacometti, A. Kis, Nat. Nanotechnol. 2011, 6, 147.

[300] Y. J. Zhang, J. T. Ye, Y. Yomogida, T. Takenobu, Y. Iwasa, Nano Lett. 2013, 13, 3023.

[301] W. Bao, X. Cai, D. Kim, K. Sridhara, M. S. Fuhrer, Appl. Phys. Lett. 2013, 102, 042104.

[302] Y. Zhang, J. Ye, Y. Matsuhashi, Y. Iwasa, Nano Lett. 2012, 12, 1136

[303] J. T. Ye, Y. J. Zhang, R. Akashi, M. S. Bahramy, R. Arita, Y. Iwasa, Science 2012, 338, 1193

[304] M. M. Perera, M.-W. Lin, H.-J. Chuang, B. P. Chamlagain, C. Wang, X. Tan, M. M.-C. Cheng, D. Tománek, Z. Zhou, ACS Nano 2013, 7, 4449.

[305] E. Ponomarev, I. Gutierrez-Lezama, N. Ubrig, A. F. Morpurgo, Nano Lett. 2015, 15, 8289.

[306] D. Costanzo, S. Jo, H. Berger, A. F. Morpurgo, Nat. Nanotechnol. 2016, 11, 339

[307] K. Jin, W. Hu, B. Zhu, D. Kim, J. Yuan, Y. Sun, T. Xiang, M. S. Fuhrer, I. Takeuchi, R. L. Greene, Sci. Rep. 2016, 6, 26642.

[308] Z. J. Li, B. F. Gao, J. L. Zhao, X. M. Xie, M. H. Jiang, Supercond. Sci. Technol. 2014, 27, 015004

[309] C. Jang, S. Adam, J. H. Chen, E. D. Williams, S. Das Sarma, M. S. Fuhrer, Phys. Rev. Lett. 2008, 101, 146805.

[310] J. H. Choi, H. K. Jang, J. E. Jin, J. M. Shin, D. H. Kim, G. T. Kim, Appl. Phys. Lett. 2016, 109, 183102.

[311] Q. H. Wang, K. Kalantar-Zadeh, A. Kis, J. N. Coleman, M. S. Strano, Nat. Nanotechnol. 2012, 7, 699

[312] Y. F. Lin, Y. Xu, S. T. Wang, S. L. Li, M. Yamamoto A. Aparecido-Ferreira, W. Li, H. Sun, S. Nakaharai, W. B. Jian, K. Ueno, K. Tsukagoshi, Adv. Mater. 2014, 26, 3263

[313] Y. Ma, Y. Dai, M. Guo, C. Niu, J. Lu, B. Huang, Phys. Chem. Chem. Phys. 2011, 13, 15546.

[314] S. Nakaharai, M. Yamamoto, K. Ueno, Y.-F. Lin, S.-L. Li, K. Tsukagoshi, ACS Nano 2015, 9, 5976.

[315] S. Fathipour, N. Ma, W. S. Hwang, V. Protasenko, S. Vishwanath, H. G. Xing, H. Xu, D. Jena, J. Appenzeller, A. Seabaugh, Appl. Phys. Lett. 2014, 105, 192101.

[316] W. Luo, M. Zhu, G. Peng, X. Zheng, F. Miao, S. Bai, X.-A. Zhang, S. Qin, Adv. Funct. Mater. 2018, 28, 1704539.

[317] Y. Li, K. Zhang, F. Wang, Y. Feng, Y. Li, Y. Han, D. Tang, B. Zhang, ACS Appl. Mater. Interfaces 2017, 9, 36009

[318] N. R. Pradhan, Z. Lu, D. Rhodes, D. Smirnov, E. Manousakis, L. Balicas, Adv. Electron. Mater. 2015, 1, 1500215.

[319] M. W. Chen, D. Ovchinnikov, S. Lazar, M. Pizzochero, M. B. Whitwick, A. Surrente, M. Baranowski, O. L. Sanchez, P. Gillet, P. Plochocka, O. V. Yazyev, A. Kis, ACS Nano 2017, 11, 6355.

[320] D. N. Ortiz, I. Ramos, N. J. Pinto, M.-Q. Zhao, V. Kumar, A. C. Johnson, AIP Adv. 2018, 8, 035014.

[321] S. Jo, N. Ubrig, H. Berger, A. B. Kuzmenko, A. F. Morpurgo, Nano Lett. 2014, 14, 2019. 
[322] W. S. Hwang, M. Remskar, R. Yan, V. Protasenko, K. Tahy, S. D. Chae, P. Zhao, A. Konar, H. Xing, A. Seabaugh, D. Jena, Appl. Phys. Lett. 2012, 101, 013107.

[323] D. Braga, I. Gutierrez Lezama, H. Berger, A. F. Morpurgo, Nano Lett. 2012, 12, 5218

[324] A. Prakash, J. Appenzeller, ACS Nano 2017, 11, 1626.

[325] M. Yoshida, T. lizuka, Y. Saito, M. Onga, R. Suzuki, Y. Zhang, Y. Iwasa, S. Shimizu, Nano Lett. 2016, 16, 2061.

[326] D. Li, X. Wang, Y. Chen, S. Zhu, F. Gong, G. Wu, C. Meng, L. Liu, L. Wang, T. Lin, S. Sun, H. Shen, X. Wang, W. Hu, J. Wang, J. Sun, X. Meng, J. Chu, Nanotechnology 2018, 29, 105202.

[327] M. Nakano, Y. Wang, Y. Kashiwabara, H. Matsuoka, Y. Iwasa, Nano Lett. 2017, 17, 5595 .

[328] E. Zhang, R. Chen, C. Huang, J. Yu, K. Zhang, W. Wang, S. Liu, J. Ling, X. Wan, H. Z. Lu, F. Xiu, Nano Lett. 2017, 17, 878

[329] Y. Zhao, J. Qiao, Z. Yu, P. Yu, K. Xu, S. P. Lau, W. Zhou, Z. Liu, X. Wang, W. Ji, Y. Chai, Adv. Mater. 2017, 29, 1604230.

[330] S. T. Han, L. Hu, X. Wang, Y. Zhou, Y. J. Zeng, S. Ruan, C. Pan, Z. Peng, Adv. Sci. 2017, 4, 1600435

[331] J. Kang, J. D. Wood, S. A. Wells, J. H. Lee, X. L. Liu, K. S. Chen, M. C. Hersam, ACS Nano 2015, 9, 3596.

[332] W. Zhu, M. N. Yogeesh, S. Yang, S. H. Aldave, J. S. Kim, S. Sonde, L. Tao, N. Lu, D. Akinwande, Nano Lett. 2015, 15, 1883

[333] S. Das, M. Demarteau, A. Roelofs, ACS Nano 2014, 8, 11730

[334] Y. Du, H. Liu, Y. Deng, P. D. Ye, ACS Nano 2014, 8, 10035.

[335] H. Park, J. Kim, Phys. Chem. Chem. Phys. 2018, 20, 22439

[336] Y. Saito, Y. Iwasa, ACS Nano 2015, 9, 3192.

[337] D. Xiang, C. Han, J. Wu, S. Zhong, Y. Liu, J. Lin, X. A. Zhang, W. Ping Hu, B. Ozyilmaz, A. H. Neto, A. T. Wee, W. Chen, Nat. Commun. 2015, 6, 6485.

[338] D. Yue, D. Lee, Y. D. Jang, M. S. Choi, H. J. Nam, D. Y. Jung, W. J. Yoo, Nanoscale 2016, 8, 12773.

[339] Q. Gui, X. Zhu, L. Liu, Z. Y. Jia, Y. H. Song, S. C. Li, P. K. Chu, X. Wu, ACS Appl. Mater. Interfaces 2017, 9, 39804.

[340] A. K. Geim, I. V. Grigorieva, Nature 2013, 499, 419.

[341] L. Britnell, R. V. Gorbachev, R. Jalil, B. D. Belle, F. Schedin, A. Mishchenko, T. Georgiou, M. I. Katsnelson, L. Eaves, S. V. Morozov, N. M. R. Peres, J. Leist, A. K. Geim, K. S. Novoselov, L. A. Ponomarenko, Science 2012, 335, 947.

[342] L. Britnell, R. M. Ribeiro, A. Eckmann, R. Jalil, B. D. Belle, A. Mishchenko, Y.-J. Kim, R. V. Gorbachev, T. Georgiou, S. V. Morozov, A. N. Grigorenko, A. K. Geim, C. Casiraghi, A. H. C. Neto, K. S. Novoselov, Science 2013, 340, 1311.

[343] B. Hunt, J. Sanchez-Yamagishi, A. Young, M. Yankowitz, B. J. LeRoy, K. Watanabe, T. Taniguchi, P. Moon, M. Koshino, P. Jarillo-Herrero, Science 2013, 340, 1427.

[344] N. Huo, J. Kang, Z. Wei, S.-S. Li, J. Li, S.-H. Wei, Adv. Funct. Mater. 2014, 24, 7025

[345] N. Huo, J. Yang, L. Huang, Z. Wei, S. S. Li, S. H. Wei, J. Li, Small 2015, 11, 5430

[346] C. Wang, S. Yang, W. Xiong, C. Xia, H. Cai, B. Chen, X. Wang, X. Zhang, Z. Wei, S. Tongay, J. Li, Q. Liu, Phys. Chem. Chem. Phys. 2016, 18, 27750.

[347] S. Yang, C. Wang, C. Ataca, Y. Li, H. Chen, H. Cai, A. Suslu, J. C. Grossman, C. Jiang, Q. Liu, S. Tongay, ACS Appl. Mater. Interfaces 2016, 8, 2533.

[348] X. Li, M.-W. Lin, J. Lin, B. Huang, A. A. Puretzky, C. Ma, K. Wang, W. Zhou, S. T. Pantelides, M. Chi, I. Kravchenko, J. Fowlkes, C. M. Rouleau, D. B. Geohegan, K. Xiao, Sci. Adv. 2016, 2, e1501882.

[349] J. Dong, F. Liu, F. Wang, J. Wang, M. Li, Y. Wen, L. Wang, G. Wang, J. He, C. Jiang, Nanoscale 2017, 9, 7519.

[350] X. He, W. Chow, F. Liu, B. Tay, Z. Liu, Small 2017, 13, 1602558.

[351] J. Yan, Y. Hao, Y. Cui, J. Zhang, Y. Zou, W. Zhang, G. Yu, J. Zheng, W. Xu, D. Zhu, J. Mater. Chem. C 2018, 6, 12976.
[352] D. He, Y. Zhang, Q. Wu, R. Xu, H. Nan, J. Liu, J. Yao, Z. Wang, S. Yuan, Y. Li, Y. Shi, J. Wang, Z. Ni, L. He, F. Miao, F. Song, H. Xu, K. Watanabe, T. Taniguchi, J. B. Xu, X. Wang, Nat. Commun. 2014 $5,5162$.

[353] B. Park, K. Kim, J. Park, H. Lim, P. T. Lanh, A. R. Jang, C. Hyun, C. W. Myung, S. Park, J. W. Kim, K. S. Kim, H. S. Shin, G. Lee, S. H. Kim, C. E. Park, J. K. Kim, ACS Appl. Mater. Interfaces 2017, 9, 27839.

[354] I. Lee, S. Rathi, D. Lim, L. Li, J. Park, Y. Lee, K. S. Yi, K. P. Dhakal, J. Kim, C. Lee, G.-H. Lee, Y. D. Kim, J. Hone, S. J. Yun, D.-H. Youn, G.-H. Kim, Adv. Mater. 2016, 28, 9519.

[355] X. Ding, L. Chen, Y. Honsho, X. Feng, O. Saengsawang, J. Guo, A. Saeki, S. Seki, S. Irle, S. Nagase, V. Parasuk, D. Jiang, J. Am. Chem. Soc. 2011, 133, 14510.

[356] M. Calik, F. Auras, L. M. Salonen, K. Bader, I. Grill, M. Handloser, D. D. Medina, M. Dogru, F. Lobermann, D. Trauner, A. Hartschuh, T. Bein, J. Am. Chem. Soc. 2014, 136, 17802.

[357] W. Liu, X. Luo, Y. Bao, Y. P. Liu, G. H. Ning, I. Abdelwahab, L. Li, C. T. Nai, Z. G. Hu, D. Zhao, B. Liu, S. Y. Quek, K. P. Loh, Nat. Chem. 2017, 9, 563.

[358] X. Zhang, X. Ren, B. Liu, R. Munir, X. Zhu, D. Yang, J. Li, Y. Liu, D.-M. Smilgies, R. Li, Z. Yang, T. Niu, X. Wang, A. Amassian, K. Zhao, S. Liu, Energy Environ. Sci. 2017, 10, 2095.

[359] X. Li, J. Yang, Z. Song, R. Chen, L. Ma, H. Li, J. Jia, J. Meng, X. Li, M. Yi, X. Sun, ACS Appl. Energy Mater. 2018, 1, 4467.

[360] B. Sun, C.-H. Zhu, Y. Liu, C. Wang, L.-J. Wan, D. Wang, Chem. Mater. 2017, 29, 4367.

[361] X. Huang, P. Sheng, Z. Tu, F. Zhang, J. Wang, H. Geng, Y. Zou, C.-a. Di, Y. Yi, Y. Sun, W. Xu, D. Zhu, Nat. Commun. 2015, 6, 7408.

[362] I. van Weperen, S. R. Plissard, E. P. Bakkers, S. M. Frolov, L. P. Kouwenhoven, Nano Lett. 2013, 13, 387.

[363] V. S. Pribiag, S. Nadj-Perge, S. M. Frolov, J. W. van den Berg, I. van Weperen, S. R. Plissard, E. P. Bakkers, L. P. Kouwenhoven, Nat. Nanotechnol. 2013, 8, 170

[364] Y. Zhao, J. T. Smith, J. Appenzeller, C. Yang, Nano Lett. 2011, 11 1406.

[365] T. Rieger, P. Zellekens, N. Demarina, A. A. Hassan, F. J. Hackemuller, H. Luth, U. Pietsch, T. Schapers, D. Grutzmacher, M. I. Lepsa, Nanoscale 2017, 9, 18392.

[366] R. Cox, G. T. Olson, M. Pfau, N. Eshaghi, K. Barcus, D. Ramirez, R. Fernando, S. Zhang, ACS Appl. Mater. Interfaces 2017, 9, 16397.

[367] S. Z. Bisri, C. Piliego, M. Yarema, W. Heiss, M. A. Loi, Adv. Mater. 2013, 25, 4309.

[368] S. K. Garlapati, M. Divya, B. Breitung, R. Kruk, H. Hahn, S. Dasgupta, Adv. Mater. 2018, 30, 1707600.

[369] M. S. Kang, J. Lee, D. J. Norris, C. D. Frisbie, Nano Lett. 2009, 9, 3848.

[370] Y. Zhou, S. T. Han, Z. X. Xu, V. A. Roy, Adv. Mater. 2012, 24, 1247.

[371] M. I. Nugraha, H. Matsui, S. Z. Bisri, M. Sytnyk, W. Heiss, M. A. Loi, J. Takeya, APL Mater. 2016, 4, 116105.

[372] M. I. Nugraha, H. Matsui, S. Watanabe, T. Kubo, R. Häusermann, S. Z. Bisri, M. Sytnyk, W. Heiss, M. A. Loi, J. Takeya, Adv. Electron. Mater. 2017, 3, 1600360.

[373] A. G. Shulga, S. Kahmann, D. N. Dirin, A. Graf, J. Zaumseil, M. V. Kovalenko, M. A. Loi, ACS Nano 2018, 12, 12805.

[374] L. Han, D. M. Balazs, A. G. Shulga, M. Abdu-Aguye, W. Ma, M. A. Loi, Adv. Electron. Mater. 2018, 4, 1700580.

[375] M. I. Nugraha, R. Häusermann, S. Z. Bisri, H. Matsui, M. Sytnyk, W. Heiss, J. Takeya, M. A. Loi, Adv. Mater. 2015, 27, 2107.

[376] A. G. Shulga, L. Piveteau, S. Z. Bisri, M. V. Kovalenko, M. A. Loi, Adv. Electron. Mater. 2016, 2, 1500467.

[377] S. Z. Bisri, E. Degoli, N. Spallanzani, G. Krishnan, B. J. Kooi, C. Ghica, M. Yarema, W. Heiss, O. Pulci, S. Ossicini, M. A. Loi, Adv. Mater. 2014, 26, 5639. 
[378] H. Kim, A. P. Tiwari, E. Hwang, Y. Cho, H. Hwang, S. Bak, Y. Hong, H. Lee, Adv. Sci. 2018, 5, 1800068.

[379] J. Yoo, J. Pyo, J. H. Je, Nanoscale 2014, 6, 3557.

[380] W. Liu, J. S. Lee, D. V. Talapin, J. Am. Chem. Soc. 2013, 135, 1349.

[381] J. Burschka, N. Pellet, S. J. Moon, R. Humphry-Baker, P. Gao, M. K. Nazeeruddin, M. Gratzel, Nature 2013, 499, 316.

[382] M. M. Lee, J. Teuscher, T. Miyasaka, T. N. Murakami, H. J. Snaith, Science 2012, 338, 643.

[383] L. Gu, M. M. Tavakoli, D. Zhang, Q. Zhang, A. Waleed, Y. Xiao, K.-H. Tsui, Y. Lin, L. Liao, J. Wang, Z. Fan, Adv. Mater. 2016, 28, 9713.

[384] Y.-M. You, W.-Q. Liao, D. Zhao, H.-Y. Ye, Y. Zhang, Q. Zhou, X. Niu, J. Wang, P.-F. Li, D.-W. Fu, Z. Wang, S. Gao, K. Yang, J.-M. Liu, J. Li, Y. Yan, R.-G. Xiong, Science 2017, 357, 306.

[385] C. Gu, J. S. Lee, ACS Nano 2016, 10, 5413.

[386] J. Choi, J. S. Han, K. Hong, S. Y. Kim, H. W. Jang, Adv. Mater. 2018, 30, 1704002.

[387] T. Zhao, D. Wang, Z. Shuai, Synth. Met. 2017, 225, 108.

[388] C. R. Kagan, D. B. Mitzi, C. D. Dimitrakopoulos, Science 1999, 286, 945.

[389] L. Tang, Y. Peng, Z. Zhou, Y. Wu, J. Xu, J. Li, Y. Du, L. Huang, H. Cai, J. Ni, J. Zhang, Appl. Phys. A 2018, 124, 624.

[390] Y. Mei, C. Zhang, Z. V. Vardeny, O. D. Jurchescu, MRS Commun. 2015, 5, 297.

[391] A. R. Yusoff, H. P. Kim, X. Li, J. Kim, J. Jang, M. K. Nazeeruddin, Adv. Mater. 2017, 29, 1602940.

[392] G. E. Eperon, S. D. Stranks, C. Menelaou, M. B. Johnston, L. M. Herz, H. J. Snaith, Energy Environ. Sci. 2014, 7, 982.

[393] Q. Han, S.-H. Bae, P. Sun, Y.-T. Hsieh, Y. M. Yang, Y. S. Rim, H. Zhao, Q. Chen, W. Shi, G. Li, Y. Yang, Adv. Mater. 2016, 28, 2253.

[394] H. Luo, L. Liang, H. Cao, M. Dai, Y. Lu, M. Wang, ACS Appl. Mater. Interfaces 2015, 7, 17023.

[395] D. Y. Jeon, S. Pregl, S. J. Park, L. Baraban, G. Cuniberti, T. Mikolajick, W. M. Weber, Nano Lett. 2015, 15, 4578.

[396] S. Sun, Y. Li, L. Lan, P. Xiao, Z. Chen, Z. Lin, J. Chen, J. Peng, Y. Cao, Org. Electron. 2017, 43, 162.

[397] M. Li, J. Wang, X. Cai, F. Liu, X. Li, L. Wang, L. Liao, C. Jiang, Adv. Electron. Mater. 2018, 4, 1800211.

[398] J. Smith, A. Bashir, G. Adamopoulos, J. E. Anthony, D. D. Bradley, M. Heeney, I. McCulloch, T. D. Anthopoulos, Adv. Mater. 2010, 22, 3598.

[399] B. Kim, S. Jang, M. L. Geier, P. L. Prabhumirashi, M. C. Hersam, A. Dodabalapur, Appl. Phys. Lett. 2014, 104, 062101.

[400] S. Sun, L. Lan, Y. Li, H. Ning, R. Yao, L. Wang, J. Peng, RSC Adv. 2017, 7, 5966.

[401] H. Nakanotani, M. Yahiro, C. Adachi, K. Yano, Appl. Phys. Lett. 2007, 90, 262104

[402] Y. Zhou, S.-T. Han, L. Zhou, Y. Yan, L.-B. Huang, J. Huang, V. A. L. Roy, J. Mater. Chem. C 2013, 1, 7073.

[403] C. Yang, Y. Kwack, S. H. Kim, T. K. An, K. Hong, S. Nam, M. Park, W.-S. Choi, C. E. Park, Org. Electron. 2011, 12, 411.

[404] A. A. Green, M. C. Hersam, Adv. Mater. 2011, 23, 2185.

[405] K. Nomura, H. Ohta, A. Takagi, T. Kamiya, M. Hirano, H. Hosono, Nature 2004, 432, 488.

[406] M. H. Boratto, L. V. A. Scalvi, L. V. Goncharova, G. Fanchini, J. Mater. Sci.: Mater. Electron. 2018, 29, 20010.

[407] X. J. She, D. Gustafsson, H. Sirringhaus, Adv. Mater. 2017, 29, 1604769.

[408] D. Li, M. Chen, Q. Zong, Z. Zhang, Nano Lett. 2017, 17, 6353.

[409] Y. S. Park, J. S. Lee, Adv. Mater. 2015, 27, 706.

[410] D. Lee, Y. Choi, E. Hwang, M. S. Kang, S. Lee, J. H. Cho, Nanoscale 2016, 8, 9107.

[411] C. C. Shih, W. Y. Lee, W. C. Chen, Mater. Horiz. 2016, 3, 294.

[412] R. H. Kim, H. J. Kim, I. Bae, S. K. Hwang, D. B. Velusamy, S. M. Cho, K. Takaishi, T. Muto, D. Hashizume, M. Uchiyama,
P. Andre, F. Mathevet, B. Heinrich, T. Aoyama, D. E. Kim, H. Lee, J. C. Ribierre, C. Park, Nat. Commun. 2014, 5, 3583.

[413] E. Zhang, W. Wang, C. Zhang, Y. Jin, G. Zhu, Q. Sun, D. W. Zhang, P. Zhou, F. Xiu, ACS Nano 2015, 9, 612.

[414] J. Han, W. Wang, J. Ying, W. Xie, Appl. Phys. Lett. 2014, 104, 013302.

[415] Y. Che, Y. Zhang, X. Cao, X. Song, M. Cao, H. Dai, J. Yang, G. Zhang, J. Yao, Appl. Phys. Lett. 2016, 109, 013106.

[416] W. Kang, M. Jung, W. Cha, S. Jang, Y. Yoon, H. Kim, H. J. Son, D. K. Lee, B. Kim, J. H. Cho, ACS Appl. Mater. Interfaces 2014, 6, 6589.

[417] Y. Zhou, S. T. Han, P. Sonar, V. A. Roy, Sci. Rep. 2013, 3, 2319.

[418] Z. Chen, Y. Zhang, H. Zhang, Y. Yu, X. Song, H. Zhang, M. Cao, Y. Che, L. Jin, Y. Li, Q. Li, H. Dai, J. Yang, J. Yao, Appl. Phys. Lett. 2018, 112, 212101.

[419] H. Zhang, Y. Zhang, Y. Yu, X. Song, H. Zhang, M. Cao, Y. Che, H. Dai, J. Yang, J. Yao, ACS Photonics 2017, 4, 2220.

[420] K. J. Baeg, M. G. Kim, C. K. Song, X. Yu, A. Facchetti, T. J. Marks, Adv. Mater. 2014, 26, 7170 .

[421] Y.-H. Chou, H.-C. Chang, C.-L. Liu, W.-C. Chen, Polym. Chem. 2015, 6, 341

[422] S.-W. Cheng, Y.-H. Chang Chien, T.-Y. Huang, C.-L. Liu, G.-S. Liou, Polymer 2018, 148, 382.

[423] W. Wang, K. L. Kim, S. M. Cho, J. H. Lee, C. Park, ACS Appl. Mater. Interfaces 2016, 8, 33863.

[424] G. Wang, X. Liu, W. Wang, IEEE Electron Device Lett. 2018, 39, 111.

[425] M. Kang, D. Khim, J. Kim, H. J. Lee, J. Y. Jo, K.-J. Baeg, D.-Y. Kim, Org. Electron. 2018, 58, 12.

[426] W. Wang, S. K. Hwang, K. L. Kim, J. H. Lee, S. M. Cho, C. Park, ACS Appl. Mater. Interfaces 2015, 7, 10957.

[427] Y. Che, Y. Zhang, X. Cao, X. Song, M. Cao, H. Dai, J. Yang, G. Zhang, J. Yao, J. Mater. Chem. C 2016, 4, 1420.

[428] S. T. Han, Y. Zhou, P. Sonar, H. Wei, L. Zhou, Y. Yan, C. S. Lee, V. A. Roy, ACS Appl. Mater. Interfaces 2015, 7, 1699.

[429] M. Yi, M. Xie, Y. Shao, W. Li, H. Ling, L. Xie, T. Yang, Q. Fan, J. Zhu, W. Huang, J. Mater. Chem. C 2015, 3, 5220.

[430] C. Mead, Proc. IEEE 1990, 78, 1629.

[431] Y. van de Burgt, A. Melianas, S. T. Keene, G. Malliaras, A. Salleo, Nat. Electron. 2018, 1, 386.

[432] S. Fusi, M. Annunziato, D. Badoni, A. Salamon, D. J. Amit, Neural Comput. 2000, 12, 2227.

[433] R. Yang, H.-M. Huang, Q.-H. Hong, X.-B. Yin, Z.-H. Tan, T. Shi, Y.-X. Zhou, X.-S. Miao, X.-P. Wang, S.-B. Mi, C.-L. Jia, X. Guo, Adv. Funct. Mater. 2018, 28, 1704455.

[434] X. Yan, L. Zhang, H. Chen, X. Li, J. Wang, Q. Liu, C. Lu, J. Chen, H. Wu, P. Zhou, Adv. Funct. Mater. 2018, 28, 1803728.

[435] H. Tian, W. Mi, H. Zhao, M. A. Mohammad, Y. Yang, P. W. Chiu, T. L. Ren, Nanoscale 2017, 9, 9275.

[436] A. Chanthbouala, V. Garcia, R. O. Cherifi, K. Bouzehouane, S. Fusil, X. Moya, S. Xavier, H. Yamada, C. Deranlot, N. D. Mathur, M. Bibes, A. Barthelemy, J. Grollier, Nat. Mater. 2012, 11, 860.

[437] A. J. Arnold, A. Razavieh, J. R. Nasr, D. S. Schulman, C. M. Eichfeld, S. Das, ACS Nano 2017, 11, 3110 .

[438] H. Tian, X. Cao, Y. Xie, X. Yan, A. Kostelec, D. DiMarzio, C. Chang, L. D. Zhao, W. Wu, J. Tice, J. J. Cha, J. Guo, H. Wang, ACS Nano 2017, 11, 7156.

[439] Y.-N. Zhong, T. Wang, X. Gao, J.-L. Xu, S.-D. Wang, Adv. Funct. Mater. 2018, 28, 1800854.

[440] B. Li, Y. Liu, C. Wan, Z. Liu, M. Wang, D. Qi, J. Yu, P. Cai, M. Xiao, Y. Zeng, X. Chen, Adv. Mater. 2018, 30, 1706395.

[441] P. Yao, H. Wu, B. Gao, S. B. Eryilmaz, X. Huang, W. Zhang, Q. Zhang, N. Deng, L. Shi, H. P. Wong, H. Qian, Nat. Commun. 2017, 8, 15199.

[442] Z. Wang, S. Joshi, S. E. Savel'ev, H. Jiang, R. Midya, P. Lin, M. Hu, N. Ge, J. P. Strachan, Z. Li, Q. Wu, M. Barnell, G. L. Li, H. L. Xin, R. S. Williams, Q. Xia, J. J. Yang, Nat. Mater. 2017, 16, 101. 
[443] L. Q. Zhu, C. J. Wan, L. Q. Guo, Y. Shi, Q. Wan, Nat. Commun. 2014, 5, 3158.

[444] J. Zhu, Y. Yang, R. Jia, Z. Liang, W. Zhu, Z. U. Rehman, L. Bao, X. Zhang, Y. Cai, L. Song, R. Huang, Adv. Mater. 2018, 30, 1800195.

[445] A. M. Shen, K. Kim, A. Tudor, D. Lee, Y. Chen, Small 2015, 11, 1571.

[446] Y. Yao, X. Huang, S. Peng, D. Zhang, J. Shi, G. Yu, Q. Liu, Z. Jin, Adv. Electron. Mater. 2019, 5, 1800887.

[447] H. Tian, W. Mi, X. F. Wang, H. Zhao, Q. Y. Xie, C. Li, Y. X. Li, Y. Yang, T. L. Ren, Nano Lett. 2015, 15, 8013.

[448] H. Tian, B. Deng, M. L. Chin, X. Yan, H. Jiang, S. J. Han, V. Sun, Q. Xia, M. Dubey, F. Xia, H. Wang, ACS Nano 2016, 10, 10428 .

[449] X. Chen, J. Pan, J. Fu, X. Zhu, C. Zhang, L. Zhou, Y. Wang, Z. Lv, Y. Zhou, S.-T. Han, Adv. Electron. Mater. 2018, 4, 1800444.

[450] Y. Ren, J.-Q. Yang, L. Zhou, J.-Y. Mao, S.-R. Zhang, Y. Zhou, S.-T. Han, Adv. Funct. Mater. 2018, 28, 1805599.

[451] J. Kwon, S. Kyung, S. Yoon, J. J. Kim, S. Jung, Adv. Sci. 2016, 3, 1500439.

[452] H. Seong, J. Choi, B. C. Jang, M. Kim, S. Yoo, S.-Y. Choi, S. G. Im, Adv. Electron. Mater. 2016, 2, 1500385.

[453] H. Yoo, M. Ghittorelli, E. C. Smits, G. H. Gelinck, H. K. Lee, F. Torricelli, J. J. Kim, Sci. Rep. 2016, 6, 35585

[454] S. Fabiano, H. Usta, R. Forchheimer, X. Crispin, A. Facchetti, M. Berggren, Adv. Mater. 2014, 26, 7438

[455] Y. Zhou, S. T. Han, P. Sonar, X. Ma, J. Chen, Z. Zheng, V. A. Roy, Sci. Rep. 2015, 5, 9446.

[456] K. J. Baeg, M. Caironi, Y. Y. Noh, Adv. Mater. 2013, 25, 4210.

[457] H. Yoo, M. Ghittorelli, D. K. Lee, E. C. P. Smits, G. H. Gelinck, H. Ahn, H. K. Lee, F. Torricelli, J. J. Kim, Sci. Rep. 2017, 7, 5015.

[458] J. C. Bijleveld, A. P. Zoombelt, S. G. Mathijssen, M. M. Wienk, M. Turbiez, D. M. de Leeuw, R. A. Janssen, J. Am. Chem. Soc. 2009, $131,16616$.

[459] M. Nakano, I. Osaka, K. Takimiya, Adv. Mater. 2017, 29, 1602893.

[460] M. J. Ford, J. G. Labram, M. Wang, H. Wang, T.-Q. Nguyen, G. C. Bazan, Adv. Electron. Mater. 2017, 3, 1600537.

[461] D. Khim, K.-J. Baeg, M. Caironi, C. Liu, Y. Xu, D.-Y. Kim, Y.-Y. Noh, Adv. Funct. Mater. 2014, 24, 6252.

[462] H. Yoo, E. C. P. Smits, A. J. J. M. van Breemen, J.-L. P. J. van der Steen, F. Torricelli, M. Ghittorelli, J. Lee, G. H. Gelinck, J.--. Kim, Adv. Mater. Technol. 2016, 1, 1600044.

[463] F. Torricelli, M. Ghittorelli, E. C. Smits, C. W. Roelofs, R. A. Janssen, G. H. Gelinck, Z. M. Kovacs-Vajna, E. Cantatore, Adv. Mater. 2016, 28, 284.

[464] H. Yoo, S. B. Lee, D.-K. Lee, E. C. P. Smits, G. H. Gelinck, K. Cho, I.-. Kim, Adv. Electron. Mater. 2018, 4, 1700536.

[465] W. Huang, J. C. Markwart, A. L. Briseno, R. C. Hayward, ACS Nano 2016, 10, 8610

[466] G. Fiori, F. Bonaccorso, G. lannaccone, T. Palacios, D. Neumaier, A. Seabaugh, S. K. Banerjee, L. Colombo, Nat. Nanotechnol. 2014 9, 768.

[467] D. Jariwala, V. K. Sangwan, L. J. Lauhon, T. J. Marks, M. C. Hersam, ACS Nano 2014, 8, 1102.

[468] M. Tosun, S. Chuang, H. Fang, A. B. Sachid, M. Hettick, Y. Lin Y. Zeng, A. Javey, ACS Nano 2014, 8, 4948

[469] Y. Choi, H. Kim, J. Yang, S. W. Shin, S. H. Um, S. Lee, M. S. Kang, J. H. Cho, Chem. Mater. 2018, 30, 4527.

[470] Y.-W. Lan, P.-C. Chen, Y.-Y. Lin, M.-Y. Li, L.-J. Li, Y.-L. Tu, F.-L. Yang, M.-C. Chen, K.-S. Li, Nanoscale Horiz. 2019, 4, 683.

[471] X. Liu, D. Qu, J. Ryu, F. Ahmed, Z. Yang, D. Lee, W. J. Yoo, Adv. Mater. 2016, 28, 2345.

[472] B. Radisavljevic, M. B. Whitwick, A. Kis, Appl. Phys. Lett. 2012, 101 043103

[473] K. K. Kam, B. A. Parkinson, J. Phys. Chem. 1982, 86, 463.
[474] H. S. Lee, S. W. Min, Y. G. Chang, M. K. Park, T. Nam, H. Kim, J. H. Kim, S. Ryu, S. Im, Nano Lett. 2012, 12, 3695.

[475] N. Kaushik, A. Nipane, F. Basheer, S. Dubey, S. Grover M. M. Deshmukh, S. Lodha, Appl. Phys. Lett. 2014, 105, 113505.

[476] C. Kim, I. Moon, D. Lee, M. S. Choi, F. Ahmed, S. Nam, Y. Cho, H. J. Shin, S. Park, W. J. Yoo, ACS Nano 2017, 11, 1588.

[477] S. Chuang, C. Battaglia, A. Azcatl, S. McDonnell, J. S. Kang, X. Yin, M. Tosun, R. Kapadia, H. Fang, R. M. Wallace, A. Javey, Nano Lett. 2014, 14, 1337

[478] A. Nipane, D. Karmakar, N. Kaushik, S. Karande, S. Lodha, ACS Nano 2016, 10, 2128

[479] F. Xue, L. Chen, J. Chen, J. Liu, L. Wang, M. Chen, Y. Pang, X. Yang, G. Gao, J. Zhai, Z. L. Wang, Adv. Mater. 2016, 28, 3391.

[480] B. Radisavljevic, M. B. Whitwick, A. Kis, ACS Nano 2011, 5, 9934.

[481] H. Wang, L. Yu, Y. H. Lee, Y. Shi, A. Hsu, M. L. Chin, L. J. Li, M. Dubey, J. Kong, T. Palacios, Nano Lett. 2012, 12, 4674.

[482] S. Wachter, D. K. Polyushkin, O. Bethge, T. Mueller, Nat. Commun. 2017, 8, 14948

[483] Y. M. Chang, S. H. Yang, C. Y. Lin, C. H. Chen, C. H. Lien, W. B. Jian, K. Ueno, Y. W. Suen, K. Tsukagoshi, Y. F. Lin, Adv. Mater. 2018, 30, 1706995

[484] K. Choi, Y. T. Lee, J. S. Kim, S.-W. Min, Y. Cho, A. Pezeshki, D. K. Hwang, S. Im, Adv. Funct. Mater. 2016, 26, 3146

[485] S. Larentis, B. Fallahazad, H. C. P. Movva, K. Kim, A. Rai, T. Taniguchi, K. Watanabe, S. K. Banerjee, E. Tutuc, ACS Nano 2017, 11, 4832

[486] Y. J. Park, A. K. Katiyar, A. T. Hoang, J. H. Ahn, Small 2019, 15, 1901772.

[487] J. Y. Lim, A. Pezeshki, S. Oh, J. S. Kim, Y. T. Lee, S. Yu, D. K. Hwang, G. H. Lee, H. J. Choi, S. Im, Adv. Mater. 2017, 29, 1701798.

[488] T. Liu, D. Xiang, Y. Zheng, Y. Wang, X. Wang, L. Wang, J. He, L. Liu, W. Chen, Adv. Mater. 2018, 30, 1804470.

[489] W. Zhao, Z. Ghorannevis, L. Chu, M. Toh, C. Kloc, P. H. Tan, G. Eda, ACS Nano 2013, 7, 791.

[490] A. Allain, A. Kis, ACS Nano 2014, 8, 7180

[491] S. Das, M. Dubey, A. Roelofs, Appl. Phys. Lett. 2014, 105, 083511.

[492] J. K. Huang, J. Pu, C. L. Hsu, M. H. Chiu, Z. Y. Juang, Y. H. Chang, W. H. Chang, Y. Iwasa, T. Takenobu, L. J. Li, ACS Nano 2014, 8, 923.

[493] G. V. Resta, Y. Balaji, D. Lin, I. P. Radu, F. Catthoor, P. E. Gaillardon, G. De Micheli, ACS Nano 2018, 12, 7039.

[494] L. Yu, A. Zubair, E. J. Santos, X. Zhang, Y. Lin, Y. Zhang, T. Palacios, Nano Lett. 2015, 15, 4928.

[495] J. Pu, K. Funahashi, C. H. Chen, M. Y. Li, L. J. Li, T. Takenobu, Adv. Mater. 2016, 28, 4111 .

[496] S. P. Koenig, R. A. Doganov, H. Schmidt, A. H. Castro Neto, B. Özyilmaz, Appl. Phys. Lett. 2014, 104, 103106.

[497] L. Chen, S. Li, X. Feng, L. Wang, X. Huang, B. C. K. Tee, K.-W. Ang, Adv. Electron. Mater. 2018, 4, 1800274.

[498] Y. Liu, K. W. Ang, ACS Nano 2017, 11, 7416.

[499] Y. Xu, J. Yuan, K. Zhang, Y. Hou, Q. Sun, Y. Yao, S. Li, Q. Bao, H. Zhang, Y. Zhang, Adv. Funct. Mater. 2017, 27, 1702211.

[500] L. Zhang, L. Y. Shao, G. Gu, T. Wang, X. W. Sun, X. Chen, Adv. Electron. Mater. 2019, 5, 1900133.

[501] Y. Zheng, Z. Hu, C. Han, R. Guo, D. Xiang, B. Lei, Y. Wang, J. He, M. Lai, W. Chen, Nano Res. 2019, 12, 531.

[502] S. P. Koenig, R. A. Doganov, L. Seixas, A. Carvalho, J. Y. Tan, K. Watanabe, T. Taniguchi, N. Yakovlev, A. H. Castro Neto, B. Ozyilmaz, Nano Lett. 2016, 16, 2145

[503] G. Tang, B. Li, J. Lan, J. You, Adv. Mater. Interfaces 2017, 4, 1700453.

[504] K. Maruyama, K. Sawabe, T. Sakanoue, J. Li, W. Takahashi, S. Hotta, Y. Iwasa, T. Takenobu, Sci. Rep. 2015, 5, 10221.

[505] I. Ikezoe, H. Tanaka, K. Hiraoka, H. Kajii, Y. Ohmori, Org. Electron. 2014, 15, 105 
[506] H. Kajii, H. Tanaka, Y. Kusumoto, T. Ohtomo, Y. Ohmori, Org. Electron. 2015, 16, 26.

[507] M. Natali, S. D. Quiroga, L. Passoni, L. Criante, E. Benvenuti, G. Bolognini, L. Favaretto, M. Melucci, M. Muccini, F. Scotognella, F. Di Fonzo, S. Toffanin, Adv. Funct. Mater. 2017, 27, 1605164.

[508] D.-K. Kim, J.-D. Oh, E.-S. Shin, H.-S. Seo, J.-H. Choi, J. Appl. Phys. 2014, 115, 164503.

[509] S. Hotta, T. Yamao, S. Z. Bisri, T. Takenobu, Y. Iwasa, J. Mater. Chem. C 2014, 2, 965.

[510] C. Rost, S. Karg, W. Riess, M. A. Loi, M. Murgia, M. Muccini, Appl. Phys. Lett. 2004, 85, 1613.

[511] C. Zhang, P. Chen, W. Hu, Small 2016, 12, 1252.

[512] C. F. Liu, X. Liu, W. Y. Lai, W. Huang, Adv. Mater. 2018, 30, 1802466.

[513] A. Hepp, H. Heil, W. Weise, M. Ahles, R. Schmechel, H. von Seggern, Phys. Rev. Lett. 2003, 91, 157406.

[514] J. Zaumseil, C. L. Donley, J. S. Kim, R. H. Friend, H. Sirringhaus, Adv. Mater. 2006, 18, 2708.

[515] T. Takenobu, S. Z. Bisri, T. Takahashi, M. Yahiro, C. Adachi, Y. Iwasa, Phys. Rev. Lett. 2008, 100, 066601.

[516] M. A. Baldo, R. J. Holmes, S. R. Forrest, Phys. Rev. B 2002, 66, 035321.

[517] K. Sawabe, T. Takenobu, S. Bisri, T. Yamao, S. Hotta, Y. Iwasa, Appl. Phys. Lett. 2010, 97, 043307.

[518] R. Capelli, S. Toffanin, G. Generali, H. Usta, A. Facchetti, M. Muccini, Nat. Mater. 2010, 9, 496.

[519] M. C. Gwinner, D. Kabra, M. Roberts, T. J. Brenner, B. H. Wallikewitz, C. R. McNeill, R. H. Friend, H. Sirringhaus, Adv. Mater. 2012, 24, 2728.

[520] E. B. Namdas, B. B. Y. Hsu, Z. Liu, S. C. Lo, P. L. Burn, I. D. W. Samuel, Adv. Mater. 2009, 21, 4957.

[521] S. Z. Bisri, T. Takenobu, Y. Iwasa, J. Mater. Chem. C 2014, 2, 2827.

[522] I. D. W. Samuel, E. B. Namdas, G. A. Turnbull, Nat. Photonics 2009, 3, 546.

[523] M. C. Gwinner, S. Khodabakhsh, M. H. Song, H. Schweizer, H. Giessen, H. Sirringhaus, Adv. Funct. Mater. 2009, 19, 1360

[524] F. Sasaki, M. Mori, S. Haraichi, Y. Ido, Y. Masumoto, S. Hotta, Org. Electron. 2010, 11, 1192

[525] H. Wang, F. Li, I. Ravia, B. Gao, Y. Li, V. Medvedev, H. Sun, N. Tessler, Y. Ma, Adv. Funct. Mater. 2011, 21, 3770.

[526] S.-J. Yoon, J. W. Chung, J. Gierschner, K. S. Kim, M.-G. Choi, D. Kim, S. Y. Park, J. Am. Chem. Soc. 2010, 132, 13675.

[527] N. C. Giebink, S. R. Forrest, Phys. Rev. B 2009, 79, 073302.

[528] S. Schols, A. Kadashchuk, P. Heremans, A. Helfer, U. Scherf, ChemPhysChem 2009, 10, 1071.

[529] S. K. Park, J. H. Kim, T. Ohto, R. Yamada, A. O. F. Jones, D. R. Whang, I. Cho, S. Oh, S. H. Hong, J. E. Kwon, J. H. Kim, Y. Olivier, R. Fischer, R. Resel, J. Gierschner, H. Tada, S. Y. Park, Adv. Mater. 2017, 29, 1701346.

[530] B. B. Hsu, C. Duan, E. B. Namdas, A. Gutacker, J. D. Yuen, F. Huang, Y. Cao, G. C. Bazan, I. D. Samuel, A. J. Heeger, Adv. Mater. 2012, 24, 1171.

[531] T. Yamao, Y. Sakurai, K. Terasaki, Y. Shimizu, H. Jinnai, S. Hotta, Adv. Mater. 2010, 22, 3708.

[532] S. P. Kersten, A. J. Schellekens, B. Koopmans, P. A. Bobbert, Phys. Rev. Lett. 2011, 106, 197402.
[533] M. Kemerink, D. S. H. Charrier, E. C. P. Smits, S. G. J. Mathijssen, D. M. d. Leeuw, R. A. J. Janssen, Appl. Phys. Lett. 2008, 93, 033312.

[534] Y. Mashiko, D. Taguchi, M. Weis, T. Manaka, M. Iwamoto, Appl. Phys. Lett. 2012, 101, 243302.

[535] J. Zaumseil, X. Ho, J. R. Guest, G. P. Wiederrecht, J. A. Rogers, ACS Nano 2009, 3, 2225.

[536] E. J. Feldmeier, M. Schidleja, C. Melzer, H. von Seggern, Adv. Mater. 2010, 22, 3568

[537] J. Pu, T. Takenobu, Adv. Mater. 2018, 30, 1707627.

[538] K. F. Mak, J. Shan, Nat. Phys. 2016, 10, 216.

[539] F. Xia, H. Wang, D. Xiao, M. Dubey, A. Ramasubramaniam, Nat. Photonics 2014, 8, 899.

[540] H. C. Movva, A. Rai, S. Kang, K. Kim, B. Fallahazad, T. Taniguchi, K. Watanabe, E. Tutuc, S. K. Banerjee, ACS Nano 2015, 9, 10402.

[541] S. Z. Bisri, S. Shimizu, M. Nakano, Y. Iwasa, Adv. Mater. 2017, 29, 1607054

[542] J. Lee, M. J. Panzer, Y. He, T. P. Lodge, C. D. Frisbie, J. Am. Chem. Soc. 2007, 129, 4532.

[543] K. Ueno, H. Shimotani, H. Yuan, J. Ye, M. Kawasaki, Y. Iwasa, J. Phys. Soc. Jpn. 2014, 83, 032001.

[544] I. Gutiérrez-Lezama, B. A. Reddy, N. Ubrig, A. F. Morpurgo, 2D Mater. 2016, 3, 045016.

[545] D. Kozawa, J. Pu, R. Shimizu, S. Kimura, M.-H. Chiu, K. Matsuki, Y. Wada, T. Sakanoue, Y. Iwasa, L.-J. Li, T. Takenobu, Appl. Phys. Lett. 2016, 109, 201107.

[546] M. Onga, Y. Zhang, R. Suzuki, Y. Iwasa, Appl. Phys. Lett. 2016, 108, 073107.

[547] Y. Zhang, R. Suzuki, Y. Iwasa, ACS Nano 2017, 11, 12583.

[548] Y. J. Zhang, T. Oka, R. Suzuki, J. T. Ye, Y. Iwasa, Science 2014, 344, 725.

[549] J. R. Schaibley, H. Yu, G. Clark, P. Rivera, J. S. Ross, K. L. Seyler, W. Yao, X. Xu, Nat. Rev. Mater. 2016, 1, 16055

[550] D. Xiao, G. B. Liu, W. Feng, X. Xu, W. Yao, Phys. Rev. Lett. 2012, 108, 196802

[551] X. Xu, W. Yao, D. Xiao, T. F. Heinz, Nat. Phys. 2014, 10, 343.

[552] T. Cao, G. Wang, W. Han, H. Ye, C. Zhu, J. Shi, Q. Niu, P. Tan, E. Wang, B. Liu, J. Feng, Nat. Commun. 2012, 3, 887.

[553] A. M. Jones, H. Yu, N. J. Ghimire, S. Wu, G. Aivazian, J. S. Ross, B. Zhao, J. Yan, D. G. Mandrus, D. Xiao, W. Yao, X. Xu, Nat. Nanotechnol. 2013, 8, 634.

[554] K. F. Mak, K. He, C. Lee, G. H. Lee, J. Hone, T. F. Heinz, J. Shan, Nat. Mater. 2013, 12, 207.

[555] K. F. Mak, K. He, J. Shan, T. F. Heinz, Nat. Nanotechnol. 2012, 7, 494.

[556] H. Zeng, J. Dai, W. Yao, D. Xiao, X. Cui, Nat. Nanotechnol. 2012, 7, 490 .

[557] O. L. Sanchez, D. Ovchinnikov, S. Misra, A. Allain, A. Kis, Nano Lett. 2016, 16, 5792.

[558] Y. Ye, J. Xiao, H. Wang, Z. Ye, H. Zhu, M. Zhao, Y. Wang, J. Zhao, X. Yin, X. Zhang, Nat. Nanotechnol. 2016, 11, 598.

[559] W. Yang, J. Shang, J. Wang, X. Shen, B. Cao, N. Peimyoo, C. Zou, Y. Chen, Y. Wang, C. Cong, W. Huang, T. Yu, Nano Lett. 2016, 16, 1560.

[560] H. Yu, Y. Wu, G. B. Liu, X. Xu, W. Yao, Phys. Rev. Lett. 2014, 113, 156603. 